\title{
Synthesis of 1,4-Dihydropyridines and Related Heterocycles by Iodine-Mediated Annulation Reactions of $N$-Cyclopropyl Enamines
}

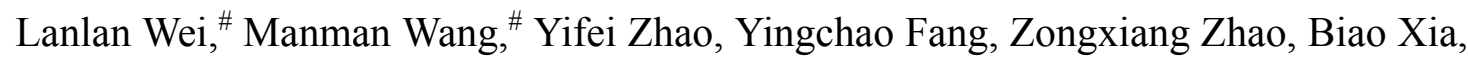
Wenquan $\mathrm{Yu}, *$ and Junbiao Chang*

(" ${ }^{\#}$. Wei and M. Wang contributed equally to this work)

Green Catalysis Center, and College of Chemistry, Zhengzhou University, Zhengzhou, Henan Province 450001, China.

\section{CONTENTS:}

1. General Information S2

2. General Procedure for the Preparation of Substrates 1 S2

3. General Procedure for the Synthesis of 1,4-Dihydropyridines 2

4. Procedure for the Gram-Scale Synthesis of 2a

5. Isolation and Characterization of Intermediate $\mathbf{J}$

6. Capture of Cyclopropylamine Formed during the Synthesis of 2a

7. General Procedure for the Synthesis of Pyridines 3

8. General Procedure for the Synthesis of Compounds $\mathbf{4}$ and $\mathbf{5}$ from $\mathbf{2}$

9. Synthesis of Compound 5a from $4 \mathbf{a}$

10. Synthesis of Compound 5a from $\mathbf{2 a}$

11. X-ray Structures and Data of Compounds 2a, 3d, 4f and 5a S26

12. References 
1. General Information. ${ }^{1} \mathrm{H}$ and ${ }^{13} \mathrm{C}$ NMR spectra were recorded on a $400 \mathrm{MHz}(100$ $\mathrm{MHz}$ for ${ }^{13} \mathrm{C} \mathrm{NMR}$ ) spectrometer. Chemical shift values are given in ppm (parts per million) with tetramethylsilane (TMS) as an internal standard. The peak patterns are reported as follows: s, singlet; $d$, doublet; t, triplet; q, quartet; quint, quintet; sext, sextet; hept, heptet; m, multiplet; dd, doublet of doublets; dt, doublet of triplets. The coupling constants $(J)$ are reported in Hertz $(\mathrm{Hz})$. Melting points were determined on a micromelting point apparatus and are uncorrected. High-resolution mass spectra (HRMS) were obtained on a Q-TOF Mass Spectrometer equipped with an electrospray ion source (ESI), and operated in the positive mode. Flash column chromatography was performed over silica gel 200-300 mesh. 1,2-Dichoroethane (DCE) used for the synthesis of products 2 was analytical reagent grade and used without any pretreatment.

2. General Procedure for the Preparation of Substrates 1. A reaction mixture containing a substituted 1,3-dicarbonyl compound (10 mmol), cyclopropylamine (3.5 $\mathrm{mL}, 50 \mathrm{mmol})$ and acetic acid $(2.9 \mathrm{~mL}, 50 \mathrm{mmol})$ in EtOH $(20 \mathrm{~mL})$ was heated to reflux with a heating mantle until TLC indicated that consumption of 1,3-dicarbonyl compound was complete. After cooling to room temperature, the mixture was neutralized with aqueous $\mathrm{NaHCO}_{3}$, and then extracted with EtOAc $(20 \mathrm{~mL} \times 3)$. The combined organic layer was dried over anhydrous $\mathrm{Na}_{2} \mathrm{SO}_{4}$, then concentrated and purified by silica gel column chromatography to give the enamine substrate (1).

Methyl (Z)-3-(cyclopropylamino)-3-phenylacrylate (1a). Eluent: EtOAc/petroleum ether (PE) 5:95; 3 h; yield: 2.06 g, 95\%; colorless oil; ${ }^{1} \mathrm{H}$ NMR (400 MHz, $\left.\mathrm{CDCl}_{3}\right) \delta$ $8.60(\mathrm{~s}, 1 \mathrm{H}), 7.46-7.43(\mathrm{~m}, 2 \mathrm{H}), 7.41-7.38(\mathrm{~m}, 3 \mathrm{H}), 4.62(\mathrm{~s}, 1 \mathrm{H}), 3.67(\mathrm{~s}, 3 \mathrm{H})$, 2.53-2.47 (m, 1H), 0.56-0.47 (m, 4H); ${ }^{13} \mathrm{C} \mathrm{NMR}\left(100 \mathrm{MHz}, \mathrm{CDCl}_{3}\right) \delta$ 170.7, 165.5, 
136.8, 129.0, 128.1, 127.9, 84.8, 50.2, 26.8, 8.6; HRMS (ESI-TOF) m/z: $[\mathrm{M}+\mathrm{H}]^{+}$ calcd for $\mathrm{C}_{13} \mathrm{H}_{16} \mathrm{NO}_{2} 218.1176$, found 218.1174.

Methyl (Z)-3-(cyclopropylamino)-3-(p-tolyl)acrylate (1b). Eluent: EtOAc/PE 5:95; 2 h; yield: $2.01 \mathrm{~g}, 87 \%$; colorless oil; ${ }^{1} \mathrm{H}$ NMR (400 MHz, $\left.\mathrm{CDCl}_{3}\right) \delta 8.59$ (s, 1H), 7.35 $(\mathrm{d}, J=8.0 \mathrm{~Hz}, 2 \mathrm{H}), 7.19(\mathrm{~d}, J=7.6 \mathrm{~Hz}, 2 \mathrm{H}), 4.61(\mathrm{~s}, 1 \mathrm{H}), 3.66(\mathrm{~s}, 3 \mathrm{H}), 2.54-2.47(\mathrm{~m}$, 1H), $2.38(\mathrm{~s}, 3 \mathrm{H}), 0.57-0.48(\mathrm{~m}, 4 \mathrm{H}) ;{ }^{13} \mathrm{C} \mathrm{NMR}\left(100 \mathrm{MHz}, \mathrm{CDCl}_{3}\right) \delta$ 170.8, 165.7, 139.1, 133.9, 128.8, 127.9, 84.5, 50.2, 26.9, 21.3, 8.7; HRMS (ESI-TOF) m/z: [M + $\mathrm{H}]^{+}$calcd for $\mathrm{C}_{14} \mathrm{H}_{18} \mathrm{NO}_{2} 232.1332$, found 232.1333.

Methyl (Z)-3-(cyclopropylamino)-3-(4-methoxyphenyl)acrylate (1c). Eluent: EtOAc/PE 2:98; 3 h; yield: 1.93 g, 78\%; colorless oil; ${ }^{1} \mathrm{H}$ NMR (400 MHz, $\left.\mathrm{CDCl}_{3}\right) \delta$ $8.60(\mathrm{~s}, 1 \mathrm{H}), 7.42(\mathrm{dt}, J=9.6,2.8 \mathrm{~Hz}, 2 \mathrm{H}), 6.91(\mathrm{dt}, J=9.6,2.8 \mathrm{~Hz}, 2 \mathrm{H}), 4.61(\mathrm{~s}, 1 \mathrm{H})$, $3.84(\mathrm{~s}, 3 \mathrm{H}), 3.66(\mathrm{~s}, 3 \mathrm{H}), 2.56-2.49(\mathrm{~m}, 1 \mathrm{H}), 0.59-0.54(\mathrm{~m}, 2 \mathrm{H}), 0.53-0.49(\mathrm{~m}, 2 \mathrm{H})$; ${ }^{13} \mathrm{C}$ NMR $\left(100 \mathrm{MHz}, \mathrm{CDCl}_{3}\right) \delta 170.8,165.3,160.3,129.4,129.0,113.5,84.4,55.3$, 50.2, 27.0, 8.7; HRMS (ESI-TOF) m/z: $[\mathrm{M}+\mathrm{H}]^{+}$calcd for $\mathrm{C}_{14} \mathrm{H}_{18} \mathrm{NO}_{3} 248.1281$, found 248.1284 .

Methyl (Z)-3-(cyclopropylamino)-3-(4-fluorophenyl)acrylate (1d). Eluent: EtOAc/PE 2:98; 3 h; yield: 2.00 g, 85\%; colorless oil; ${ }^{1} \mathrm{H}$ NMR (400 MHz, $\left.\mathrm{CDCl}_{3}\right) \delta$ $8.58(\mathrm{~s}, 1 \mathrm{H}), 7.47-7.42(\mathrm{~m}, 2 \mathrm{H}), 7.12-7.06(\mathrm{~m}, 2 \mathrm{H}), 4.60(\mathrm{~s}, 1 \mathrm{H}), 3.66(\mathrm{~s}, 3 \mathrm{H})$, 2.50-2.44 (m, 1H), 0.58-0.53 (m, 2H), 0.51-0.47 (m, 2H); ${ }^{13} \mathrm{C}$ NMR (100 MHz, $\left.\mathrm{CDCl}_{3}\right) \delta 170.6,164.3\left(\mathrm{~d}, J_{\mathrm{C}-\mathrm{F}}=2.1 \mathrm{~Hz}\right), 161.9,132.8\left(\mathrm{~d}, J_{\mathrm{C}-\mathrm{F}}=3.4 \mathrm{~Hz}\right), 129.8(\mathrm{~d}$, $\left.J_{\mathrm{C}-\mathrm{F}}=8.3 \mathrm{~Hz}\right), 115.2\left(\mathrm{~d}, J_{\mathrm{C}-\mathrm{F}}=21.6 \mathrm{~Hz}\right), 85.1,50.3,26.8,8.7 ;{ }^{19} \mathrm{~F}$ NMR $(376 \mathrm{MHz}$, $\left.\mathrm{CDCl}_{3}\right) \delta$-111.9; HRMS (ESI-TOF) $\mathrm{m} / \mathrm{z}:[\mathrm{M}+\mathrm{Na}]^{+}$calcd for $\mathrm{C}_{13} \mathrm{H}_{14} \mathrm{FNNaO}_{2}$ 258.0901, found 258.0903.

Methyl (Z)-3-(4-chlorophenyl)-3-(cyclopropylamino)acrylate (1e). Eluent: EtOAc/PE 5:95; 3 h; yield: 2.09 g, 83\%; white solid, mp $36-38{ }^{\circ} \mathrm{C} ;{ }^{1} \mathrm{H}$ NMR (400 
$\left.\mathrm{MHz}, \mathrm{CDCl}_{3}\right) \delta 8.55(\mathrm{~s}, 1 \mathrm{H}), 7.41-7.36(\mathrm{~m}, 4 \mathrm{H}), 4.60(\mathrm{~s}, 1 \mathrm{H}), 3.66(\mathrm{~s}, 3 \mathrm{H}), 2.49-2.43$ $(\mathrm{m}, 1 \mathrm{H}), 0.57-0.47(\mathrm{~m}, 4 \mathrm{H}) ;{ }^{13} \mathrm{C}$ NMR $\left(100 \mathrm{MHz}, \mathrm{CDCl}_{3}\right) \delta 170.6,164.1,135.2$, 135.1, 129.3, 128.4 , 85.2, 50.3, 26.8, 8.8; HRMS (ESI-TOF) m/z: $[\mathrm{M}+\mathrm{H}]^{+}$calcd for $\mathrm{C}_{13} \mathrm{H}_{15} \mathrm{ClNO}_{2} 252.0786$, found 252.0787.

Methyl (Z)-3-(4-bromophenyl)-3-(cyclopropylamino)acrylate (1f). Eluent: EtOAc/PE 5:95; 3 h; yield: 2.40 g, 81\%; colorless oil; ${ }^{1} \mathrm{H}$ NMR (400 MHz, $\left.\mathrm{CDCl}_{3}\right) \delta$ 8.55 (s, 1H), 7.54 (dt, $J=8.8,2.4 \mathrm{~Hz}, 2 \mathrm{H}), 7.33(\mathrm{dt}, J=8.8,2.4 \mathrm{~Hz}, 2 \mathrm{H}), 4.60(\mathrm{~s}, 1 \mathrm{H})$, $3.66(\mathrm{~s}, 3 \mathrm{H}), 2.49-2.43(\mathrm{~m}, 1 \mathrm{H}), 0.58-0.53(\mathrm{~m}, 2 \mathrm{H}), 0.51-0.47(\mathrm{~m}, 2 \mathrm{H}) ;{ }^{13} \mathrm{C} \mathrm{NMR}$ $\left(100 \mathrm{MHz}, \mathrm{CDCl}_{3}\right) \delta 170.6,164.1,135.7,131.4,129.6,123.4,85.2,50.4,26.8,8.8$ HRMS (ESI-TOF) m/z: [M + H] $]^{+}$calcd for $\mathrm{C}_{13} \mathrm{H}_{15} \mathrm{BrNO}_{2} 296.0281$, found 296.0284.

Methyl (Z)-3-(cyclopropylamino)-3-(4-(trifluoromethyl)phenyl)acrylate (1g). Eluent: EtOAc/PE 6:94; 2 h; yield: 2.74 g, 96\%; yellow oil; ${ }^{1} \mathrm{H}$ NMR (400 MHz, $\left.\mathrm{CDCl}_{3}\right) \delta 8.57(\mathrm{~s}, 1 \mathrm{H}), 7.67(\mathrm{~d}, J=8.4 \mathrm{~Hz}, 2 \mathrm{H}), 7.57(\mathrm{~d}, J=8.4 \mathrm{~Hz}, 2 \mathrm{H}), 4.62(\mathrm{~s}, 1 \mathrm{H})$, $3.68(\mathrm{~s}, 3 \mathrm{H}), 2.48-2.42(\mathrm{~m}, 1 \mathrm{H}), 0.57-0.52(\mathrm{~m}, 2 \mathrm{H}), 0.51-0.47(\mathrm{~m}, 2 \mathrm{H}) ;{ }^{13} \mathrm{C} \mathrm{NMR}$ $\left(100 \mathrm{MHz}, \mathrm{CDCl}_{3}\right) \delta 170.5,163.7,140.4,131.1\left(\mathrm{q}, J_{\mathrm{C}-\mathrm{F}}=32.5 \mathrm{~Hz}\right), 128.4,125.2(\mathrm{q}$, $\left.J_{\mathrm{C}-\mathrm{F}}=3.8 \mathrm{~Hz}\right), 123.9\left(\mathrm{q}, J_{\mathrm{C}-\mathrm{F}}=270.6 \mathrm{~Hz}\right), 85.7,50.4,26.8,8.8 ;{ }^{19} \mathrm{~F} \mathrm{NMR}(376 \mathrm{MHz}$, $\left.\mathrm{CDCl}_{3}\right) \delta$-62.7; HRMS (ESI-TOF) m/z: $[\mathrm{M}+\mathrm{H}]^{+}$calcd for $\mathrm{C}_{14} \mathrm{H}_{15} \mathrm{~F}_{3} \mathrm{NO}_{2} 286.1049$, found 286.1051 .

Methyl (Z)-3-(4-cyanophenyl)-3-(cyclopropylamino)acrylate (1h). Eluent: EtOAc/PE 5:95; 5 h; yield: 2.11 g, 87\%; white solid, mp 84-86 ${ }^{\circ} \mathrm{C}$; ${ }^{1} \mathrm{H}$ NMR (400 $\left.\mathrm{MHz}, \mathrm{CDCl}_{3}\right) \delta 8.54(\mathrm{~s}, 1 \mathrm{H}), 7.71(\mathrm{dt}, J=8.8,2.0 \mathrm{~Hz}, 2 \mathrm{H}), 7.57(\mathrm{dt}, J=8.4,2.0 \mathrm{~Hz}$, $2 \mathrm{H}), 4.61(\mathrm{~s}, 1 \mathrm{H}), 3.68(\mathrm{~s}, 3 \mathrm{H}), 2.47-2.41(\mathrm{~m}, 1 \mathrm{H}), 0.57-0.52(\mathrm{~m}, 2 \mathrm{H}), 0.51-0.46(\mathrm{~m}$, $2 \mathrm{H}) ;{ }^{13} \mathrm{C} \mathrm{NMR}\left(100 \mathrm{MHz}, \mathrm{CDCl}_{3}\right) \delta 170.3,162.9,141.3,132.0,128.7,118.3,112.9$, 86.1, 50.5, 26.7, 8.8; HRMS (ESI-TOF) m/z: $[\mathrm{M}+\mathrm{H}]^{+}$calcd for $\mathrm{C}_{14} \mathrm{H}_{15} \mathrm{~N}_{2} \mathrm{O}_{2}$ 243.1128 , found 243.1131 . 
Methyl (Z)-3-(cyclopropylamino)-3-(m-tolyl)acrylate (1i). Eluent: EtOAc/PE 5:95; 1.5 h; yield: 2.06 g, 89\%; light yellow oil; ${ }^{1} \mathrm{H} \mathrm{NMR}\left(400 \mathrm{MHz}, \mathrm{CDCl}_{3}\right) \delta 8.58(\mathrm{~s}, 1 \mathrm{H})$, 7.30-7.23 (m, 3H, overlapped with the peak of chloroform), 7.21-7.20 (m, 1H), 4.61 (s, 1H), $3.66(\mathrm{~s}, 3 \mathrm{H}), 2.54-2.47(\mathrm{~m}, 1 \mathrm{H}), 2.38(\mathrm{~s}, 3 \mathrm{H}), 0.56-0.48(\mathrm{~m}, 4 \mathrm{H}) ;{ }^{13} \mathrm{C} \mathrm{NMR}$ $\left(100 \mathrm{MHz}, \mathrm{CDCl}_{3}\right) \delta 170.8,165.7,137.8,136.7,129.8,128.5,128.0,125.0,84.6,50.2$ 26.9, 21.4, 8.6; HRMS (ESI-TOF) m/z: $[\mathrm{M}+\mathrm{H}]^{+}$calcd for $\mathrm{C}_{14} \mathrm{H}_{18} \mathrm{NO}_{2} 232.1332$, found 232.1334 .

Methyl (Z)-3-(cyclopropylamino)-3-(2-fluorophenyl)acrylate (1j). Eluent: EtOAc/PE 1:99; 2 h; yield: 2.14 g, 91\%; colorless oil; ${ }^{1} \mathrm{H}$ NMR (400 MHz, $\left.\mathrm{CDCl}_{3}\right) \delta$ $8.61(\mathrm{~s}, 1 \mathrm{H}), 7.42-7.36(\mathrm{~m}, 1 \mathrm{H}), 7.29(\mathrm{td}, J=7.6,2.0 \mathrm{~Hz}, 1 \mathrm{H}$, overlapped with the peak of chloroform), $7.18(\mathrm{td}, J=7.6,0.8 \mathrm{~Hz}, 1 \mathrm{H}), 7.13-7.09(\mathrm{~m}, 1 \mathrm{H}), 4.59(\mathrm{~s}, 1 \mathrm{H})$, $3.67(\mathrm{~s}, 3 \mathrm{H}), 2.50-2.47(\mathrm{~m}, 1 \mathrm{H}), 0.45-0.43(\mathrm{~m}, 4 \mathrm{H}) ;{ }^{13} \mathrm{C} \mathrm{NMR}\left(100 \mathrm{MHz}, \mathrm{CDCl}_{3}\right) \delta$ $170.5,160.0,159.0\left(\mathrm{~d}, J_{\mathrm{C}-\mathrm{F}}=247.1 \mathrm{~Hz}\right), 130.8\left(\mathrm{~d}, J_{\mathrm{C}-\mathrm{F}}=8.0 \mathrm{~Hz}\right), 130.0\left(\mathrm{~d}, J_{\mathrm{C}-\mathrm{F}}=3.2\right.$ $\mathrm{Hz}), 124.8\left(\mathrm{~d}, J_{\mathrm{C}-\mathrm{F}}=16.1 \mathrm{~Hz}\right), 124.1\left(\mathrm{~d}, J_{\mathrm{C}-\mathrm{F}}=3.5 \mathrm{~Hz}\right), 115.6\left(\mathrm{~d}, J_{\mathrm{C}-\mathrm{F}}=21.6 \mathrm{~Hz}\right)$, $85.3,50.3,25.7\left(\mathrm{~d}, J_{\mathrm{C}-\mathrm{F}}=3.4 \mathrm{~Hz}\right), 7.7 ;{ }^{19} \mathrm{~F} \mathrm{NMR}\left(376 \mathrm{MHz}, \mathrm{CDCl}_{3}\right) \delta$-113.4; HRMS (ESI-TOF) m/z: [M + H $]^{+}$calcd for $\mathrm{C}_{13} \mathrm{H}_{15} \mathrm{FNO}_{2} 236.1081$, found 236.1088.

Methyl (Z)-3-(cyclopropylamino)-3-(3,4-dimethylphenyl)acrylate (1k). Eluent: EtOAc/PE 20:80; 3 h; yield: 2.16 g, 88\%; colorless oil; ${ }^{1} \mathrm{H}$ NMR (400 MHz, $\left.\mathrm{CDCl}_{3}\right) \delta$ $8.58(\mathrm{~s}, 1 \mathrm{H}), 7.23-7.19(\mathrm{~m}, 2 \mathrm{H}), 7.15(\mathrm{~d}, J=7.6 \mathrm{~Hz}, 1 \mathrm{H}), 4.61(\mathrm{~s}, 1 \mathrm{H}), 3.66(\mathrm{~s}, 3 \mathrm{H})$, $2.56-2.49(\mathrm{~m}, 1 \mathrm{H}), 2.29(\mathrm{~s}, 6 \mathrm{H}), 0.56-0.51(\mathrm{~m}, 4 \mathrm{H}) ;{ }^{13} \mathrm{C} \mathrm{NMR}\left(100 \mathrm{MHz}, \mathrm{CDCl}_{3}\right) \delta$ $170.8,165.8,137.8,136.4,134.2,129.3,129.1,125.4,84.4,50.2,26.9,19.8,19.7,8.6$; HRMS (ESI-TOF) m/z: [M + H] $]^{+}$calcd for $\mathrm{C}_{15} \mathrm{H}_{20} \mathrm{NO}_{2} 246.1489$, found 246.1493 .

Methyl (Z)-3-(benzo $[d][1,3]$ dioxol-5-yl)-3-(cyclopropylamino)acrylate (11). Eluent: EtOAc/PE 20:80; 3 h; yield: 2.33 g, 89\%; colorless oil; ${ }^{1} \mathrm{H}$ NMR (400 MHz, $\left.\mathrm{CDCl}_{3}\right) \delta$ $8.55(\mathrm{~s}, 1 \mathrm{H}), 6.97-6.95(\mathrm{~m}, 2 \mathrm{H}), 6.83(\mathrm{~d}, J=8.0 \mathrm{~Hz}, 1 \mathrm{H}), 6.01(\mathrm{~d}, J=0.4 \mathrm{~Hz}, 2 \mathrm{H})$, 
$4.61(\mathrm{~s}, 1 \mathrm{H}), 3.66(\mathrm{~s}, 3 \mathrm{H}), 2.56-2.49(\mathrm{~m}, 1 \mathrm{H}), 0.60-0.56(\mathrm{~m}, 2 \mathrm{H}), 0.54-0.49(\mathrm{~m}, 2 \mathrm{H})$;

${ }^{13} \mathrm{C} \mathrm{NMR}\left(100 \mathrm{MHz}, \mathrm{CDCl}_{3}\right) \delta 170.7,165.0,148.3,147.3,130.5,122.0,108.5,108.1$, 101.3, 84.6, 50.3, 26.9, 8.7; HRMS (ESI-TOF) m/z: $[\mathrm{M}+\mathrm{H}]^{+}$calcd for $\mathrm{C}_{14} \mathrm{H}_{16} \mathrm{NO}_{4}$ 262.1074, found 262.1079.

Methyl (Z)-3-(cyclopropylamino)-3-(naphthalen-2-yl)acrylate (1m). Eluent: EtOAc/PE 5:95; 3 h; yield: 2.25 g, 84\%; yellow oil; ${ }^{1} \mathrm{H}$ NMR (400 $\left.\mathrm{MHz}, \mathrm{CDCl}_{3}\right) \delta$ $8.66(\mathrm{~s}, 1 \mathrm{H}), 7.94(\mathrm{~d}, J=1.2 \mathrm{~Hz}, 1 \mathrm{H}), 7.88-7.85(\mathrm{~m}, 3 \mathrm{H}), 7.57(\mathrm{dd}, J=8.4,1.6 \mathrm{~Hz}$, 1H), 7.55-7.50 (m, 2H), $4.74(\mathrm{~s}, 1 \mathrm{H}), 3.69(\mathrm{~s}, 3 \mathrm{H}), 2.59-2.52(\mathrm{~m}, 1 \mathrm{H}), 0.56-0.48(\mathrm{~m}$, $4 \mathrm{H}) ;{ }^{13} \mathrm{C} \mathrm{NMR}\left(100 \mathrm{MHz}, \mathrm{CDCl}_{3}\right) \delta 170.7,165.4,134.3,133.4,132.8,128.3,127.71$, 127.67, 127.4, 126.8, 126.5, 125.5, 85.3, 50.3, 27.0, 8.7; HRMS (ESI-TOF) m/z: [M + $\mathrm{H}]^{+}$calcd for $\mathrm{C}_{17} \mathrm{H}_{18} \mathrm{NO}_{2} 268.1332$, found 268.1333.

Methyl (Z)-3-(cyclopropylamino)-3-(thiophen-2-yl)acrylate (1n). Eluent: EtOAc/PE 5:95; 5 h; yield: $1.81 \mathrm{~g}, 81 \%$; yellow oil; ${ }^{1} \mathrm{H}$ NMR (400 MHz, $\left.\mathrm{CDCl}_{3}\right) \delta$ $8.73(\mathrm{~s}, 1 \mathrm{H}), 7.41(\mathrm{ddd}, J=8.0,3.6,1.2 \mathrm{~Hz}, 2 \mathrm{H}), 7.07(\mathrm{dd}, J=5.2,3.6 \mathrm{~Hz}, 1 \mathrm{H}), 4.88$ (s, 1H), $3.66(\mathrm{~s}, 3 \mathrm{H}), 2.75-2.69(\mathrm{~m}, 1 \mathrm{H}), 0.73-0.67(\mathrm{~m}, 2 \mathrm{H}), 0.64-0.60(\mathrm{~m}, 2 \mathrm{H}) ;{ }^{13} \mathrm{C}$ NMR (100 MHz, $\left.\mathrm{CDCl}_{3}\right) \delta 170.6,157.6,137.2,128.8,127.4,127.1,84.9,50.3,27.0$, 9.2; HRMS (ESI-TOF) m/z: $[\mathrm{M}+\mathrm{H}]^{+}$calcd for $\mathrm{C}_{11} \mathrm{H}_{14} \mathrm{NO}_{2} \mathrm{~S}$ 224.0740, found 224.0743 .

Benzyl (Z)-3-(cyclopropylamino)-3-phenylacrylate (10). Eluent: EtOAc/PE 5:95; 2 h; yield: $2.67 \mathrm{~g}, 91 \%$; colorless oil; ${ }^{1} \mathrm{H}$ NMR (400 MHz, $\left.\mathrm{CDCl}_{3}\right) \delta 8.61(\mathrm{~s}, 1 \mathrm{H})$, 7.47-7.43 (m, 2H), 7.40-7.33 (m, 7H), 7.31-7.27 (m, 1H), $5.13(\mathrm{~s}, 2 \mathrm{H}), 4.69(\mathrm{~s}, 1 \mathrm{H})$, 2.53-2.47 (m, 1H), 0.54-0.50 (m, 4H); ${ }^{13} \mathrm{C} \mathrm{NMR}\left(100 \mathrm{MHz}, \mathrm{CDCl}_{3}\right) \delta$ 170.0, 165.7, $137.2,136.7,129.1,128.4,128.1,127.9,127.8,127.7,84.9,64.6,26.9,8.7$; HRMS (ESI-TOF) m/z: [M + H] $]^{+}$calcd for $\mathrm{C}_{19} \mathrm{H}_{20} \mathrm{NO}_{2} 294.1489$, found 294.1492. 
4-Methoxybenzyl (Z)-3-(cyclopropylamino)-3-phenylacrylate (1p). Eluent:

EtOAc/PE 20:80; 2 h; yield: 3.01 g, 93\%; white solid, mp $68-70{ }^{\circ} \mathrm{C} ;{ }^{1} \mathrm{H}$ NMR (400 $\left.\mathrm{MHz}, \mathrm{CDCl}_{3}\right) \delta 8.60(\mathrm{~s}, 1 \mathrm{H}), 7.45-7.42(\mathrm{~m}, 2 \mathrm{H}), 7.40-7.36(\mathrm{~m}, 3 \mathrm{H}), 7.32(\mathrm{~d}, J=8.8$ $\mathrm{Hz}, 2 \mathrm{H}), 6.88(\mathrm{dt}, J=9.6,2.8 \mathrm{~Hz}, 2 \mathrm{H}), 5.06(\mathrm{~s}, 2 \mathrm{H}), 4.66(\mathrm{~s}, 1 \mathrm{H}), 3.80(\mathrm{~s}, 3 \mathrm{H})$, 2.53-2.46 (m, 1H), 0.54-0.49 (m, 4H); ${ }^{13} \mathrm{C} \mathrm{NMR}\left(100 \mathrm{MHz}, \mathrm{CDCl}_{3}\right) \delta$ 170.1, 165.6, $159.3,136.7,129.7,129.3,129.0,128.1,127.9,113.8,85.0,64.4,55.3,26.9,8.7$; HRMS (ESI-TOF) m/z: [M + H] $]^{+}$calcd for $\mathrm{C}_{20} \mathrm{H}_{22} \mathrm{NO}_{3} 324.1594$, found 324.1596.

4-Chlorobenzyl (Z)-3-(cyclopropylamino)-3-phenylacrylate (1q). Eluent: EtOAc/PE 20:80; 2 h; yield: 2.89 g, 88\%; colorless oil; ${ }^{1} \mathrm{H}$ NMR (400 MHz, $\left.\mathrm{CDCl}_{3}\right) \delta$ $8.60(\mathrm{~s}, 1 \mathrm{H}), 7.46-7.44(\mathrm{~m}, 2 \mathrm{H}), 7.40-7.37(\mathrm{~m}, 3 \mathrm{H}), 7.31(\mathrm{~s}, 4 \mathrm{H}), 5.09(\mathrm{~s}, 2 \mathrm{H}), 4.67(\mathrm{~s}$, 1H), 2.53-2.47 (m, 1H), 0.56-0.47 (m, 4H); $\left.{ }^{13} \mathrm{C} \mathrm{NMR} \mathrm{(100} \mathrm{MHz,} \mathrm{CDCl}_{3}\right) \delta 169.8$, $165.9,136.6,135.8,133.5,129.2,129.1,128.6,128.1,127.9,84.6,63.7,26.9,8.7$; HRMS (ESI-TOF) m/z: [M + H] $]^{+}$calcd for $\mathrm{C}_{19} \mathrm{H}_{19} \mathrm{ClNO}_{2} 328.1099$, found 328.1100.

4-Bromobenzyl (Z)-3-(cyclopropylamino)-3-phenylacrylate (1r). Eluent: EtOAc/PE 20:80; 2 h; yield: 2.78 g, 75\%; colorless oil; ${ }^{1} \mathrm{H}$ NMR (400 MHz, $\left.\mathrm{CDCl}_{3}\right) \delta$ $8.59(\mathrm{~s}, 1 \mathrm{H}), 7.48-7.44(\mathrm{~m}, 4 \mathrm{H}), 7.41-7.37$ (m, 3H), 7.26-7.24 (m, 2H, overlapped with the peak of chloroform), $5.07(\mathrm{~s}, 2 \mathrm{H}), 4.67(\mathrm{~s}, 1 \mathrm{H}), 2.53-2.47(\mathrm{~m}, 1 \mathrm{H})$, 0.54-0.49 (m, 4H); ${ }^{13} \mathrm{C}$ NMR (100 MHz, $\left.\mathrm{CDCl}_{3}\right) \delta 169.8,166.0,136.6,136.3,131.5$, 129.5, 129.1, 128.1, 127.9, 121.6, 84.5, 63.7, 26.9, 8.7; HRMS (ESI-TOF) m/z: [M + $\mathrm{H}]^{+}$calcd for $\mathrm{C}_{19} \mathrm{H}_{19} \mathrm{BrNO}_{2} 372.0594$, found 372.0594 .

Ethyl (Z)-3-(cyclopropylamino)-3-phenylacrylate (1s). ${ }^{1}$ Eluent: EtOAc/PE 6:94; 2 h; yield: 2.06 g, 89\%; yellow oil; ${ }^{1} \mathrm{H}$ NMR (400 $\left.\mathrm{MHz} \mathrm{CDCl}_{3}\right) \delta 8.61(\mathrm{~s}, 1 \mathrm{H})$, $7.47-7.43(\mathrm{~m}, 2 \mathrm{H}), 7.41-7.37(\mathrm{~m}, 3 \mathrm{H}), 4.62(\mathrm{~s}, 1 \mathrm{H}), 4.13(\mathrm{q}, J=7.2 \mathrm{~Hz}, 2 \mathrm{H})$, 2.53-2.46 (m, 1H), $1.27(\mathrm{t}, J=7.2 \mathrm{~Hz}, 3 \mathrm{H}), 0.56-0.47(\mathrm{~m}, 4 \mathrm{H}) ;{ }^{13} \mathrm{C}$ NMR (100 MHz, 
$\left.\mathrm{CDCl}_{3}\right) \delta 170.5,165.4,136.8,129.0,128.1,127.9,85.2,58.7,26.8,14.6,8.7$; HRMS

(ESI-TOF) $\mathrm{m} / \mathrm{z}:[\mathrm{M}+\mathrm{H}]^{+}$calcd for $\mathrm{C}_{14} \mathrm{H}_{18} \mathrm{NO}_{2} 232.1332$, found 232.1333.

Hexyl (Z)-3-(cyclopropylamino)-3-phenylacrylate (1t). Eluent: EtOAc/PE 5:95; 2 h; yield: $2.61 \mathrm{~g}, 91 \%$; colorless oil; ${ }^{1} \mathrm{H}$ NMR (400 $\left.\mathrm{MHz}, \mathrm{CDCl}_{3}\right) \delta 8.60(\mathrm{~s}, 1 \mathrm{H})$, 7.47-7.45 (m, 2H), 7.41-7.38 (m, 3H), $4.62(\mathrm{~s}, 1 \mathrm{H}), 4.06(\mathrm{t}, J=6.8 \mathrm{~Hz}, 2 \mathrm{H})$, 2.53-2.46 (m, 1H), 1.67-1.60 (m, 2H), 1.38-1.28 (m, 6H), $0.88(\mathrm{t}, J=7.2 \mathrm{~Hz}, 3 \mathrm{H})$, 0.53-0.49 (m, 4H); ${ }^{13} \mathrm{C}$ NMR (100 MHz, $\left.\mathrm{CDCl}_{3}\right) \delta 170.6,165.3,136.8,129.0,128.1$, 127.9, 85.3, 63.0, 31.5, 29.0, 26.8, 25.7, 22.6, 14.0, 8.6; HRMS (ESI-TOF) m/z: [M + $\mathrm{H}^{+}$calcd for $\mathrm{C}_{18} \mathrm{H}_{26} \mathrm{NO}_{2} 288.1958$, found 288.1962 .

Isopropyl (Z)-3-(cyclopropylamino)-3-phenylacrylate (1u). Eluent: EtOAc/PE 6:94; $2 \mathrm{~h}$; yield: $2.11 \mathrm{~g}, 86 \%$; yellow oil; ${ }^{1} \mathrm{H}$ NMR (400 MHz, $\left.\mathrm{CDCl}_{3}\right) \delta 8.61(\mathrm{~s}, 1 \mathrm{H})$, 7.47-7.43 (m, 2H), 7.40-7.36 (m, 3H), 5.02 (hept, $J=6.4 \mathrm{~Hz}, 1 \mathrm{H}), 4.59(\mathrm{~s}, 1 \mathrm{H})$, 2.52-2.46 (m, 1H), $1.25(\mathrm{~d}, J=6.0 \mathrm{~Hz}, 6 \mathrm{H}), 0.55-0.47(\mathrm{~m}, 4 \mathrm{H}) ;{ }^{13} \mathrm{C} \mathrm{NMR}(100 \mathrm{MHz}$, $\left.\mathrm{CDCl}_{3}\right) \delta 170.1,165.2,136.9,128.9,128.1,127.9,85.8,65.6,26.8,22.2,8.6$; HRMS (ESI-TOF) $\mathrm{m} / \mathrm{z}:[\mathrm{M}+\mathrm{H}]^{+}$calcd for $\mathrm{C}_{15} \mathrm{H}_{20} \mathrm{NO}_{2} 246.1489$, found 246.1488.

Cyclopentyl (Z)-3-(cyclopropylamino)-3-phenylacrylate (1v). Eluent: EtOAc/PE 5:95; 2 h; yield: $2.28 \mathrm{~g}, 84 \%$; colorless oil; ${ }^{1} \mathrm{H}$ NMR (400 MHz, $\left.\mathrm{CDCl}_{3}\right) \delta 8.61(\mathrm{~s}, 1 \mathrm{H})$, 7.46-7.43 (m, 2H), 7.40-7.36 (m, 3H), 5.18-5.14 (m, 1H), 4.59 (s, 1H), 2.52-2.45 (m, 1H), 1.91-1.82 (m, 2H), 1.76-1.67 (m, 4H), 1.61-1.54 (m, 2H), 0.53-0.49 (m, $4 \mathrm{H}) ;{ }^{13} \mathrm{C} \mathrm{NMR}\left(100 \mathrm{MHz}, \mathrm{CDCl}_{3}\right) \delta 170.4,165.1,136.9,128.9,128.0,127.9,85.8$, 75.2, 32.9, 26.8, 23.8, 8.6; HRMS (ESI-TOF) m/z: $[\mathrm{M}+\mathrm{H}]^{+}$calcd for $\mathrm{C}_{17} \mathrm{H}_{22} \mathrm{NO}_{2}$ 272.1645, found 272.1647 .

Cyclohexyl (Z)-3-(cyclopropylamino)-3-phenylacrylate (1w). Eluent: EtOAc/PE 6:94; $1.5 \mathrm{~h}$; yield: $2.45 \mathrm{~g}, 86 \%$; white solid, mp $76-78{ }^{\circ} \mathrm{C} ;{ }^{1} \mathrm{H}$ NMR $(400 \mathrm{MHz}$, $\left.\mathrm{CDCl}_{3}\right) \delta 8.61(\mathrm{~s}, 1 \mathrm{H}), 7.47-7.44(\mathrm{~m}, 2 \mathrm{H}), 7.40-7.36(\mathrm{~m}, 3 \mathrm{H}), 4.75$ (hept, $J=5.2 \mathrm{~Hz}$, 
$1 \mathrm{H}), 4.61(\mathrm{~s}, 1 \mathrm{H}), 2.52-2.46(\mathrm{~m}, 1 \mathrm{H}), 1.90-1.87(\mathrm{~m}, 2 \mathrm{H}), 1.75-1.72(\mathrm{~m}, 2 \mathrm{H})$, $1.56-1.20(\mathrm{~m}, 6 \mathrm{H}), 0.55-0.47(\mathrm{~m}, 4 \mathrm{H}) ;{ }^{13} \mathrm{C} \mathrm{NMR}\left(100 \mathrm{MHz}, \mathrm{CDCl}_{3}\right) \delta 170.0,165.2$ $136.9,128.9,128.1,127.9,85.8,70.9,32.1,26.8,25.5,24.0,8.6$; HRMS (ESI-TOF) $\mathrm{m} / \mathrm{z}:[\mathrm{M}+\mathrm{H}]^{+}$calcd for $\mathrm{C}_{18} \mathrm{H}_{24} \mathrm{NO}_{2} 286.1802$, found 286.1805 .

Tert-butyl (Z)-3-(cyclopropylamino)-3-phenylacrylate (1x). Eluent: EtOAc/PE 6:94; 2 h; yield: 2.02 g, 78\%; colorless oil; ${ }^{1} \mathrm{H}$ NMR (400 MHz, $\left.\mathrm{CDCl}_{3}\right) \delta 8.53(\mathrm{~s}, 1 \mathrm{H})$, 7.46-7.42 (m, 2H), 7.39-7.36 (m, 3H), $4.54(\mathrm{~s}, 1 \mathrm{H}), 2.49-2.43(\mathrm{~m}, 1 \mathrm{H}), 1.48(\mathrm{~s}, 9 \mathrm{H})$, $0.51-0.50(\mathrm{~m}, 4 \mathrm{H}) ;{ }^{13} \mathrm{C} \mathrm{NMR}\left(100 \mathrm{MHz}, \mathrm{CDCl}_{3}\right) \delta 170.4,164.7,137.0,128.8,128.0$, 127.9, 87.1, 78.4, 28.6, 26.8, 8.6; HRMS (ESI-TOF) m/z: $[\mathrm{M}+\mathrm{H}]^{+}$calcd for $\mathrm{C}_{16} \mathrm{H}_{22} \mathrm{NO}_{2} 260.1645$, found 260.1649.

(Z)-3-(Cyclopropylamino)-1,3-diphenylprop-2-en-1-one (1y). Eluent: EtOAc/PE 2:98; 6 h; yield: 2.26 g, 86\%; white solid, mp 69-71 ${ }^{\circ} \mathrm{C}$; ${ }^{1} \mathrm{H}$ NMR (400 MHz, $\mathrm{CDCl}_{3}$ ) $\delta 11.40(\mathrm{~s}, 1 \mathrm{H}), 7.88(\mathrm{dd}, J=8.4,2.0 \mathrm{~Hz}, 2 \mathrm{H}), 7.56-7.53(\mathrm{~m}, 2 \mathrm{H}), 7.46-7.45(\mathrm{~m}, 3 \mathrm{H})$, 7.43-7.37 (m, 3H), $5.81(\mathrm{~s}, 1 \mathrm{H}), 2.69-2.62(\mathrm{~m}, 1 \mathrm{H}), 0.67-0.65(\mathrm{~m}, 4 \mathrm{H}) ;{ }^{13} \mathrm{C} \mathrm{NMR}$ $\left(100 \mathrm{MHz}, \mathrm{CDCl}_{3}\right) \delta 188.4,167.4,140.2,136.2,130.7,129.4,128.3,128.2,127.9$, 127.0, 93.4, 27.2, 8.7; HRMS (ESI-TOF) $\mathrm{m} / \mathrm{z}:[\mathrm{M}+\mathrm{H}]^{+}$calcd for $\mathrm{C}_{18} \mathrm{H}_{18} \mathrm{NO}$ 264.1383, found 264.1384.

(Z)-3-(cyclopropylamino)- $N$-ethyl-3-phenylacrylamide (1z). Eluent: EtOAc/PE 20:80; 3 h; yield: $1.91 \mathrm{~g}, 83 \%$; white solid, mp $114-116{ }^{\circ} \mathrm{C} ;{ }^{1} \mathrm{H}$ NMR (400 MHz, DMSO-d6) $\delta 9.01(\mathrm{~d}, J=2.4 \mathrm{~Hz}, 1 \mathrm{H}), 7.43-7.40(\mathrm{~m}, 5 \mathrm{H}), 7.30(\mathrm{t}, J=5.2 \mathrm{~Hz}, 1 \mathrm{H})$, $4.52(\mathrm{~s}, 1 \mathrm{H}), 3.10-3.03(\mathrm{~m}, 2 \mathrm{H}), 2.41-2.36(\mathrm{~m}, 1 \mathrm{H}), 1.00(\mathrm{t}, J=7.2 \mathrm{~Hz}, 3 \mathrm{H})$, 0.45-0.31 (m, 4H); ${ }^{13} \mathrm{C}$ NMR (100 MHz, DMSO-d6) $\delta 169.9,160.7,137.8,129.1$, 128.6, 128.1, 90.4, 33.2, 26.6, 15.6 , 8.7; HRMS (ESI-TOF) m/z: $[\mathrm{M}+\mathrm{H}]^{+}$calcd for $\mathrm{C}_{14} \mathrm{H}_{19} \mathrm{~N}_{2} \mathrm{O} 231.1492$, found 231.1489. 
3. General Procedure for the Synthesis of 1,4-Dihydropyridines 2. A solution of the enamine substrate $(\mathbf{1})(0.5 \mathrm{mmol})$ in DCE $(10 \mathrm{~mL})$ was treated sequentially with iodine (152 mg, $0.6 \mathrm{mmol})$ and $\mathrm{NH}_{4} \mathrm{PF}_{6}(245 \mathrm{mg}, 1.5 \mathrm{mmol})$. The reaction mixture was stirred at $0{ }^{\circ} \mathrm{C}$ (at $-20{ }^{\circ} \mathrm{C}$ for $\mathbf{1 h}$; at $50{ }^{\circ} \mathrm{C}$ for $\mathbf{1 n}$ with a heating mantle) for $1 \mathrm{~h}$, and then at room temperature $\left(25^{\circ} \mathrm{C}\right.$; at the reflux temperature for $\mathbf{1 g}$ and $\mathbf{1 h}$ with a heating mantle; at $50{ }^{\circ} \mathrm{C}$ for $\mathbf{1 n}$ with a heating mantle). Upon completion of the reaction as indicated by TLC, it was quenched with sat. $\mathrm{Na}_{2} \mathrm{~S}_{2} \mathrm{O}_{3}(2 \mathrm{~mL})$, diluted with water $(10 \mathrm{~mL})$, and extracted with $\mathrm{CH}_{2} \mathrm{Cl}_{2}(10 \mathrm{~mL} \times 3)$. The combined organic layer was dried over anhydrous $\mathrm{Na}_{2} \mathrm{SO}_{4}$, concentrated and purified through silica gel column chromatography to afford the 1,4-dihydropyridine product (2).

Dimethyl 4-(2-iodoethyl)-2,6-diphenyl-1,4-dihydropyridine-3,5-dicarboxylate (2a). Eluent: $\mathrm{CH}_{2} \mathrm{Cl}_{2} / \mathrm{PE} \mathrm{67:33;} 5$ h; yield: $98 \mathrm{mg}, 78 \%$; white solid, mp 192-194 ${ }^{\circ} \mathrm{C}$; ${ }^{1} \mathrm{H}$ NMR (400 MHz, $\left.\mathrm{CDCl}_{3}\right) \delta$ 7.44-7.41 (m, 6H), 7.37-7.34 (m, 4H), $6.14(\mathrm{~s}, 1 \mathrm{H})$, $4.14(\mathrm{t}, J=6.4 \mathrm{~Hz}, 1 \mathrm{H}), 3.53(\mathrm{~s}, 6 \mathrm{H}), 3.36-3.32(\mathrm{~m}, 2 \mathrm{H}), 2.32-2.27(\mathrm{~m}, 2 \mathrm{H}) ;{ }^{13} \mathrm{C}$ NMR (100 MHz, $\left.\mathrm{CDCl}_{3}\right) \delta 167.1,147.4,136.0,129.6,128.5,128.2,101.6,51.2,41.5$, 35.7, 1.8; HRMS (ESI-TOF) m/z: $[\mathrm{M}+\mathrm{Na}]^{+}$calcd for $\mathrm{C}_{23} \mathrm{H}_{22} \mathrm{INNaO}_{4}$ 526.0486, found 526.0487 .

\section{Dimethyl 4-(2-iodoethyl)-2,6-di-p-tolyl-1,4-dihydropyridine-3,5-dicarboxylate} (2b). Eluent: $\mathrm{CH}_{2} \mathrm{Cl}_{2} / \mathrm{PE} 67: 33$; 5 h; yield: $89 \mathrm{mg}, 67 \%$; white solid, mp $136-138{ }^{\circ} \mathrm{C}$; ${ }^{1} \mathrm{H}$ NMR $\left(400 \mathrm{MHz}, \mathrm{CDCl}_{3}\right) \delta 7.26-7.20(\mathrm{~m}, 8 \mathrm{H}$, overlapped with the peak of chloroform), $6.14(\mathrm{~s}, 1 \mathrm{H}), 4.11(\mathrm{t}, J=6.0 \mathrm{~Hz}, 1 \mathrm{H}), 3.55(\mathrm{~s}, 6 \mathrm{H}), 3.33(\mathrm{t}, J=8.8 \mathrm{~Hz}$, 2H), $2.38(\mathrm{~s}, 6 \mathrm{H}), 2.30-2.25(\mathrm{~m}, 2 \mathrm{H}) ;{ }^{13} \mathrm{C} \mathrm{NMR}\left(100 \mathrm{MHz}, \mathrm{CDCl}_{3}\right) \delta$ 167.2, 147.5, 139.7, 133.0, 129.1, 128.2, 101.2, 51.2, 41.5, 35.7, 21.4, 2.1; HRMS (ESI-TOF) m/z: $[\mathrm{M}+\mathrm{H}]^{+}$calcd for $\mathrm{C}_{25} \mathrm{H}_{27} \mathrm{INO}_{4} 532.0979$, found 532.0978. 
Dimethyl 4-(2-iodoethyl)-2,6-bis(4-methoxyphenyl)-1,4-dihydropyridine-3,5-dicarboxylate (2c). Eluent: $\mathrm{CH}_{2} \mathrm{Cl}_{2} / \mathrm{PE} 67: 33 ; 7$ h; yield: $87 \mathrm{mg}, 62 \%$; white solid, mp 103-105 ${ }^{\circ} \mathrm{C} ;{ }^{1} \mathrm{H}$ NMR $\left(400 \mathrm{MHz}, \mathrm{CDCl}_{3}\right) \delta 7.32-7.30(\mathrm{~m}, 4 \mathrm{H}), 6.93(\mathrm{~d}, J=8.4 \mathrm{~Hz}$, 4H), $6.19(\mathrm{~s}, 1 \mathrm{H}), 4.09(\mathrm{t}, J=6.4 \mathrm{~Hz}, 1 \mathrm{H}), 3.84(\mathrm{~s}, 6 \mathrm{H}), 3.56(\mathrm{~s}, 6 \mathrm{H}), 3.35-3.31(\mathrm{~m}$, 2H), 2.29-2.24 (m, 2H); ${ }^{13} \mathrm{C}$ NMR (100 MHz, $\left.\mathrm{CDCl}_{3}\right) \delta 167.3,160.6,147.1,129.8$, 128.0, 113.8, 101.0, 55.3, 51.2, 41.3, 35.7, 2.2; HRMS (ESI-TOF) m/z: $[\mathrm{M}+\mathrm{H}]^{+}$ calcd for $\mathrm{C}_{25} \mathrm{H}_{27} \mathrm{INO}_{6} 564.0878$, found 564.0879.

Dimethyl 2,6-bis(4-fluorophenyl)-4-(2-iodoethyl)-1,4-dihydropyridine-3,5-dicarboxylate (2d). Eluent: $\mathrm{CH}_{2} \mathrm{Cl}_{2} / \mathrm{PE} 67: 33$; 4.5 h; yield: $97 \mathrm{mg}, 72 \%$; white solid, mp $200-202{ }^{\circ} \mathrm{C} ;{ }^{1} \mathrm{H}$ NMR (400 MHz, $\left.\mathrm{CDCl}_{3}\right) \delta$ 7.37-7.32 (m, 4H), 7.14-7.09 (m, 4H), $6.07(\mathrm{~s}, 1 \mathrm{H}), 4.13(\mathrm{t}, J=6.4 \mathrm{~Hz}, 1 \mathrm{H}), 3.55(\mathrm{~s}, 6 \mathrm{H}), 3.33-3.29(\mathrm{~m}, 2 \mathrm{H}), 2.30-2.24$ $(\mathrm{m}, 2 \mathrm{H}) ;{ }^{13} \mathrm{C} \mathrm{NMR}\left(100 \mathrm{MHz}, \mathrm{CDCl}_{3}\right) \delta 166.9,163.4\left(\mathrm{~d}, J_{\mathrm{C}-\mathrm{F}}=248.6 \mathrm{~Hz}\right), 146.2$, $131.8\left(\mathrm{~d}, J_{\mathrm{C}-\mathrm{F}}=3.5 \mathrm{~Hz}\right), 130.3\left(\mathrm{~d}, J_{\mathrm{C}-\mathrm{F}}=8.5 \mathrm{~Hz}\right), 115.7\left(\mathrm{~d}, J_{\mathrm{C}-\mathrm{F}}=21.7 \mathrm{~Hz}\right), 102.2$, 51.4, 41.4, 35.6, 1.5; ${ }^{19} \mathrm{~F}$ NMR (376 MHz, $\left.\mathrm{CDCl}_{3}\right) \delta-110.5$; HRMS (ESI-TOF) m/z: $[\mathrm{M}+\mathrm{Na}]^{+}$calcd for $\mathrm{C}_{23} \mathrm{H}_{20} \mathrm{~F}_{2} \mathrm{INNaO}_{4}$ 562.0297, found 562.0299.

Dimethyl 2,6-bis(4-chlorophenyl)-4-(2-iodoethyl)-1,4-dihydropyridine-3,5-dicarboxylate (2e). Eluent: $\mathrm{CH}_{2} \mathrm{Cl}_{2} / \mathrm{PE} 67: 33 ; 6$ h; yield: $85 \mathrm{mg}, 60 \%$; white solid, mp $170-172{ }^{\circ} \mathrm{C} ;{ }^{1} \mathrm{H}$ NMR $\left(400 \mathrm{MHz}, \mathrm{CDCl}_{3}\right) \delta 7.39(\mathrm{~d}, J=8.0 \mathrm{~Hz}, 4 \mathrm{H}), 7.28(\mathrm{~d}, J=8.4$ $\mathrm{Hz}, 4 \mathrm{H}$, overlapped with the peak of chloroform), $6.08(\mathrm{~s}, 1 \mathrm{H}), 4.13(\mathrm{t}, J=6.0 \mathrm{~Hz}$, 1H), $3.55(\mathrm{~s}, 6 \mathrm{H}), 3.29(\mathrm{t}, J=8.4 \mathrm{~Hz}, 2 \mathrm{H}), 2.29-2.23(\mathrm{~m}, 2 \mathrm{H}) ;{ }^{13} \mathrm{C}$ NMR $(100 \mathrm{MHz}$, $\left.\mathrm{CDCl}_{3}\right) \delta 166.8,146.0,135.8,134.2,129.7,128.8,102.3,51.4,41.4,35.6,1.4 ;$ HRMS (ESI-TOF) m/z: $[\mathrm{M}+\mathrm{Na}]^{+}$calcd for $\mathrm{C}_{23} \mathrm{H}_{20} \mathrm{Cl}_{2} \mathrm{INNaO}_{4}$ 593.9706, found 593.9707 .

Dimethyl 2,6-bis(4-bromophenyl)-4-(2-iodoethyl)-1,4-dihydropyridine-3,5-dicarboxylate (2f). Eluent: $\mathrm{CH}_{2} \mathrm{Cl}_{2} / \mathrm{PE} \mathrm{67:33;} 6$ h; yield: $97 \mathrm{mg}$, 59\%; white solid, mp $163-166{ }^{\circ} \mathrm{C} ;{ }^{1} \mathrm{H}$ NMR $\left(400 \mathrm{MHz}, \mathrm{CDCl}_{3}\right) \delta 7.55(\mathrm{dt}, J=8.8,2.0 \mathrm{~Hz}, 4 \mathrm{H}), 7.21(\mathrm{dt}, J$ 
$=8.8,2.0 \mathrm{~Hz}, 4 \mathrm{H}), 6.04(\mathrm{~s}, 1 \mathrm{H}), 4.13(\mathrm{t}, J=6.0 \mathrm{~Hz}, 1 \mathrm{H}), 3.56(\mathrm{~s}, 6 \mathrm{H}), 3.31-3.27(\mathrm{~m}$, 2H), 2.28-2.23 (m, 2H); ${ }^{13} \mathrm{C} \mathrm{NMR}\left(100 \mathrm{MHz}, \mathrm{CDCl}_{3}\right) \delta 166.7,146.0,134.7,131.8$, 129.9, 124.0, 102.3, 51.4, 41.4, 35.5, 1.3; HRMS (ESI-TOF) m/z: $[\mathrm{M}+\mathrm{H}]^{+}$calcd for $\mathrm{C}_{23} \mathrm{H}_{21} \mathrm{Br}_{2} \mathrm{INO}_{4} 659.8877$, found 659.8875 .

Dimethyl 4-(2-iodoethyl)-2,6-bis(4-(trifluoromethyl)phenyl)-1,4-dihydropyridine3,5-dicarboxylate (2g). Eluent: $\mathrm{CH}_{2} \mathrm{Cl}_{2} / \mathrm{PE}$ 67:33; 5 h; yield: 89 mg, 56\%; white solid, mp $127-129{ }^{\circ} \mathrm{C} ;{ }^{1} \mathrm{H}$ NMR $\left(400 \mathrm{MHz}, \mathrm{CDCl}_{3}\right) \delta 7.69(\mathrm{~d}, J=8.4 \mathrm{~Hz}, 4 \mathrm{H}), 7.47(\mathrm{~d}, J=$ $8.0 \mathrm{~Hz}, 4 \mathrm{H}), 6.01(\mathrm{~s}, 1 \mathrm{H}), 4.20(\mathrm{t}, J=6.0 \mathrm{~Hz}, 1 \mathrm{H}), 3.56(\mathrm{~s}, 6 \mathrm{H}), 3.31(\mathrm{t}, J=8.4 \mathrm{~Hz}$, 2H), 2.33-2.27 (m, 2H); ${ }^{13} \mathrm{C} \mathrm{NMR}\left(100 \mathrm{MHz}, \mathrm{CDCl}_{3}\right) \delta 166.5,145.8,139.4$ (q, $J_{\mathrm{C}-\mathrm{F}}=$ $1.4 \mathrm{~Hz}), 131.7\left(\mathrm{q}, J_{\mathrm{C}-\mathrm{F}}=32.7 \mathrm{~Hz}\right), 128.7,125.6\left(\mathrm{q}, J_{\mathrm{C}-\mathrm{F}}=3.8 \mathrm{~Hz}\right), 123.7\left(\mathrm{q}, J_{\mathrm{C}-\mathrm{F}}=\right.$ $270.9 \mathrm{~Hz}), 103.1,51.5,41.4,35.5,1.0 ;{ }^{19} \mathrm{~F}$ NMR (376 MHz, $\left.\mathrm{CDCl}_{3}\right) \delta-62.8$; HRMS (ESI-TOF) m/z: $[\mathrm{M}+\mathrm{H}]^{+}$calcd for $\mathrm{C}_{25} \mathrm{H}_{21} \mathrm{~F}_{6} \mathrm{INO}_{4} 640.0414$, found 640.0409 .

Dimethyl 2,6-bis(4-cyanophenyl)-4-(2-iodoethyl)-1,4-dihydropyridine-3,5-dicarboxylate (2h). Eluent: $\mathrm{CH}_{2} \mathrm{Cl}_{2} / \mathrm{PE} 67: 33 ; 8 \mathrm{~h}\left(-20^{\circ} \mathrm{C}\right.$ for $3 \mathrm{~h}$, reflux for $\left.5 \mathrm{~h}\right)$; yield: 73 mg, 53\%; yellow solid, mp $178-180{ }^{\circ} \mathrm{C} ;{ }^{1} \mathrm{H}$ NMR (400 MHz, $\left.\mathrm{CDCl}_{3}\right) \delta 7.69(\mathrm{~d}, J=$ $8.0 \mathrm{~Hz}, 4 \mathrm{H}), 7.47(\mathrm{~d}, J=8.0 \mathrm{~Hz}, 4 \mathrm{H}), 6.24(\mathrm{~s}, 1 \mathrm{H}), 4.18(\mathrm{t}, J=6.0 \mathrm{~Hz}, 1 \mathrm{H}), 3.56(\mathrm{~s}$, $6 \mathrm{H}), 3.28(\mathrm{t}, J=8.4 \mathrm{~Hz}, 2 \mathrm{H}), 2.30-2.25(\mathrm{~m}, 2 \mathrm{H}) ;{ }^{13} \mathrm{C} \mathrm{NMR}\left(100 \mathrm{MHz}, \mathrm{CDCl}_{3}\right) \delta$ $166.3,145.3,140.2,132.2,129.3,118.1,113.4,103.4,51.6,41.4,35.4,0.7$; HRMS (ESI-TOF) m/z: [M + H] $]^{+}$calcd for $\mathrm{C}_{25} \mathrm{H}_{21} \mathrm{IN}_{3} \mathrm{O}_{4}$ 554.0571, found 554.0570.

\section{Dimethyl 4-(2-iodoethyl)-2,6-di-m-tolyl-1,4-dihydropyridine-3,5-dicarboxylate} (2i). Eluent: $\mathrm{CH}_{2} \mathrm{Cl}_{2} / \mathrm{PE} 67: 33$; 5 h; yield: $90 \mathrm{mg}$, 68\%; white solid, mp $167-169{ }^{\circ} \mathrm{C}$; ${ }^{1} \mathrm{H}$ NMR $\left(400 \mathrm{MHz}, \mathrm{CDCl}_{3}\right) \delta 7.30(\mathrm{t}, J=8.0 \mathrm{~Hz}, 2 \mathrm{H}), 7.23(\mathrm{~d}, J=7.6 \mathrm{~Hz}, 2 \mathrm{H}), 7.14$ $(\mathrm{d}, J=7.2 \mathrm{~Hz}, 4 \mathrm{H}), 6.10(\mathrm{~s}, 1 \mathrm{H}), 4.12(\mathrm{t}, J=6.0 \mathrm{~Hz}, 1 \mathrm{H}), 3.54(\mathrm{~s}, 6 \mathrm{H}), 3.36-3.32(\mathrm{~m}$, 2H), $2.39(\mathrm{~s}, 6 \mathrm{H}), 2.32-2.26(\mathrm{~m}, 2 \mathrm{H}) ;{ }^{13} \mathrm{C} \mathrm{NMR}\left(100 \mathrm{MHz}, \mathrm{CDCl}_{3}\right) \delta 167.2,147.6$, 
138.2, 136.0, 130.3, 128.6, 128.3, 125.3, 101.5, 51.2, 41.6, 35.8, 21.4, 2.0; HRMS (ESI-TOF) m/z: $[\mathrm{M}+\mathrm{H}]^{+}$calcd for $\mathrm{C}_{25} \mathrm{H}_{27} \mathrm{INO}_{4}$ 532.0979, found 532.0978.

Dimethyl 2,6-bis(2-fluorophenyl)-4-(2-iodoethyl)-1,4-dihydropyridine-3,5-dicarboxylate (2j). Eluent: $\mathrm{CH}_{2} \mathrm{Cl}_{2} / \mathrm{PE}$ 67:33; 6 h; yield: $86 \mathrm{mg}, 64 \%$; white solid, mp $198-200{ }^{\circ} \mathrm{C} ;{ }^{1} \mathrm{H} \mathrm{NMR}\left(400 \mathrm{MHz}, \mathrm{CDCl}_{3}\right) \delta 7.43-7.38(\mathrm{~m}, 2 \mathrm{H}), 7.30$ (t, $J=7.6 \mathrm{~Hz}$, 2H), $7.20(\mathrm{t}, J=7.6 \mathrm{~Hz}, 2 \mathrm{H}), 7.12(\mathrm{t}, J=9.2 \mathrm{~Hz}, 2 \mathrm{H}), 6.05(\mathrm{~s}, 1 \mathrm{H}), 4.21(\mathrm{t}, J=6.0 \mathrm{~Hz}$, 1H), $3.57(\mathrm{~s}, 6 \mathrm{H}), 3.33(\mathrm{t}, J=8.4 \mathrm{~Hz}, 2 \mathrm{H}), 2.32-2.26(\mathrm{~m}, 2 \mathrm{H}) ;{ }^{13} \mathrm{C}$ NMR $(100 \mathrm{MHz}$, $\left.\mathrm{CDCl}_{3}\right) \delta 166.5,159.6\left(\mathrm{~d}, J_{\mathrm{C}-\mathrm{F}}=247.7 \mathrm{~Hz}\right), 141.1,131.4\left(\mathrm{~d}, J_{\mathrm{C}-\mathrm{F}}=8.3 \mathrm{~Hz}\right), 129.8$, $124.3\left(\mathrm{~d}, J_{\mathrm{C}-\mathrm{F}}=3.6 \mathrm{~Hz}\right), 123.7\left(\mathrm{~d}, J_{\mathrm{C}-\mathrm{F}}=15.2 \mathrm{~Hz}\right), 115.8\left(\mathrm{~d}, J_{\mathrm{C}-\mathrm{F}}=21.5 \mathrm{~Hz}\right), 103.9$, 51.4, 41.1, 35.5, 1.5; ${ }^{19} \mathrm{~F}$ NMR (376 MHz, $\left.\mathrm{CDCl}_{3}\right) \delta-114.7 ; \mathrm{HRMS}$ (ESI-TOF) m/z: $[\mathrm{M}+\mathrm{H}]^{+}$calcd for $\mathrm{C}_{23} \mathrm{H}_{21} \mathrm{~F}_{2} \mathrm{INO}_{4} 540.0478$, found 540.0477 .

Dimethyl 2,6-bis(3,4-dimethylphenyl)-4-(2-iodoethyl)-1,4-dihydropyridine-3,5dicarboxylate (2k). Eluent: $\mathrm{CH}_{2} \mathrm{Cl}_{2} / \mathrm{PE} 67: 33 ; 4$ h; yield: $80 \mathrm{mg}, 57 \%$; white solid, mp $135-137{ }^{\circ} \mathrm{C} ;{ }^{1} \mathrm{H}$ NMR $\left(400 \mathrm{MHz}, \mathrm{CDCl}_{3}\right) \delta 7.16(\mathrm{~d}, J=7.6 \mathrm{~Hz}, 2 \mathrm{H}), 7.08(\mathrm{~d}, J=$ $7.2 \mathrm{~Hz}, 4 \mathrm{H}), 6.10(\mathrm{~s}, 1 \mathrm{H}), 4.10(\mathrm{t}, J=6.0 \mathrm{~Hz}, 1 \mathrm{H}), 3.56(\mathrm{~s}, 6 \mathrm{H}), 3.36-3.32(\mathrm{~m}, 2 \mathrm{H})$, 2.30-2.25 (m, 14H); ${ }^{13} \mathrm{C}$ NMR (100 MHz, $\left.\mathrm{CDCl}_{3}\right) \delta 167.3,147.7,138.3,136.8,133.5$, 129.7, 129.1, 125.7, 101.0, 51.2, 41.5, 35.8, 19.83, 19.75, 2.3; HRMS (ESI-TOF) m/z: $[\mathrm{M}+\mathrm{H}]^{+}$calcd for $\mathrm{C}_{27} \mathrm{H}_{31} \mathrm{INO}_{4} 560.1292$, found 560.1297 .

Dimethyl 2,6-bis(benzo[d][1,3]dioxol-5-yl)-4-(2-iodoethyl)-1,4-dihydropyridine3,5-dicarboxylate (2l). Eluent: $\mathrm{CH}_{2} \mathrm{Cl}_{2} / \mathrm{PE} \mathrm{67:33;} 5$ h; yield: $94 \mathrm{mg}, 64 \%$; white solid, mp $172-174{ }^{\circ} \mathrm{C} ;{ }^{1} \mathrm{H}$ NMR $\left(400 \mathrm{MHz}, \mathrm{CDCl}_{3}\right) \delta 6.82(\mathrm{~d}, J=10.4 \mathrm{~Hz}, 6 \mathrm{H}), 6.19$ (s, 1H), $6.01(\mathrm{~s}, 4 \mathrm{H}), 4.06(\mathrm{t}, J=6.0 \mathrm{~Hz}, 1 \mathrm{H}), 3.58(\mathrm{~s}, 6 \mathrm{H}), 3.30(\mathrm{t}, J=8.4 \mathrm{~Hz}, 2 \mathrm{H})$, 2.26-2.21 (m, 2H); $\left.{ }^{13} \mathrm{C} \mathrm{NMR} \mathrm{(100} \mathrm{MHz,} \mathrm{CDCl}_{3}\right) \delta 167.0,148.8,147.6,146.7,129.4$, 122.2, 109.0, 108.3, 101.5, 101.2, 51.3, 41.2, 35.6, 1.9; HRMS (ESI-TOF) m/z: [M + $\mathrm{H}]^{+}$calcd for $\mathrm{C}_{25} \mathrm{H}_{23} \mathrm{INO}_{8} 592.0463$, found 592.0466. 
Dimethyl 4-(2-iodoethyl)-2,6-di(naphthalen-2-yl)-1,4-dihydropyridine-3,5-dicarboxylate (2m). Eluent: $\mathrm{CH}_{2} \mathrm{Cl}_{2} / \mathrm{PE}$ 67:33; 5 h; yield: $107 \mathrm{mg}, 71 \%$; white solid, mp $170-172{ }^{\circ} \mathrm{C} ;{ }^{1} \mathrm{H}$ NMR $\left(400 \mathrm{MHz}, \mathrm{CDCl}_{3}\right) \delta 7.88-7.85(\mathrm{~m}, 8 \mathrm{H}), 7.55-7.50(\mathrm{~m}$, 4H), $7.45(\mathrm{dd}, J=8.4,1.6 \mathrm{~Hz}, 2 \mathrm{H}), 6.33(\mathrm{~s}, 1 \mathrm{H}), 4.22(\mathrm{t}, J=6.0 \mathrm{~Hz}, 1 \mathrm{H}), 3.52(\mathrm{~s}, 6 \mathrm{H})$, $3.42(\mathrm{t}, J=8.4 \mathrm{~Hz}, 2 \mathrm{H}), 2.41-2.36(\mathrm{~m}, 2 \mathrm{H}) ;{ }^{13} \mathrm{C} \mathrm{NMR}\left(100 \mathrm{MHz}, \mathrm{CDCl}_{3}\right) \delta 167.1$, $147.4,133.6,132.8,128.2,128.0,127.8,127.09,127.06,126.6,126.3,101.9,51.3$, 41.6, 35.9, 2.0; HRMS (ESI-TOF) m/z: [M + Na] ${ }^{+}$calcd for $\mathrm{C}_{31} \mathrm{H}_{26} \mathrm{INNaO}_{4}$ 626.0799, found 626.0801 .

Dimethyl 4-(2-iodoethyl)-2,6-di(thiophen-2-yl)-1,4-dihydropyridine-3,5-dicarboxylate (2n). Eluent: $\mathrm{CH}_{2} \mathrm{Cl}_{2} / \mathrm{PE} 67: 33 ; 6$ h; yield: $33 \mathrm{mg}, 26 \%$; white solid, mp 157-159 ${ }^{\circ} \mathrm{C} ;{ }^{1} \mathrm{H}$ NMR $\left(400 \mathrm{MHz}, \mathrm{CDCl}_{3}\right) \delta 7.46(\mathrm{dd}, J=5.2,1.2 \mathrm{~Hz}, 2 \mathrm{H}), 7.27(\mathrm{dd}, J$ $=3.6,1.2 \mathrm{~Hz}, 2 \mathrm{H}$, overlapped with the peak of chloroform), 7.08 (dd, $J=5.2,4.0 \mathrm{~Hz}$, 2H), $6.45(\mathrm{~s}, 1 \mathrm{H}), 4.08(\mathrm{t}, J=6.4 \mathrm{~Hz}, 1 \mathrm{H}), 3.62(\mathrm{~s}, 6 \mathrm{H}), 3.30(\mathrm{t}, J=8.4 \mathrm{~Hz}, 2 \mathrm{H})$, 2.27-2.21 (m, 2H); $\left.{ }^{13} \mathrm{C} \mathrm{NMR} \mathrm{(100} \mathrm{MHz,} \mathrm{CDCl}_{3}\right) \delta 166.8,139.4,135.9,129.2,128.2$, 127.3, 102.9, 51.5, 40.3, 36.3, 1.8; HRMS (ESI-TOF) m/z: $[\mathrm{M}+\mathrm{H}]^{+}$calcd for $\mathrm{C}_{19} \mathrm{H}_{19} \mathrm{INO}_{4} \mathrm{~S}_{2}$ 515.9795, found 515.9794.

Dibenzyl 4-(2-iodoethyl)-2,6-diphenyl-1,4-dihydropyridine-3,5-dicarboxylate (2o). Eluent: $\mathrm{CH}_{2} \mathrm{Cl}_{2} / \mathrm{PE}$ 67:33; 4 h; yield: $106 \mathrm{mg}, 65 \%$; white solid, mp $119-121{ }^{\circ} \mathrm{C} ;{ }^{1} \mathrm{H}$ NMR (400 MHz, $\left.\mathrm{CDCl}_{3}\right) \delta 7.38-7.30(\mathrm{~m}, 10 \mathrm{H}), 7.23-7.19(\mathrm{~m}, 6 \mathrm{H}), 6.97-6.95(\mathrm{~m}$, 4H), $6.17(\mathrm{~s}, 1 \mathrm{H}), 5.00-4.91(\mathrm{~m}, 4 \mathrm{H}), 4.17(\mathrm{t}, J=6.0 \mathrm{~Hz}, 1 \mathrm{H}), 3.33-3.29(\mathrm{~m}, 2 \mathrm{H})$, 2.34-2.29 (m, 2H); ${ }^{13} \mathrm{C}$ NMR (100 MHz, $\left.\mathrm{CDCl}_{3}\right) \delta 166.5,147.2,136.0,135.8,129.5$, 128.5, 128.3, 128.2, 127.8, 127.7, 101.6, 65.9, 41.6, 36.0, 1.8; HRMS (ESI-TOF) m/z: $[\mathrm{M}+\mathrm{Na}]^{+}$calcd for $\mathrm{C}_{35} \mathrm{H}_{30} \mathrm{INNaO}_{4} 678.1112$, found 678.1114 .

Bis(4-methoxybenzyl) 4-(2-iodoethyl)-2,6-diphenyl-1,4-dihydropyridine-3,5-dicarboxylate (2p). Eluent: $\mathrm{CH}_{2} \mathrm{Cl}_{2} / \mathrm{PE} \mathrm{67:33;} 5$ h; yield: $115 \mathrm{mg}, 64 \%$; white solid, mp 
87-89 ${ }^{\circ} \mathrm{C} ;{ }^{1} \mathrm{H}$ NMR $\left(400 \mathrm{MHz}, \mathrm{CDCl}_{3}\right) \delta 7.38-7.29(\mathrm{~m}, 10 \mathrm{H}), 6.92(\mathrm{~d}, J=8.4 \mathrm{~Hz}$, 4H), $6.76(\mathrm{~d}, J=8.4 \mathrm{~Hz}, 4 \mathrm{H}), 6.11(\mathrm{~s}, 1 \mathrm{H}), 4.93-4.85(\mathrm{~m}, 4 \mathrm{H}), 4.13(\mathrm{t}, J=6.0 \mathrm{~Hz}$, 1H), $3.79(\mathrm{~s}, 6 \mathrm{H}), 3.29(\mathrm{t}, J=8.4 \mathrm{~Hz}, 2 \mathrm{H}), 2.32-2.26(\mathrm{~m}, 2 \mathrm{H}) ;{ }^{13} \mathrm{C}$ NMR $(100 \mathrm{MHz}$, $\left.\mathrm{CDCl}_{3}\right) \delta 166.6,159.2,147.1,136.1,129.7,129.5,128.5,128.3,128.0,113.6,101.8$ 65.7, 55.2, 41.6, 36.0, 2.0; HRMS (ESI-TOF) m/z: $[\mathrm{M}+\mathrm{H}]^{+}$calcd for $\mathrm{C}_{37} \mathrm{H}_{35} \mathrm{INO}_{6}$ 716.1504 , found 716.1502 .

Bis(4-chlorobenzyl) 4-(2-iodoethyl)-2,6-diphenyl-1,4-dihydropyridine-3,5-dicarboxylate (2q). Eluent: $\mathrm{CH}_{2} \mathrm{Cl}_{2} / \mathrm{PE} 67: 33 ; 4$ h; yield: $116 \mathrm{mg}, 64 \%$; white solid, mp 54-56 ${ }^{\circ} \mathrm{C} ;{ }^{1} \mathrm{H}$ NMR $\left(400 \mathrm{MHz}, \mathrm{CDCl}_{3}\right) \delta 7.39-7.30(\mathrm{~m}, 10 \mathrm{H}), 7.18(\mathrm{~d}, J=8.4 \mathrm{~Hz}$, 4H), $6.86(\mathrm{~d}, J=8.4 \mathrm{~Hz}, 4 \mathrm{H}), 6.16(\mathrm{~s}, 1 \mathrm{H}), 4.95-4.88(\mathrm{~m}, 4 \mathrm{H}), 4.15(\mathrm{t}, J=6.4 \mathrm{~Hz}$, 1H), $3.32(\mathrm{t}, J=8.4 \mathrm{~Hz}, 2 \mathrm{H}), 2.33-2.28(\mathrm{~m}, 2 \mathrm{H}) ;{ }^{13} \mathrm{C} \mathrm{NMR}\left(100 \mathrm{MHz}, \mathrm{CDCl}_{3}\right) \delta$ $166.4,147.3,136.0,134.2,133.6,129.7,129.2,128.6,128.4,128.3,101.5,65.2,41.5$, 35.9, 1.7; HRMS (ESI-TOF) m/z: $[\mathrm{M}+\mathrm{H}]^{+}$calcd for $\mathrm{C}_{35} \mathrm{H}_{29} \mathrm{Cl}_{2} \mathrm{INO}_{4} 724.0513$, found 724.0511.

Bis(4-bromobenzyl) 4-(2-iodoethyl)-2,6-diphenyl-1,4-dihydropyridine-3,5-dicarboxylate (2r). Eluent: $\mathrm{CH}_{2} \mathrm{Cl}_{2} / \mathrm{PE} \mathrm{67:33;} 4 \mathrm{~h}$; yield: $114 \mathrm{mg}, 56 \%$; white solid, mp 61-63 ${ }^{\circ} \mathrm{C} ;{ }^{1} \mathrm{H}$ NMR (400 MHz, $\left.\mathrm{CDCl}_{3}\right) \delta 7.42-7.30(\mathrm{~m}, 14 \mathrm{H}), 6.80(\mathrm{~d}, J=8.4 \mathrm{~Hz}$, 4H), $6.16(\mathrm{~s}, 1 \mathrm{H}), 4.94-4.86(\mathrm{~m}, 4 \mathrm{H}), 4.15(\mathrm{t}, J=6.0 \mathrm{~Hz}, 1 \mathrm{H}), 3.34-3.29(\mathrm{~m}, 2 \mathrm{H})$, 2.33-2.28 (m, 2H); $\left.{ }^{13} \mathrm{C} \mathrm{NMR} \mathrm{(100} \mathrm{MHz,} \mathrm{CDCl}_{3}\right) \delta 166.3,147.3,136.0,134.8,131.3$, 129.7, 129.5, 128.6, 128.3, 121.8, 101.5, 65.2, 41.5, 35.9, 1.7; HRMS (ESI-TOF) m/z: $[\mathrm{M}+\mathrm{H}]^{+}$calcd for $\mathrm{C}_{35} \mathrm{H}_{29} \mathrm{Br}_{2} \mathrm{INO}_{4}$ 811.9503, found 811.9500.

Diethyl 4-(2-iodoethyl)-2,6-diphenyl-1,4-dihydropyridine-3,5-dicarboxylate (2s). Eluent: $\mathrm{CH}_{2} \mathrm{Cl}_{2} / \mathrm{PE}$ 67:33; $5 \mathrm{~h}$; yield: $82 \mathrm{mg}, 62 \%$; white solid, mp $153-155{ }^{\circ} \mathrm{C}$; ${ }^{1} \mathrm{H}$ NMR (400 MHz, $\left.\mathrm{CDCl}_{3}\right) \delta$ 7.42-7.38 (m, 6H), 7.38-7.34 (m, 4H), $6.10(\mathrm{~s}, 1 \mathrm{H}), 4.12$ $(\mathrm{t}, J=6.0 \mathrm{~Hz}, 1 \mathrm{H}), 3.96(\mathrm{q}, J=7.2 \mathrm{~Hz}, 4 \mathrm{H}), 3.38-3.34(\mathrm{~m}, 2 \mathrm{H}), 2.34-2.28(\mathrm{~m}, 2 \mathrm{H})$, 
$0.95(\mathrm{t}, J=7.2 \mathrm{~Hz}, 6 \mathrm{H}) ;{ }^{13} \mathrm{C} \mathrm{NMR}\left(100 \mathrm{MHz}, \mathrm{CDCl}_{3}\right) \delta 166.8,147.0,136.4,129.5$, 128.4, 128.3, 102.1, 60.0, 41.7, 35.8, 13.8, 2.1; HRMS (ESI-TOF) m/z: $[\mathrm{M}+\mathrm{H}]^{+}$ calcd for $\mathrm{C}_{25} \mathrm{H}_{27} \mathrm{INO}_{4} 532.0979$, found 532.0983.

Dihexyl 4-(2-iodoethyl)-2,6-diphenyl-1,4-dihydropyridine-3,5-dicarboxylate (2t). Eluent: $\mathrm{CH}_{2} \mathrm{Cl}_{2} / \mathrm{PE}$ 67:33; $4 \mathrm{~h}$; yield: $103 \mathrm{mg}, 64 \%$; white solid, mp $68-70{ }^{\circ} \mathrm{C} ;{ }^{1} \mathrm{H}$ NMR (400 MHz, $\left.\mathrm{CDCl}_{3}\right) \delta 7.41-7.36(\mathrm{~m}, 10 \mathrm{H}), 6.08(\mathrm{~s}, 1 \mathrm{H}), 4.13(\mathrm{~s}, 1 \mathrm{H}), 3.91(\mathrm{~d}, J$ $=5.6 \mathrm{~Hz}, 4 \mathrm{H}), 3.36(\mathrm{t}, J=8.0 \mathrm{~Hz}, 2 \mathrm{H}), 2.31(\mathrm{~d}, J=7.2 \mathrm{~Hz}, 2 \mathrm{H}), 1.30-1.04(\mathrm{~m}, 16 \mathrm{H})$, $0.86(\mathrm{t}, J=6.4 \mathrm{~Hz}, 6 \mathrm{H}) ;{ }^{13} \mathrm{C} \mathrm{NMR}\left(100 \mathrm{MHz}, \mathrm{CDCl}_{3}\right) \delta 166.9,146.9,136.4,129.5$, $128.4,128.3,102.0,64.3,41.7,35.9,31.4,28.3,25.6,22.5,14.1,2.1$; HRMS (ESI-TOF) $\mathrm{m} / \mathrm{z}:[\mathrm{M}+\mathrm{H}]^{+}$calcd for $\mathrm{C}_{33} \mathrm{H}_{43} \mathrm{INO}_{4}$ 644.2231, found 644.2232.

Diisopropyl 4-(2-iodoethyl)-2,6-diphenyl-1,4-dihydropyridine-3,5-dicarboxylate (2u). Eluent: $\mathrm{CH}_{2} \mathrm{Cl}_{2} / \mathrm{PE} \mathrm{67:33;} 5 \mathrm{~h}$; yield: $84 \mathrm{mg}, 60 \%$; white solid, mp $168-170{ }^{\circ} \mathrm{C}$; ${ }^{1} \mathrm{H}$ NMR $\left(400 \mathrm{MHz}, \mathrm{CDCl}_{3}\right) \delta 7.42-7.37(\mathrm{~m}, 6 \mathrm{H}), 7.37-7.33(\mathrm{~m}, 4 \mathrm{H}), 6.03(\mathrm{~s}, 1 \mathrm{H})$, 4.85 (hept, $J=6.4 \mathrm{~Hz}, 2 \mathrm{H}), 4.09(\mathrm{t}, J=6.0 \mathrm{~Hz}, 1 \mathrm{H}), 3.39-3.35(\mathrm{~m}, 2 \mathrm{H}), 2.34-2.28(\mathrm{~m}$, 2H), $0.99(\mathrm{~d}, J=6.4 \mathrm{~Hz}, 6 \mathrm{H}), 0.92(\mathrm{~d}, J=6.4 \mathrm{~Hz}, 6 \mathrm{H}) ;{ }^{13} \mathrm{C} \mathrm{NMR}\left(100 \mathrm{MHz}, \mathrm{CDCl}_{3}\right)$ $\delta 166.3,146.6,136.6,129.3,128.4,102.5,67.3,41.8,35.9,21.7,21.4,2.3$; HRMS (ESI-TOF) $\mathrm{m} / \mathrm{z}:[\mathrm{M}+\mathrm{H}]^{+}$calcd for $\mathrm{C}_{27} \mathrm{H}_{31} \mathrm{INO}_{4} 560.1292$, found 560.1292 .

Dicyclopentyl 4-(2-iodoethyl)-2,6-diphenyl-1,4-dihydropyridine-3,5-dicarboxylate (2v). Eluent: $\mathrm{CH}_{2} \mathrm{Cl}_{2} / \mathrm{PE}$ 67:33; $5 \mathrm{~h}$; yield: $98 \mathrm{mg}, 64 \%$; white solid, $\mathrm{mp}$ 147-149 ${ }^{\circ} \mathrm{C} ;{ }^{1} \mathrm{H}$ NMR $\left(400 \mathrm{MHz}, \mathrm{CDCl}_{3}\right) \delta 7.41-7.38(\mathrm{~m}, 6 \mathrm{H}), 7.35-7.32(\mathrm{~m}, 4 \mathrm{H})$, $6.02(\mathrm{~s}, 1 \mathrm{H}), 5.04(\mathrm{t}, J=6.0 \mathrm{~Hz}, 2 \mathrm{H}), 4.09(\mathrm{t}, J=6.0 \mathrm{~Hz}, 1 \mathrm{H}), 3.36(\mathrm{t}, J=8.8 \mathrm{~Hz}, 2 \mathrm{H})$, 2.33-2.27 (m, 2H), 1.66-1.59 (m, 4H), 1.41-1.31 (m, 12H); ${ }^{13} \mathrm{C}$ NMR (100 MHz, $\left.\mathrm{CDCl}_{3}\right) \delta 166.5,146.5,136.6,129.3,128.4,128.3,102.4,76.8,41.8,35.9,32.6,32.2$, 23.6, 23.5, 2.3; HRMS (ESI-TOF) $\mathrm{m} / \mathrm{z}:[\mathrm{M}+\mathrm{H}]^{+}$calcd for $\mathrm{C}_{31} \mathrm{H}_{35} \mathrm{INO}_{4}$ 612.1605, found 612.1606 . 
Dicyclohexyl 4-(2-iodoethyl)-2,6-diphenyl-1,4-dihydropyridine-3,5-dicarboxylate

(2w). Eluent: $\mathrm{CH}_{2} \mathrm{Cl}_{2} / \mathrm{PE} 67: 33$; 5 h; yield: $95 \mathrm{mg}$, 60\%; white solid, mp 151-153 ${ }^{\circ} \mathrm{C}$;

${ }^{1} \mathrm{H}$ NMR (400 MHz, $\left.\mathrm{CDCl}_{3}\right) \delta$ 7.41-7.34 (m, 10H), $6.05(\mathrm{~s}, 1 \mathrm{H}), 4.68-4.62(\mathrm{~m}, 2 \mathrm{H})$,

$4.13(\mathrm{t}, J=6.0 \mathrm{~Hz}, 1 \mathrm{H}), 3.39-3.35(\mathrm{~m}, 2 \mathrm{H}), 2.35-2.29(\mathrm{~m}, 2 \mathrm{H}), 1.67-1.41(\mathrm{~m}, 10 \mathrm{H})$, 1.26-1.06 (m, 10H); ${ }^{13} \mathrm{C} \mathrm{NMR}\left(100 \mathrm{MHz}, \mathrm{CDCl}_{3}\right) \delta 166.2,146.6,136.5,129.4$, $128.42,128.35,102.4,72.3,41.9,35.9,31.4,31.2,25.3,23.55,23.52,2.3$; HRMS (ESI-TOF) m/z: [M + H] $]^{+}$calcd for $\mathrm{C}_{33} \mathrm{H}_{39} \mathrm{INO}_{4}$ 640.1918, found 640.1920.

Di-tert-butyl 4-(2-iodoethyl)-2,6-diphenyl-1,4-dihydropyridine-3,5-dicarboxylate (2x). Eluent: $\mathrm{CH}_{2} \mathrm{Cl}_{2} / \mathrm{PE} 67: 33$; 5 h; yield: $45 \mathrm{mg}, 31 \%$; white solid, mp $144-147{ }^{\circ} \mathrm{C}$; ${ }^{1} \mathrm{H}$ NMR (400 MHz, $\left.\mathrm{CDCl}_{3}\right) \delta 7.41-7.39(\mathrm{~m}, 6 \mathrm{H}), 7.36-7.33(\mathrm{~m}, 4 \mathrm{H}), 5.97(\mathrm{~s}, 1 \mathrm{H})$, $3.93(\mathrm{t}, J=6.4 \mathrm{~Hz}, 1 \mathrm{H}), 3.40-3.36(\mathrm{~m}, 2 \mathrm{H}), 2.35-2.29(\mathrm{~m}, 2 \mathrm{H}), 1.15(\mathrm{~s}, 18 \mathrm{H}) ;{ }^{13} \mathrm{C}$ NMR (100 MHz, $\left.\mathrm{CDCl}_{3}\right) \delta 166.3,145.8,137.0,129.2,128.45,128.43,103.5,79.8$, 41.8, 36.4, 27.7, 2.7; HRMS (ESI-TOF) m/z: $[\mathrm{M}+\mathrm{Na}]^{+}$calcd for $\mathrm{C}_{2} \mathrm{H}_{34} \mathrm{INNaO}_{4}$ 610.1425 , found 610.1427

Dimethyl 2-(4-chlorophenyl)-4-(2-iodoethyl)-6-(p-tolyl)-1,4-dihydropyridine-3,5dicarboxylate (2be). The reaction was conducted according to the General Procedure for the Synthesis of 1,4-Dihydropyridines 2 using $0.25 \mathrm{mmol}$ of $\mathbf{1 b}$ and $0.25 \mathrm{mmol}$ of 1e. NMR of the crude products indicated that $\mathbf{2 b e}$ was formed as the major one with minor $\mathbf{2 b}$ and $\mathbf{2 e}$ formed as well. Pure $\mathbf{2 b e}$ was isolated through silica gel column chromatography, eluting with $\mathrm{CH}_{2} \mathrm{Cl}_{2} / \mathrm{PE}$ (67:33). 5 h; yield: $37 \mathrm{mg}, 27 \%$; white solid, mp $143-145{ }^{\circ} \mathrm{C} ;{ }^{1} \mathrm{H}$ NMR $\left(400 \mathrm{MHz}, \mathrm{CDCl}_{3}\right) \delta 7.39(\mathrm{dt}, J=8.4,2.0 \mathrm{~Hz}$, 2H), 7.31-7.23 (m, 6H, overlapped with the peak of chloroform), $6.07(\mathrm{~s}, 1 \mathrm{H}), 4.13(\mathrm{t}$, $J=6.4 \mathrm{~Hz}, 1 \mathrm{H}), 3.56(\mathrm{~d}, J=2.0 \mathrm{~Hz}, 6 \mathrm{H}), 3.34-3.29(\mathrm{~m}, 2 \mathrm{H}), 2.39$ (s, 3H), 2.30-2.24 $(\mathrm{m}, 2 \mathrm{H}) ;{ }^{13} \mathrm{C} \mathrm{NMR}\left(100 \mathrm{MHz}, \mathrm{CDCl}_{3}\right) \delta 167.1,166.9,147.4,146.2,139.9,135.7$, $134.4,132.8,129.7,129.2,128.8,128.1,102.1,101.5,51.34,51.29,41.4,35.6,21.4$, 
1.7; HRMS (ESI-TOF) m/z: $[\mathrm{M}+\mathrm{H}]^{+}$calcd for $\mathrm{C}_{24} \mathrm{H}_{24} \mathrm{ClINO}_{4}$ 552.0433, found 552.0432.

4. Procedure for the Gram-Scale Synthesis of 2a. A solution of the enamine substrate (1a) (1.3 g, $6 \mathrm{mmol})$ in DCE $(120 \mathrm{~mL})$ was treated sequentially with iodine (1.83 g, $7.2 \mathrm{mmol})$ and $\mathrm{NH}_{4} \mathrm{PF}_{6}(2.93 \mathrm{~g}, 18 \mathrm{mmol})$. The reaction mixture was stirred at $0{ }^{\circ} \mathrm{C}$ for $1 \mathrm{~h}$, and then at room temperature $\left(25^{\circ} \mathrm{C}\right)$ for $4 \mathrm{~h}(\mathrm{TLC}$ indicated the completion of the reaction). It was quenched with sat. $\mathrm{Na}_{2} \mathrm{~S}_{2} \mathrm{O}_{3}(24 \mathrm{~mL})$, diluted with water $(120 \mathrm{~mL})$, and extracted with $\mathrm{CH}_{2} \mathrm{Cl}_{2}(120 \mathrm{~mL} \times 3)$. The combined organic layer was dried over anhydrous $\mathrm{Na}_{2} \mathrm{SO}_{4}$, concentrated and purified through silica gel column chromatography, eluting with $\mathrm{CH}_{2} \mathrm{Cl}_{2} / \mathrm{PE}$ (67:33) to afford 2a as white solid (yield: $1.1 \mathrm{~g}, 72 \%$ ).

5. Isolation and Characterization of Intermediate $\mathbf{J}$. According to the General Procedure for the Synthesis of 1,4-Dihydropyridines 2, the reaction was conducted using $0.5 \mathrm{mmol}$ of $\mathbf{1 a}$. After stirred at $0{ }^{\circ} \mathrm{C}$ for $1 \mathrm{~h}$, it was directly quenched with sat. $\mathrm{Na}_{2} \mathrm{~S}_{2} \mathrm{O}_{3}(2 \mathrm{~mL})$, diluted with water $(10 \mathrm{~mL})$, and extracted with $\mathrm{CH}_{2} \mathrm{Cl}_{2}(10 \mathrm{~mL} \times 3)$. The combined organic layer was dried over anhydrous $\mathrm{Na}_{2} \mathrm{SO}_{4}$, concentrated and purified through silica gel column chromatography, eluting with $\mathrm{CH}_{2} \mathrm{Cl}_{2} / \mathrm{PE}$ (67:33) to give a less stable intermediate $\mathbf{J}$ as white solid (yield: $89 \mathrm{mg}, 71 \%$ ). ${ }^{1} \mathrm{H}$ NMR (400 $\left.\mathrm{MHz}, \mathrm{CDCl}_{3}\right) \delta 8.07-8.04(\mathrm{~m}, 2 \mathrm{H}), 7.59-7.56(\mathrm{~m}, 2 \mathrm{H}), 7.52-7.46(\mathrm{~m}, 3 \mathrm{H}), 7.40-7.37$ (m, 3H), $4.09(\mathrm{~d}, J=1.2 \mathrm{~Hz}, 1 \mathrm{H}), 3.81-3.77(\mathrm{~m}, 1 \mathrm{H}), 3.64(\mathrm{~s}, 3 \mathrm{H}), 3.61(\mathrm{~s}, 3 \mathrm{H})$, 3.19-3.12 (m, 1H), 3.07-3.01 (m, 1H), 2.07-1.97 (m, 1H), $1.87-1.78(\mathrm{~m}, 1 \mathrm{H}) ;{ }^{13} \mathrm{C}$ NMR $\left(100 \mathrm{MHz}, \mathrm{CDCl}_{3}\right) \delta 169.1,168.5,160.3,153.5,137.8,136.9,131.7,129.0$, $128.8,127.9,127.8,114.4,52.9,51.9,42.6,37.1,35.1,1.3$. 
6. Capture of Cyclopropylamine Formed during the Synthesis of 2a. According to the General procedure for the Synthesis of 1,4-Dihydropyridines 2, upon the complete consumption of the enamine substrate $1 \mathrm{a}(0.5 \mathrm{mmol})$, the reaction mixture was further treated with triethylamine $(63 \mathrm{mg}, 0.625 \mathrm{mmol})$, and then benzoyl chloride (70 $\mathrm{mg}$, $0.5 \mathrm{mmol})$ in $\mathrm{CH}_{2} \mathrm{Cl}_{2}(0.5 \mathrm{~mL})$ slowly at $0{ }^{\circ} \mathrm{C}$. After stirred for $16 \mathrm{~h}$, it was quenched with $1 \mathrm{~N} \mathrm{HCl}(1.5 \mathrm{~mL})$ and sat. $\mathrm{Na}_{2} \mathrm{~S}_{2} \mathrm{O}_{3}(2 \mathrm{~mL})$, diluted with water $(6 \mathrm{~mL})$, and extracted with $\mathrm{CH}_{2} \mathrm{Cl}_{2}(10 \mathrm{~mL} \times 3)$. The combined organic layer was washed with 1 $\mathrm{M} \mathrm{NaOH}(30 \mathrm{~mL})$ and brine $(30 \mathrm{~mL})$ in sequence, dried over anhydrous $\mathrm{Na}_{2} \mathrm{SO}_{4}$, concentrated and purified through silica gel column chromatography, eluting with EtOAc/PE (20:80) to give $N$-cyclopropylbenzamide (K) as white solid (yield: 33mg,

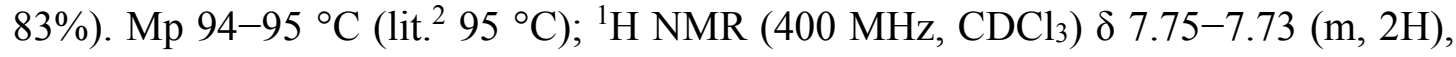
7.50-7.46 (m, 1H), 7.42-7.39 (m, 2H), $6.41(\mathrm{~s}, 1 \mathrm{H}), 2.93-2.87(\mathrm{~m}, 1 \mathrm{H}), 0.88-0.84$ $(\mathrm{m}, 2 \mathrm{H}), 0.64-0.60(\mathrm{~m}, 2 \mathrm{H}) ;{ }^{13} \mathrm{C} \mathrm{NMR}\left(100 \mathrm{MHz}, \mathrm{CDCl}_{3}\right) \delta$ 169.0, 134.4, 131.5, $128.5,126.8,23.1,6.8$.

7. General Procedure for the Synthesis of Pyridines 3. This reaction was conducted according to the General procedure for the Synthesis of 1,4-Dihydropyridines 2, and upon the complete consumption of the enamine substrate $1(0.5 \mathrm{mmol})$, the reaction mixture was further treated with 2,3-dichloro-5,6-dicyano-1,4-benzoquinone (DDQ, $136 \mathrm{mg}, 0.6 \mathrm{mmol})$. After being stirred for $1 \mathrm{~h}$, it was quenched with sat. $\mathrm{NaHCO}_{3}(4$ $\mathrm{mL})$ and sat. $\mathrm{Na}_{2} \mathrm{~S}_{2} \mathrm{O}_{3}(2 \mathrm{~mL})$, diluted with water $(6 \mathrm{~mL})$, and extracted with $\mathrm{CH}_{2} \mathrm{Cl}_{2}$ $(10 \mathrm{~mL} \times 3)$. The combined organic layer was dried over anhydrous $\mathrm{Na}_{2} \mathrm{SO}_{4}$, concentrated and purified by silica gel column chromatography to afford the pyridine product (3). 
Dimethyl 4-(2-iodoethyl)-2,6-diphenylpyridine-3,5-dicarboxylate (3a). Eluent: $\mathrm{CH}_{2} \mathrm{Cl}_{2} / \mathrm{PE} \mathrm{50:50;} 6 \mathrm{~h}$; yield: $95 \mathrm{mg}, 76 \%$; white solid, mp $132-134{ }^{\circ} \mathrm{C}$; ${ }^{1} \mathrm{H}$ NMR (400 MHz, $\left.\mathrm{CDCl}_{3}\right) \delta$ 7.67-7.65 (m, 4H), 7.46-7.43 (m, 6H), $3.72(\mathrm{~s}, 6 \mathrm{H}), 3.49-3.45$ $(\mathrm{m}, 2 \mathrm{H}), 3.28-3.23(\mathrm{~m}, 2 \mathrm{H}) ;{ }^{13} \mathrm{C} \mathrm{NMR}\left(100 \mathrm{MHz}, \mathrm{CDCl}_{3}\right) \delta 168.6,157.4,146.4$, 139.2, 129.3, 128.5, 128.3, 126.7, 52.9, 36.9, 0.2; HRMS (ESI-TOF) m/z: $[\mathrm{M}+\mathrm{H}]^{+}$ calcd for $\mathrm{C}_{23} \mathrm{H}_{21} \mathrm{INO}_{4}$ 502.0510, found 502.0512.

Dimethyl 4-(2-iodoethyl)-2,6-di-p-tolylpyridine-3,5-dicarboxylate (3b). Eluent: $\mathrm{CH}_{2} \mathrm{Cl}_{2} / \mathrm{PE} \mathrm{50:50;} 6 \mathrm{~h}$; yield: $91 \mathrm{mg}, 69 \%$; white solid, mp $138-140{ }^{\circ} \mathrm{C}$; ${ }^{1} \mathrm{H}$ NMR $\left(400 \mathrm{MHz}, \mathrm{CDCl}_{3}\right) \delta 7.56(\mathrm{~d}, J=8.0 \mathrm{~Hz}, 4 \mathrm{H}), 7.24(\mathrm{~d}, J=8.0 \mathrm{~Hz}, 4 \mathrm{H}$, overlapped with the peak of chloroform), $3.74(\mathrm{~s}, 6 \mathrm{H}), 3.47-3.43(\mathrm{~m}, 2 \mathrm{H}), 3.25-3.21(\mathrm{~m}, 2 \mathrm{H})$, $2.39(\mathrm{~s}, 6 \mathrm{H}) ;{ }^{13} \mathrm{C} \mathrm{NMR}\left(100 \mathrm{MHz}, \mathrm{CDCl}_{3}\right) \delta 168.8,157.3,146.1,139.3,136.4,129.2$, 128.2, 126.2, 52.8, 37.0, 21.3, 0.3; HRMS (ESI-TOF) m/z: $[\mathrm{M}+\mathrm{H}]^{+}$calcd for $\mathrm{C}_{25} \mathrm{H}_{25} \mathrm{INO}_{4} 530.0823$, found 530.0824 .

Dimethyl 4-(2-iodoethyl)-2,6-bis(4-methoxyphenyl)pyridine-3,5-dicarboxylate (3c). Eluent: $\mathrm{CH}_{2} \mathrm{Cl}_{2} / \mathrm{PE} \mathrm{50:50;} 8$ h; yield: $74 \mathrm{mg}$, 53\%; white solid, mp 130-132 ${ }^{\circ} \mathrm{C}$; ${ }^{1} \mathrm{H}$ NMR $\left(400 \mathrm{MHz}, \mathrm{CDCl}_{3}\right) \delta 7.63(\mathrm{dt}, J=10.0,3.2 \mathrm{~Hz}, 4 \mathrm{H}), 6.96(\mathrm{dt}, J=9.2,2.8 \mathrm{~Hz}$, 4H), $3.85(\mathrm{~s}, 6 \mathrm{H}), 3.76(\mathrm{~s}, 6 \mathrm{H}), 3.47-3.43(\mathrm{~m}, 2 \mathrm{H}), 3.24-3.20(\mathrm{~m}, 2 \mathrm{H}) ;{ }^{13} \mathrm{C}$ NMR $(100$ $\left.\mathrm{MHz}, \mathrm{CDCl}_{3}\right) \delta 169.0,160.6,156.7,146.2,131.7,129.8,125.7,113.9,55.3,52.9$, 37.0, 0.4; HRMS (ESI-TOF) m/z: $[\mathrm{M}+\mathrm{H}]^{+}$calcd for $\mathrm{C}_{25} \mathrm{H}_{25} \mathrm{INO}_{6} 562.0721$, found 562.0723.

Dimethyl 2,6-bis(4-fluorophenyl)-4-(2-iodoethyl)pyridine-3,5-dicarboxylate (3d). Eluent: $\mathrm{CH}_{2} \mathrm{Cl}_{2} / \mathrm{PE}$ 50:50; $6 \mathrm{~h}$; yield: $81 \mathrm{mg}, 60 \%$; white solid, mp $129-131{ }^{\circ} \mathrm{C}$; ${ }^{1} \mathrm{H}$ NMR (400 MHz, $\left.\mathrm{CDCl}_{3}\right) \delta 7.64(\mathrm{dd}, J=7.6,5.6 \mathrm{~Hz}, 4 \mathrm{H}), 7.14(\mathrm{t}, J=8.4 \mathrm{~Hz}, 4 \mathrm{H})$, $3.75(\mathrm{~s}, 6 \mathrm{H}), 3.46-3.42(\mathrm{~m}, 2 \mathrm{H}), 3.26-3.22(\mathrm{~m}, 2 \mathrm{H}) ;{ }^{13} \mathrm{C} \mathrm{NMR}\left(100 \mathrm{MHz}, \mathrm{CDCl}_{3}\right) \delta$ $168.4,163.5\left(\mathrm{~d}, J_{\mathrm{C}-\mathrm{F}}=248.2 \mathrm{~Hz}\right), 156.3,146.6,135.2\left(\mathrm{~d}, J_{\mathrm{C}-\mathrm{F}}=3.2 \mathrm{~Hz}\right), 130.3(\mathrm{~d}$, 
$\left.J_{\mathrm{C}-\mathrm{F}}=8.5 \mathrm{~Hz}\right), 126.7,115.6\left(\mathrm{~d}, J_{\mathrm{C}-\mathrm{F}}=21.6 \mathrm{~Hz}\right), 53.0,36.9,-0.1 ;{ }^{19} \mathrm{~F} \mathrm{NMR}(376 \mathrm{MHz}$, $\left.\mathrm{CDCl}_{3}\right) \delta$-111.6; HRMS (ESI-TOF) m/z: $[\mathrm{M}+\mathrm{H}]^{+}$calcd for $\mathrm{C}_{23} \mathrm{H}_{19} \mathrm{~F}_{2} \mathrm{INO}_{4}$ 538.0321, found 538.0324 .

Dimethyl 2,6-bis(4-chlorophenyl)-4-(2-iodoethyl)pyridine-3,5-dicarboxylate (3e). Eluent: $\mathrm{CH}_{2} \mathrm{Cl}_{2} / \mathrm{PE}$ 50:50; $7 \mathrm{~h}$; yield: $87 \mathrm{mg}, 61 \%$; white solid, mp $150-152{ }^{\circ} \mathrm{C}$; ${ }^{1} \mathrm{H}$ NMR (400 MHz, $\left.\mathrm{CDCl}_{3}\right) \delta 7.59(\mathrm{dt}, J=9.2,2.4 \mathrm{~Hz}, 4 \mathrm{H}), 7.43(\mathrm{dt}, J=9.2,2.4 \mathrm{~Hz}$, 4H), $3.75(\mathrm{~s}, 6 \mathrm{H}), 3.45-3.41(\mathrm{~m}, 2 \mathrm{H}), 3.27-3.23(\mathrm{~m}, 2 \mathrm{H}) ;{ }^{13} \mathrm{C}$ NMR (100 MHz, $\left.\mathrm{CDCl}_{3}\right) \delta 168.2,156.2,146.8,137.4,135.8,129.7,128.8,126.9,53.1,36.8,-0.2$; HRMS (ESI-TOF) m/z: $[\mathrm{M}+\mathrm{H}]^{+}$calcd for $\mathrm{C}_{23} \mathrm{H}_{19} \mathrm{Cl}_{2} \mathrm{INO}_{4}$ 569.9730, found 569.9732.

Dimethyl 2,6-bis(4-bromophenyl)-4-(2-iodoethyl)pyridine-3,5-dicarboxylate (3f). Eluent: $\mathrm{CH}_{2} \mathrm{Cl}_{2} / \mathrm{PE}$ 50:50; $7 \mathrm{~h}$; yield: $98 \mathrm{mg}, 60 \%$; white solid, mp $175-177{ }^{\circ} \mathrm{C}$; ${ }^{1} \mathrm{H}$ NMR (400 MHz, $\left.\mathrm{CDCl}_{3}\right) \delta 7.59(\mathrm{dt}, J=8.8,2.4 \mathrm{~Hz}, 4 \mathrm{H}), 7.52(\mathrm{dt}, J=8.8,2.4 \mathrm{~Hz}$, 4H), $3.75(\mathrm{~s}, 6 \mathrm{H}), 3.45-3.40(\mathrm{~m}, 2 \mathrm{H}), 3.27-3.23(\mathrm{~m}, 2 \mathrm{H}) ;{ }^{13} \mathrm{C}$ NMR (100 MHz, $\left.\mathrm{CDCl}_{3}\right) \delta 168.2,156.3,146.8,137.8,131.7,129.9,126.9,124.1,53.1,36.8,-0.2$; HRMS (ESI-TOF) m/z: $[\mathrm{M}+\mathrm{H}]^{+}$calcd for $\mathrm{C}_{23} \mathrm{H}_{19} \mathrm{Br}_{2} \mathrm{INO}_{4}$ 657.8720, found 657.8720.

Dimethyl 4-(2-iodoethyl)-2,6-di-m-tolylpyridine-3,5-dicarboxylate (3i). Eluent: $\mathrm{CH}_{2} \mathrm{Cl}_{2} / \mathrm{PE}$ 50:50; $7 \mathrm{~h}$; yield: $95 \mathrm{mg}, 72 \%$; white solid, mp $126-128{ }^{\circ} \mathrm{C} ;{ }^{1} \mathrm{H} \mathrm{NMR}$ $\left(400 \mathrm{MHz}, \mathrm{CDCl}_{3}\right) \delta 7.50(\mathrm{~s}, 2 \mathrm{H}), 7.41(\mathrm{~d}, J=7.6 \mathrm{~Hz}, 2 \mathrm{H}), 7.32(\mathrm{t}, J=7.6 \mathrm{~Hz}, 2 \mathrm{H})$, $7.24(\mathrm{~d}, J=7.6 \mathrm{~Hz}, 2 \mathrm{H}$, overlapped with the peak of chloroform), $3.72(\mathrm{~s}, 6 \mathrm{H})$, 3.49-3.44 (m, 2H), 3.26-3.22 (m, 2H), $\left.2.41(\mathrm{~s}, 6 \mathrm{H}) ;{ }^{13} \mathrm{C} \mathrm{NMR} \mathrm{(100} \mathrm{MHz,} \mathrm{CDCl}_{3}\right) \delta$ $168.6,157.6,146.2,139.1,138.3,130.1,129.1,128.3,126.7,125.3,52.8,37.0,21.5$, 0.3; HRMS (ESI-TOF) m/z: $[\mathrm{M}+\mathrm{H}]^{+}$calcd for $\mathrm{C}_{25} \mathrm{H}_{25} \mathrm{INO}_{4}$ 530.0823, found 530.0826. 
Dimethyl 2,6-bis(2-fluorophenyl)-4-(2-iodoethyl)pyridine-3,5-dicarboxylate (3j). Eluent: $\mathrm{CH}_{2} \mathrm{Cl}_{2} / \mathrm{PE} \mathrm{50:50;} 7 \mathrm{~h}$; yield: $94 \mathrm{mg}, 70 \%$; white solid, mp 92-94 ${ }^{\circ} \mathrm{C} ;{ }^{1} \mathrm{H}$ NMR (400 MHz, $\left.\mathrm{CDCl}_{3}\right) \delta 7.55(\mathrm{td}, J=7.6,2.0 \mathrm{~Hz}, 2 \mathrm{H}), 7.44-7.38(\mathrm{~m}, 2 \mathrm{H}), 7.24(\mathrm{td}$, $J=7.6,1.2 \mathrm{~Hz}, 2 \mathrm{H}$, overlapped with the peak of chloroform), 7.13 (ddd, $J=10.4,8.4$, $1.2 \mathrm{~Hz}, 2 \mathrm{H}), 3.73(\mathrm{~s}, 6 \mathrm{H}), 3.57-3.53(\mathrm{~m}, 2 \mathrm{H}), 3.35-3.31(\mathrm{~m}, 2 \mathrm{H}) ;{ }^{13} \mathrm{C}$ NMR $(100$ $\left.\mathrm{MHz}, \mathrm{CDCl}_{3}\right) \delta 167.2,159.6(\mathrm{~d}, J=247.1 \mathrm{~Hz}), 154.0,147.0,131.2,131.1\left(\mathrm{~d}, J_{\mathrm{C}-\mathrm{F}}=\right.$ 11.2 Hz), 128.7, $127.0\left(\mathrm{~d}, J_{\mathrm{C}-\mathrm{F}}=14.6 \mathrm{~Hz}\right), 124.4\left(\mathrm{~d}, J_{\mathrm{C}-\mathrm{F}}=3.5 \mathrm{~Hz}\right), 115.6\left(\mathrm{~d}, J_{\mathrm{C}-\mathrm{F}}=\right.$ 21.6 Hz), 52.8, 37.4, 0.7; ${ }^{19} \mathrm{~F}$ NMR (376 MHz, $\left.\mathrm{CDCl}_{3}\right) \delta-116.2$; HRMS (ESI-TOF) $\mathrm{m} / \mathrm{z}:[\mathrm{M}+\mathrm{H}]^{+}$calcd for $\mathrm{C}_{23} \mathrm{H}_{19} \mathrm{~F}_{2} \mathrm{INO}_{4}$ 538.0321, found 538.0323.

Dimethyl 4-(2-iodoethyl)-2,6-di(naphthalen-2-yl)pyridine-3,5-dicarboxylate (3m). Eluent: $\mathrm{CH}_{2} \mathrm{Cl}_{2} / \mathrm{PE}$ 50:50; 6 h; yield: $92 \mathrm{mg}, 61 \%$; white solid, mp $187-189{ }^{\circ} \mathrm{C}$; ${ }^{1} \mathrm{H}$ NMR (400 MHz, $\left.\mathrm{CDCl}_{3}\right) \delta 8.19(\mathrm{~s}, 2 \mathrm{H}), 7.95-7.87(\mathrm{~m}, 6 \mathrm{H}), 7.83(\mathrm{~d}, J=8.4 \mathrm{~Hz}, 2 \mathrm{H})$, 7.54-7.52 (m, 4H), $3.69(\mathrm{~s}, 6 \mathrm{H}), 3.55-3.50(\mathrm{~m}, 2 \mathrm{H}), 3.35-3.31(\mathrm{~m}, 2 \mathrm{H}) ;{ }^{13} \mathrm{C} \mathrm{NMR}$ $\left(100 \mathrm{MHz}, \mathrm{CDCl}_{3}\right) \delta 168.7,157.4,146.6,136.5,133.5,133.1,128.6,128.3,128.2$, 127.7, 127.0, 126.9, 126.5, 125.7, 53.0, 36.9, 0.2; HRMS (ESI-TOF) m/z: $[\mathrm{M}+\mathrm{H}]^{+}$ calcd for $\mathrm{C}_{31} \mathrm{H}_{25} \mathrm{INO}_{4} 602.0823$, found 602.0827 .

Dibenzyl 4-(2-iodoethyl)-2,6-diphenylpyridine-3,5-dicarboxylate (3o). Eluent: $\mathrm{CH}_{2} \mathrm{Cl}_{2} / \mathrm{PE}$ 50:50; $5 \mathrm{~h}$; yield: $100 \mathrm{mg}, 61 \%$; white solid, mp $126-128{ }^{\circ} \mathrm{C} ;{ }^{1} \mathrm{H} \mathrm{NMR}$ $\left(400 \mathrm{MHz} \mathrm{CDCl}_{3}\right) \delta 7.62-7.60(\mathrm{~m}, 4 \mathrm{H}), 7.42-7.35(\mathrm{~m}, 6 \mathrm{H}), 7.30-7.25(\mathrm{~m}, 6 \mathrm{H}$, overlapped with the peak of chloroform), 7.04-7.02 (m, 4H), 5.11 (s, 4H), 3.41-3.37 (m, 2H), 3.25-3.21 (m, 2H); ${ }^{13} \mathrm{C}$ NMR (100 MHz, $\left.\mathrm{CDCl}_{3}\right) \delta 167.8,157.5,146.4$, $139.2,134.2,129.3,128.8,128.61,128.60,128.5,128.4,126.7,68.1,36.7,0.3$; HRMS (ESI-TOF) m/z: $[\mathrm{M}+\mathrm{H}]^{+}$calcd for $\mathrm{C}_{35} \mathrm{H}_{29} \mathrm{INO}_{4}$ 654.1136, found 654.1135.

Diethyl 4-(2-iodoethyl)-2,6-diphenylpyridine-3,5-dicarboxylate (3s). Eluent: $\mathrm{CH}_{2} \mathrm{Cl}_{2} / \mathrm{PE}$ 50:50; 6 h; yield: $98 \mathrm{mg}, 74 \%$; white solid, mp $84-86{ }^{\circ} \mathrm{C} ;{ }^{1} \mathrm{H}$ NMR (400 
$\left.\mathrm{MHz}, \mathrm{CDCl}_{3}\right) \delta 7.66-7.63(\mathrm{~m}, 4 \mathrm{H}), 7.44-7.42(\mathrm{~m}, 6 \mathrm{H}), 4.19(\mathrm{q}, J=7.2 \mathrm{~Hz}, 4 \mathrm{H})$, 3.51-3.47 (m, 2H), 3.30-3.25 (m, 2H), $1.07(\mathrm{t}, J=7.2 \mathrm{~Hz}, 6 \mathrm{H}) ;{ }^{13} \mathrm{C}$ NMR $(100 \mathrm{MHz}$, $\left.\mathrm{CDCl}_{3}\right) \delta 168.0,157.5,146.1,139.3,129.2,128.42,128.40,127.1,62.1,36.9,13.7$, 0.4; HRMS (ESI-TOF) m/z: $[\mathrm{M}+\mathrm{H}]^{+}$calcd for $\mathrm{C}_{25} \mathrm{H}_{25} \mathrm{INO}_{4}$ 530.0823, found 530.0825 .

8. General Procedure for the Synthesis of Compounds 4 and 5 from 2. A mixture containing a 1,4-dihydropyridine $2(0.2 \mathrm{mmol})$ and $\mathrm{K}_{2} \mathrm{CO}_{3}(53 \mathrm{mg}, 0.4 \mathrm{mmol})$ in DMF $(2 \mathrm{~mL})$ was stirred at room temperature until TLC indicated the complete consumption of 2 . The reaction was then quenched with water $(10 \mathrm{~mL})$ and extracted with EtOAc $(10 \mathrm{~mL} \times 3)$. The combined organic layer was dried over anhydrous $\mathrm{Na}_{2} \mathrm{SO}_{4}$, concentrated and purified through silica gel column chromatography to give products $\mathbf{4}$ and $\mathbf{5}$.

\section{Dimethyl (1s,4s)-2,6-diphenyl-1-azabicyclo[2.2.2]octa-2,5-diene-3,5-dicarboxylate} (4a). Eluent: $\mathrm{CH}_{2} \mathrm{Cl}_{2} / \mathrm{PE} 67: 33 ; 2$ h; yield: $28 \mathrm{mg}, 37 \%$; white solid, mp $110-112{ }^{\circ} \mathrm{C}$; ${ }^{1} \mathrm{H}$ NMR $\left(400 \mathrm{MHz}, \mathrm{CDCl}_{3}\right) \delta 7.61-7.59(\mathrm{~m}, 4 \mathrm{H}), 7.36-7.33(\mathrm{~m}, 6 \mathrm{H}), 4.70(\mathrm{t}, J=2.8$ $\mathrm{Hz}, 1 \mathrm{H}), 3.72(\mathrm{~s}, 6 \mathrm{H}), 3.03-3.00(\mathrm{~m}, 2 \mathrm{H}), 2.00-1.96(\mathrm{~m}, 2 \mathrm{H}) ;{ }^{13} \mathrm{C}$ NMR $(100 \mathrm{MHz}$, $\left.\mathrm{CDCl}_{3}\right) \delta 166.2,159.7,135.6,135.0,129.2,129.0,127.7,51.7,49.7,39.8,29.2$ HRMS (ESI-TOF) m/z: [M + H] $]^{+}$calcd for $\mathrm{C}_{23} \mathrm{H}_{22} \mathrm{NO}_{4} 376.1543$, found 376.1553 .

\section{Dimethyl}

$(1 s, 4 s)-2,6-d i-p$-tolyl-1-azabicyclo[2.2.2]octa-2,5-diene-3,5dicarboxylate (4b). Eluent: $\mathrm{CH}_{2} \mathrm{Cl}_{2} / \mathrm{PE} 67: 33 ; 4$ h; yield: $19 \mathrm{mg}, 24 \%$; white solid, mp $107-108{ }^{\circ} \mathrm{C} ;{ }^{1} \mathrm{H}$ NMR $\left(400 \mathrm{MHz}, \mathrm{CDCl}_{3}\right) \delta 7.52(\mathrm{~d}, J=8.4 \mathrm{~Hz}, 4 \mathrm{H}), 7.15(\mathrm{~d}, J=$ $8.0 \mathrm{~Hz}, 4 \mathrm{H}), 4.67(\mathrm{t}, J=2.4 \mathrm{~Hz}, 1 \mathrm{H}), 3.72(\mathrm{~s}, 6 \mathrm{H}), 2.97(\mathrm{t}, J=6.8 \mathrm{~Hz}, 2 \mathrm{H}), 2.36(\mathrm{~s}$, 6H), 1.97-1.93 (m, 2H); ${ }^{13} \mathrm{C}$ NMR (100 MHz, $\left.\mathrm{CDCl}_{3}\right) \delta 166.4,159.9,139.4,134.8$, 
132.2, 129.1, 128.5, 51.7, 49.7, 39.8, 29.3, 21.4; HRMS (ESI-TOF) m/z: $[\mathrm{M}+\mathrm{H}]^{+}$ calcd for $\mathrm{C}_{25} \mathrm{H}_{26} \mathrm{NO}_{4} 404.1856$, found 404.1865 .

Dimethyl (1s,4s)-2,6-bis(4-bromophenyl)-1-azabicyclo[2.2.2]octa-2,5-diene-3,5dicarboxylate (4f). Eluent: $\mathrm{CH}_{2} \mathrm{Cl}_{2} / \mathrm{PE} 67: 33$; 4 h; yield: $45 \mathrm{mg}, 42 \%$; white solid, mp $125-126{ }^{\circ} \mathrm{C} ;{ }^{1} \mathrm{H}$ NMR $\left(400 \mathrm{MHz}, \mathrm{CDCl}_{3}\right) \delta 7.47(\mathrm{~s}, 8 \mathrm{H}), 4.71(\mathrm{t}, J=2.8 \mathrm{~Hz}, 1 \mathrm{H})$, $3.74(\mathrm{~s}, 6 \mathrm{H}), 2.99-2.96(\mathrm{~m}, 2 \mathrm{H}), 2.00-1.96(\mathrm{~m}, 2 \mathrm{H}) ;{ }^{13} \mathrm{C} \mathrm{NMR}\left(100 \mathrm{MHz}, \mathrm{CDCl}_{3}\right) \delta$ $165.7,158.6,136.1,133.7,131.0,130.6,123.8,51.9,49.8,39.9,29.3$; HRMS (ESI-TOF) m/z: $[\mathrm{M}+\mathrm{H}]^{+}$calcd for $\mathrm{C}_{23} \mathrm{H}_{20} \mathrm{Br}_{2} \mathrm{NO}_{4}$ 531,9754, found 531.9749 .

Diethyl $(1 s, 4 s)-2,6$-diphenyl-1-azabicyclo[2.2.2]octa-2,5-diene-3,5-dicarboxylate (4s). Eluent: $\mathrm{CH}_{2} \mathrm{Cl}_{2} / \mathrm{PE} 67: 33 ; 10 \mathrm{~h}$; yield: $18 \mathrm{mg}, 22 \%$; white solid, mp 94-96 ${ }^{\circ} \mathrm{C}$; ${ }^{1} \mathrm{H}$ NMR $\left(400 \mathrm{MHz}, \mathrm{CDCl}_{3}\right) \delta 7.61-7.59(\mathrm{~m}, 4 \mathrm{H}), 7.35-7.33(\mathrm{~m}, 6 \mathrm{H}), 4.70(\mathrm{~s}, 1 \mathrm{H})$, 4.25-4.12 (m, 4H), $3.01(\mathrm{t}, J=6.8 \mathrm{~Hz}, 2 \mathrm{H}), 1.98(\mathrm{t}, J=5.2 \mathrm{~Hz}, 2 \mathrm{H}), 1.19(\mathrm{t}, J=7.2$ $\mathrm{Hz}, 6 \mathrm{H}) ;{ }^{13} \mathrm{C} \mathrm{NMR}\left(100 \mathrm{MHz}, \mathrm{CDCl}_{3}\right) \delta 165.9,159.4,136.1,135.2,129.12,129.09$, 127.7, 60.7, 49.7, 39.8, 29.1, 13.9; HRMS (ESI-TOF) m/z: $[\mathrm{M}+\mathrm{H}]^{+}$calcd for $\mathrm{C}_{25} \mathrm{H}_{26} \mathrm{NO}_{4} 404.1856$, found 404.1855 .

Dimethyl 2,6-diphenylpyridine-3,5-dicarboxylate (5a). Eluent: $\mathrm{CH}_{2} \mathrm{Cl}_{2} / \mathrm{PE} 67: 33 ; 2$ h; yield: $35 \mathrm{mg}, 51 \%$; white solid, mp $108-110{ }^{\circ} \mathrm{C} ;{ }^{1} \mathrm{H}$ NMR (400 $\left.\mathrm{MHz}, \mathrm{CDCl}_{3}\right) \delta$ $8.56(\mathrm{~s}, 1 \mathrm{H}), 7.66-7.63(\mathrm{~m}, 4 \mathrm{H}), 7.46-7.44(\mathrm{~m}, 6 \mathrm{H}), 3.75(\mathrm{~s}, 6 \mathrm{H}) ;{ }^{13} \mathrm{C}$ NMR $(100$ $\left.\mathrm{MHz}, \mathrm{CDCl}_{3}\right) \delta 167.8,159.8,140.6,139.1,129.4,128.9,128.2,124.3,52.5 ;$ HRMS (ESI-TOF) m/z: $[\mathrm{M}+\mathrm{H}]^{+}$calcd for $\mathrm{C}_{21} \mathrm{H}_{18} \mathrm{NO}_{4} 348.1230$, found 348.1236.

Dimethyl 2,6-di-p-tolylpyridine-3,5-dicarboxylate (5b). Eluent: $\mathrm{CH}_{2} \mathrm{Cl}_{2} / \mathrm{PE}$ 67:33; $4 \mathrm{~h}$; yield: $26 \mathrm{mg}, 35 \%$; white solid, mp $122-124{ }^{\circ} \mathrm{C} ;{ }^{1} \mathrm{H}$ NMR (400 MHz, $\left.\mathrm{CDCl}_{3}\right) \delta$ $8.52(\mathrm{~s}, 1 \mathrm{H}), 7.55(\mathrm{~d}, J=8.0 \mathrm{~Hz}, 4 \mathrm{H}), 7.25(\mathrm{~d}, J=7.6 \mathrm{~Hz}, 4 \mathrm{H}$, overlapped with the peak of chloroform), $3.77(\mathrm{~s}, 6 \mathrm{H}), 2.41(\mathrm{~s}, 6 \mathrm{H}) ;{ }^{13} \mathrm{C} \mathrm{NMR}\left(100 \mathrm{MHz}, \mathrm{CDCl}_{3}\right) \delta 168.0$, 
159.7, 140.6, 139.5, 136.3, 129.0, 128.9, 123.6, 52.5, 21.4; HRMS (ESI-TOF) m/z: $[\mathrm{M}+\mathrm{H}]^{+}$calcd for $\mathrm{C}_{23} \mathrm{H}_{22} \mathrm{NO}_{4} 376.1543$, found 376.1548 .

Dimethyl 2,6-bis(4-bromophenyl)pyridine-3,5-dicarboxylate (5f). Eluent: $\mathrm{CH}_{2} \mathrm{Cl}_{2} / \mathrm{PE} 67: 33$; $4 \mathrm{~h}$; yield: $58 \mathrm{mg}, 57 \%$; white solid, mp $125-126{ }^{\circ} \mathrm{C}$; ${ }^{1} \mathrm{H}$ NMR $\left(400 \mathrm{MHz}, \mathrm{CDCl}_{3}\right) \delta 8.60(\mathrm{~s}, 1 \mathrm{H}), 7.59(\mathrm{dt}, J=8.8,2.4 \mathrm{~Hz}, 4 \mathrm{H}), 7.50(\mathrm{dt}, J=8.8,2.4$ $\mathrm{Hz}, 4 \mathrm{H}), 3.79(\mathrm{~s}, 6 \mathrm{H}) ;{ }^{13} \mathrm{C} \mathrm{NMR}\left(100 \mathrm{MHz}, \mathrm{CDCl}_{3}\right) \delta 167.2,158.9,141.1,137.8$, 131.4, 130.6, 124.22, 124.18, 52.7; HRMS (ESI-TOF) m/z: $[\mathrm{M}+\mathrm{H}]^{+}$calcd for $\mathrm{C}_{21} \mathrm{H}_{16} \mathrm{Br}_{2} \mathrm{NO}_{4}$ 503.9441, found 503.9442.

Diethyl 2,6-diphenylpyridine-3,5-dicarboxylate (5s). ${ }^{3}$ Eluent: $\mathrm{CH}_{2} \mathrm{Cl}_{2} / \mathrm{PE}$ 67:33; 10 h; yield: $23 \mathrm{mg}, 30 \%$; white solid, mp 50-52 ${ }^{\circ} \mathrm{C}$ (lit. mp 52-55 $\left.{ }^{\circ} \mathrm{C}\right) ;{ }^{1} \mathrm{H}$ NMR $(400$ $\left.\mathrm{MHz}, \mathrm{CDCl}_{3}\right) \delta 8.55(\mathrm{~s}, 1 \mathrm{H}), 7.64-7.62(\mathrm{~m}, 4 \mathrm{H}), 7.46-7.43(\mathrm{~m}, 6 \mathrm{H}), 4.21(\mathrm{q}, J=7.2$ $\mathrm{Hz}, 4 \mathrm{H}), 1.10(\mathrm{t}, J=7.2 \mathrm{~Hz}, 6 \mathrm{H}) ;{ }^{13} \mathrm{C} \mathrm{NMR}\left(100 \mathrm{MHz}, \mathrm{CDCl}_{3}\right) \delta 167.4,159.8,140.3$, 139.4, 129.2, 128.9, 128.1, 124.8, 61.7, 13.7; HRMS (ESI-TOF) m/z: $[\mathrm{M}+\mathrm{H}]^{+}$calcd for $\mathrm{C}_{23} \mathrm{H}_{22} \mathrm{NO}_{4} 376.1543$, found 376.1546 .

9. Synthesis of Compound 5a from 4a. A solution of 4 a $(75.1 \mathrm{mg}, 0.2 \mathrm{mmol})$ in DMF (2 mL) was heated at $130{ }^{\circ} \mathrm{C}$ with a heating mantle for $1.5 \mathrm{~h}$, then quenched with $\mathrm{H}_{2} \mathrm{O}(10 \mathrm{~mL})$, and extracted with EtOAc $(10 \mathrm{~mL} \times 3)$. The combined organic layer was dried with anhydrous $\mathrm{Na}_{2} \mathrm{SO}_{4}$, concentrated and purified by silica gel column chromatography, eluting with $\mathrm{CH}_{2} \mathrm{Cl}_{2} / \mathrm{PE}$ (67:33) to give 5a as a white solid (66.2 mg, 95\%).

10. Synthesis of Compound 5a from 2a. A mixture containing 2a (100.7 mg, 0.2 mmol) and $\mathrm{K}_{2} \mathrm{CO}_{3}(53 \mathrm{mg}, 0.4 \mathrm{mmol})$ in DMF $(2 \mathrm{~mL})$ was heated at $130{ }^{\circ} \mathrm{C}$ with a heating mantle for $2 \mathrm{~h}$, then quenched with $\mathrm{H}_{2} \mathrm{O}(10 \mathrm{~mL})$, and extracted with EtOAc 
$(10 \mathrm{~mL} \times 3)$. The combined organic layer was dried with anhydrous $\mathrm{Na}_{2} \mathrm{SO}_{4}$, concentrated and purified through silica gel column chromatography, eluting with $\mathrm{CH}_{2} \mathrm{Cl}_{2} / \mathrm{PE}(67: 33)$ to give $\mathbf{5 a}$ as a white solid (64.8 $\left.\mathrm{mg}, 93 \%\right)$.

\section{X-ray Structures and Data of Compounds 2a, 3d, 4f and 5a.}

The data of compounds (2a, 3d, 4f and 5a) was collected on a SuperNova diffractometer with graphite monochromated $\mathrm{Cu} \mathrm{K}_{\alpha}$ radiation $(\lambda=1.54178 \AA)$ at 293(2) K. The data were modified for Lorentz and polarization effects. The structures were determined by immediate methods and refined with a full-matrix least-squares technique based on $\mathrm{F}^{2}$ with the SHELXL-2014 software package. ${ }^{4}$ Hydrogen atoms were placed at calculated positions and refined as riding atoms with isotropic displacement parameters.

\subsection{X-ray Structure and Data of Compound 2a. Preparation of the single} crystal: Compound 2a was taken in 1:1:3 DCE/EtOAc/hexane $(5 \mathrm{~mL})$ and the solution was kept for 5 days at room temperature. The solvent was slowly evaporated and colorless crystal was formed. 


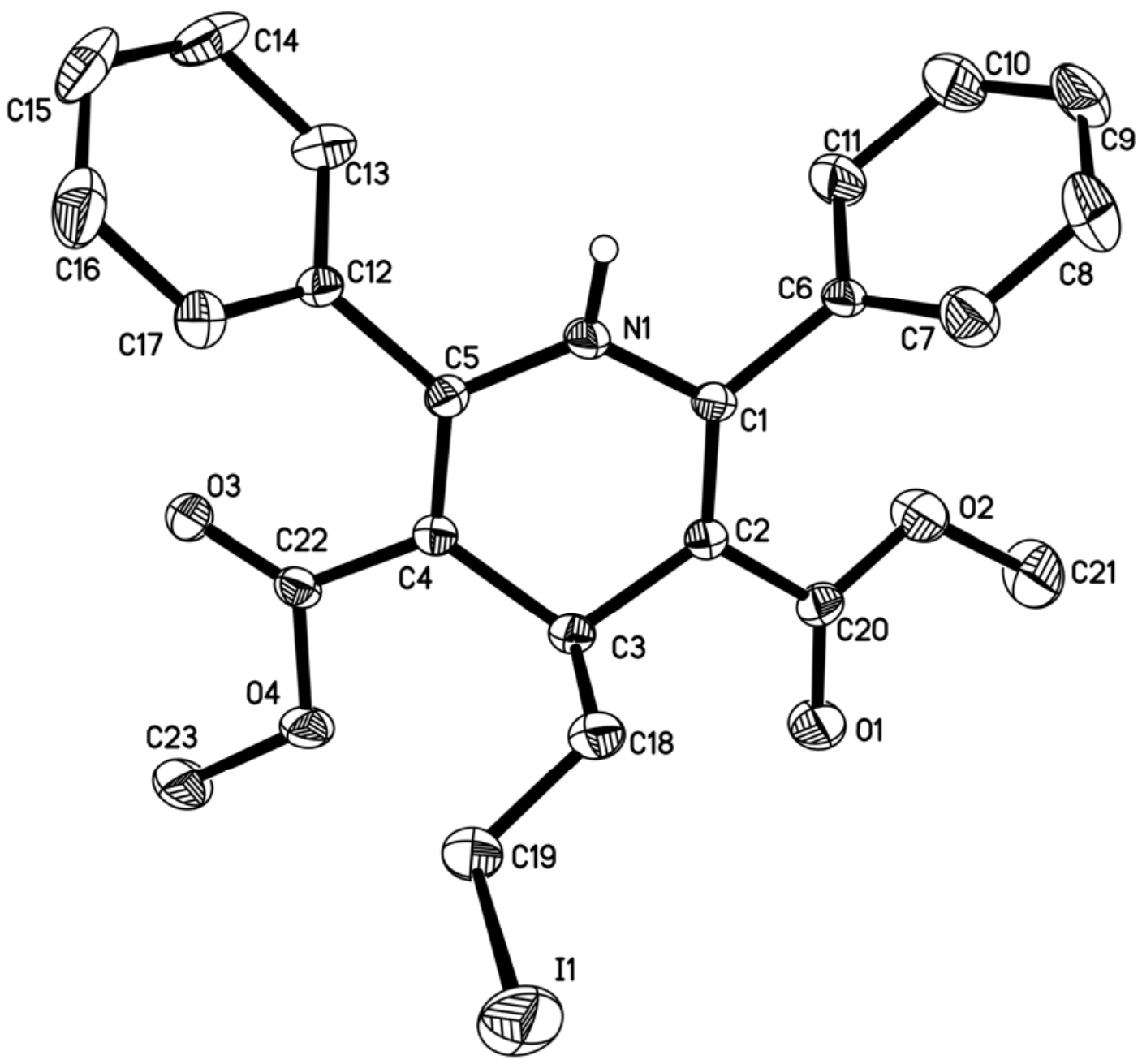

Figure S1. Molecular structure of 2a showing 30\% probability displacement ellipsoids

Table S1. Crystal Parameters and Structure Refinement for Compound 2a

Empirical formula

Formula weight

Temperature/K

Crystal system

Space group

$\mathrm{a} / \AA$
$\mathrm{C}_{23} \mathrm{H}_{22} \mathrm{INO}_{4}$

503.31

293(2)

orthorhombic

$\mathrm{P} 2{ }_{12}{ }_{21}$

$8.58735(15)$ 


\begin{tabular}{|c|c|}
\hline $\mathrm{b} / \AA$ & $10.58125(19)$ \\
\hline $\mathrm{c} / \AA$ & $24.1809(4)$ \\
\hline$\alpha /^{\circ}$ & 90 \\
\hline$\beta /{ }^{\circ}$ & 90 \\
\hline$\gamma /{ }^{\circ}$ & 90 \\
\hline Volume $/ \AA^{3}$ & $2197.20(7)$ \\
\hline $\mathrm{Z}$ & 4 \\
\hline$\rho_{\text {calc }} \mathrm{g} / \mathrm{cm}^{3}$ & 1.522 \\
\hline$\mu / \mathrm{mm}^{-1}$ & 11.682 \\
\hline $\mathrm{F}(000)$ & 1008.0 \\
\hline Crystal size $/ \mathrm{mm}^{3}$ & $0.14 \times 0.13 \times 0.1$ \\
\hline Radiation & $\mathrm{CuK} \alpha(\lambda=1.54184)$ \\
\hline $2 \Theta$ range for data collection $/^{\circ}$ & 7.312 to 141.944 \\
\hline Index ranges & $-10 \leq \mathrm{h} \leq 8,-12 \leq \mathrm{k} \leq 12,-24 \leq 1 \leq 29$ \\
\hline Reflections collected & 8486 \\
\hline Independent reflections & $3705\left[\mathrm{R}_{\text {int }}=0.0379, \mathrm{R}_{\text {sigma }}=0.0366\right]$ \\
\hline Data/restraints/parameters & $3705 / 1 / 268$ \\
\hline Goodness-of-fit on $\mathrm{F}^{2}$ & 1.027 \\
\hline Final $R$ indexes $[\mathrm{I}>=2 \sigma(\mathrm{I})]$ & $\mathrm{R}_{1}=0.0380, \mathrm{wR}_{2}=0.0993$ \\
\hline Final $\mathrm{R}$ indexes [all data] & $\mathrm{R}_{1}=0.0412, \mathrm{wR}_{2}=0.1027$ \\
\hline Largest diff. peak/hole / e $\AA^{-3}$ & $0.88 /-0.71$ \\
\hline
\end{tabular}

11.2. X-ray Structure and Data of Compound 3d. Preparation of the single crystal: Compound 3d was taken in 1:4 $\mathrm{CH}_{2} \mathrm{Cl}_{2} /$ hexane $(5 \mathrm{~mL})$ and the solution was 
kept for 2 days at room temperature. The solvent was slowly evaporated and colorless crystal was formed.

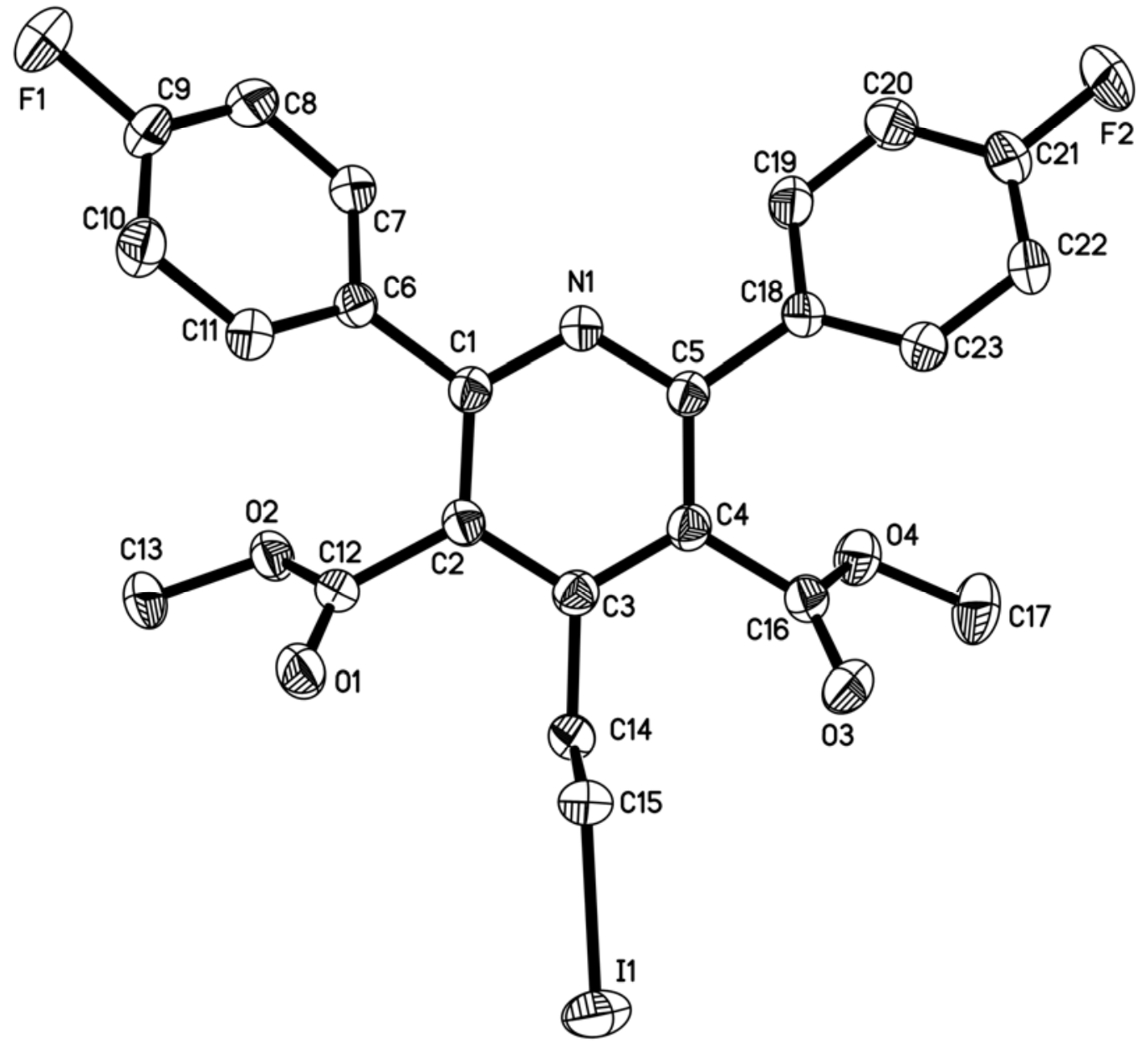

Figure S2. Molecular structure of 3d showing 30\% probability displacement ellipsoids

Table S2. Crystal Parameters and Structure Refinement for Compound 3d

\begin{tabular}{ll}
\hline Empirical formula & $\mathrm{C}_{23} \mathrm{H}_{18} \mathrm{~F}_{2} \mathrm{INO}_{4}$ \\
Formula weight & 537.28 \\
Temperature/K & $293(2)$ \\
Crystal system & monoclinic \\
Space group & $\mathrm{P} 21 / \mathrm{n}$ \\
\hline
\end{tabular}




\begin{tabular}{|c|c|}
\hline $\mathrm{a} / \AA$ & $8.8548(5)$ \\
\hline $\mathrm{b} / \AA$ & $16.8548(5)$ \\
\hline $\mathrm{c} / \AA$ & $15.0254(4)$ \\
\hline$\alpha /^{\circ}$ & 90 \\
\hline$\beta /{ }^{\circ}$ & $104.285(4)$ \\
\hline$\gamma /{ }^{\circ}$ & 90 \\
\hline Volume $/ \AA^{3}$ & $2173.13(15)$ \\
\hline Z & 4 \\
\hline$\rho_{\text {calc }} / \mathrm{cm}^{3}$ & 1.642 \\
\hline$\mu / \mathrm{mm}^{-1}$ & 11.995 \\
\hline $\mathrm{F}(000)$ & 1064.0 \\
\hline Crystal size $/ \mathrm{mm}^{3}$ & $0.203 \times 0.172 \times 0.161$ \\
\hline Radiation & $\operatorname{CuK} \alpha(\lambda=1.54184)$ \\
\hline $2 \Theta$ range for data collection $/^{\circ}$ & 8.024 to 134.156 \\
\hline Index ranges & $-10 \leq \mathrm{h} \leq 10,-12 \leq \mathrm{k} \leq 20,-17 \leq 1 \leq 17$ \\
\hline Reflections collected & 7914 \\
\hline Independent reflections & $3865\left[\mathrm{R}_{\text {int }}=0.0289, \mathrm{R}_{\text {sigma }}=0.0406\right]$ \\
\hline Data/restraints/parameters & $3865 / 0 / 283$ \\
\hline Goodness-of-fit on $\mathrm{F}^{2}$ & 1.025 \\
\hline Final $\mathrm{R}$ indexes $[\mathrm{I}>=2 \sigma(\mathrm{I})]$ & $\mathrm{R}_{1}=0.0449, \mathrm{wR}_{2}=0.1176$ \\
\hline Final $\mathrm{R}$ indexes [all data] & $\mathrm{R}_{1}=0.0575, \mathrm{wR}_{2}=0.1295$ \\
\hline Largest diff. peak/hole / e $\AA^{-3}$ & $0.99 /-0.91$ \\
\hline
\end{tabular}

11.3. X-ray Structure and Data of Compound 4f. Preparation of the single crystal: 
Compound $4 \mathbf{f}$ was taken in 1:4 $\mathrm{CH}_{2} \mathrm{Cl}_{2} /$ hexane $(5 \mathrm{~mL})$ and the solution was kept for 2 days at room temperature. The solvent was slowly evaporated and colorless crystal was formed.

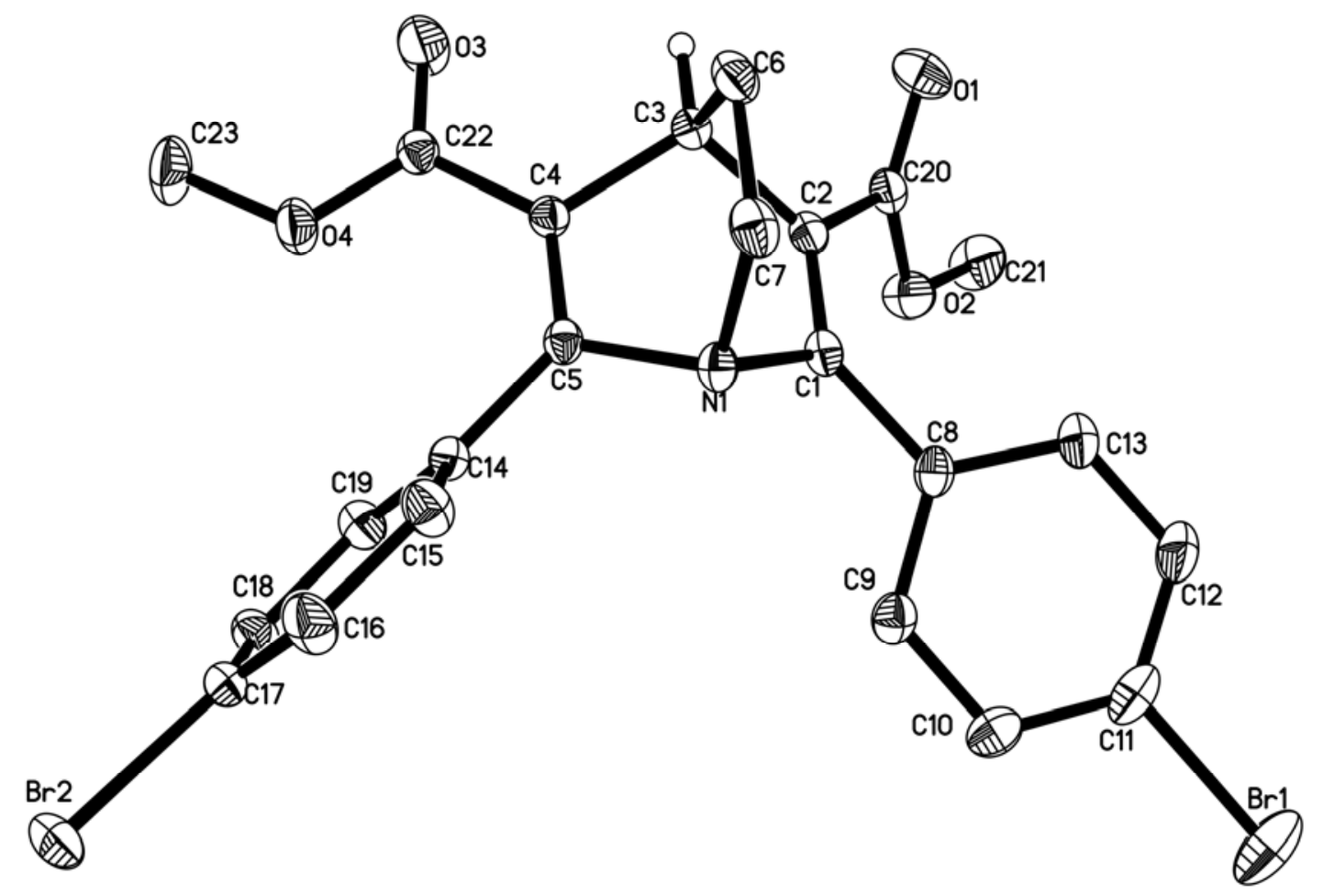

Figure S3. Molecular structure of $\mathbf{4 f}$ showing $30 \%$ probability displacement

ellipsoids

Table S3. Crystal Parameters and Structure Refinement for Compound $\mathbf{4 f}$

Empirical formula

Formula weight

Temperature/K

Crystal system

Space group

$\mathrm{a} / \AA$

$\mathrm{b} / \AA$
$\mathrm{C}_{23} \mathrm{H}_{19} \mathrm{Br}_{2} \mathrm{NO}_{4}$

533.21

293(2)

monoclinic

$\mathrm{P} 21 / \mathrm{c}$

$22.1475(5)$

$8.6327(2)$ 


\begin{tabular}{|c|c|}
\hline $\mathrm{c} / \AA ̊$ & $11.7243(4)$ \\
\hline$\alpha /^{\circ}$ & 90 \\
\hline$\beta /^{\circ}$ & $101.930(3)$ \\
\hline$\gamma /{ }^{\circ}$ & 90 \\
\hline Volume $/ \AA^{3}$ & $2193.20(11)$ \\
\hline $\mathrm{Z}$ & 4 \\
\hline$\rho_{\text {calcg }} / \mathrm{cm}^{3}$ & 1.615 \\
\hline$\mu / \mathrm{mm}^{-1}$ & 4.937 \\
\hline $\mathrm{F}(000)$ & 1064.0 \\
\hline Crystal size $/ \mathrm{mm}^{3}$ & $0.15 \times 0.1 \times 0.08$ \\
\hline Radiation & $\operatorname{CuK} \alpha(\lambda=1.54184)$ \\
\hline $2 \Theta$ range for data collection $/{ }^{\circ}$ & 8.16 to 134.108 \\
\hline Index ranges & $-26 \leq \mathrm{h} \leq 26,-5 \leq \mathrm{k} \leq 10,-14 \leq 1 \leq 13$ \\
\hline Reflections collected & 8085 \\
\hline Independent reflections & $3909\left[\mathrm{R}_{\text {int }}=0.0298, \mathrm{R}_{\text {sigma }}=0.0415\right]$ \\
\hline Data/restraints/parameters & $3909 / 0 / 273$ \\
\hline Goodness-of-fit on $\mathrm{F}^{2}$ & 1.039 \\
\hline Final $R$ indexes $[\mathrm{I}>=2 \sigma(\mathrm{I})]$ & $\mathrm{R}_{1}=0.0413, \mathrm{wR}_{2}=0.0998$ \\
\hline Final $\mathrm{R}$ indexes [all data] & $\mathrm{R}_{1}=0.0542, \mathrm{wR}_{2}=0.1101$ \\
\hline Largest diff. peak/hole / e $\AA^{-3}$ & $0.41 /-0.57$ \\
\hline
\end{tabular}

11.4. X-ray Structure and Data of Compound 5a. Preparation of the single crystal: Compound 5a was taken in $1: 4 \mathrm{CH}_{2} \mathrm{Cl}_{2} /$ hexane $(5 \mathrm{~mL})$ and the solution was kept for 2 days at room temperature. The solvent was slowly evaporated and colorless 
crystal was formed.

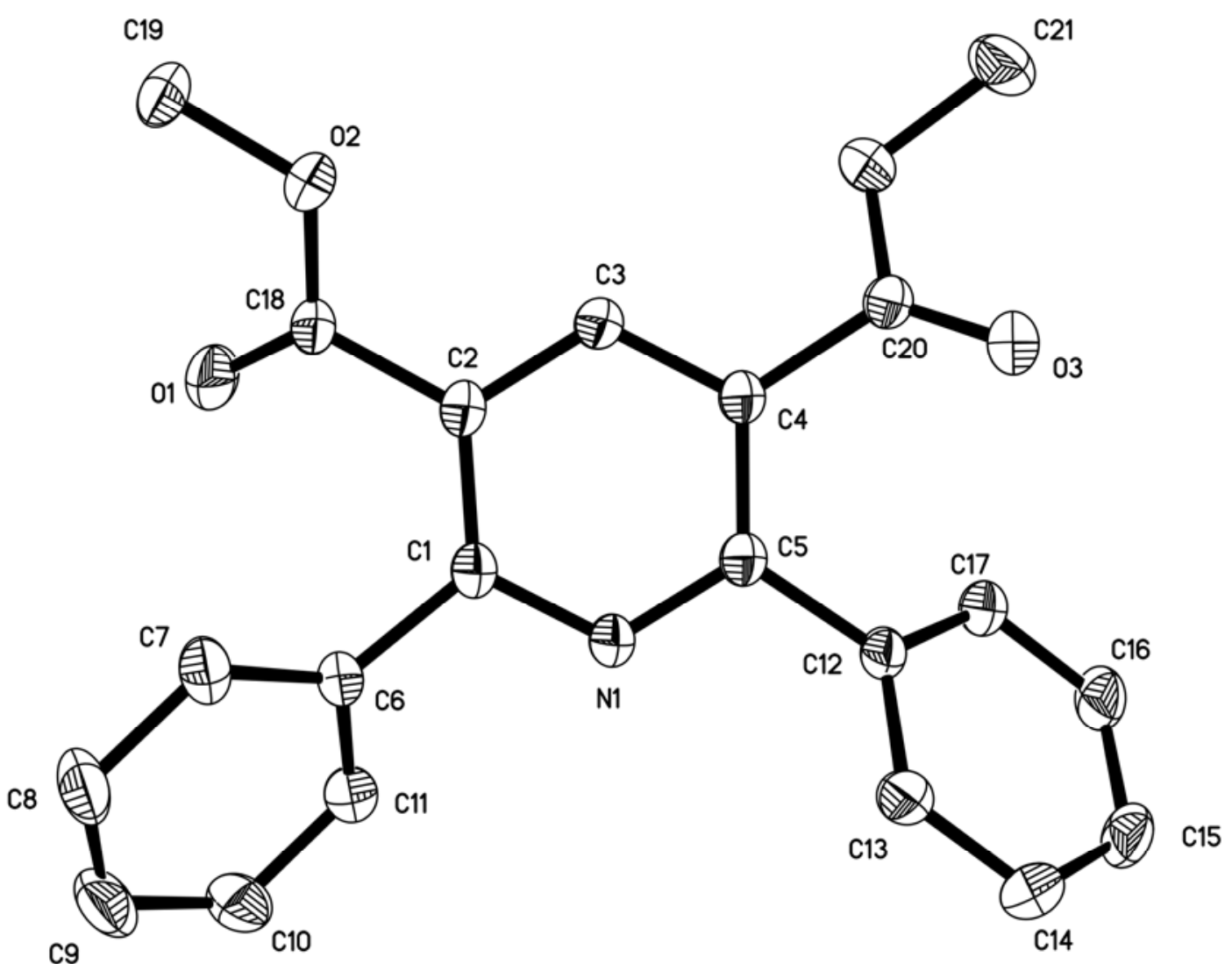

Figure S4. Molecular structure of 5a showing 30\% probability displacement

ellipsoids

Table S4. Crystal Parameters and Structure Refinement for Compound 5a

\begin{tabular}{ll}
\hline Empirical formula & $\mathrm{C}_{21} \mathrm{H}_{17} \mathrm{NO}_{4}$ \\
Formula weight & 347.36 \\
Temperature/K & $293(2)$ \\
Crystal system & monoclinic \\
Space group & $\mathrm{P} 21 / \mathrm{c}$ \\
a/ $\AA$ & $21.6254(5)$ \\
$\mathrm{b} / \AA$ & $4.15378(17)$ \\
\hline
\end{tabular}




\begin{tabular}{|c|c|}
\hline $\mathrm{c} / \AA \AA$ & $18.9245(5)$ \\
\hline$\alpha /^{\circ}$ & 90 \\
\hline$\beta /{ }^{\circ}$ & $94.369(2)$ \\
\hline$\gamma /{ }^{\circ}$ & 90 \\
\hline Volume $/ \AA^{3}$ & $1695.00(9)$ \\
\hline Z & 4 \\
\hline$\rho_{\text {calc }} / \mathrm{cm}^{3}$ & 1.361 \\
\hline$\mu / \mathrm{mm}^{-1}$ & 0.776 \\
\hline $\mathrm{F}(000)$ & 728.0 \\
\hline Crystal size $/ \mathrm{mm}^{3}$ & $0.17 \times 0.12 \times 0.1$ \\
\hline Radiation & $\mathrm{CuK} \alpha(\lambda=1.54184)$ \\
\hline $2 \Theta$ range for data collection $/^{\circ}$ & 8.2 to 142.264 \\
\hline Index ranges & $-25 \leq \mathrm{h} \leq 25,-4 \leq \mathrm{k} \leq 4,-23 \leq 1 \leq 21$ \\
\hline Reflections collected & 13329 \\
\hline Independent reflections & $3199\left[\mathrm{R}_{\text {int }}=0.0338, \mathrm{R}_{\text {sigma }}=0.0345\right]$ \\
\hline Data/restraints/parameters & $3199 / 0 / 237$ \\
\hline Goodness-of-fit on $\mathrm{F}^{2}$ & 1.082 \\
\hline Final $\mathrm{R}$ indexes $[\mathrm{I}>=2 \sigma(\mathrm{I})]$ & $\mathrm{R}_{1}=0.0441, \mathrm{wR}_{2}=0.1163$ \\
\hline Final $\mathrm{R}$ indexes [all data] & $\mathrm{R}_{1}=0.0579, \mathrm{wR}_{2}=0.1339$ \\
\hline Largest diff. peak/hole / e $\AA^{-3}$ & $0.16 /-0.20$ \\
\hline
\end{tabular}

\section{References}

1. Zhang, X.-Y.; Yang, Z.-W.; Chen, Z.; Wang, J.; Yang, D.-L.; Shen, Z.; Hu, L.-L.; 
Xie, J.-W.; Zhang, J.; Cui, H.-L. Tandem Copper-Catalyzed Propargylation/Alkyne Azacyclization/Isomerization Reaction under Microwave Irradiation: Synthesis of Fully Substituted Pyrroles. J. Org. Chem. 2016, 81, 1778-1785.

2. Tsuritani, T.; Strotman, N. A.; Yamamoto, Y.; Kawasaki, M.; Yasuda, N.; Mase, T. $\mathrm{N}$-Cyclopropylation of Indoles and Cyclic Amides with Copper(II) Reagent. Org. Lett. 2008, $10,1653-1655$.

3. Wu, X.; Zhang, J.; Liu, S.; Gao, Q.; Wu, A. An Efficient Synthesis of Polysubstituted Pyridines via C-H Oxidation and C-S Cleavage of Dimethyl Sulfoxide. Adv. Synth. Catal. 2016, 358, 218-225.

4. (a) G. M. Sheldrick, SADABS, Program for Empirical Absorption Correction of Area Detector Data, University of Göttingen, Germany, 2003; (b) G. M. Sheldrick, SHELXS-2014, Program for the Crystal Structure Solution, University of Göttingen, Germany, 2014. 


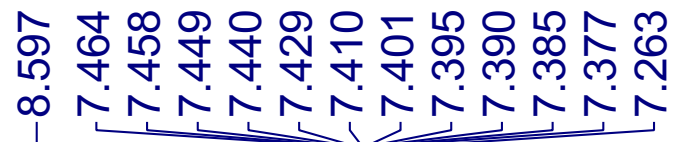

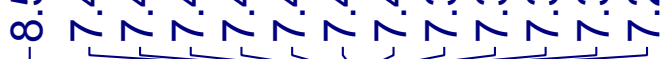

${ }^{1} \mathrm{H} \mathrm{NMR}\left(400 \mathrm{MHz}, \mathrm{CDCl}_{3}\right)$

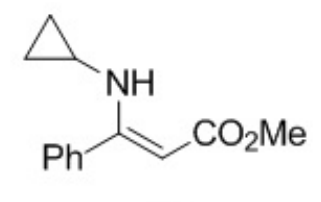

$1 \mathrm{a}$

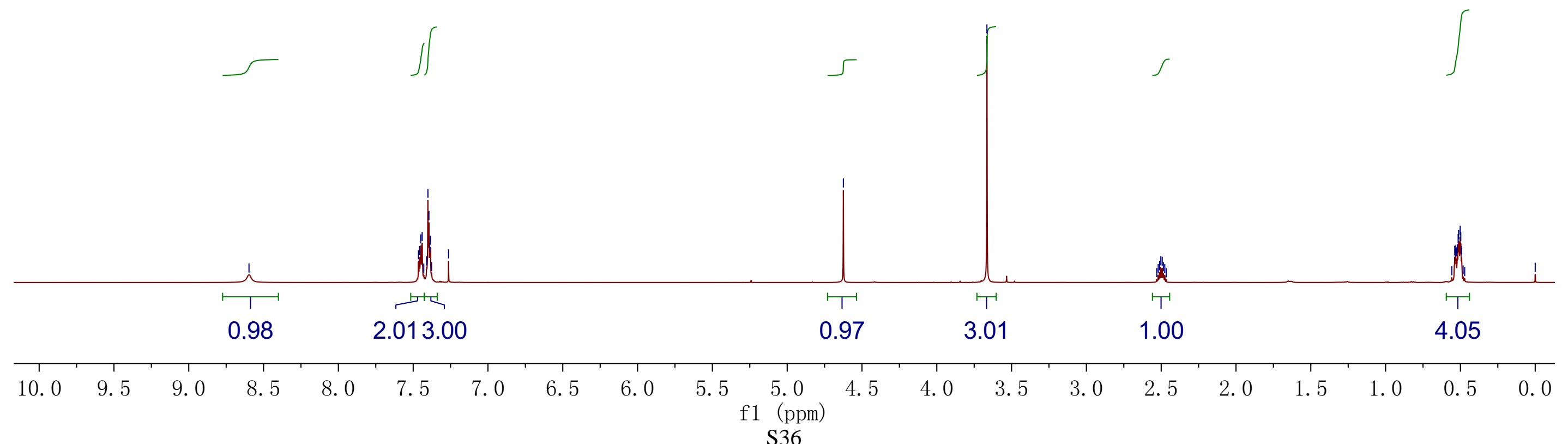


${ }^{13} \mathrm{C}$ NMR (100 MHz, $\left.\mathrm{CDCl}_{3}\right)$

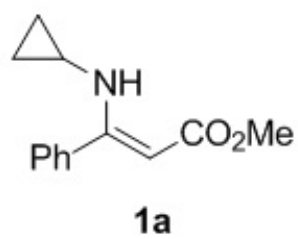




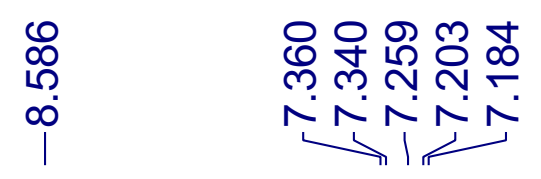

\section{${ }^{1} \mathrm{H}$ NMR (400 MHz, $\mathrm{CDCl}_{3}$ )}

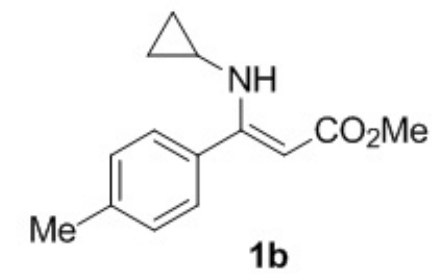

1b

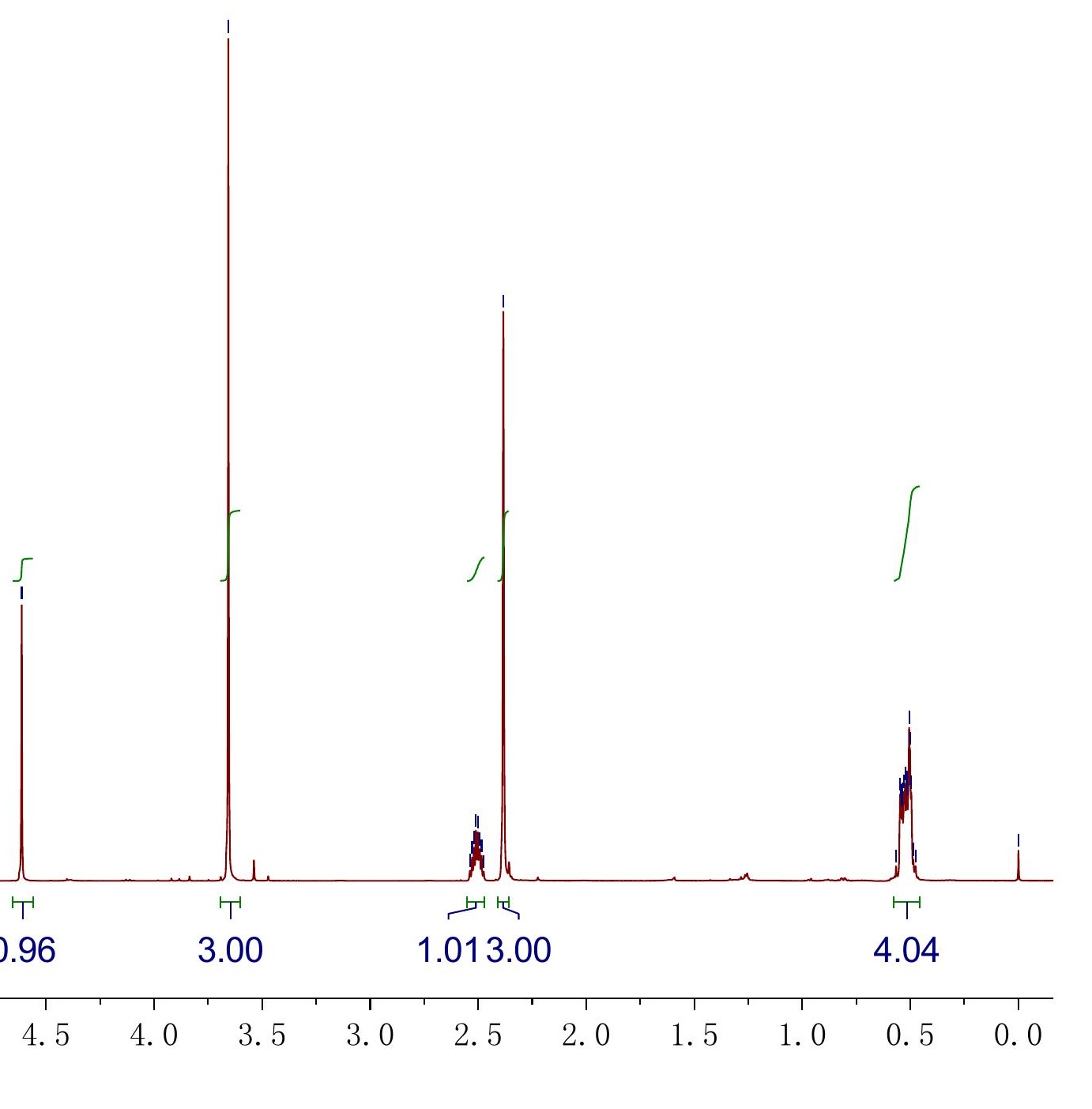


${ }^{13} \mathrm{C} \mathrm{NMR}\left(100 \mathrm{MHz}, \mathrm{CDCl}_{3}\right)$
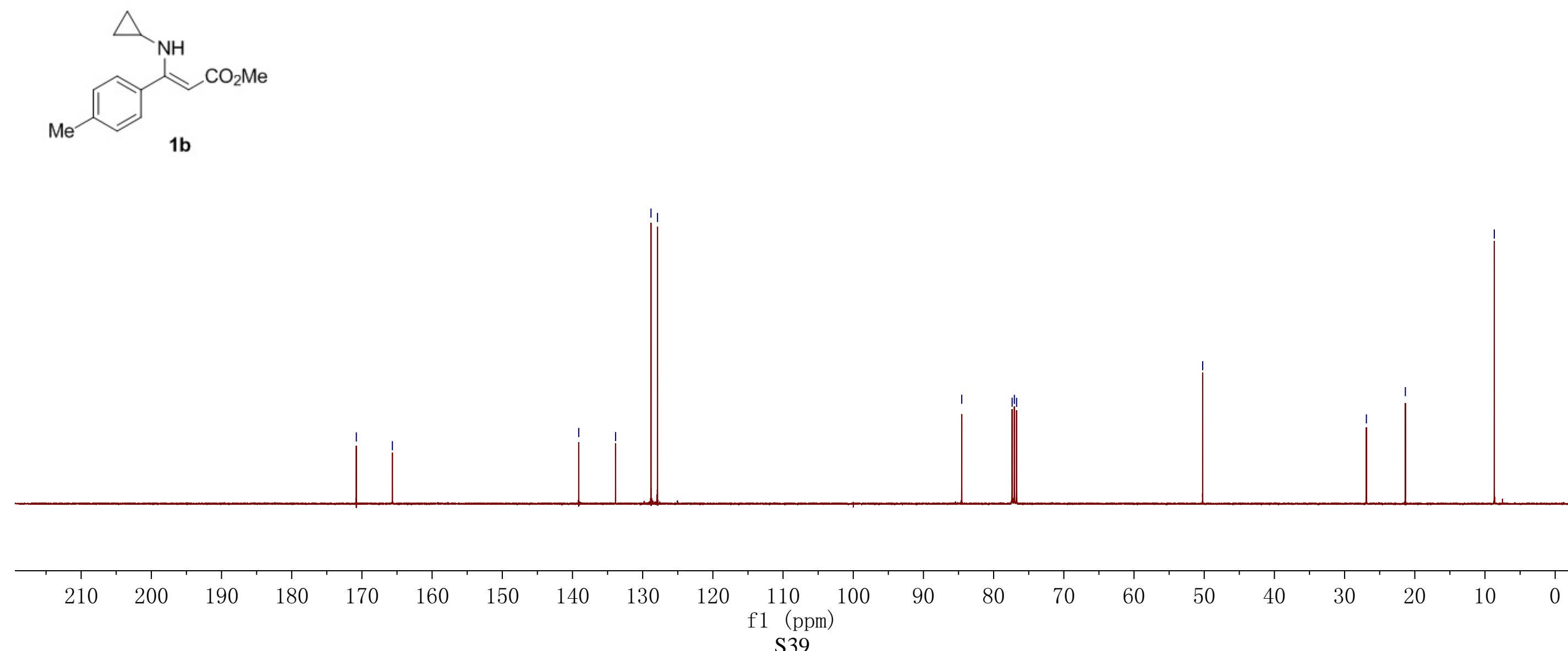
${ }^{1} \mathrm{H} \mathrm{NMR}\left(400 \mathrm{MHz}, \mathrm{CDCl}_{3}\right)$
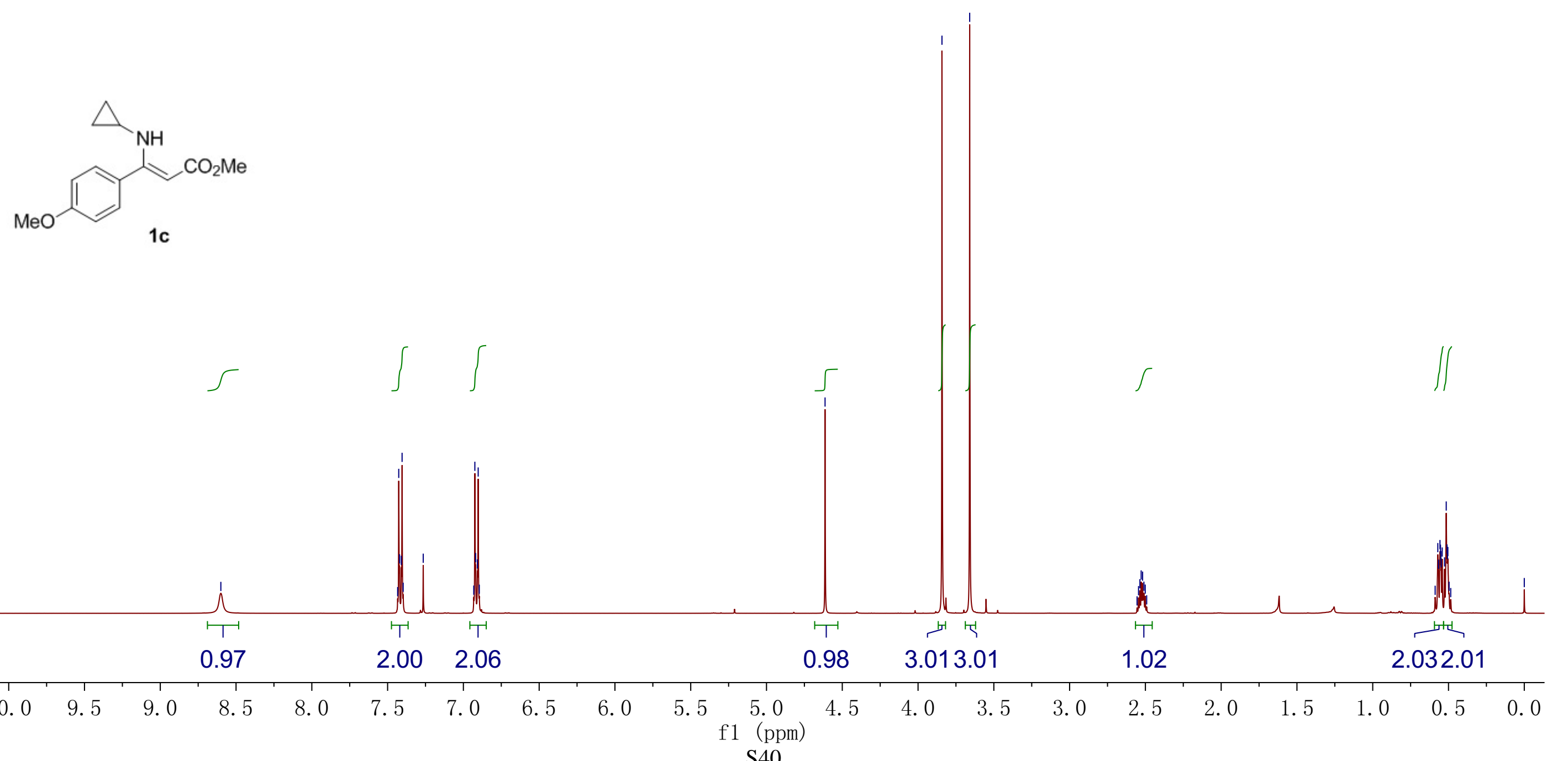
${ }^{13} \mathrm{C}$ NMR $\left(100 \mathrm{MHz}, \mathrm{CDCl}_{3}\right)$
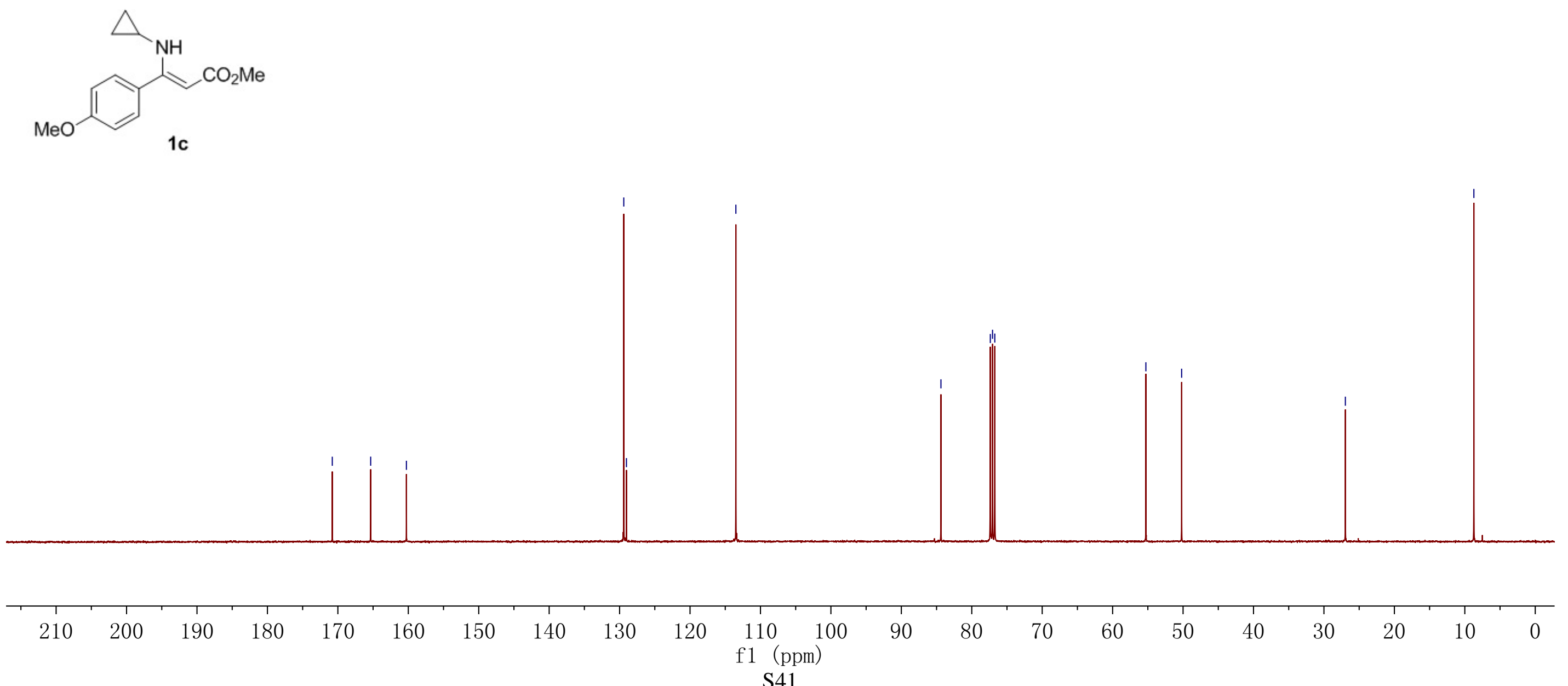
${ }^{1} \mathrm{H}$ NMR (400 MHz, $\mathrm{CDCl}_{3}$ )
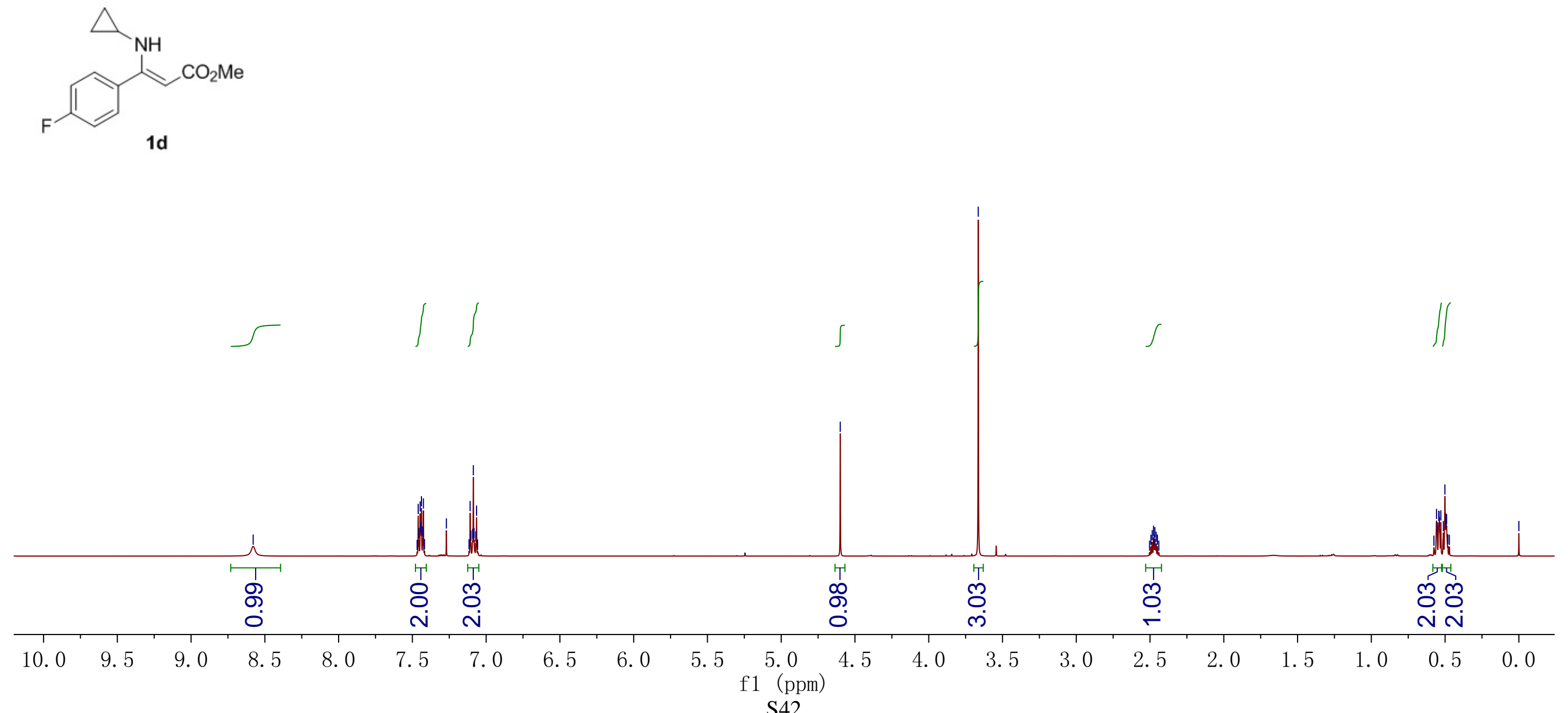
${ }^{13} \mathrm{C} \mathrm{NMR}\left(100 \mathrm{MHz}, \mathrm{CDCl}_{3}\right)$

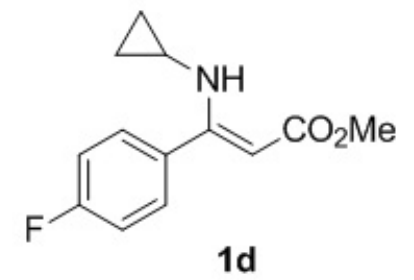

1d

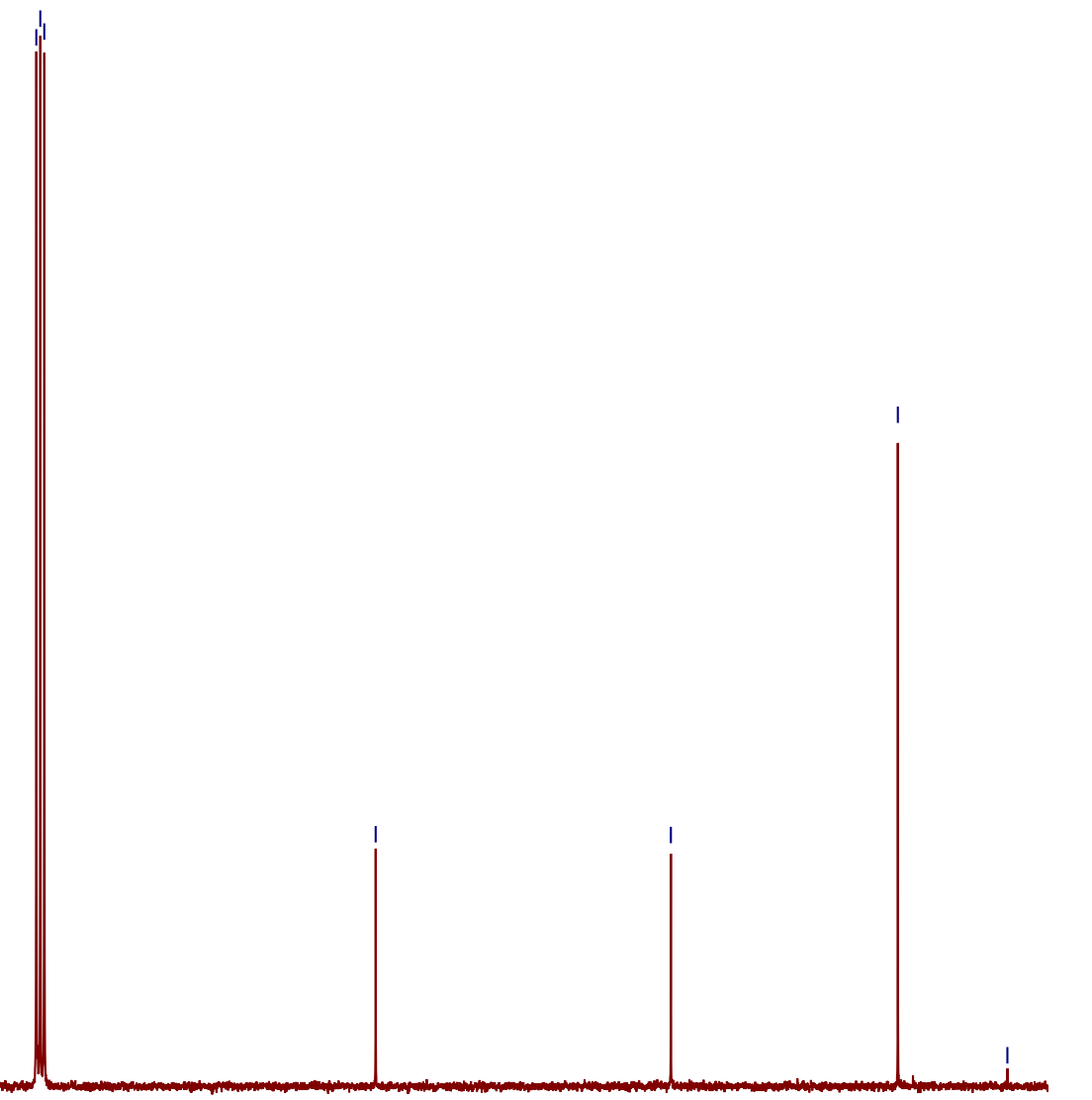


${ }^{19} \mathrm{~F}$ NMR $\left(376 \mathrm{MHz}, \mathrm{CDCl}_{3}\right)$

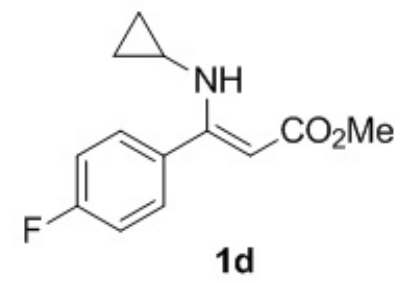

10

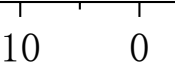

$0-10$

$-20$

$-30$

$-40$

$-50$

$-60$

$-70$

$-80$

$-90-100$

$-120$

$-140$

$-160$

$-180$ 
${ }^{1} \mathrm{H} \mathrm{NMR}\left(400 \mathrm{MHz}, \mathrm{CDCl}_{3}\right)$

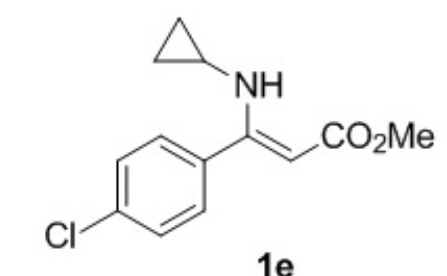

$1 e$

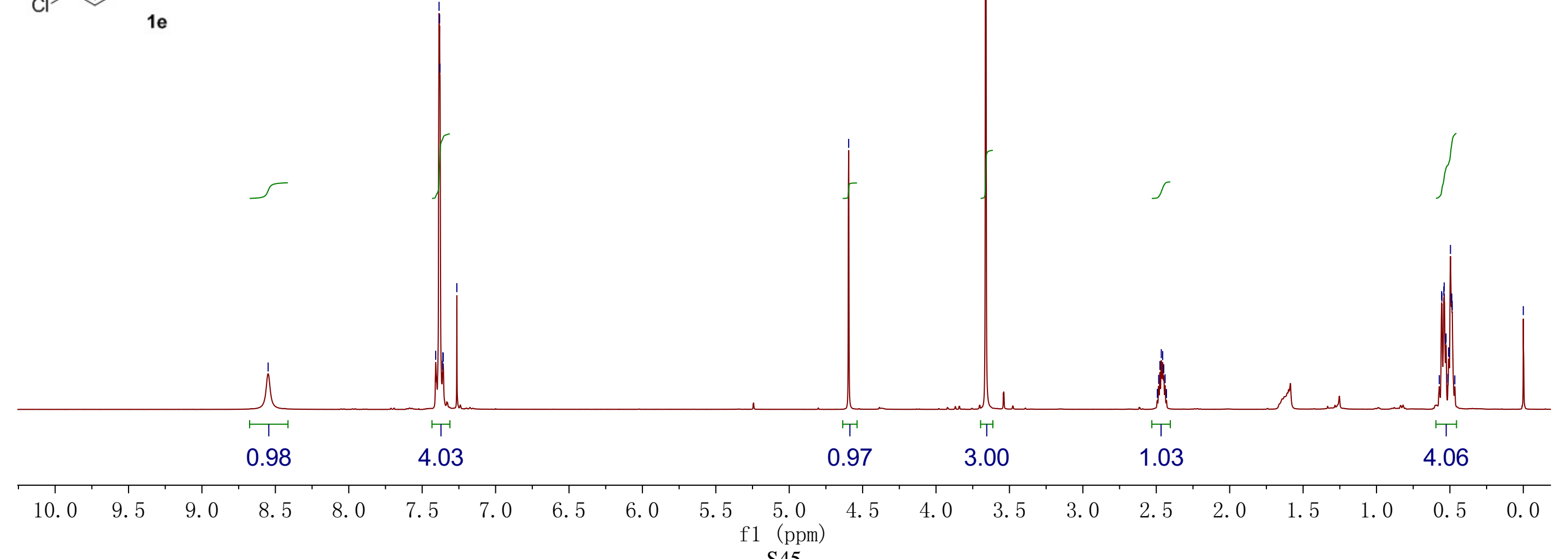


${ }^{13} \mathrm{C} \mathrm{NMR}\left(100 \mathrm{MHz}, \mathrm{CDCl}_{3}\right)$

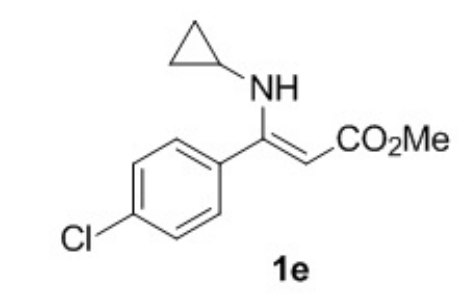

กัด

泾余

m

$\stackrel{\infty}{\stackrel{N}{\circ}}$

$\begin{array}{ll}\infty & 0 \\ \infty & 0 \\ 1 & 1\end{array}$

$1 \mathrm{e}$

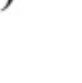


${ }^{1} \mathrm{H}$ NMR (400 MHz, $\left.\mathrm{CDCl}_{3}\right)$
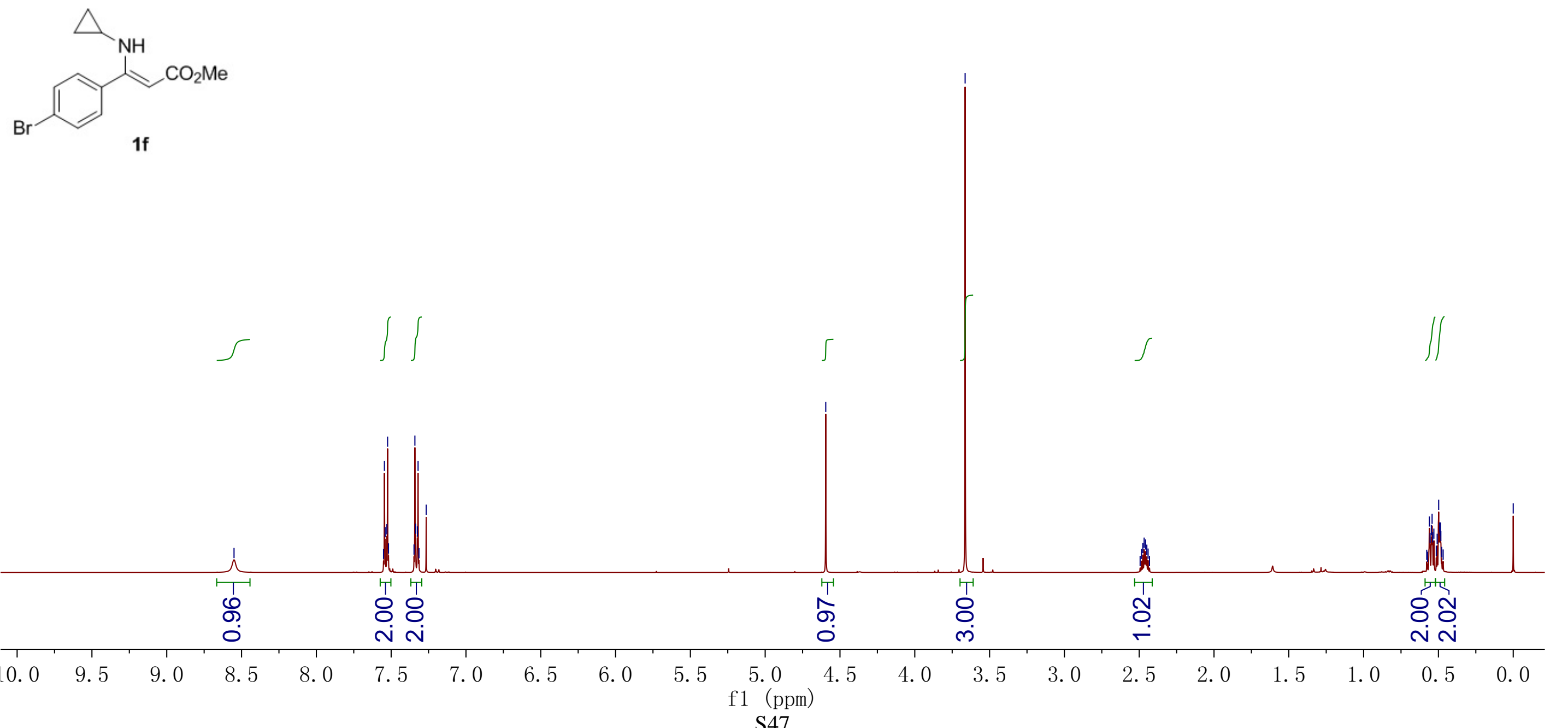
${ }^{13} \mathrm{C} \mathrm{NMR}\left(100 \mathrm{MHz}, \mathrm{CDCl}_{3}\right)$
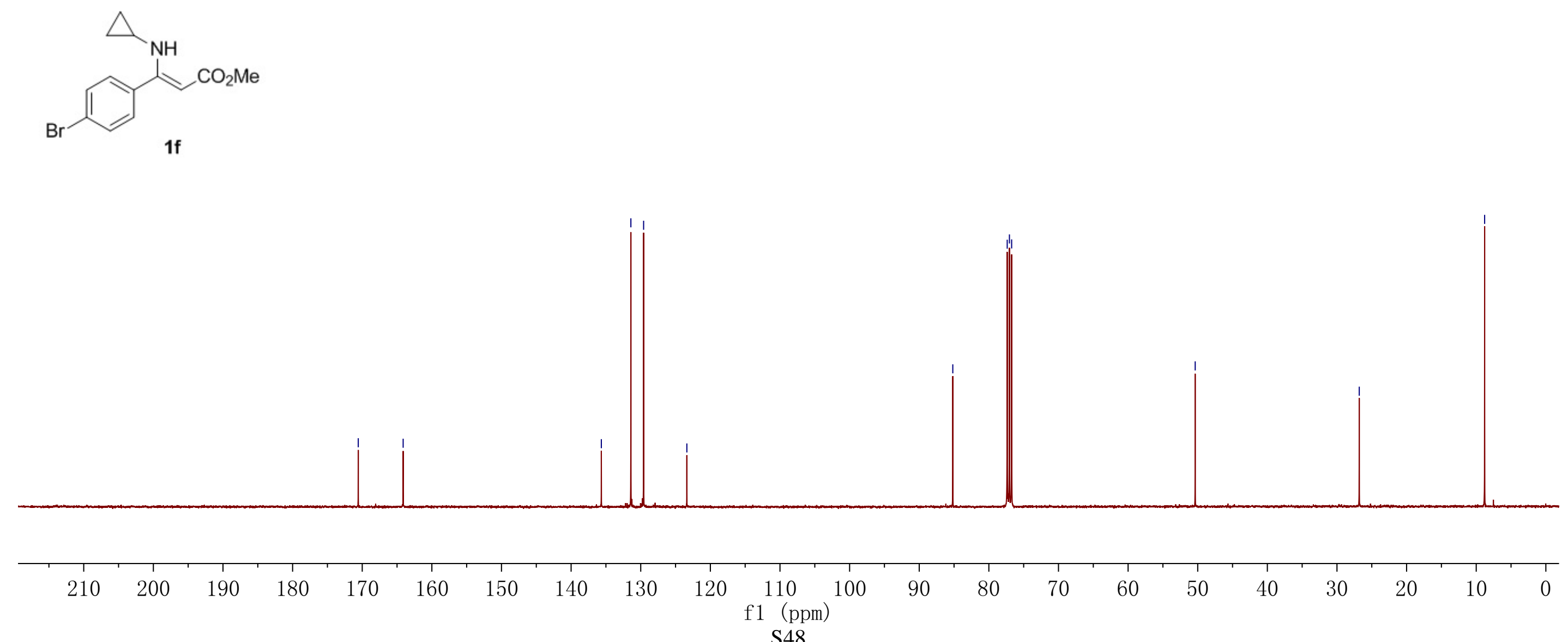


\section{${ }^{1} \mathrm{H} \mathrm{NMR}\left(400 \mathrm{MHz}, \mathrm{CDCl}_{3}\right)$}
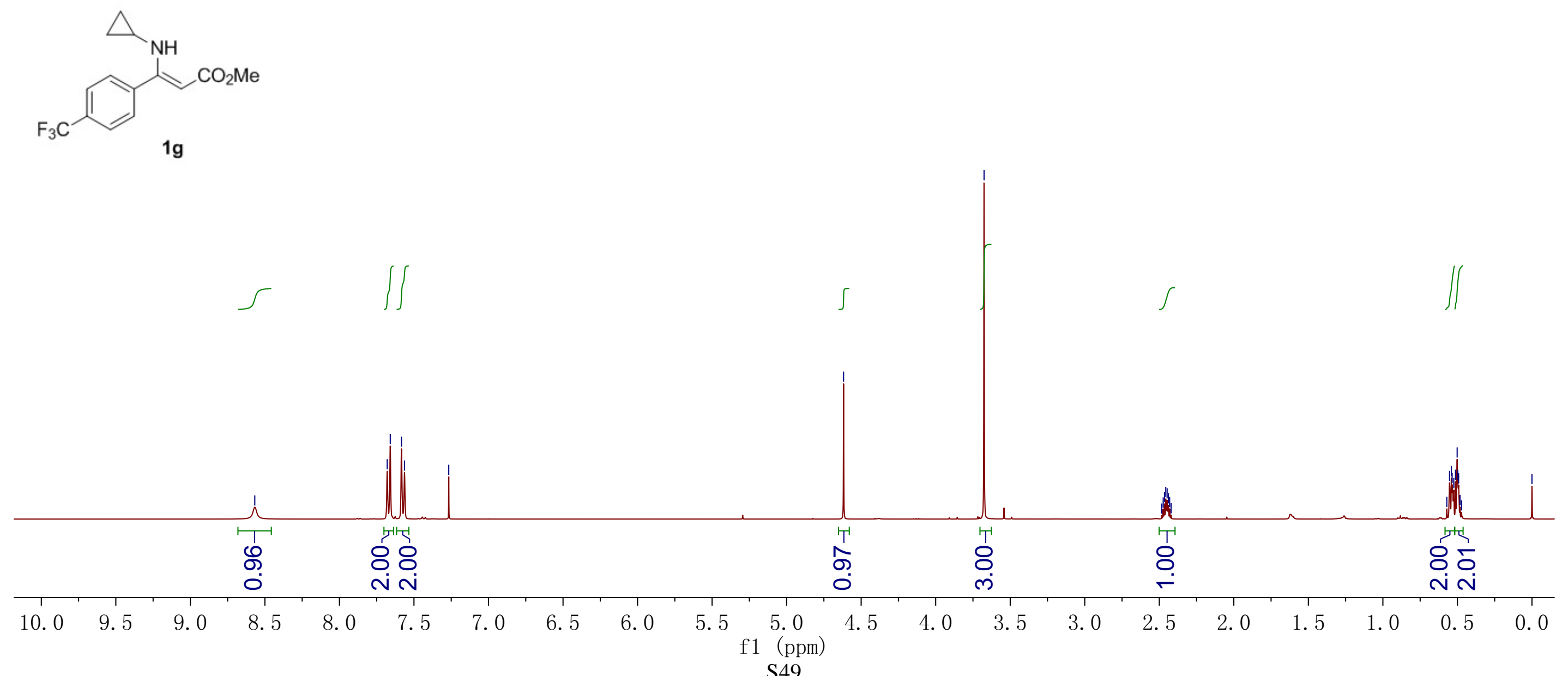
${ }^{13} \mathrm{C}$ NMR $\left(100 \mathrm{MHz}, \mathrm{CDCl}_{3}\right)$

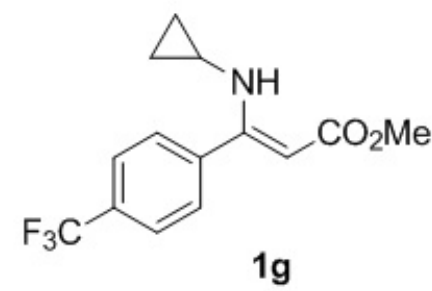

オ mo

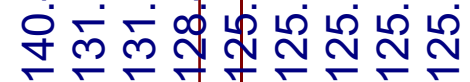
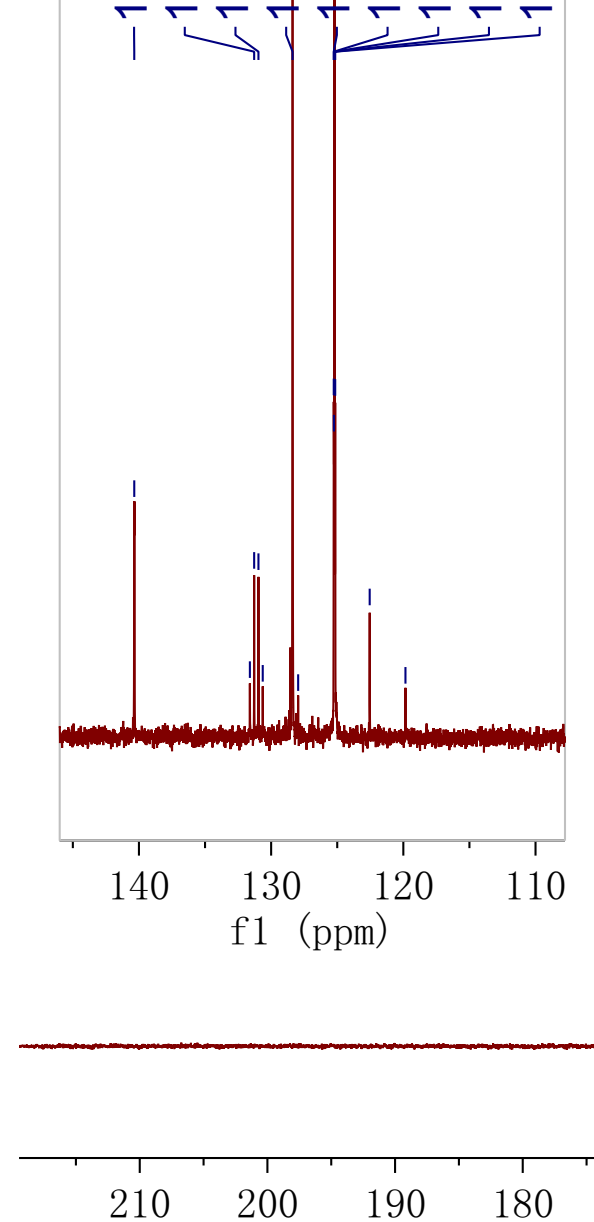

210

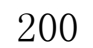

190

180

170

160

150

140

130

120 f1 (ppm) 
${ }^{19} \mathrm{~F}$ NMR (376 $\mathrm{MHz}, \mathrm{CDCl}_{3}$ )
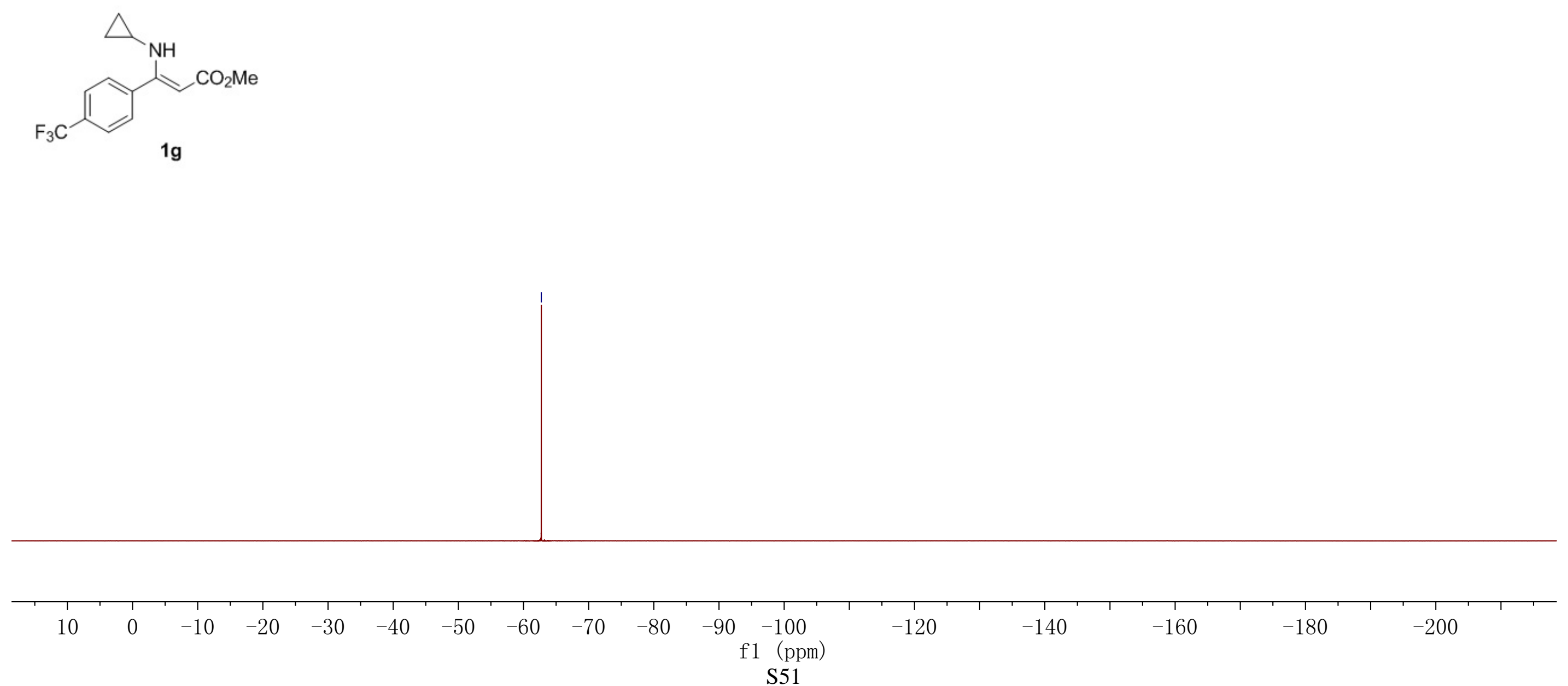


\section{${ }^{1} \mathrm{H} \mathrm{NMR}\left(400 \mathrm{MHz}, \mathrm{CDCl}_{3}\right)$}

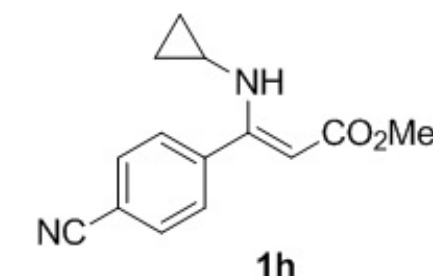

$1 \mathrm{~h}$

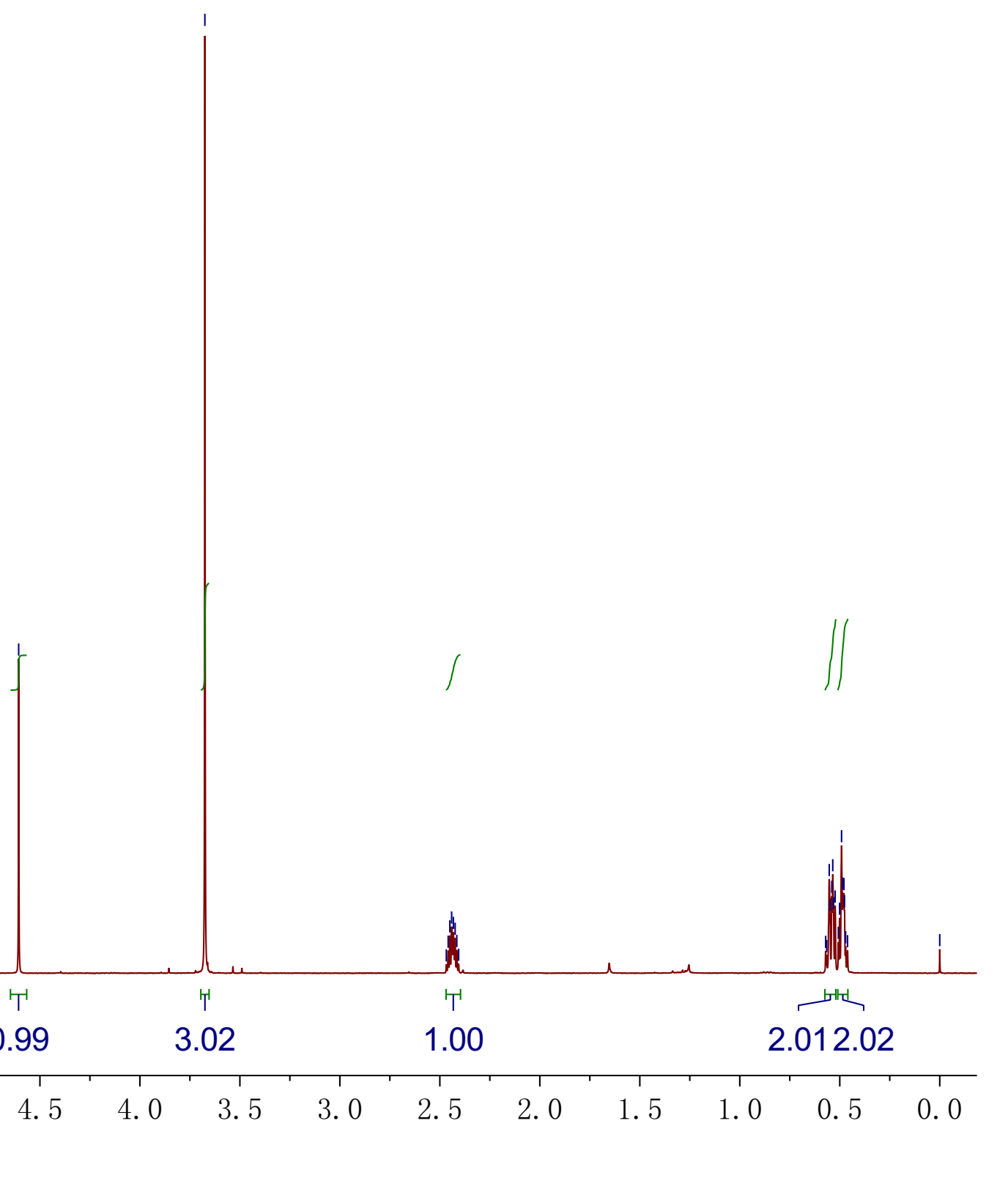




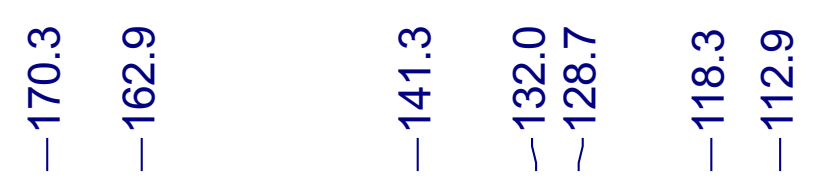

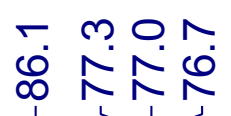

مْ

ڤัต

$\infty$

${ }^{13} \mathrm{C}$ NMR $\left(100 \mathrm{MHz}, \mathrm{CDCl}_{3}\right)$

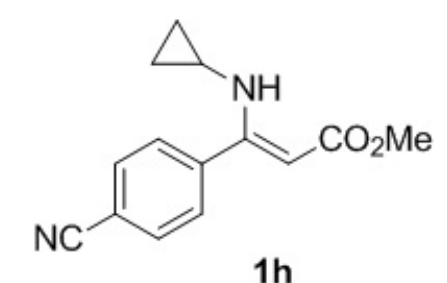

$1 \mathrm{~h}$

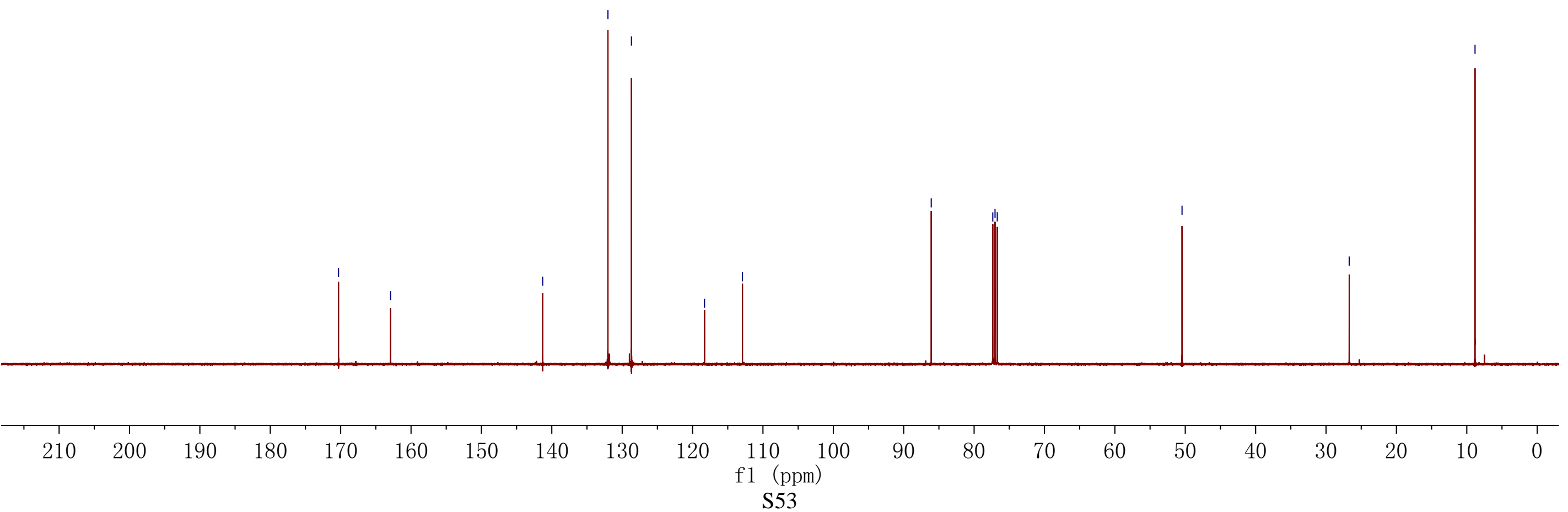


${ }^{1} \mathrm{H}$ NMR (400 MHz, $\mathrm{CDCl}_{3}$ )
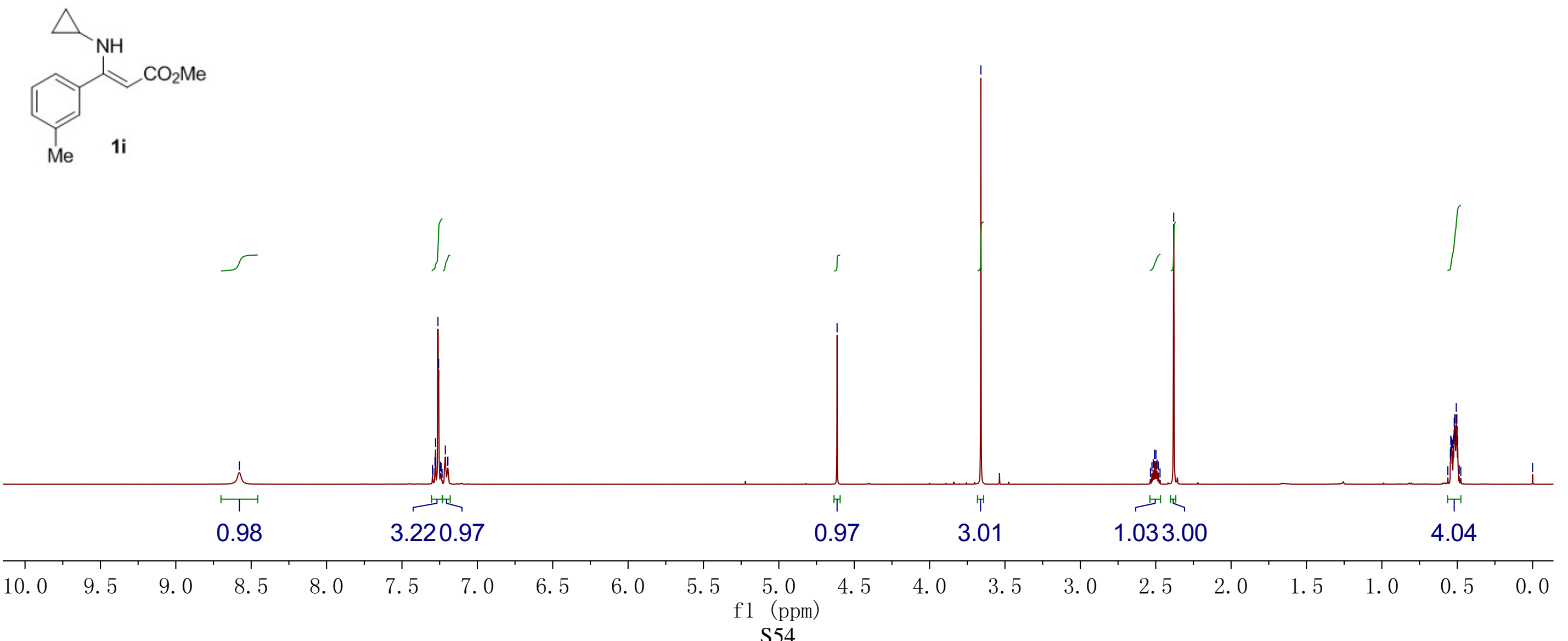
${ }^{13} \mathrm{C} \mathrm{NMR}\left(100 \mathrm{MHz}, \mathrm{CDCl}_{3}\right)$
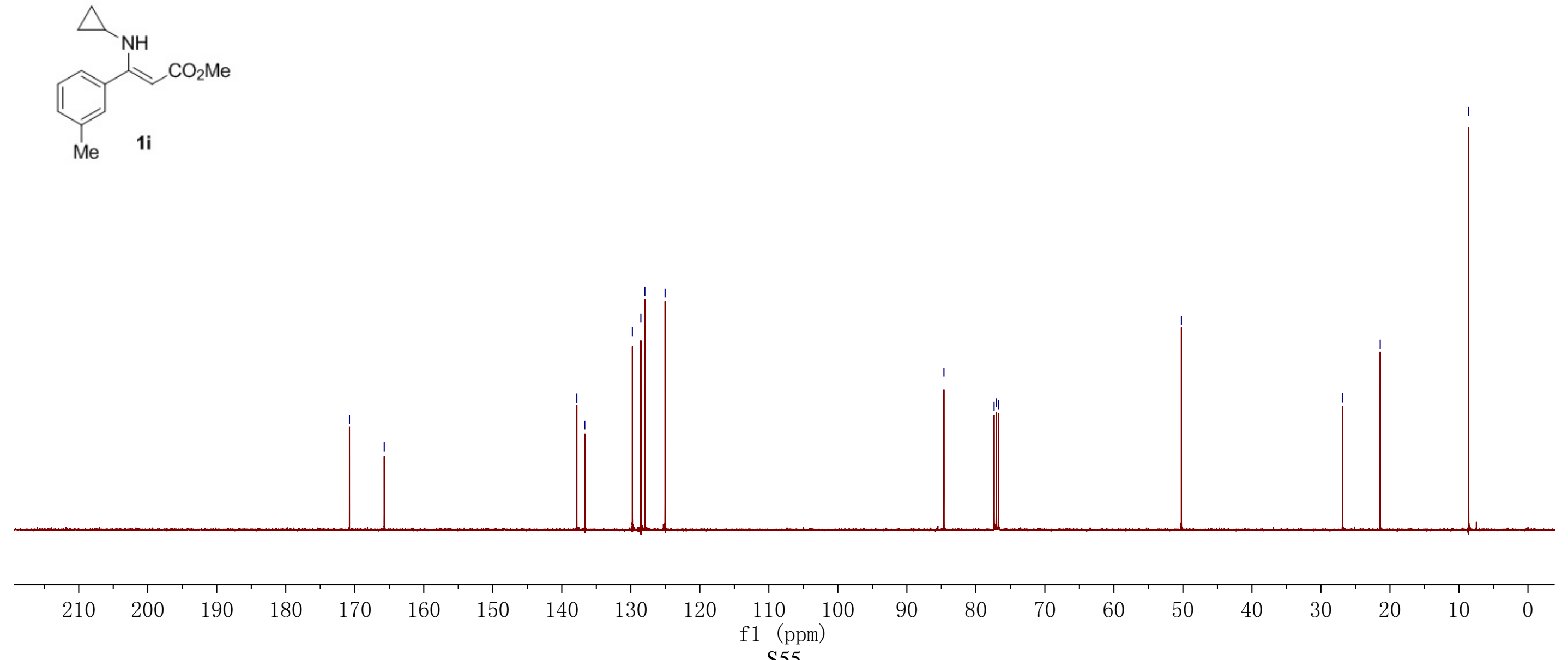
${ }^{1} \mathrm{H}$ NMR (400 MHz, $\mathrm{CDCl}_{3}$ )
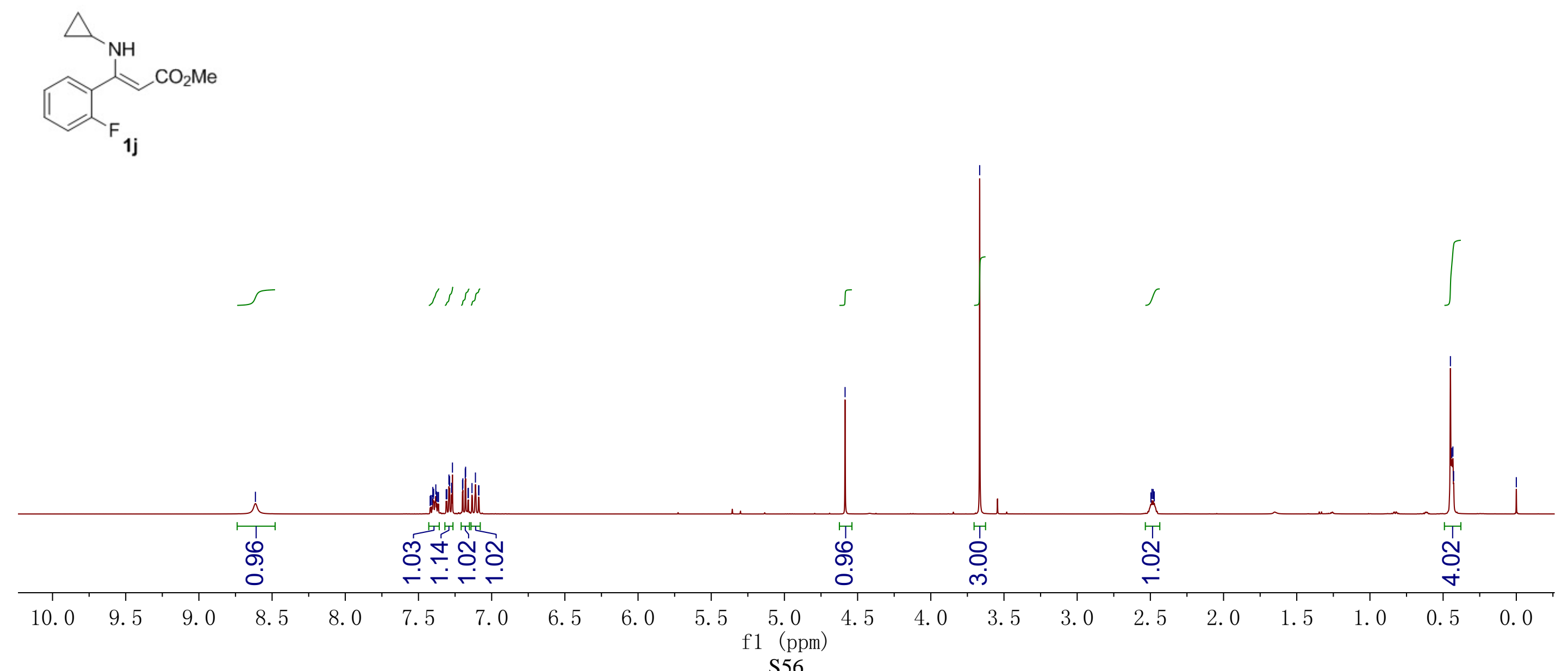
${ }^{13} \mathrm{C}$ NMR (100 MHz, $\left.\mathrm{CDCl}_{3}\right)$

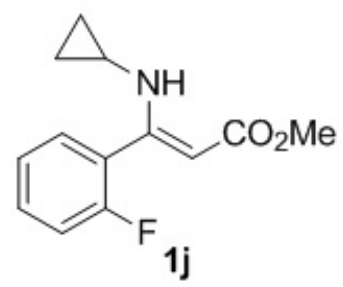


${ }^{19} \mathrm{~F}$ NMR (376 $\mathrm{MHz}, \mathrm{CDCl}_{3}$ )
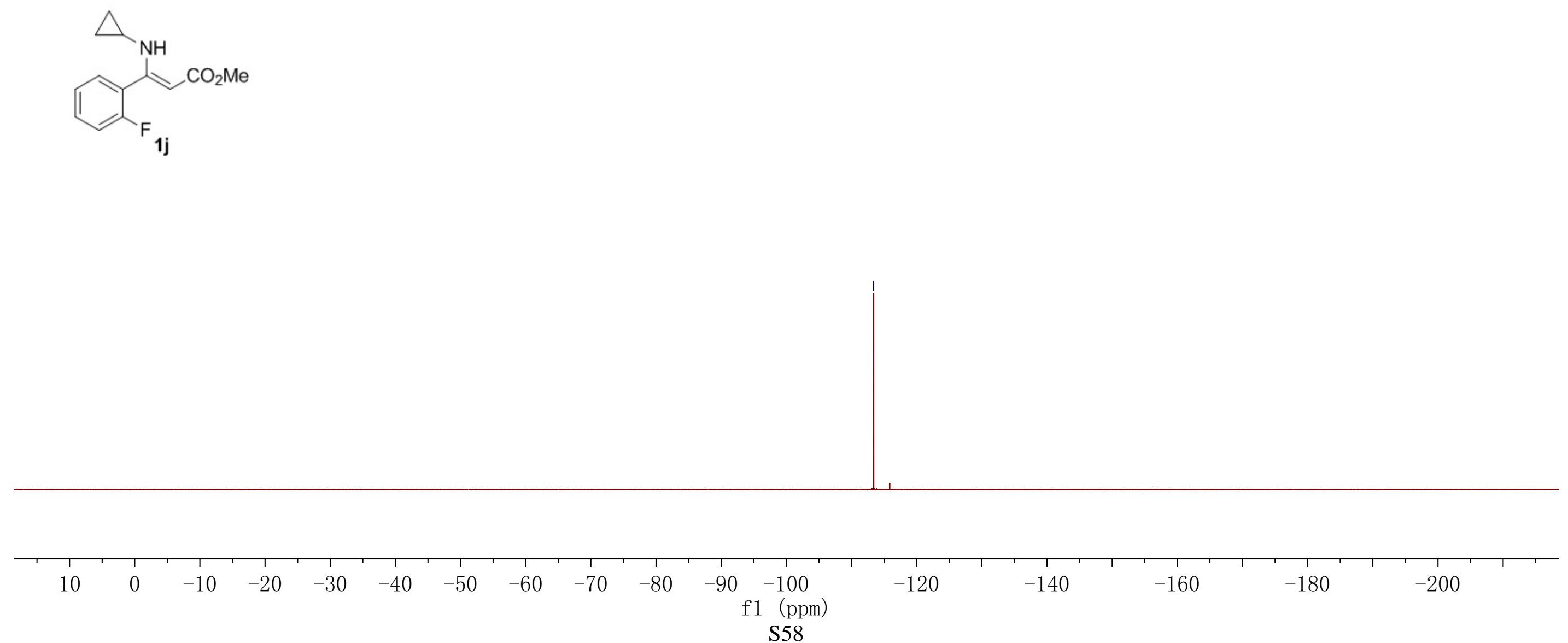
${ }^{1} \mathrm{H} \mathrm{NMR}\left(400 \mathrm{MHz}, \mathrm{CDCl}_{3}\right)$
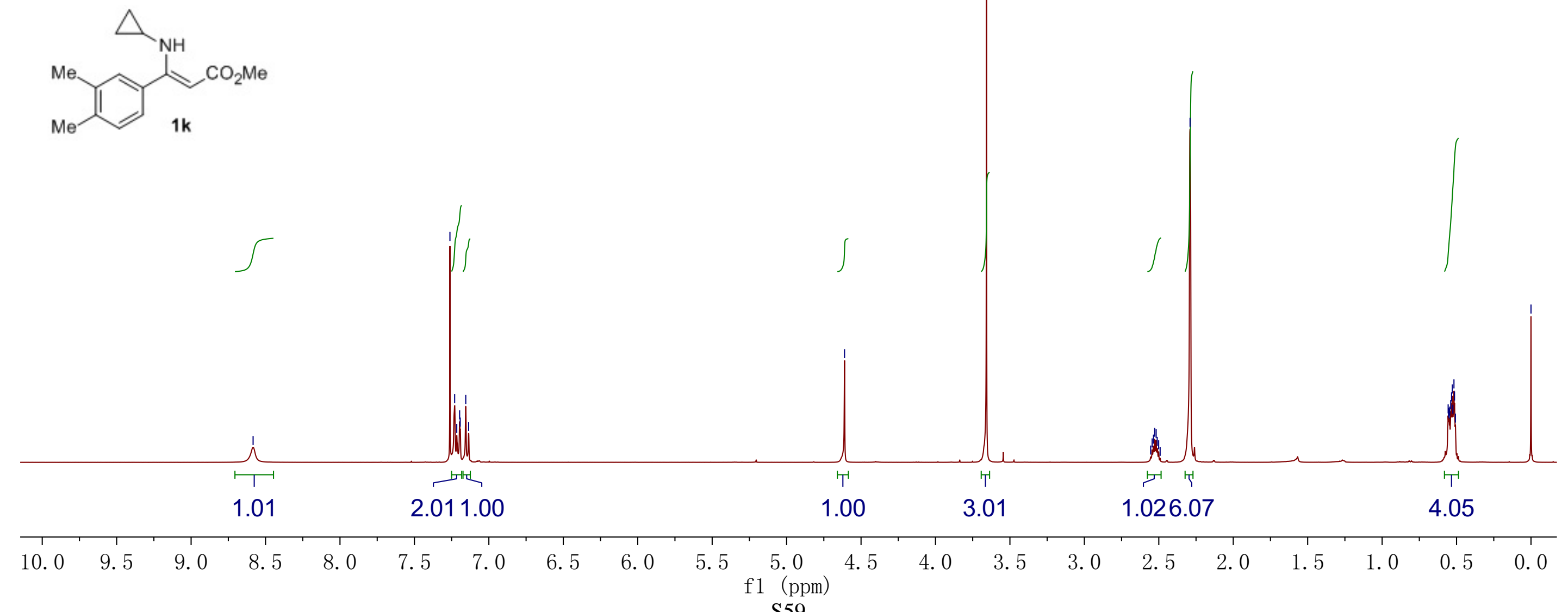
${ }^{13} \mathrm{C}$ NMR $\left(100 \mathrm{MHz}, \mathrm{CDCl}_{3}\right)$
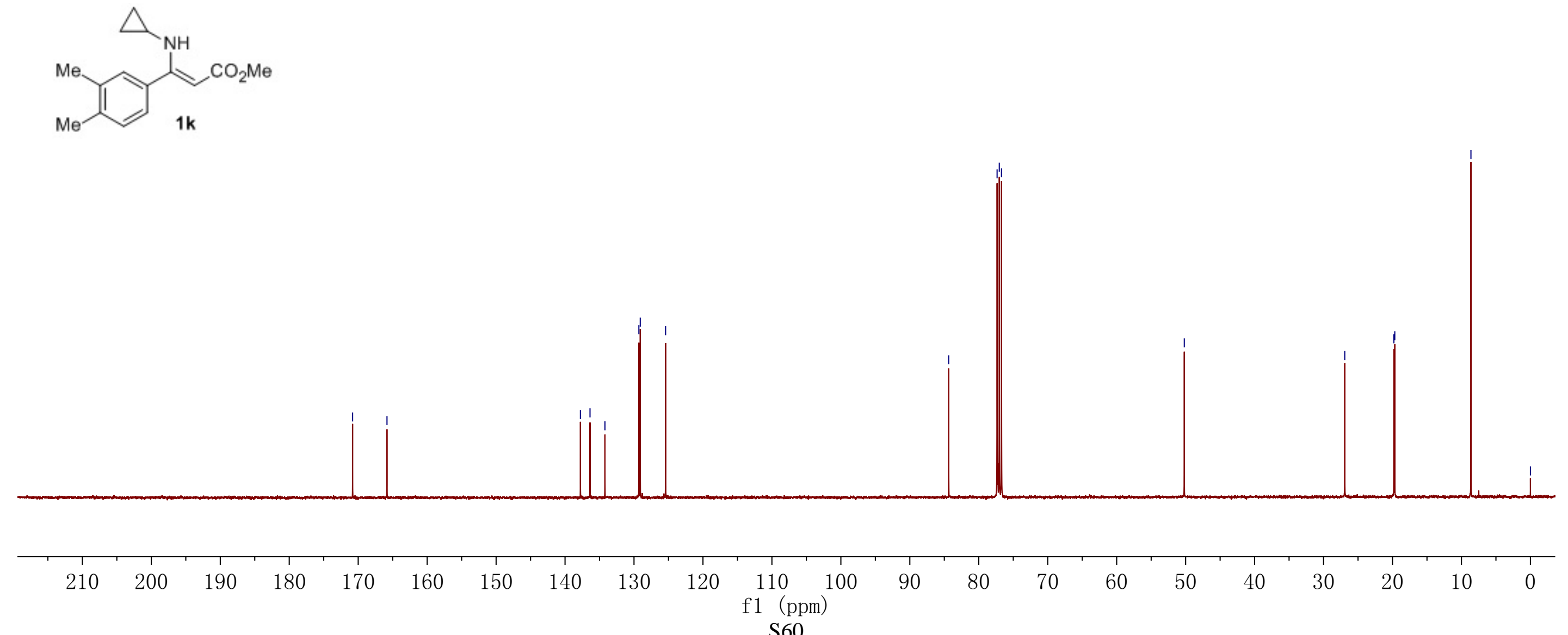


\section{${ }^{1} \mathrm{H} \mathrm{NMR}\left(400 \mathrm{MHz}, \mathrm{CDCl}_{3}\right)$}

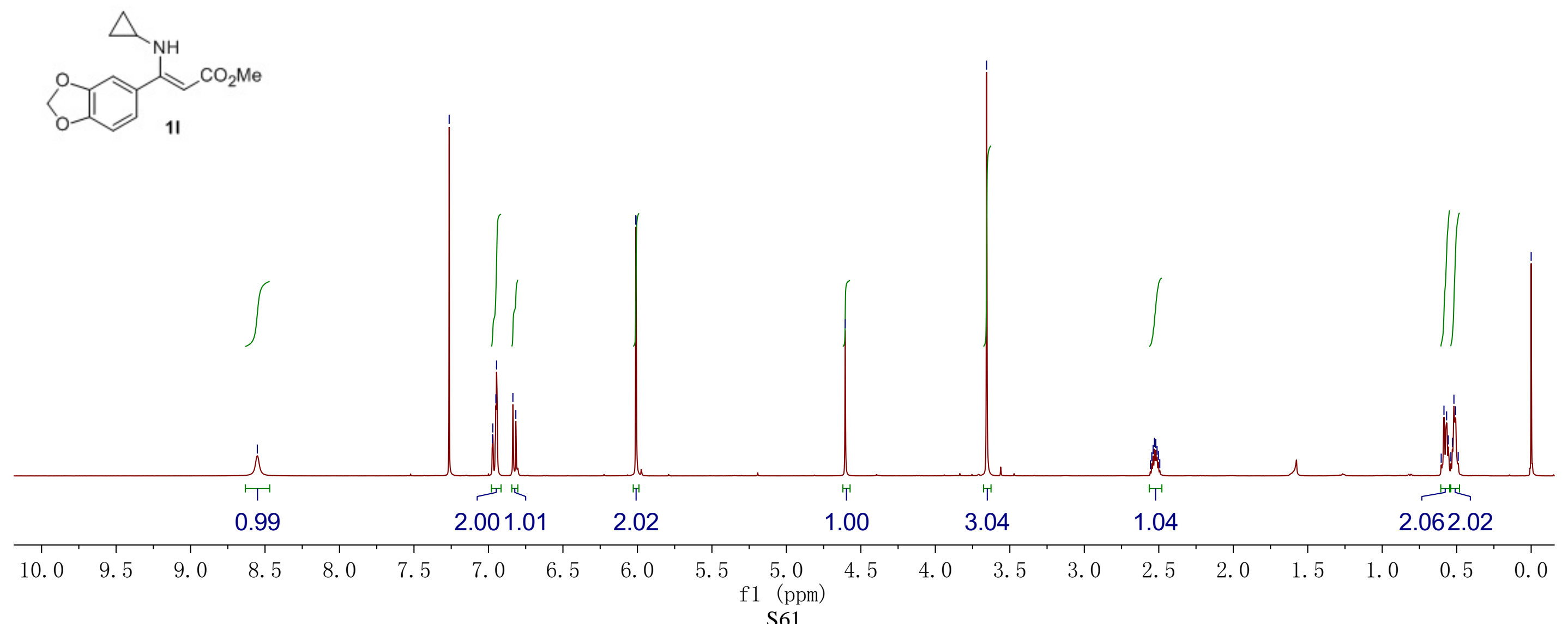




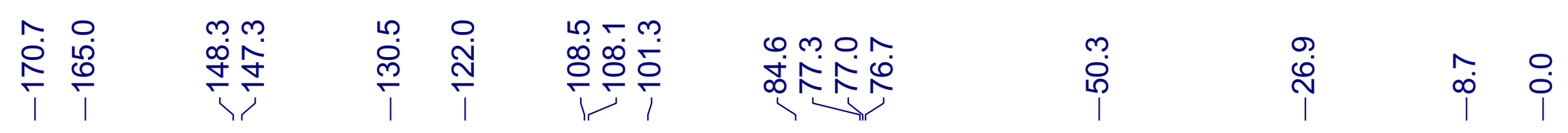

${ }^{13} \mathrm{C} \mathrm{NMR}\left(100 \mathrm{MHz}, \mathrm{CDCl}_{3}\right)$

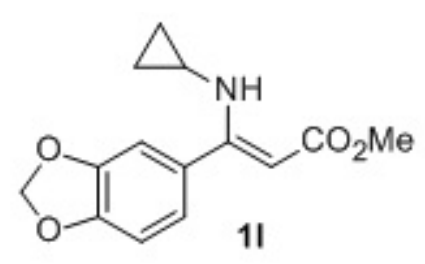


${ }^{1} \mathrm{H}$ NMR (400 MHz, $\left.\mathrm{CDCl}_{3}\right)$

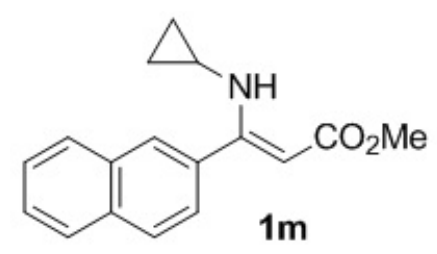

m

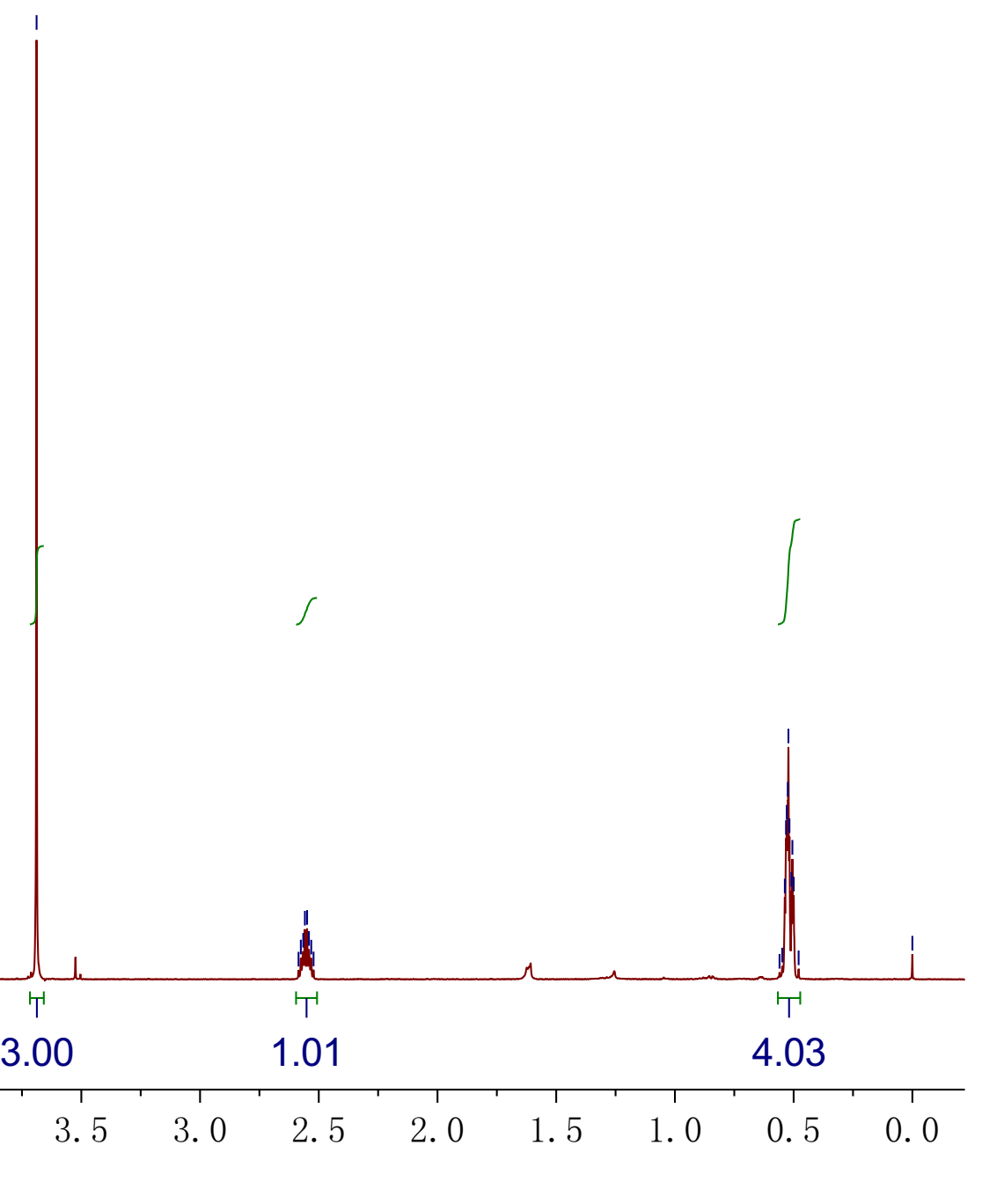


${ }^{13} \mathrm{C}$ NMR (100 MHz, $\left.\mathrm{CDCl}_{3}\right)$

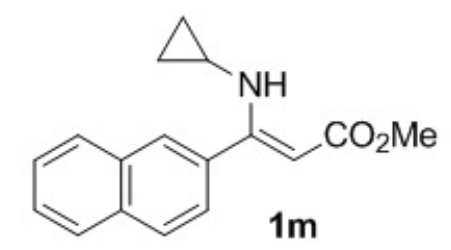

$1 \mathrm{~m}$

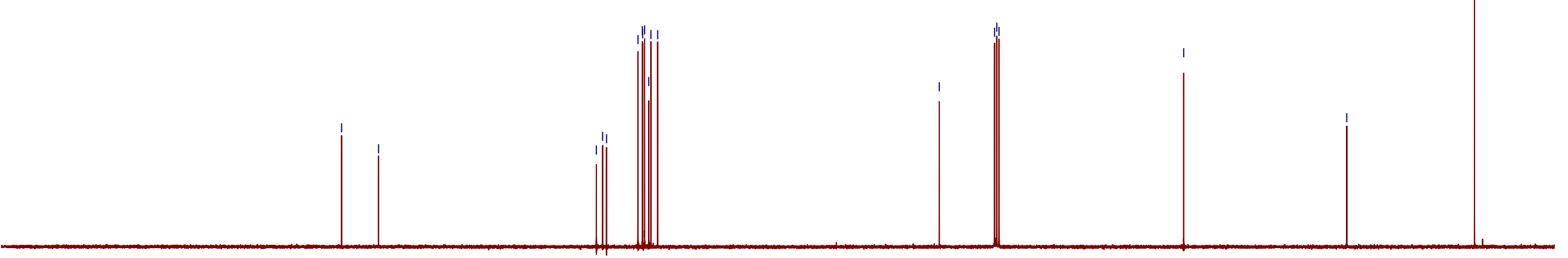


${ }^{1} \mathrm{H}$ NMR (400 MHz, $\mathrm{CDCl}_{3}$ )
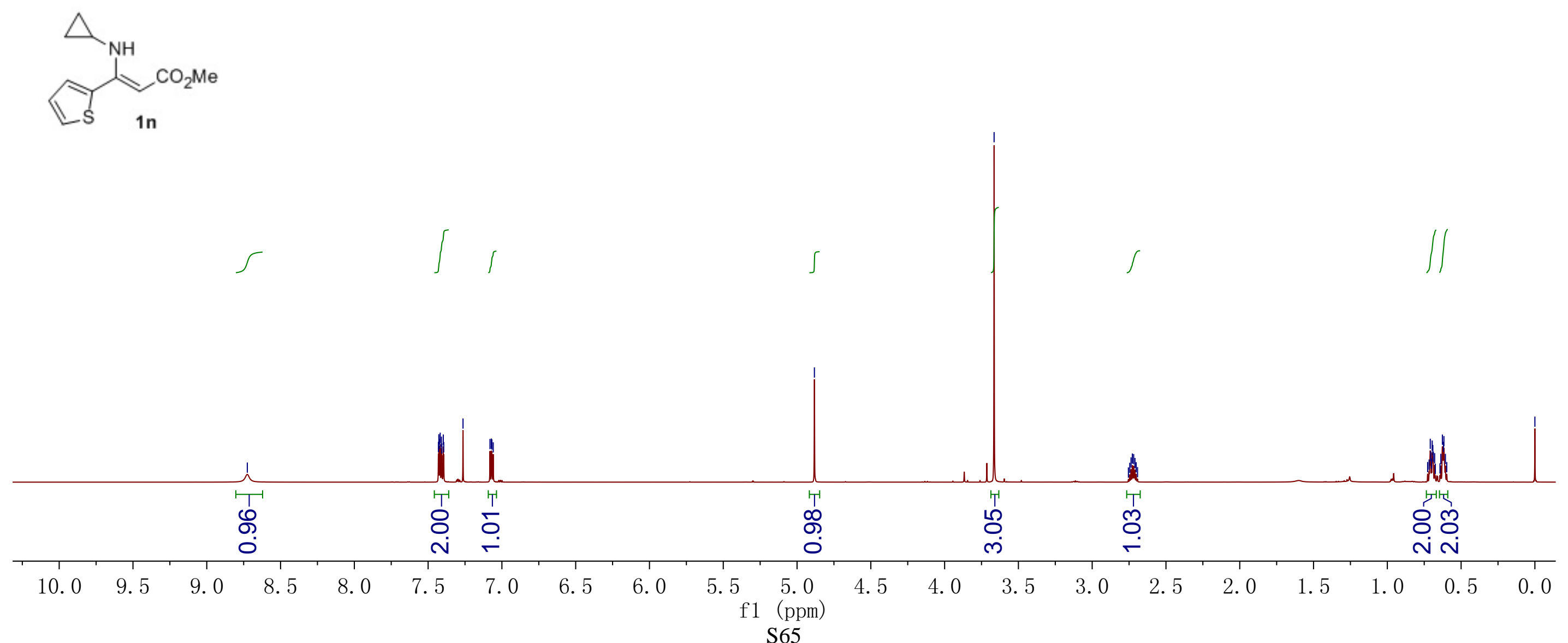


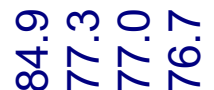

$>$ r

${ }^{13} \mathrm{C}$ NMR $\left(100 \mathrm{MHz}, \mathrm{CDCl}_{3}\right)$

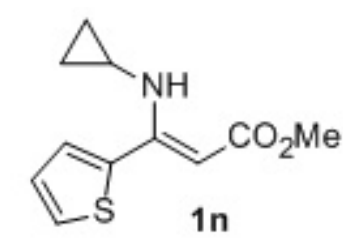

1n

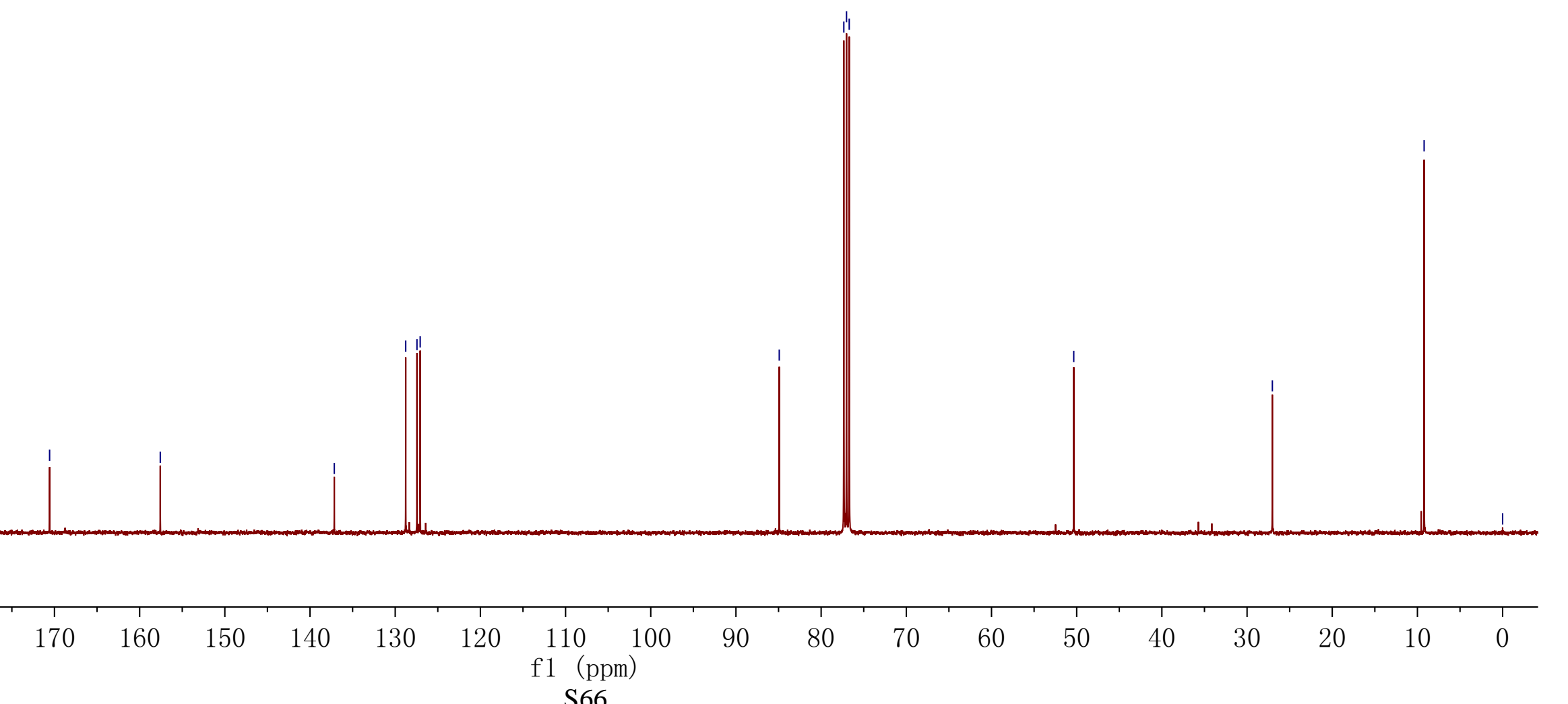


○)

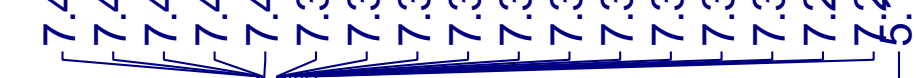

।

${ }^{1} \mathrm{H}$ NMR (400 MHz, $\mathrm{CDCl}_{3}$ )

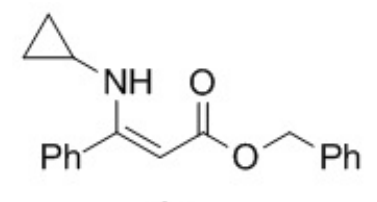

10

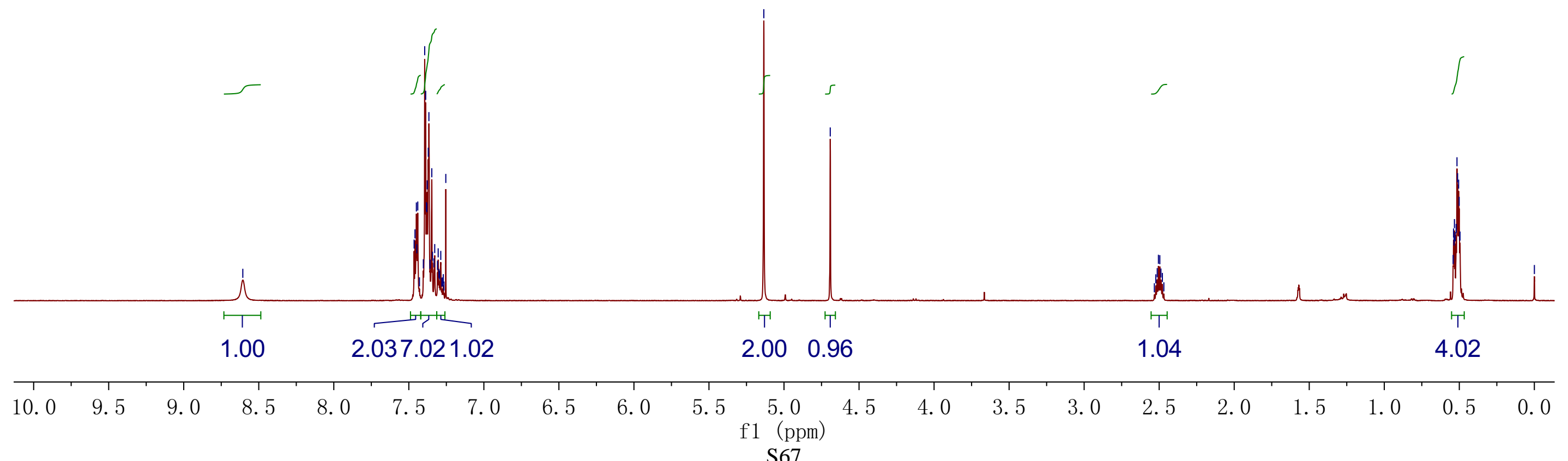


${ }^{13} \mathrm{C} \mathrm{NMR}\left(100 \mathrm{MHz}, \mathrm{CDCl}_{3}\right)$

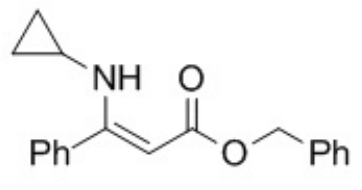

10

$$
\text { (1) }
$$


${ }^{1} \mathrm{H} \mathrm{NMR}\left(400 \mathrm{MHz}, \mathrm{CDCl}_{3}\right)$

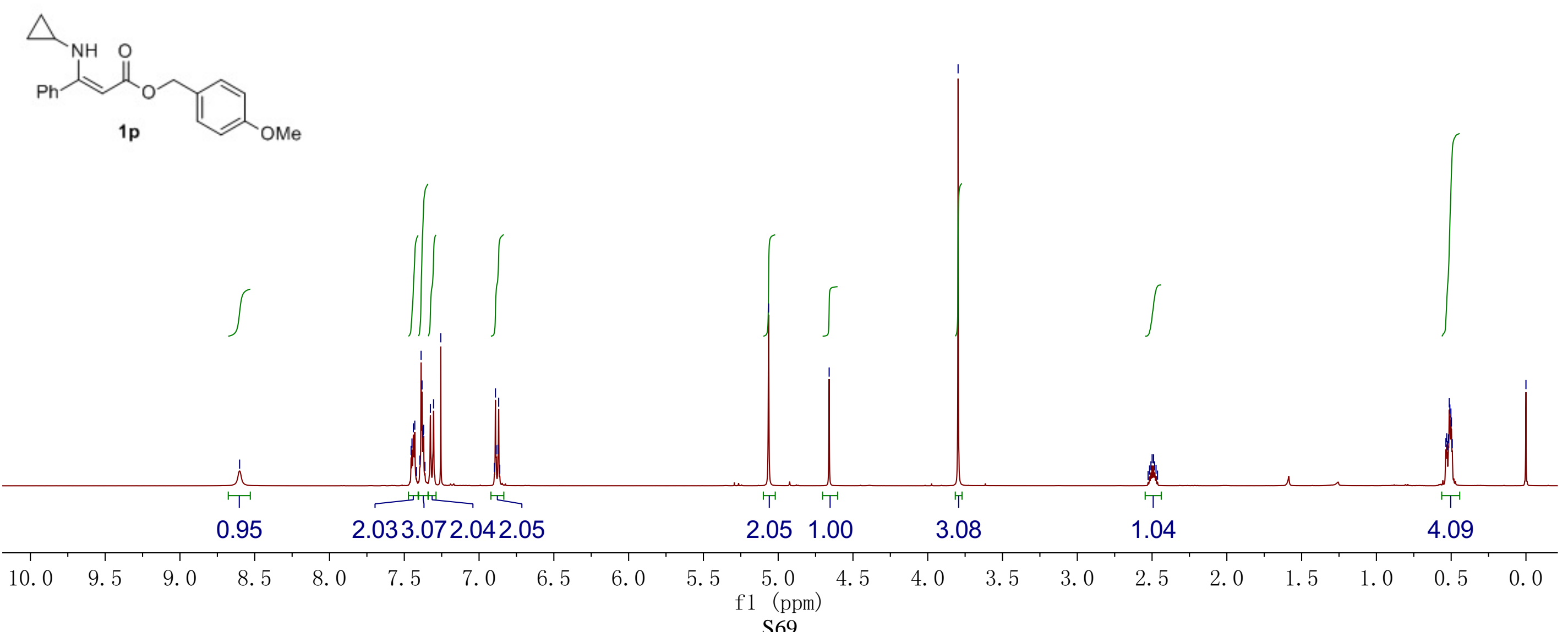


${ }^{13} \mathrm{C} \mathrm{NMR}\left(100 \mathrm{MHz}, \mathrm{CDCl}_{3}\right)$
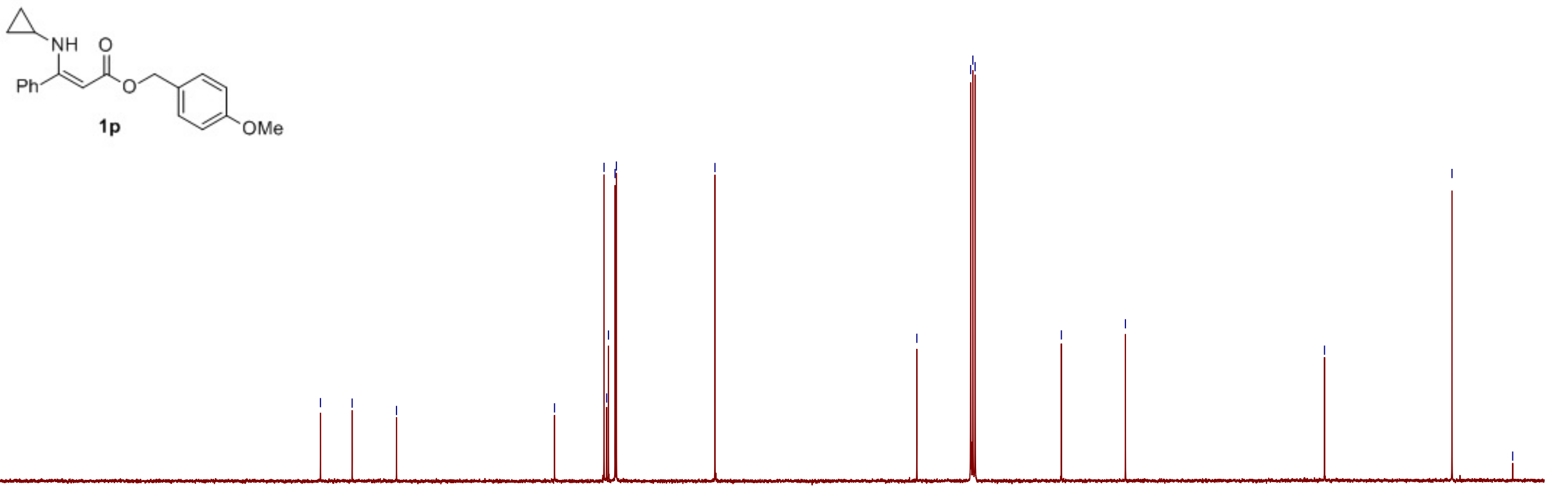

210

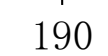


${ }^{1} \mathrm{H}$ NMR (400 MHz, $\mathrm{CDCl}_{3}$ )
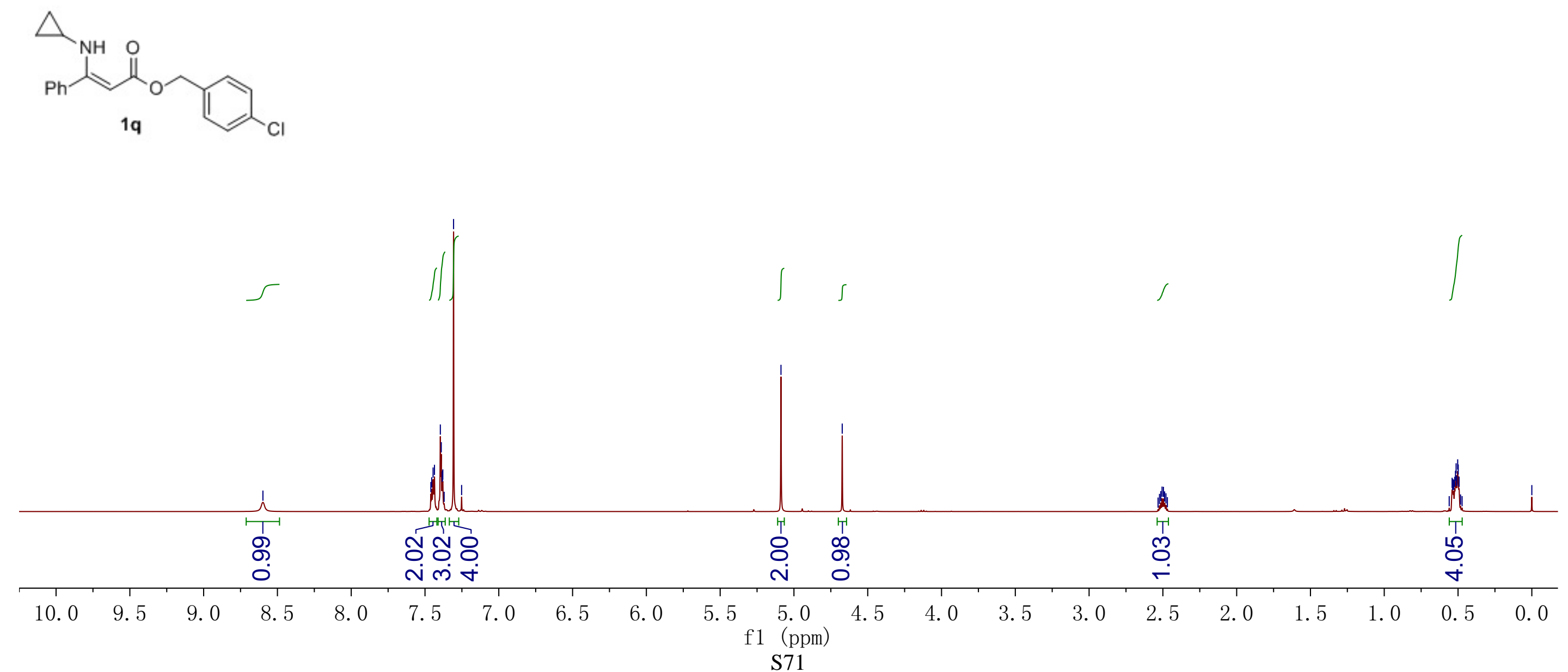
${ }^{13} \mathrm{C}$ NMR $\left(100 \mathrm{MHz}, \mathrm{CDCl}_{3}\right)$

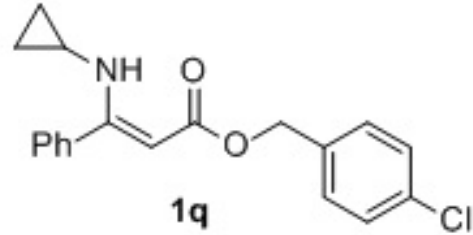

$\mathrm{Cl}_{\mathrm{Cl}}$

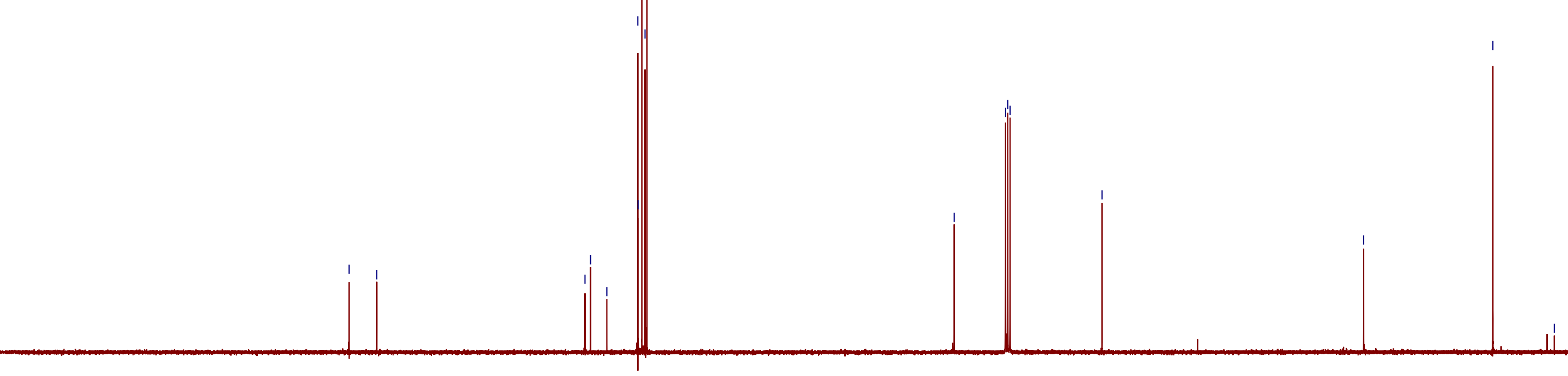


${ }^{1} \mathrm{H}$ NMR (400 MHz, $\mathrm{CDCl}_{3}$ )

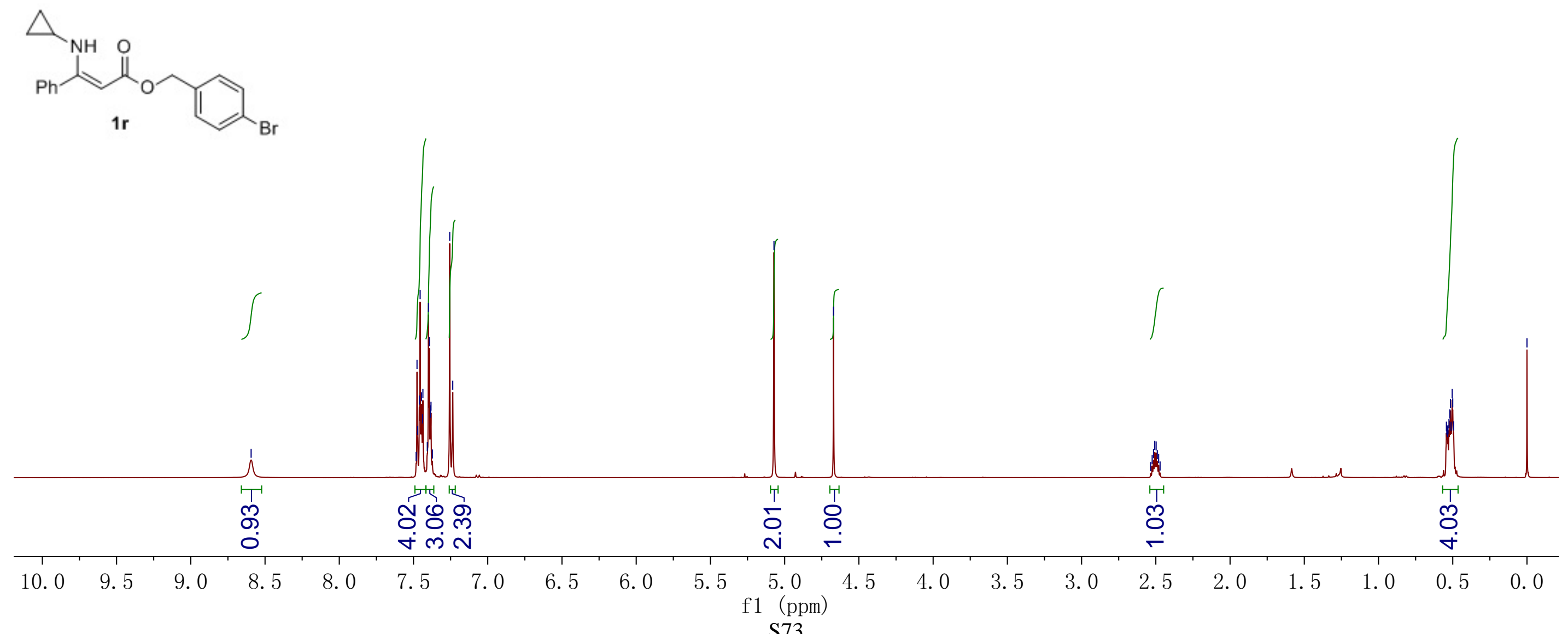


${ }^{13} \mathrm{C}$ NMR $\left(100 \mathrm{MHz}, \mathrm{CDCl}_{3}\right)$
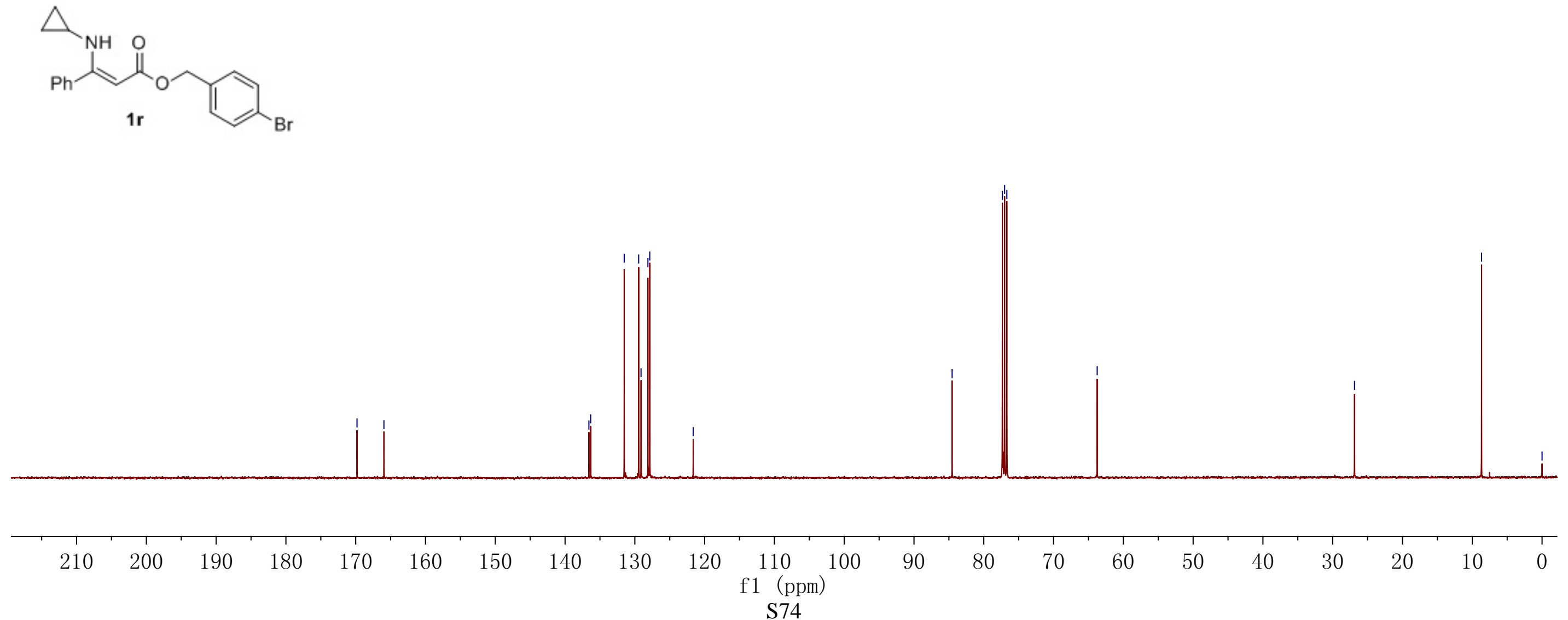
${ }^{1} \mathrm{H} \mathrm{NMR}\left(400 \mathrm{MHz}, \mathrm{CDCl}_{3}\right)$

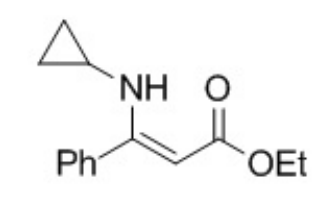

1s

$$
\text { OEt }
$$

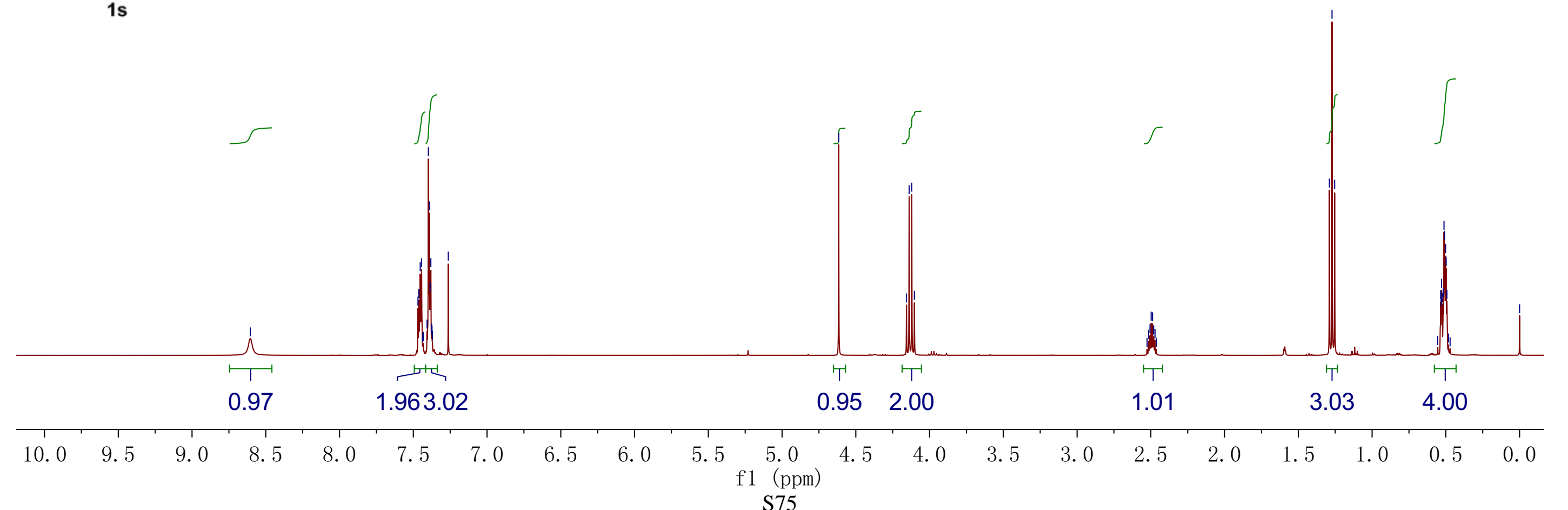


${ }^{13} \mathrm{C}$ NMR (100 MHz, $\left.\mathrm{CDCl}_{3}\right)$
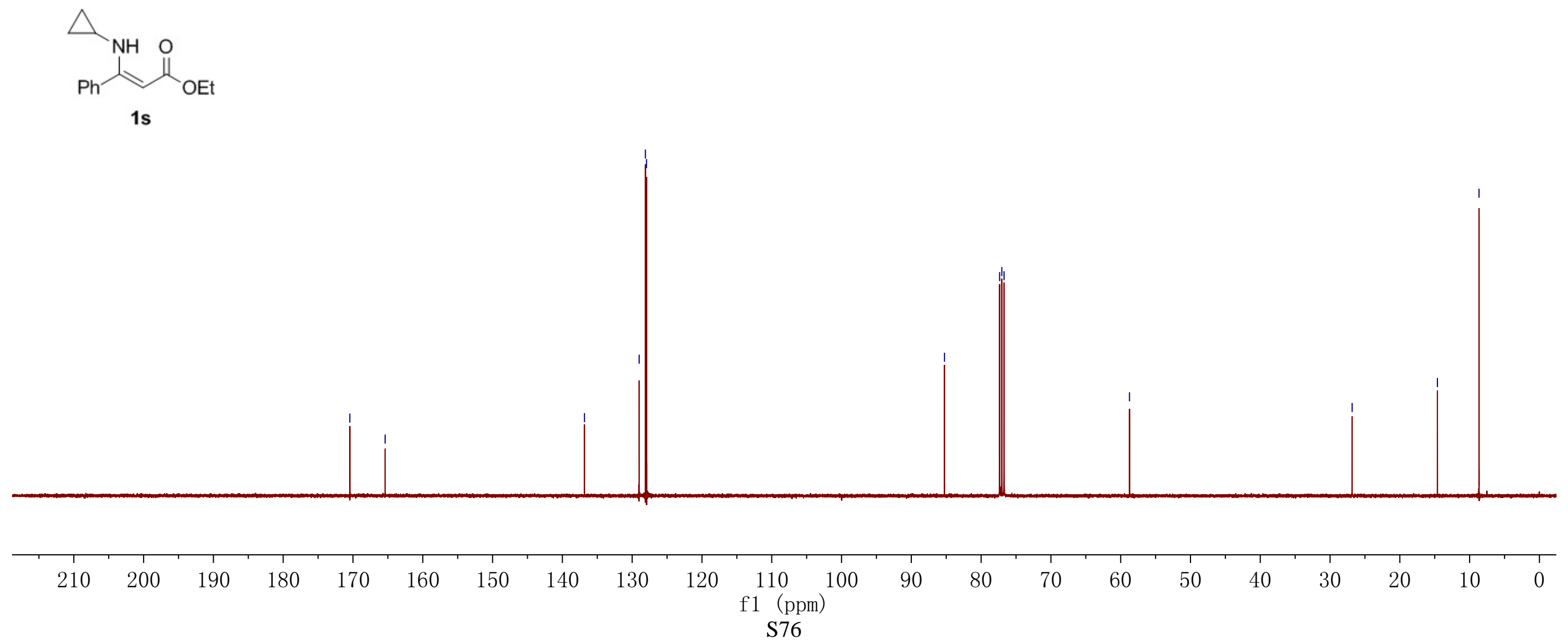
${ }^{1} \mathrm{H} \mathrm{NMR}\left(400 \mathrm{MHz}, \mathrm{CDCl}_{3}\right)$
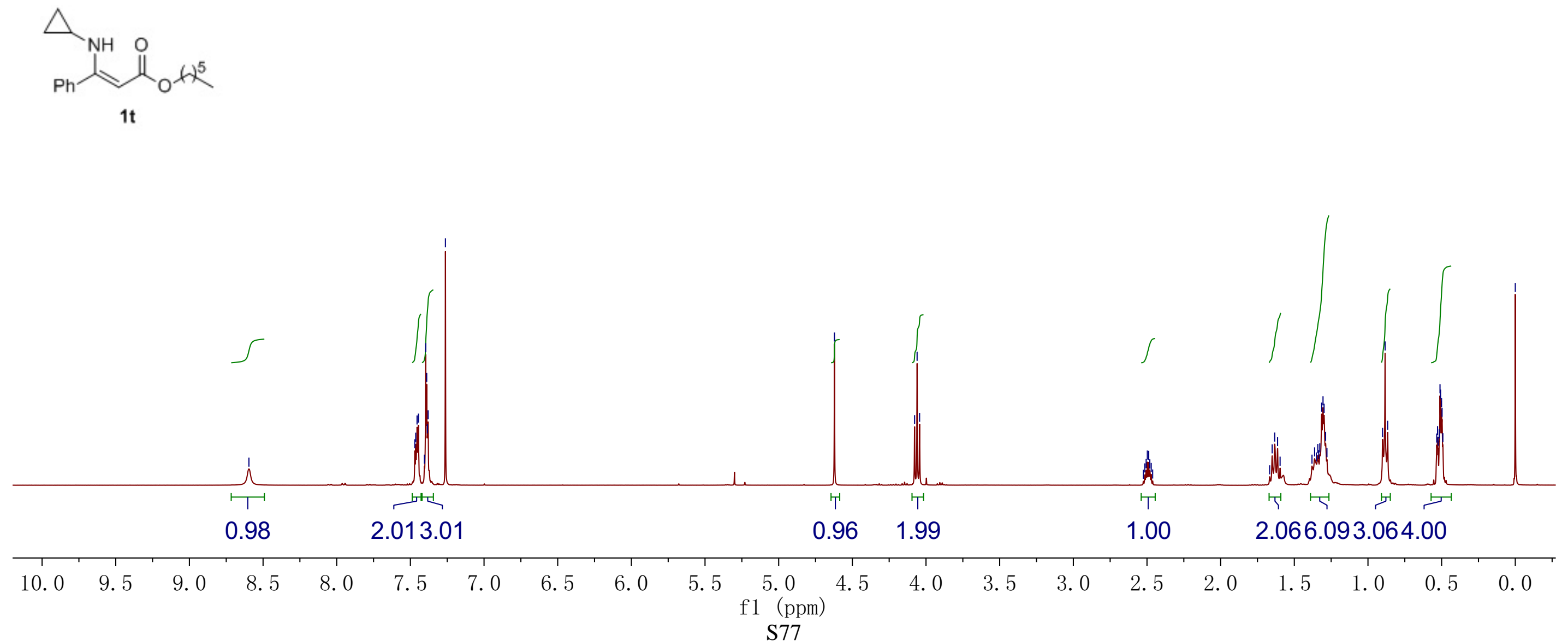
${ }^{13} \mathrm{C} \mathrm{NMR}\left(100 \mathrm{MHz}, \mathrm{CDCl}_{3}\right)$

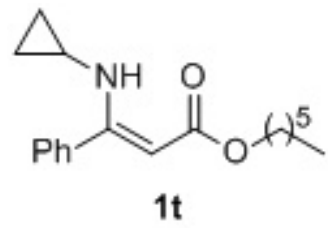




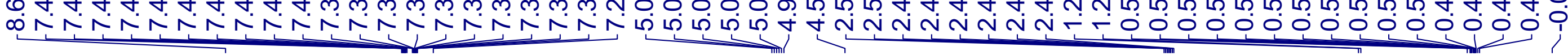

${ }^{1} \mathrm{H} \mathrm{NMR}\left(400 \mathrm{MHz}, \mathrm{CDCl}_{3}\right)$
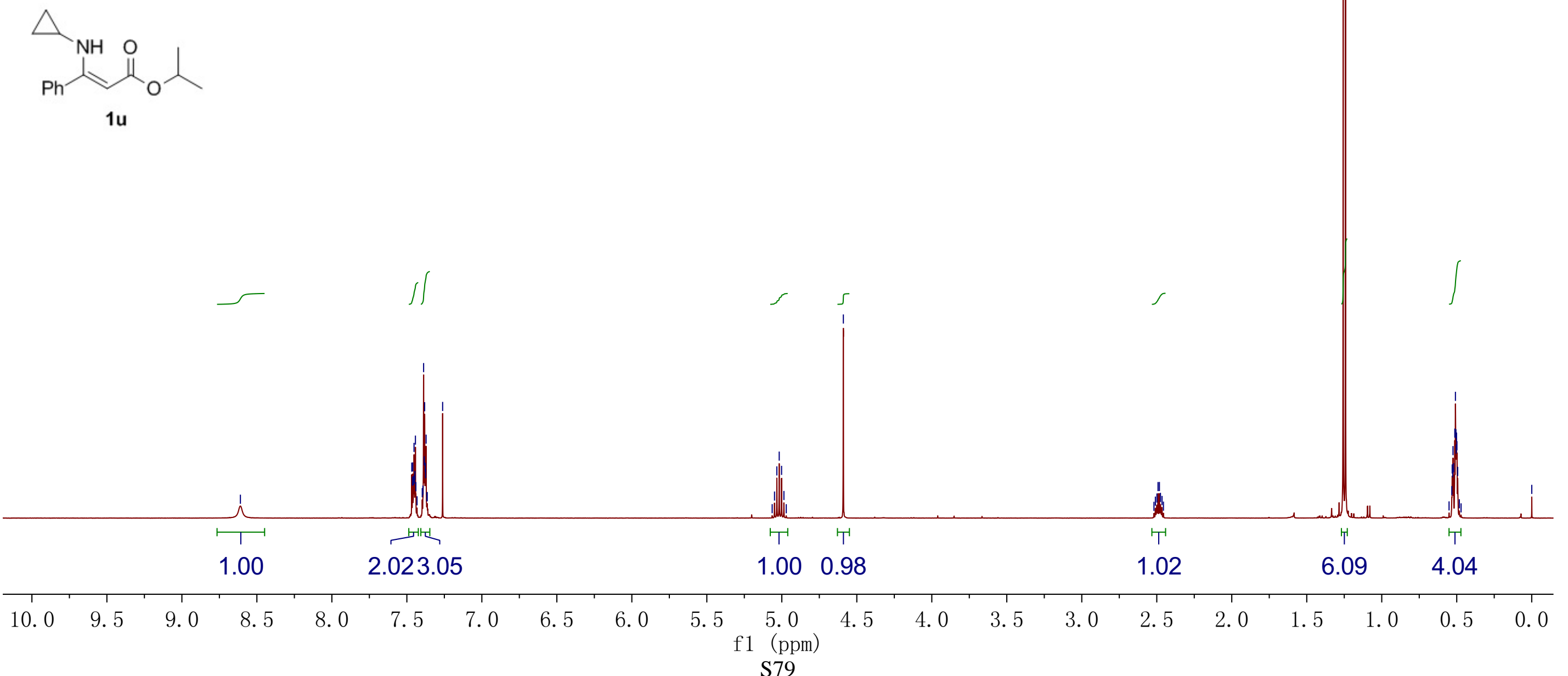


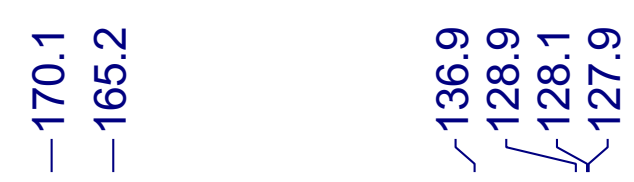

ڤ

$\stackrel{\infty}{\infty} \underset{1}{ } \quad \infty$

${ }^{13} \mathrm{C}$ NMR (100 MHz, $\left.\mathrm{CDCl}_{3}\right)$
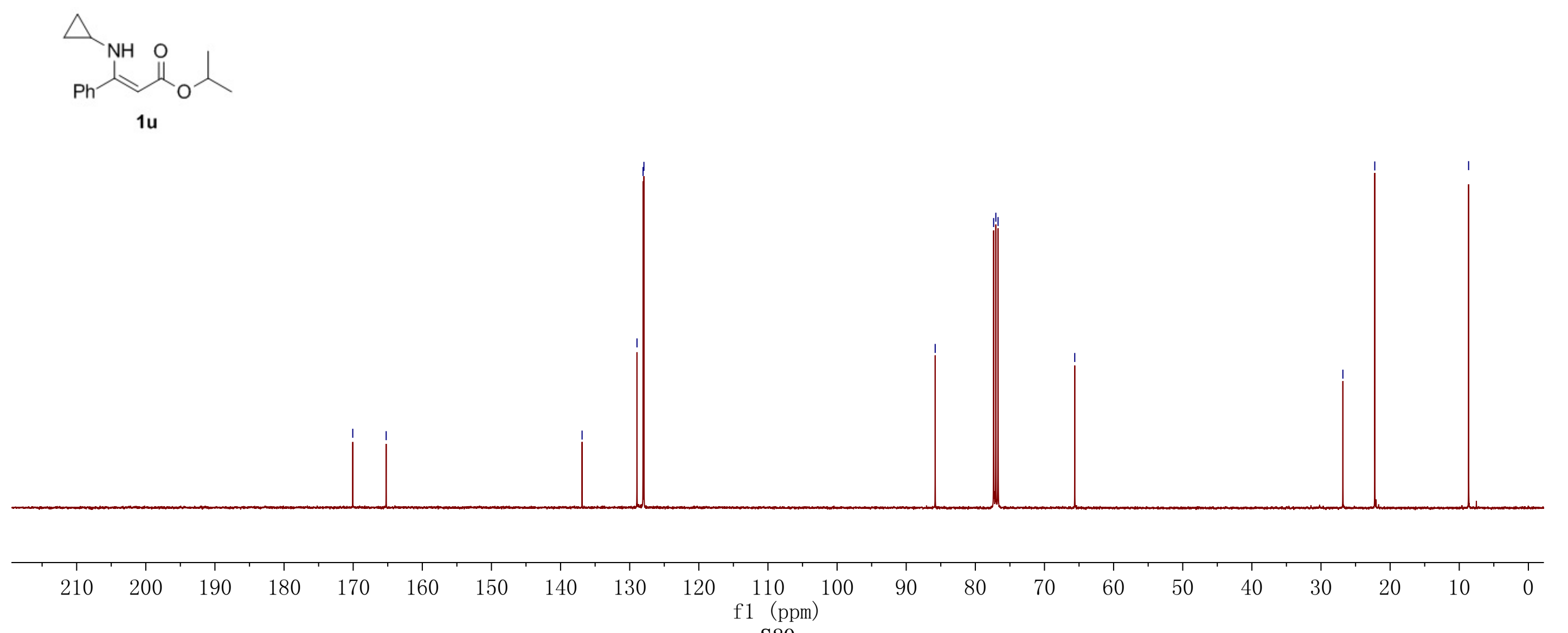


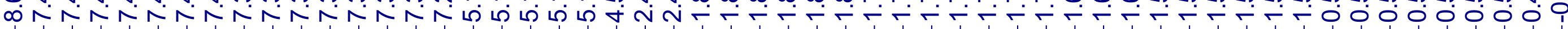

${ }^{1} \mathrm{H} \mathrm{NMR}\left(400 \mathrm{MHz}, \mathrm{CDCl}_{3}\right)$
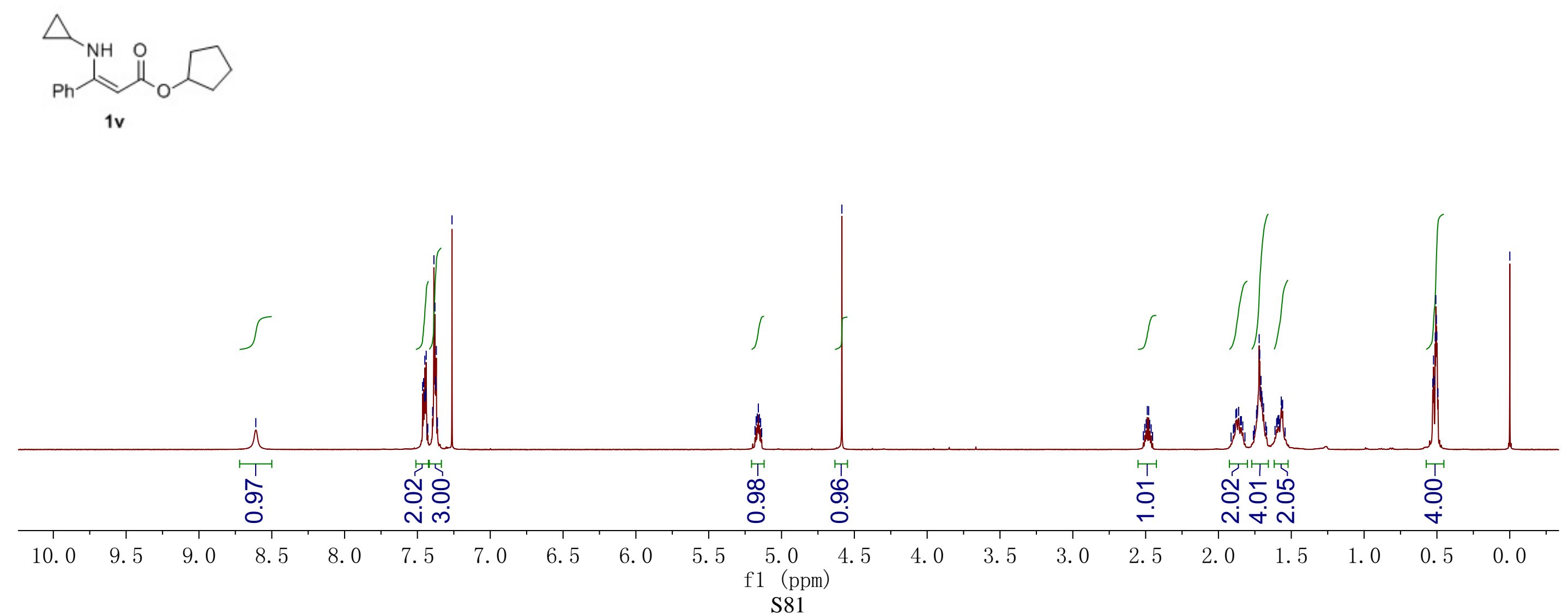


\section{${ }^{13} \mathrm{C}$ NMR (100 MHz, $\left.\mathrm{CDCl}_{3}\right)$}
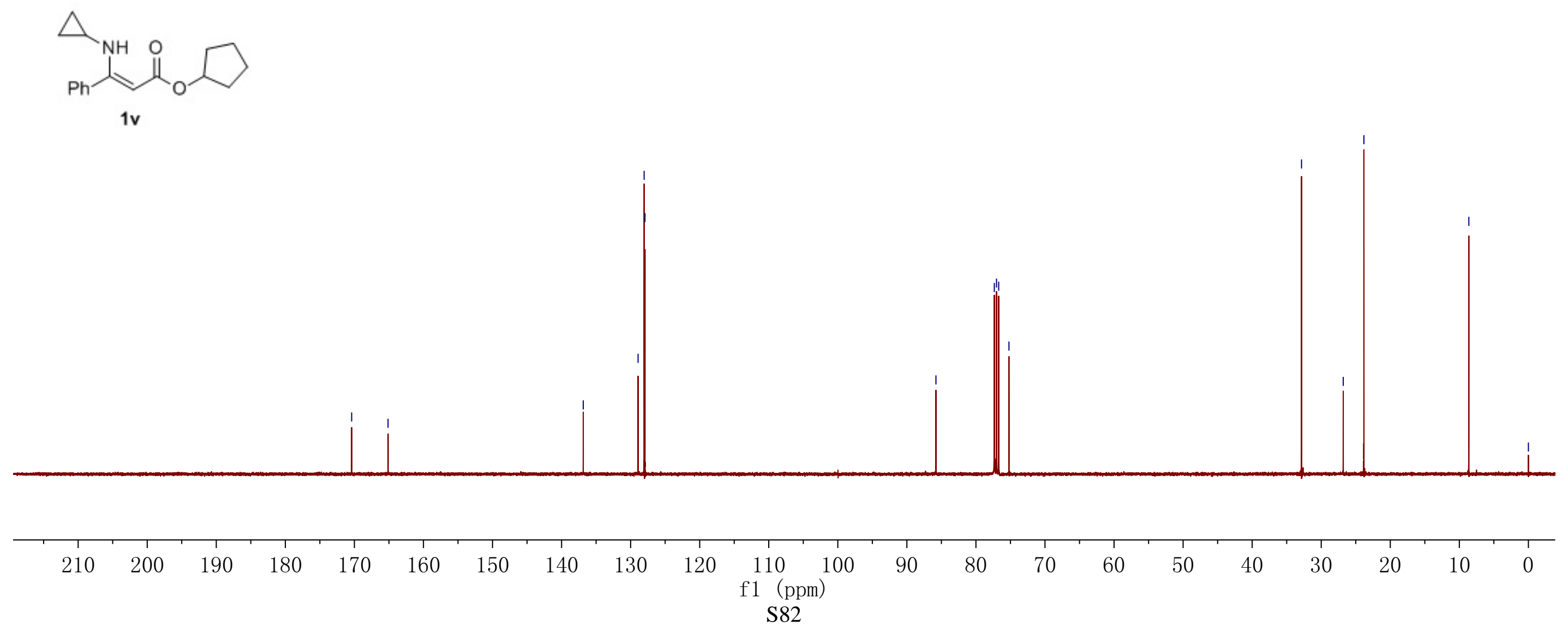
${ }^{1} \mathrm{H}$ NMR (400 MHz, $\mathrm{CDCl}_{3}$ )

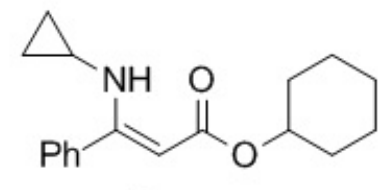

$1 w$

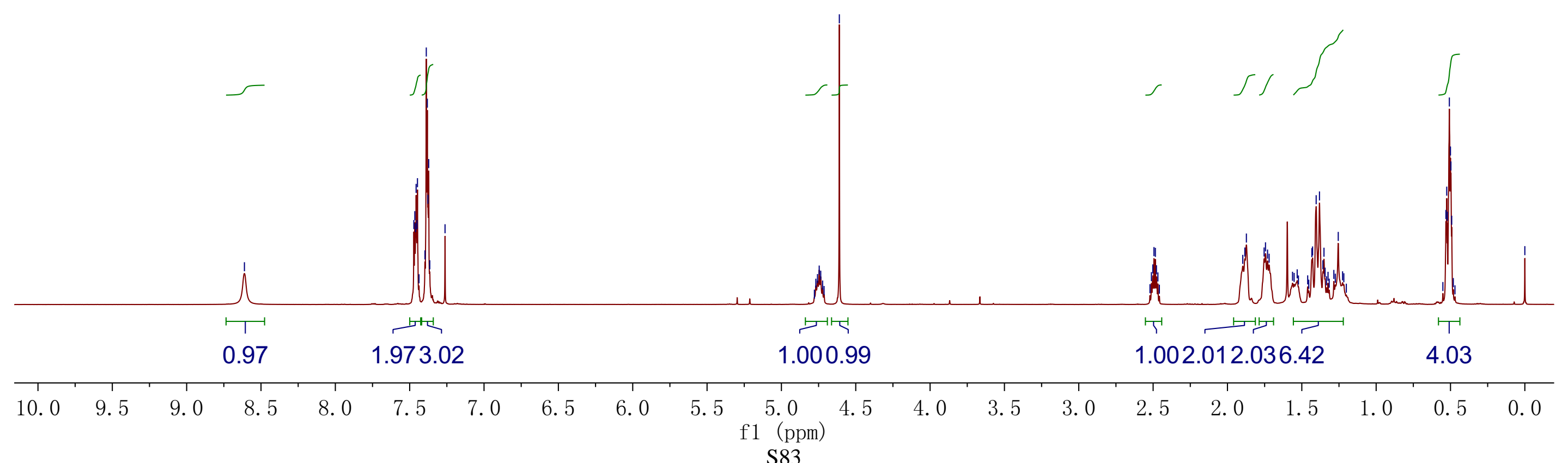


${ }^{13} \mathrm{C}$ NMR $\left(100 \mathrm{MHz}, \mathrm{CDCl}_{3}\right)$

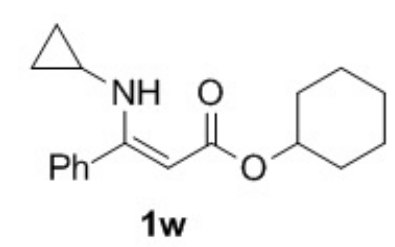

$1 w$

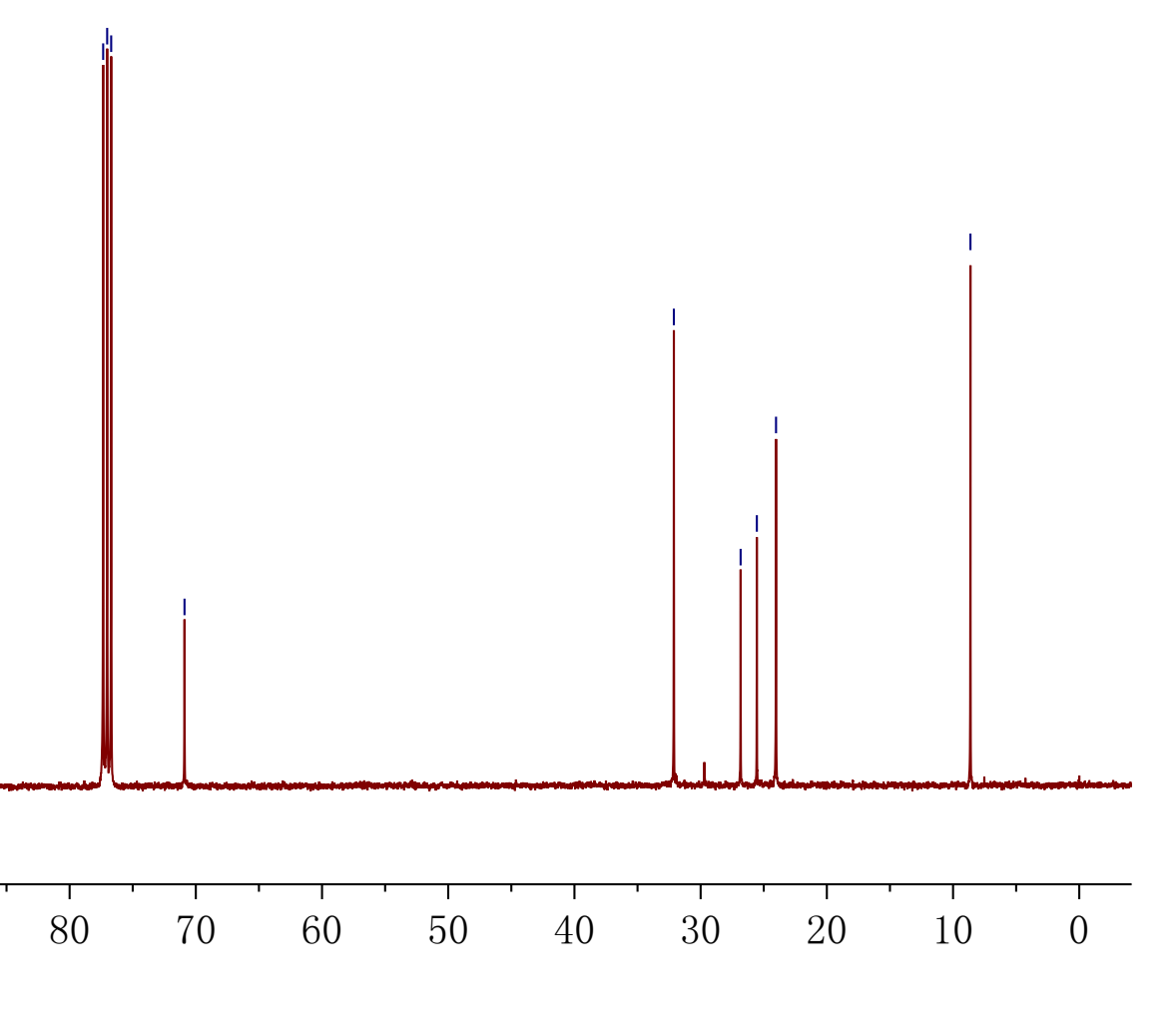


${ }^{1} \mathrm{H}$ NMR (400 MHz, $\mathrm{CDCl}_{3}$ )
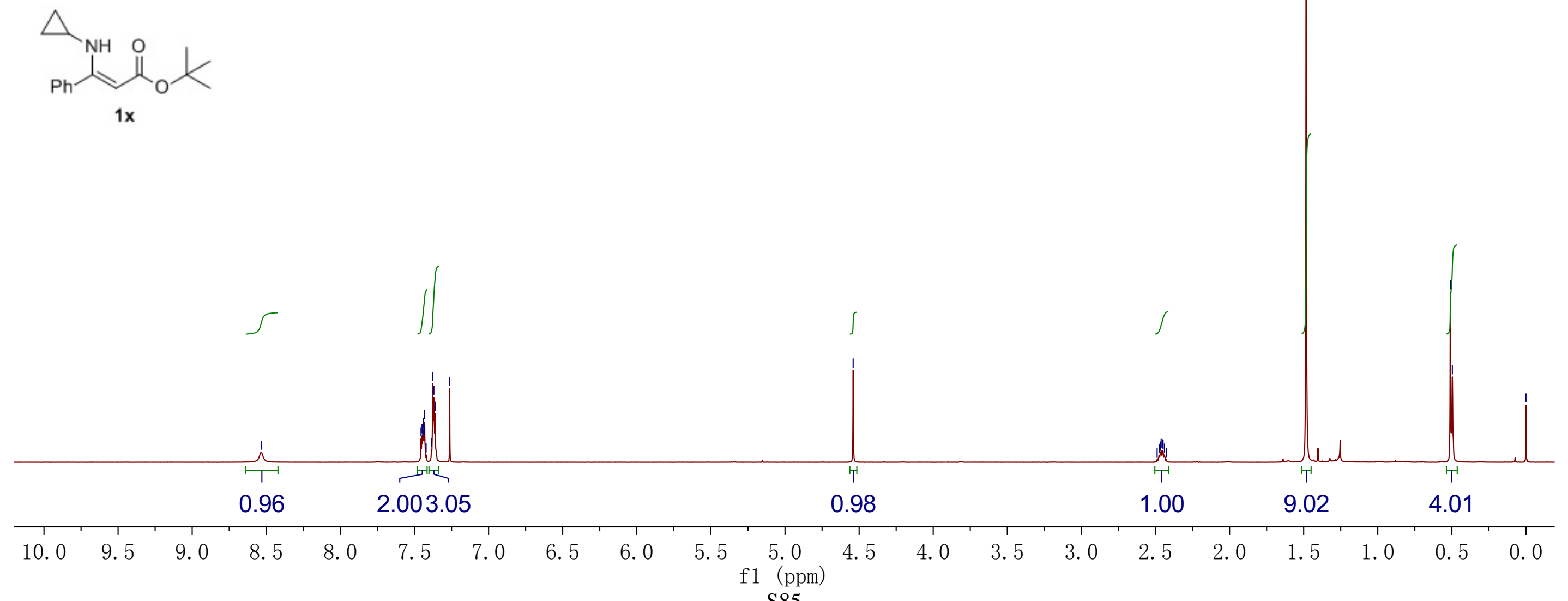


\section{${ }^{13} \mathrm{C}$ NMR (100 MHz, $\left.\mathrm{CDCl}_{3}\right)$}
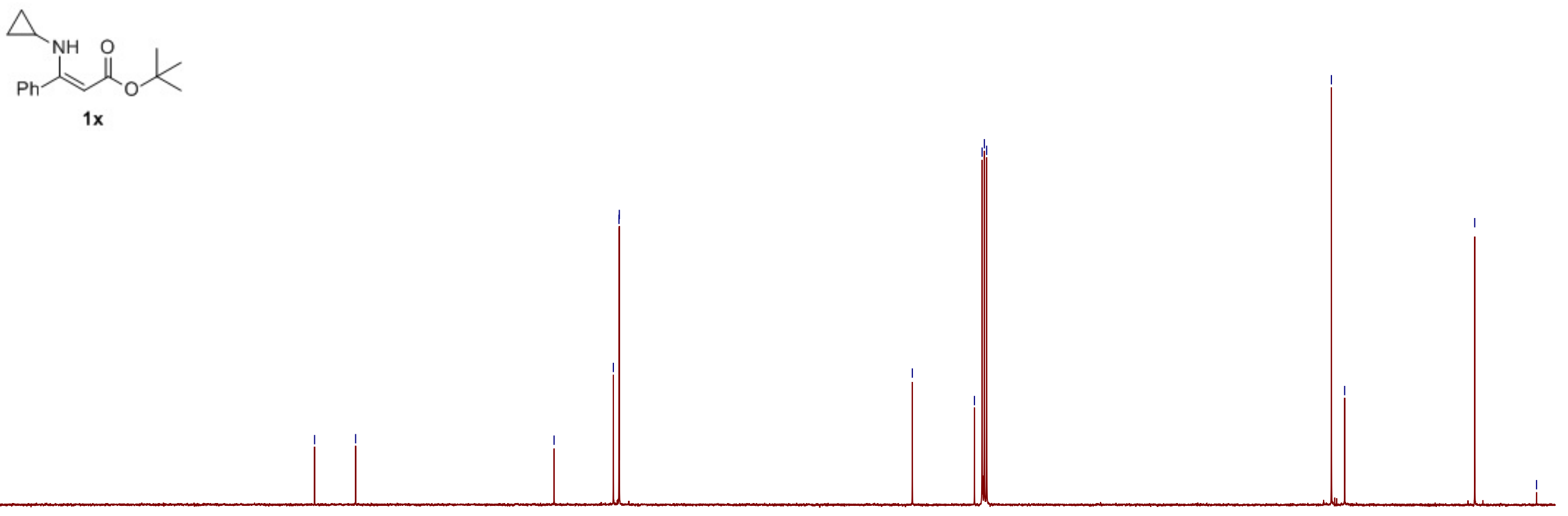


\section{${ }^{1} \mathrm{H}$ NMR (400 MHz, $\mathrm{CDCl}_{3}$ )}
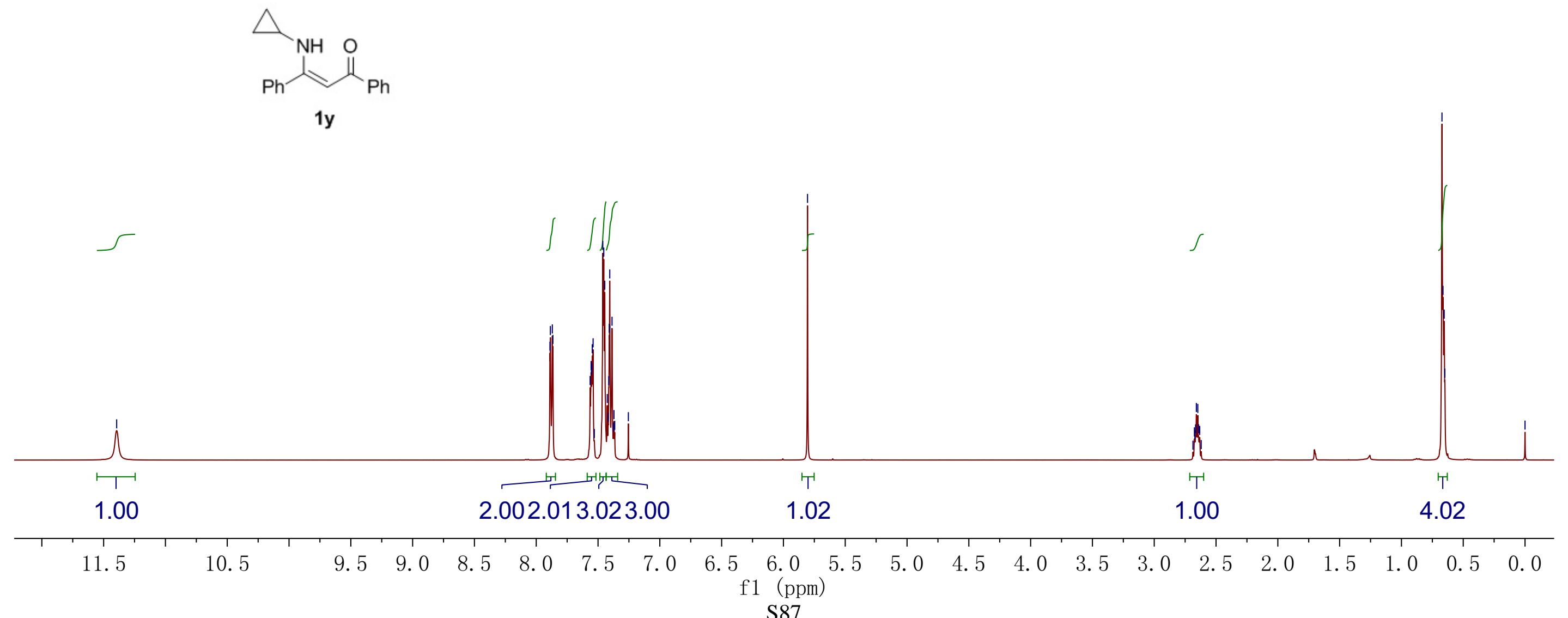

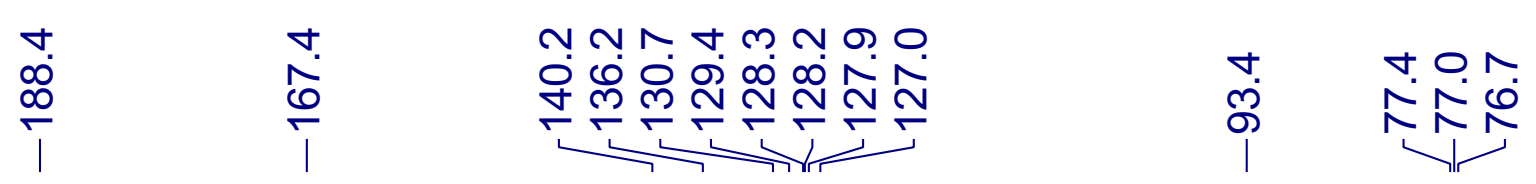

${ }^{13} \mathrm{C} \mathrm{NMR}\left(100 \mathrm{MHz}, \mathrm{CDCl}_{3}\right)$
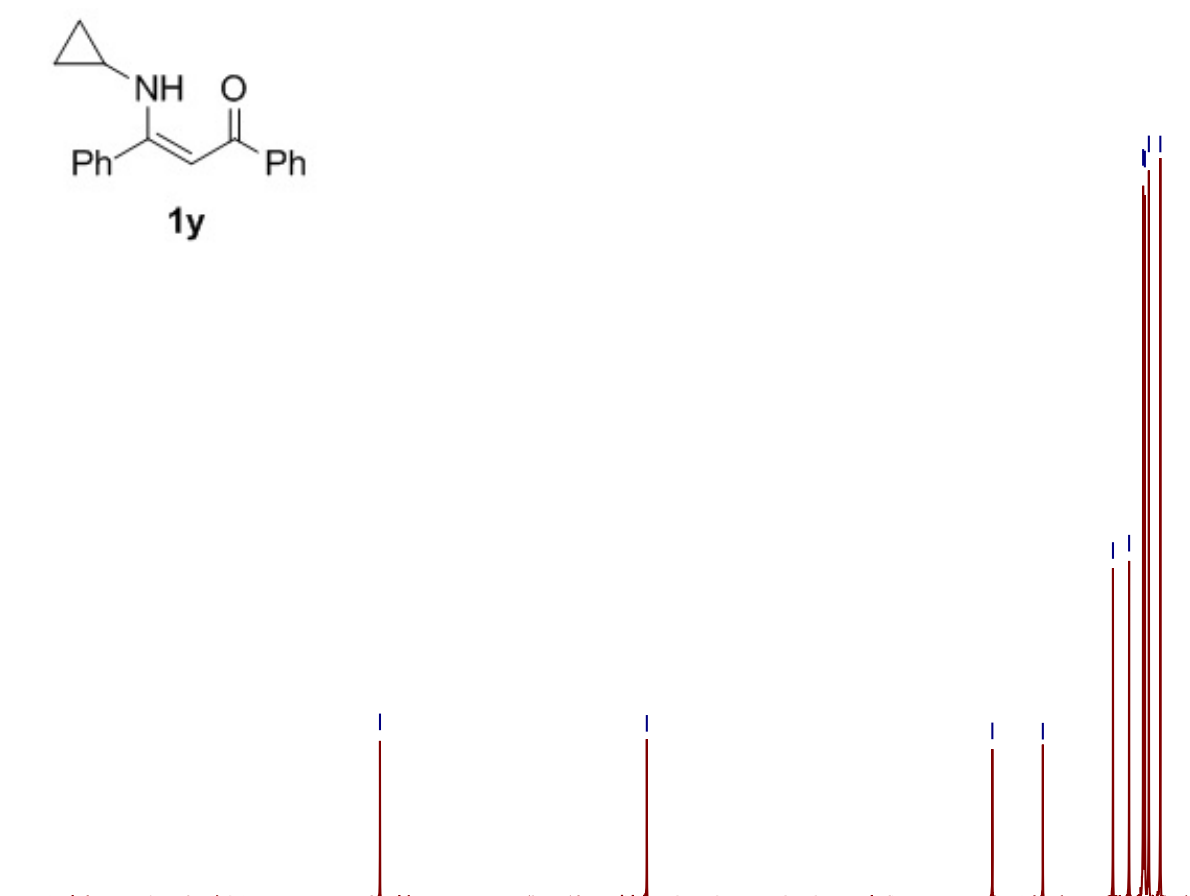

$\begin{array}{lll}1 & & \\ 210 & 200 & 190\end{array}$

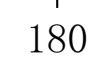

170

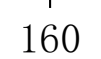

150

140

130

120

110

f1 (ppm) 
${ }^{1} \mathrm{H} \mathrm{NMR}\left(400 \mathrm{MHz}, \mathrm{CDCl}_{3}\right)$

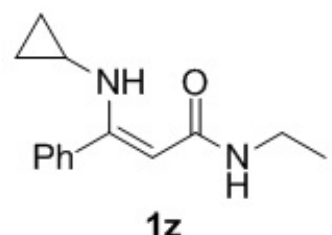

$1 z$

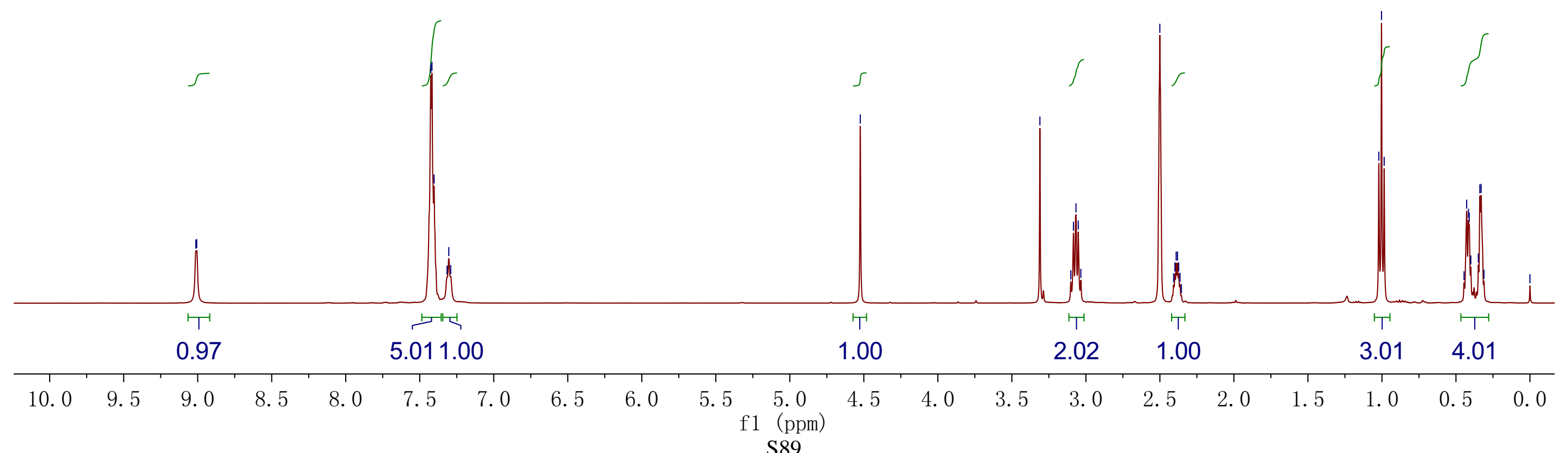




\section{${ }^{13} \mathrm{C}$ NMR (100 MHz, $\left.\mathrm{CDCl}_{3}\right)$}
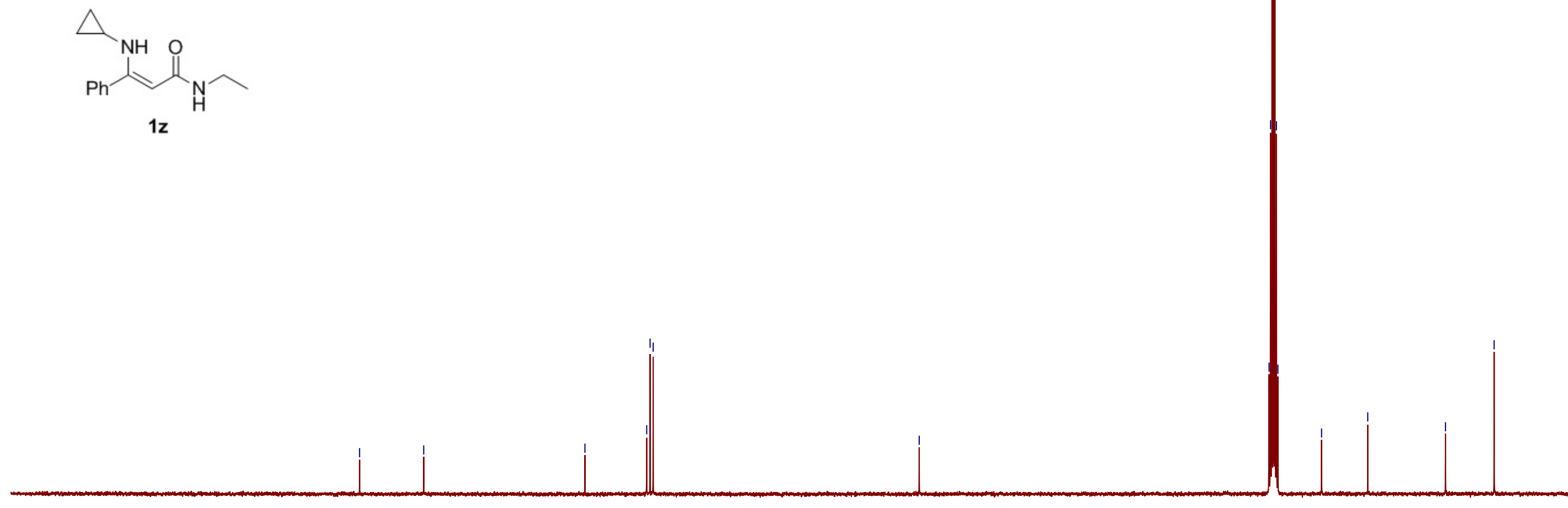
${ }^{1} \mathrm{H}$ NMR (400 MHz, $\mathrm{CDCl}_{3}$ )

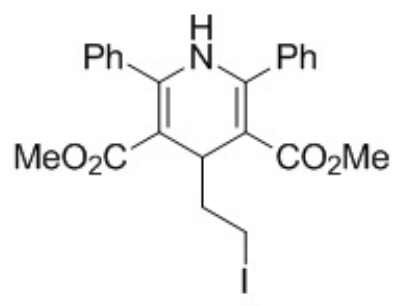

2a

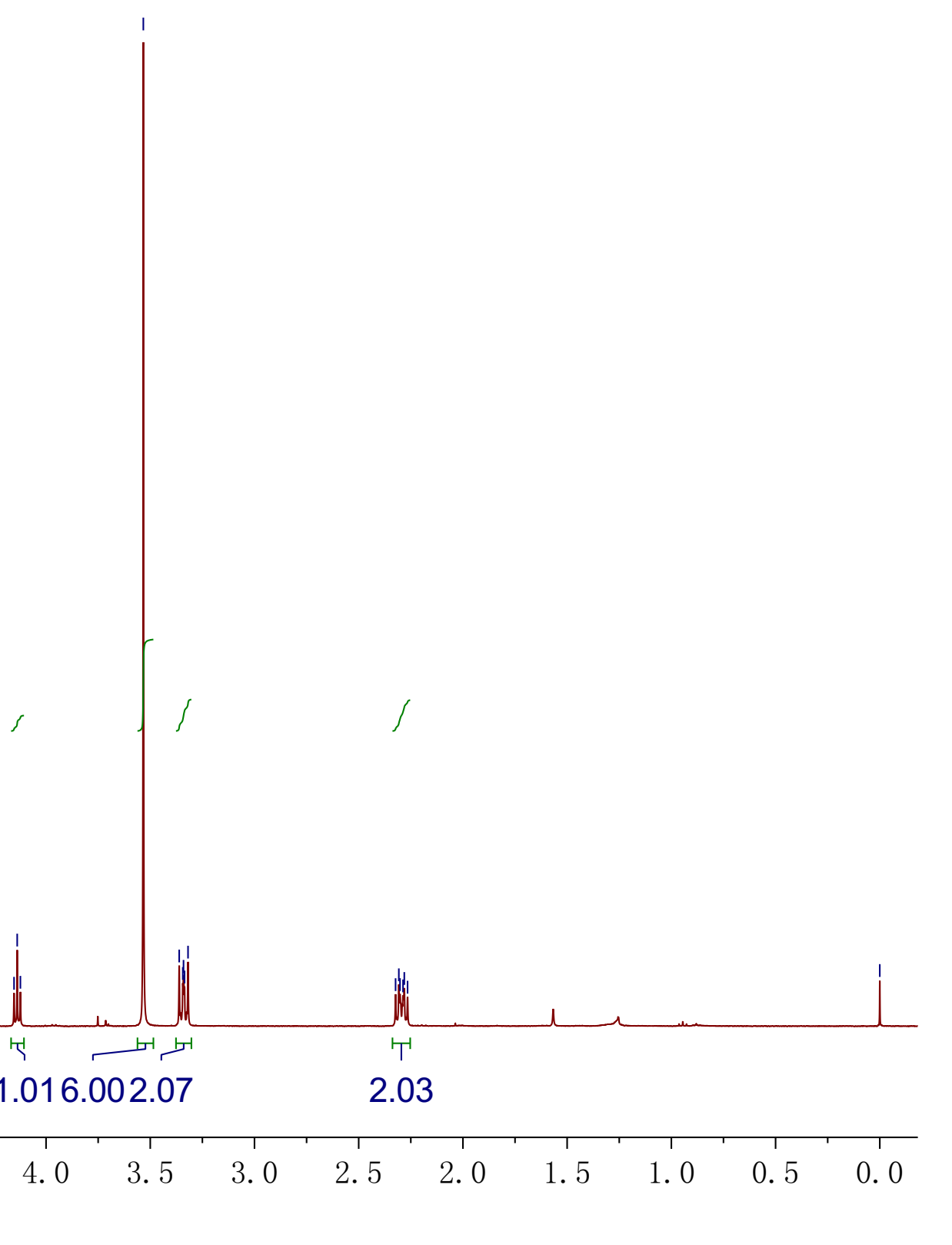


${ }^{13} \mathrm{C} \mathrm{NMR}\left(100 \mathrm{MHz}, \mathrm{CDCl}_{3}\right)$

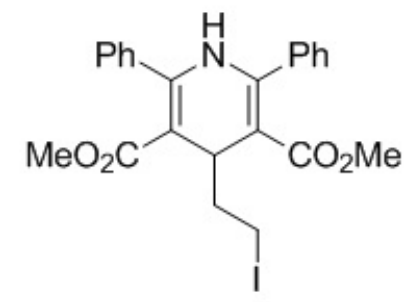

2a

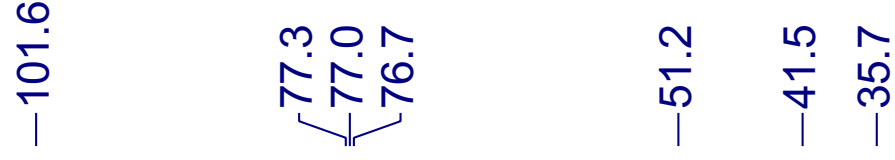

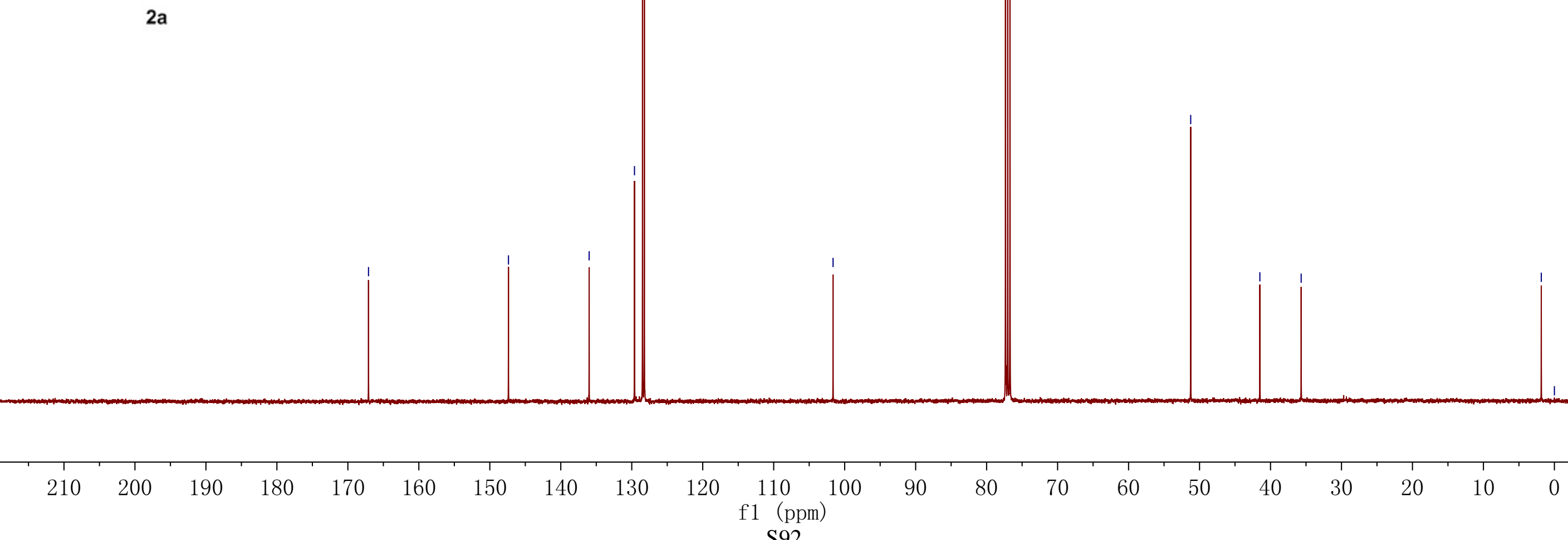


${ }^{1} \mathrm{H}$ NMR (400 MHz, $\left.\mathrm{CDCl}_{3}\right)$

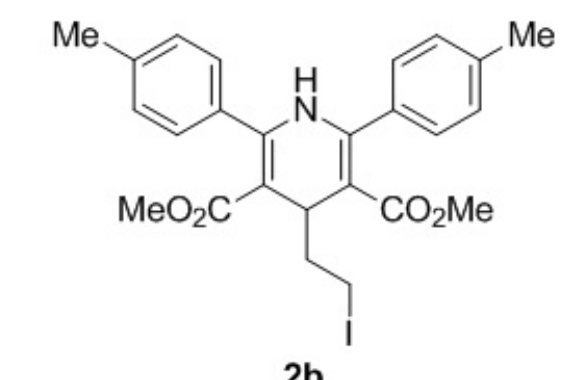

$2 b$

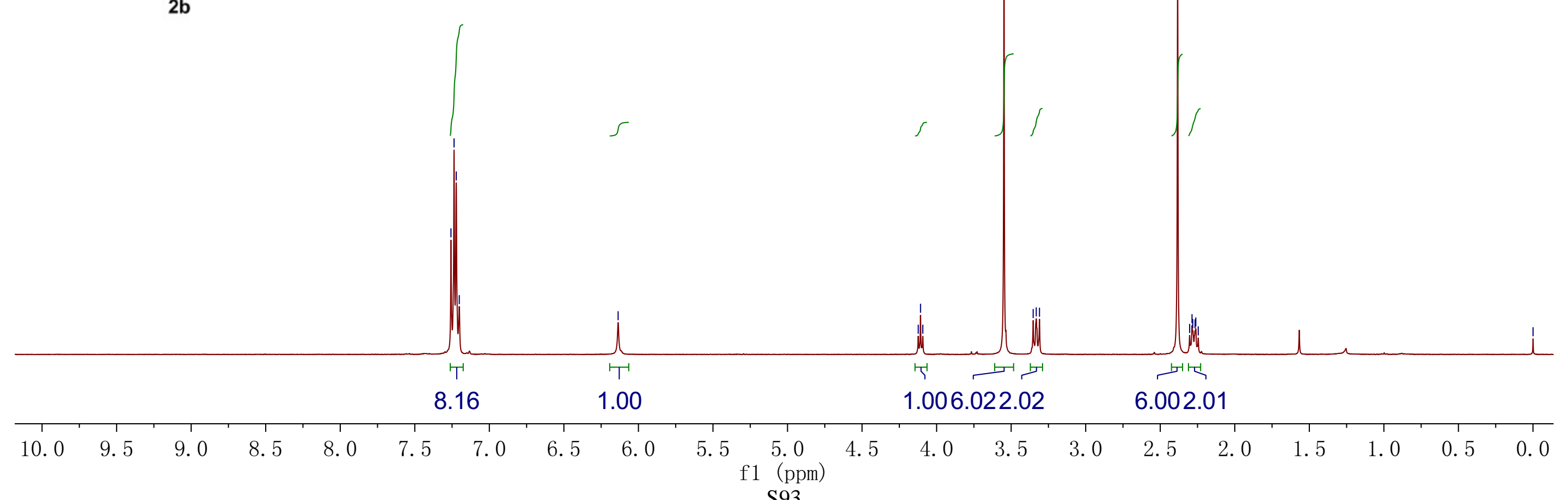




\section{${ }^{13} \mathrm{C}$ NMR (100 MHz, $\mathrm{CDCl}_{3}$ )}

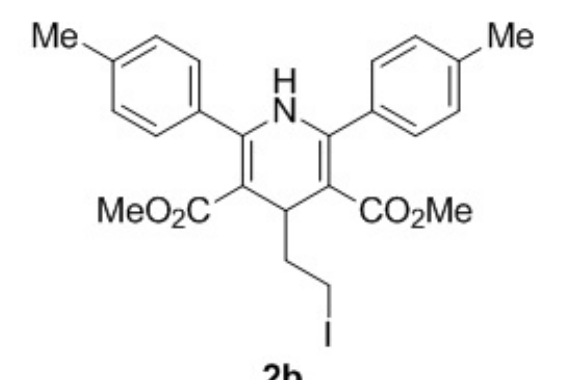

2b

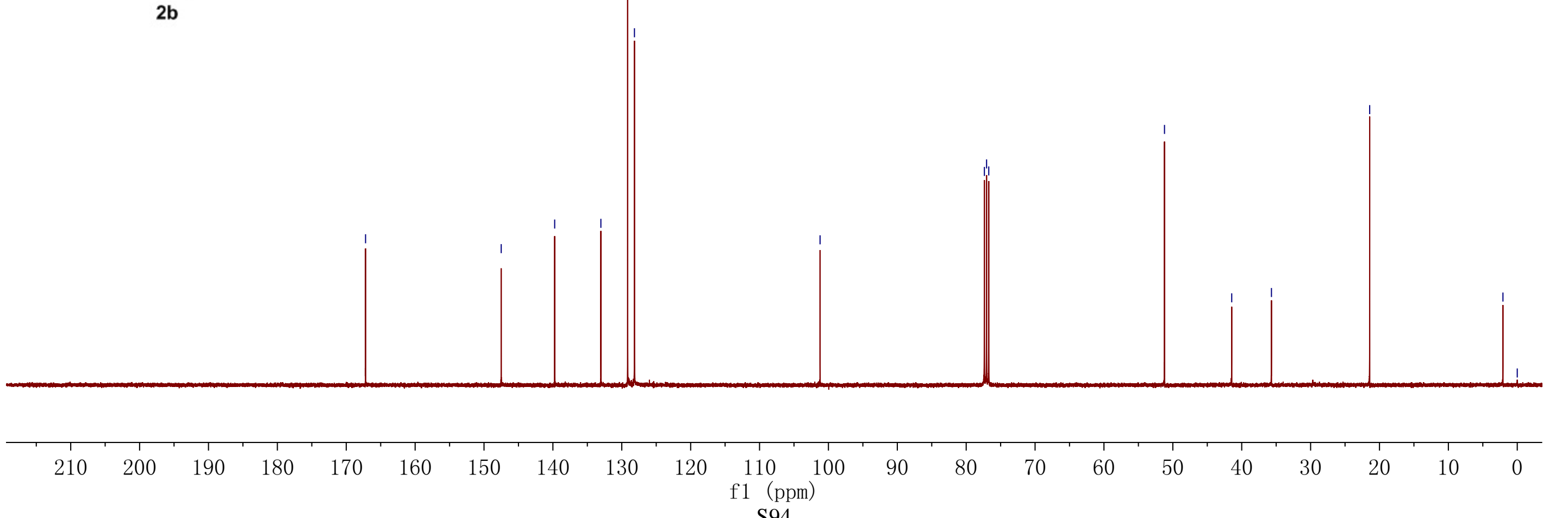


${ }^{1} \mathrm{H}$ NMR (400 MHz, $\mathrm{CDCl}_{3}$ )

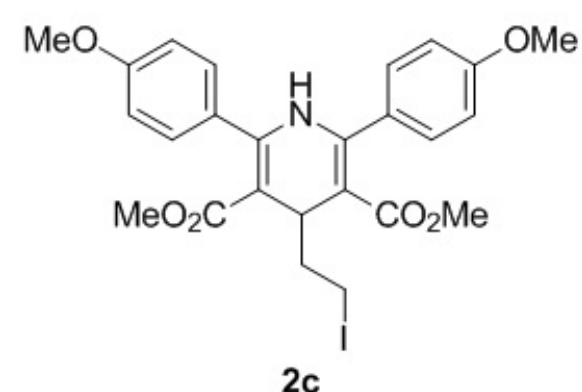

2c

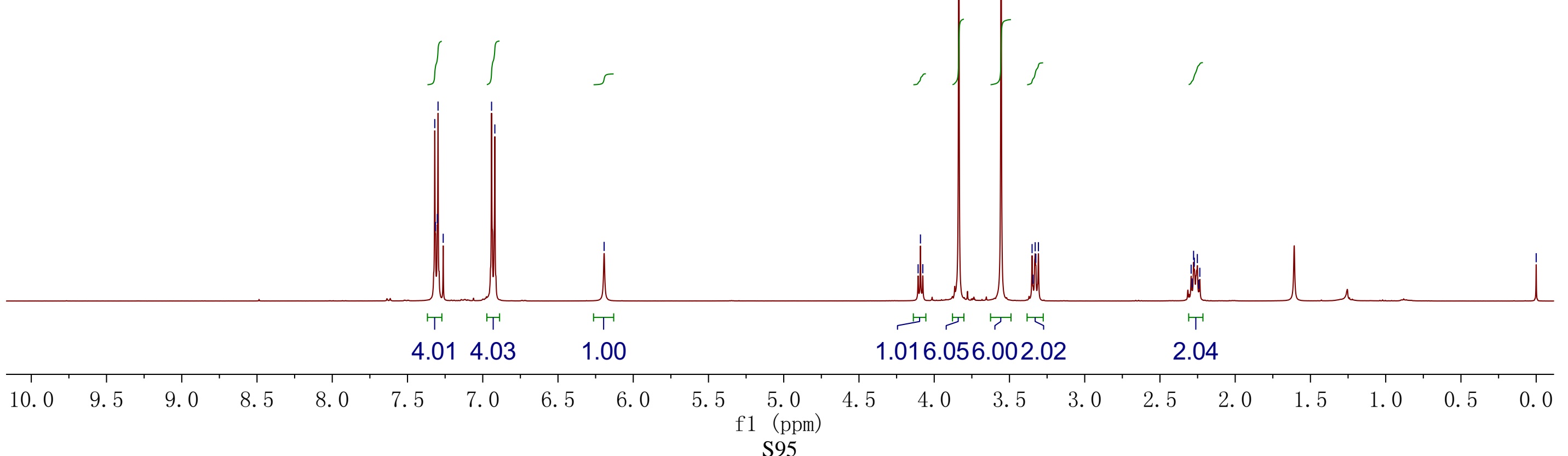




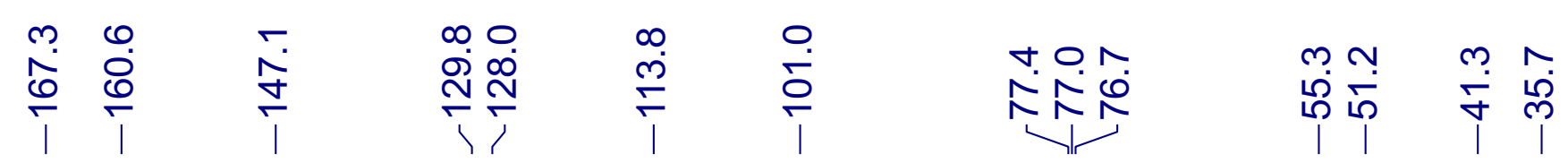

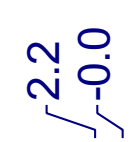

\section{${ }^{13} \mathrm{C} \mathrm{NMR}\left(100 \mathrm{MHz}, \mathrm{CDCl}_{3}\right)$}
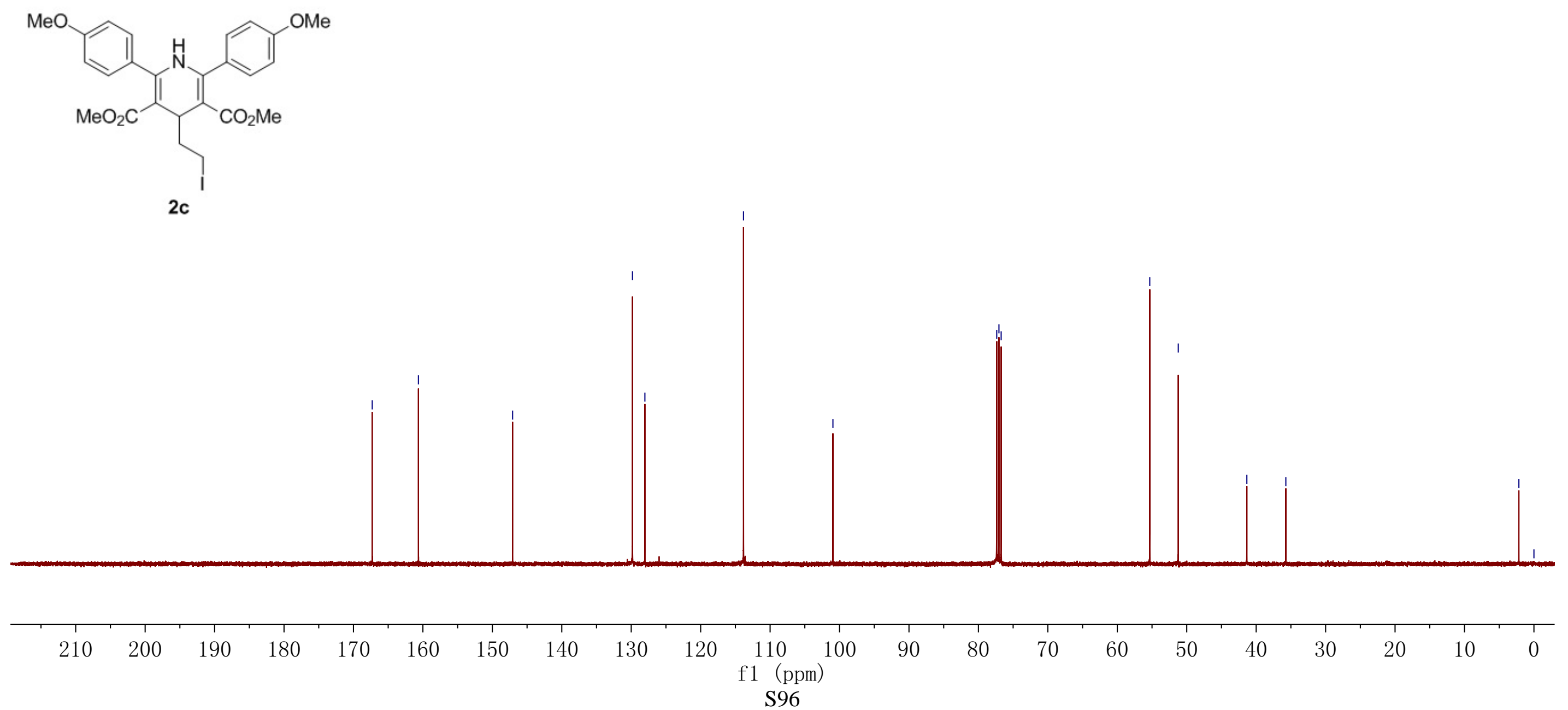
${ }^{1} \mathrm{H}$ NMR (400 MHz, $\left.\mathrm{CDCl}_{3}\right)$
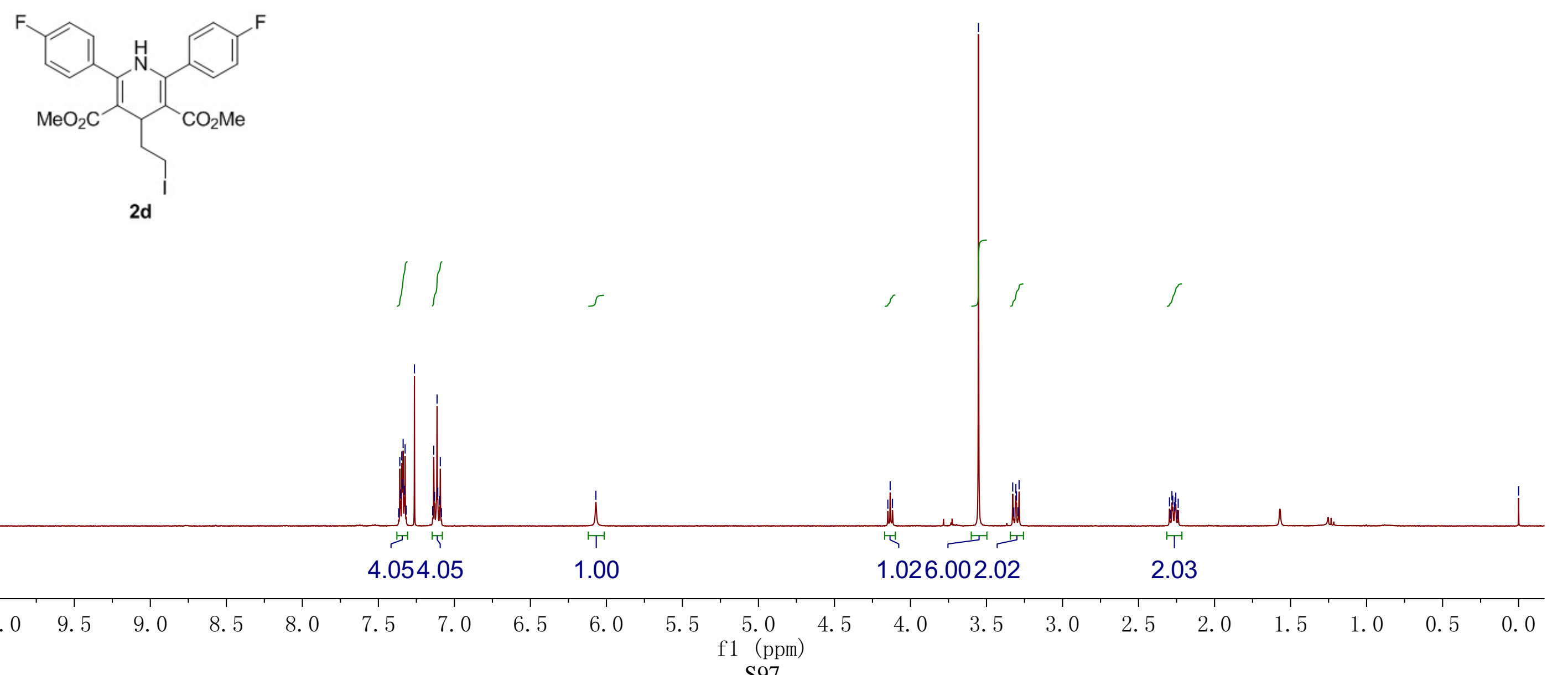
${ }^{13} \mathrm{C}$ NMR (100 MHz, $\left.\mathrm{CDCl}_{3}\right)$

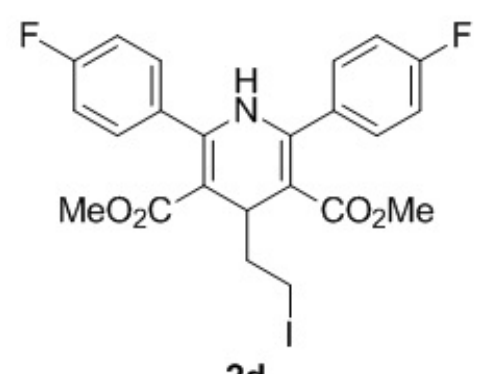

2d

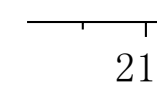

$210 \quad 200$

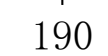


${ }^{19} \mathrm{~F}$ NMR (376 MHz, $\mathrm{CDCl}_{3}$ )
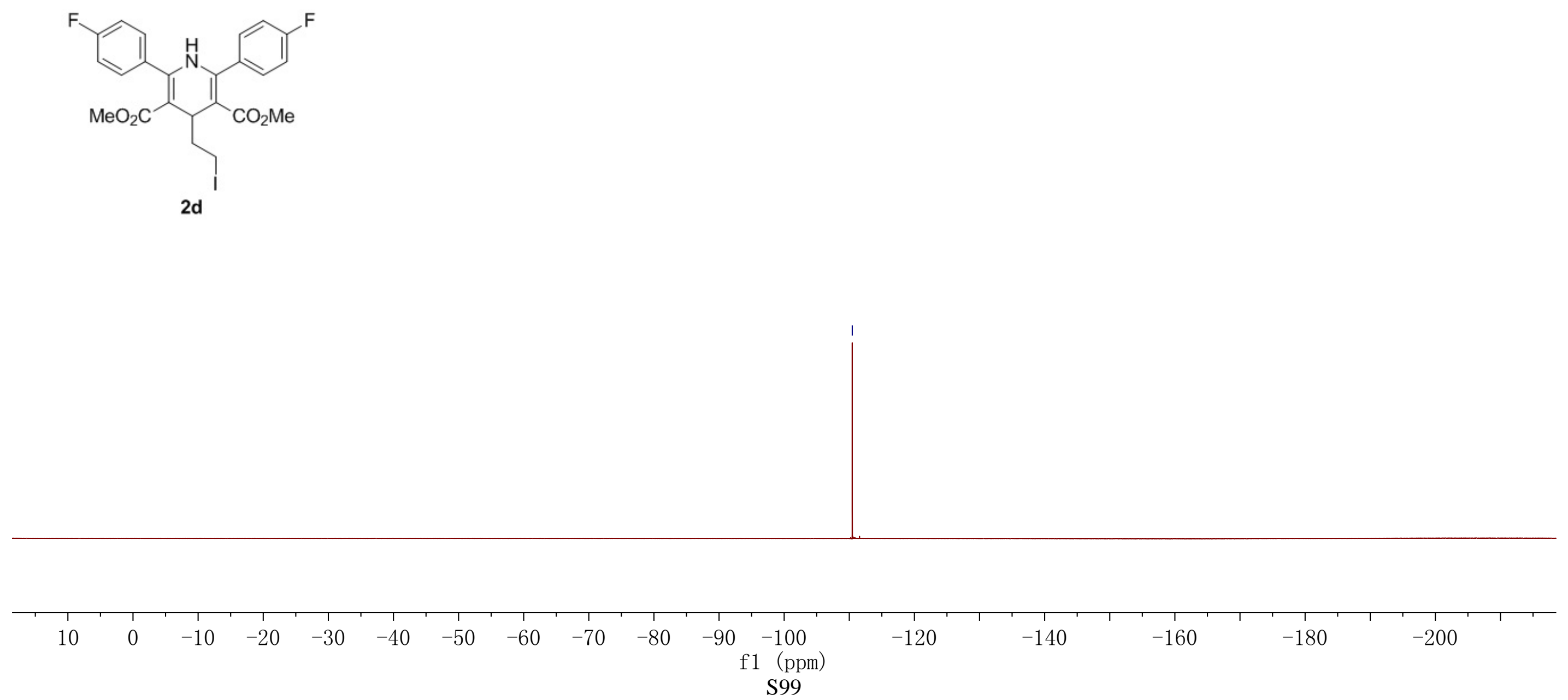
${ }^{1} \mathrm{H}$ NMR (400 MHz, $\left.\mathrm{CDCl}_{3}\right)$
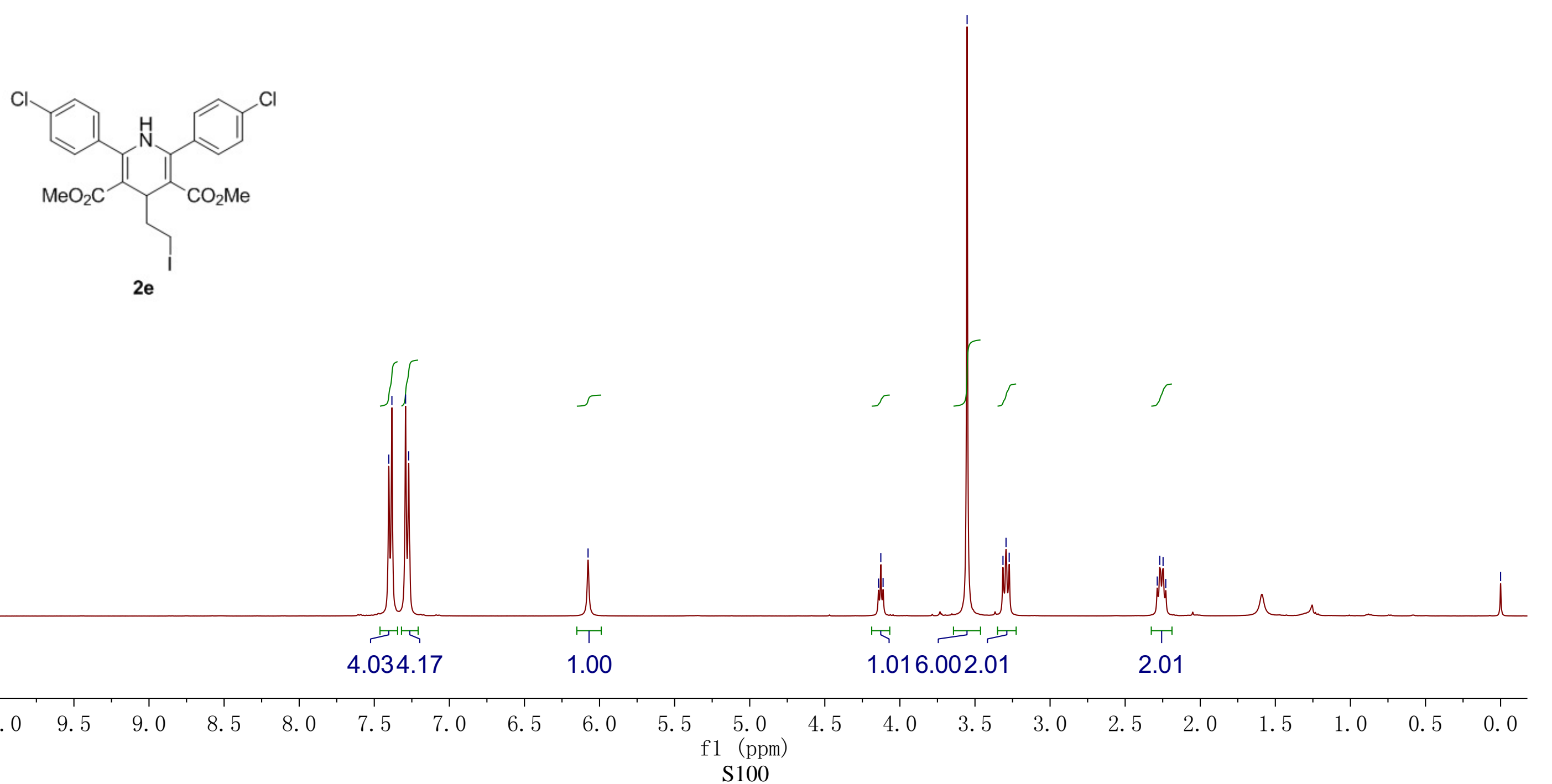
${ }^{13} \mathrm{C} \mathrm{NMR}\left(100 \mathrm{MHz}, \mathrm{CDCl}_{3}\right)$
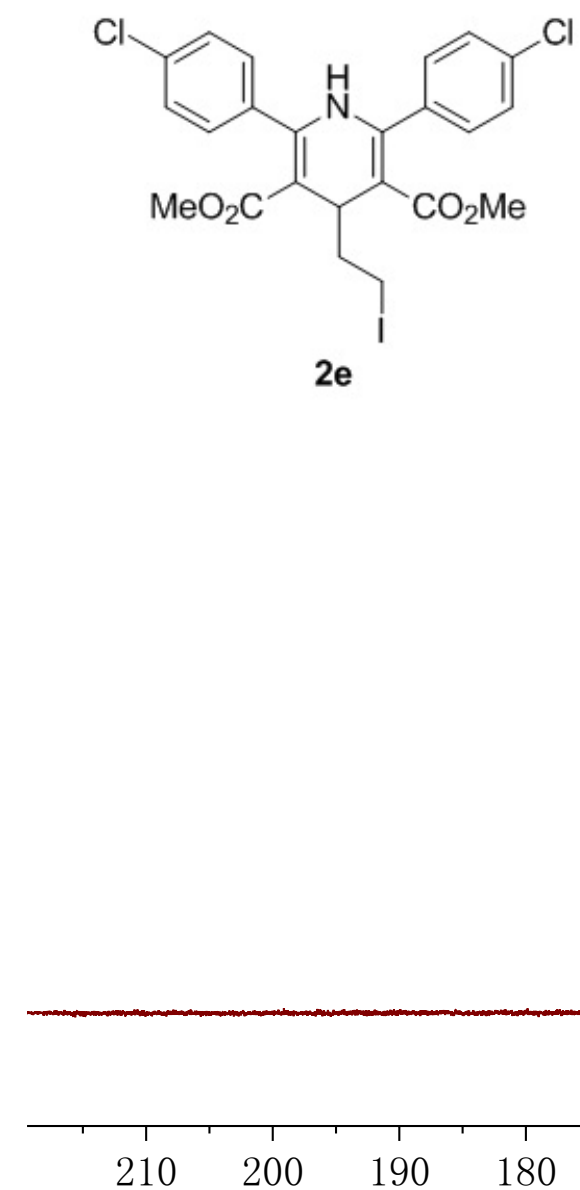

$210 \quad 200$ 
${ }^{1} \mathrm{H} \mathrm{NMR}\left(400 \mathrm{MHz}, \mathrm{CDCl}_{3}\right)$
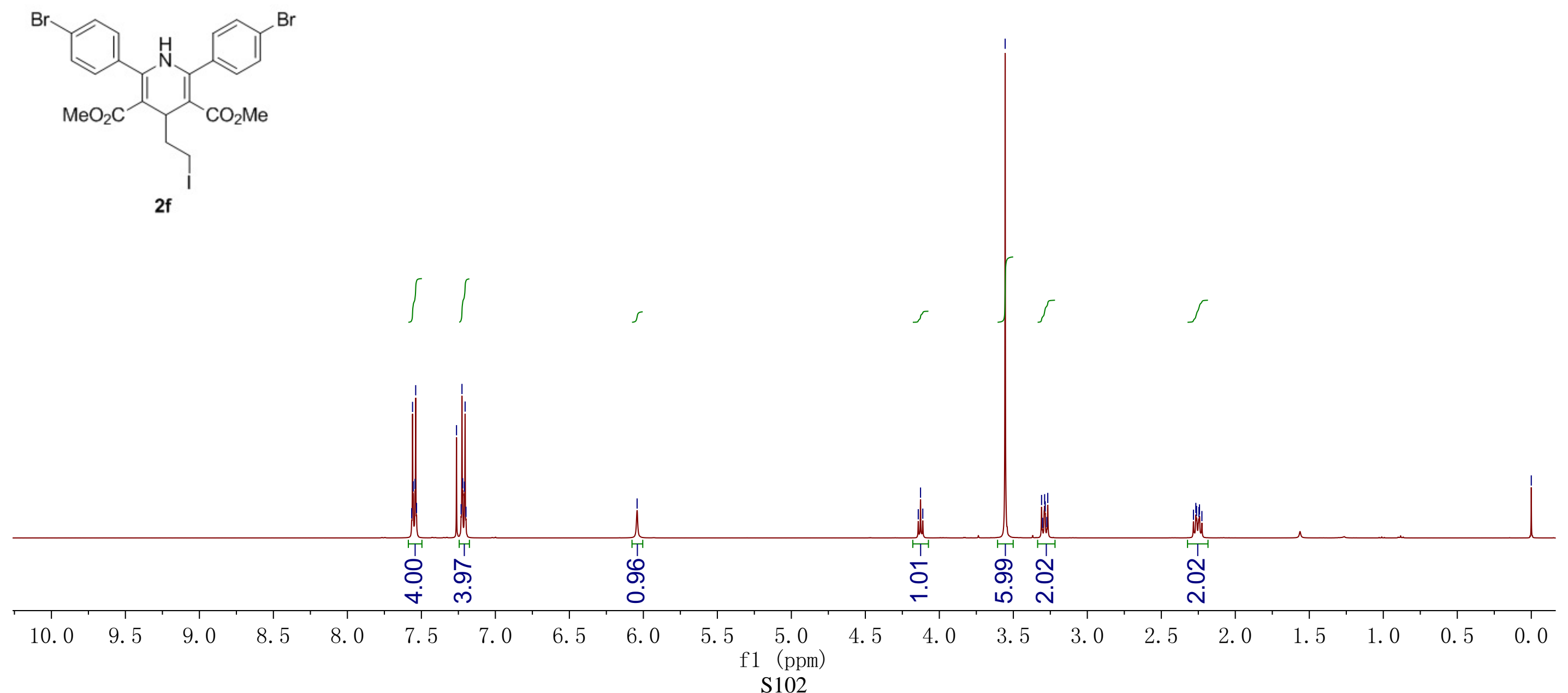


\begin{tabular}{|c|c|c|c|c|c|c|}
\hline$\frac{1}{0}$ & 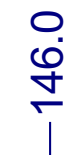 & 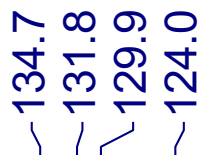 & $\stackrel{m}{0}$ & 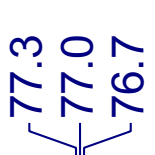 & एँ & $\frac{\dot{q}}{\dot{1}}$ \\
\hline
\end{tabular}

${ }^{13} \mathrm{C} \mathrm{NMR}\left(100 \mathrm{MHz}, \mathrm{CDCl}_{3}\right)$

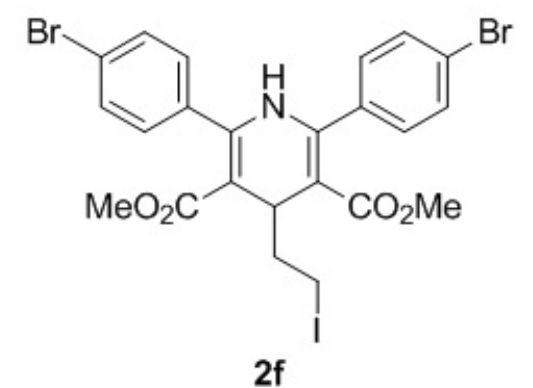

$2 f$ 
${ }^{1} \mathrm{H}$ NMR (400 MHz, $\mathrm{CDCl}_{3}$ )

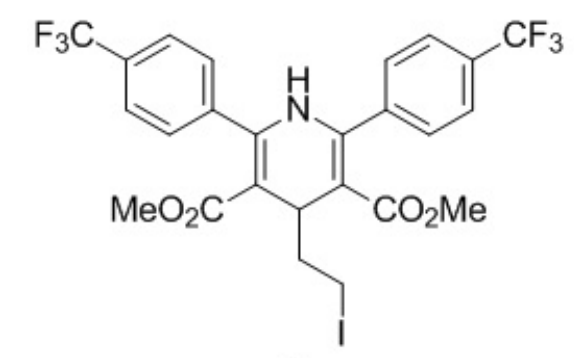

$2 \mathrm{~g}$

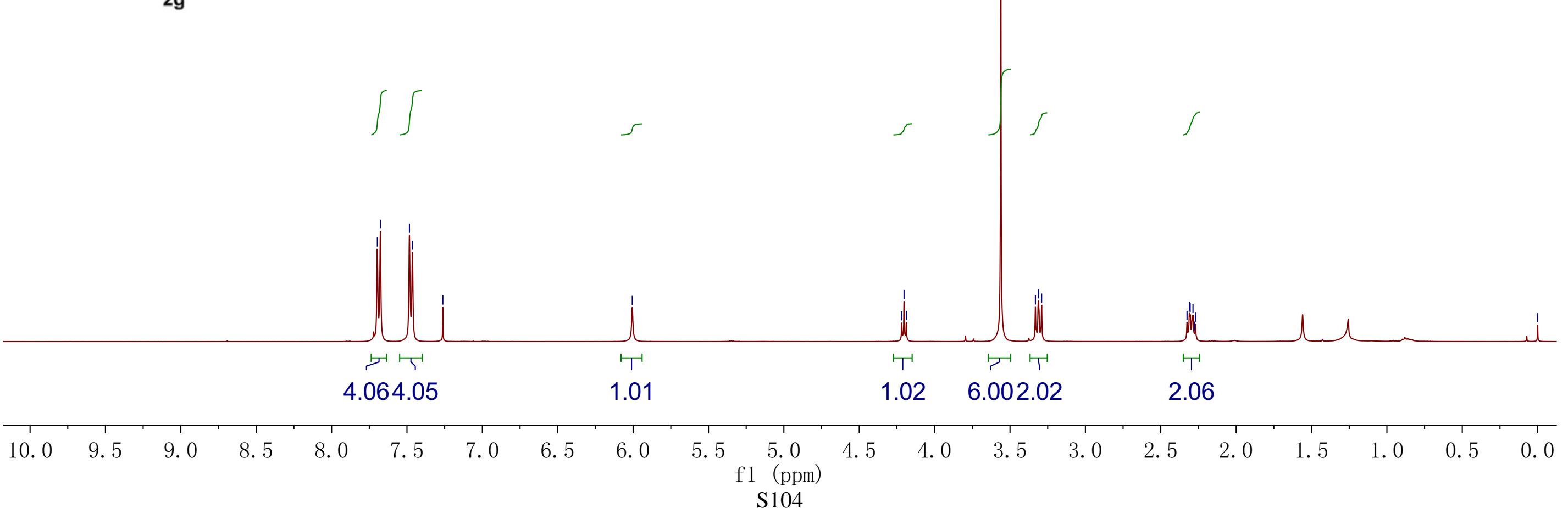




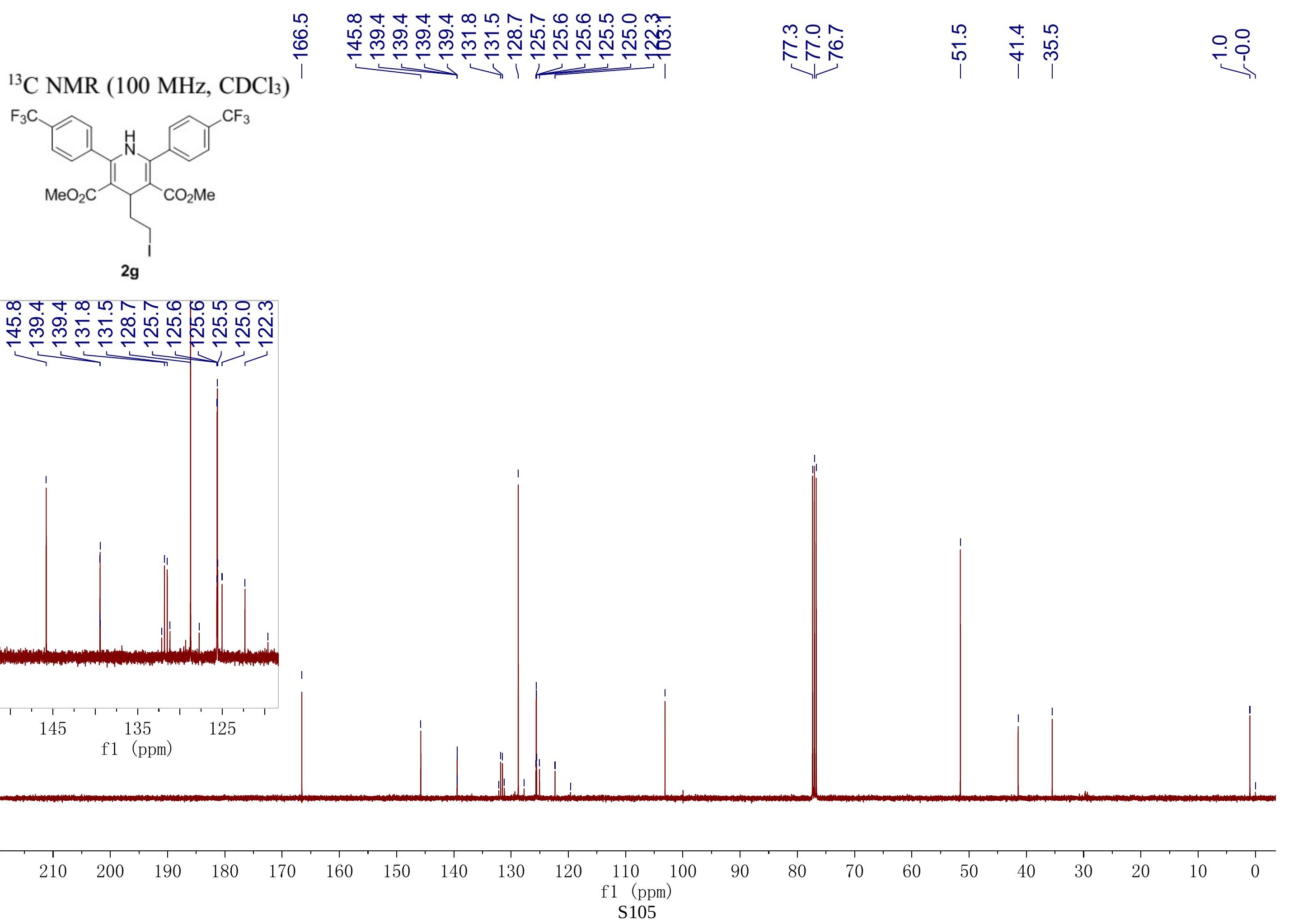


${ }^{19} \mathrm{~F}$ NMR (376 $\mathrm{MHz}, \mathrm{CDCl}_{3}$ )

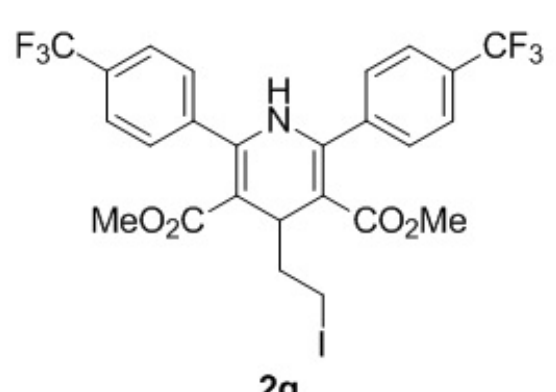

$2 g$

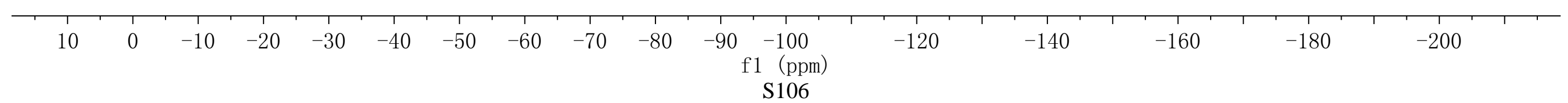


${ }^{1} \mathrm{H}$ NMR (400 MHz, $\left.\mathrm{CDCl}_{3}\right)$

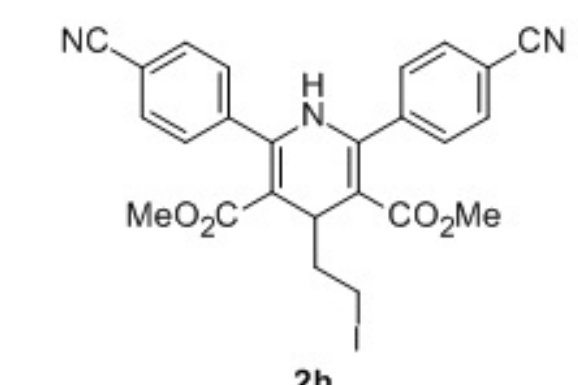

2h

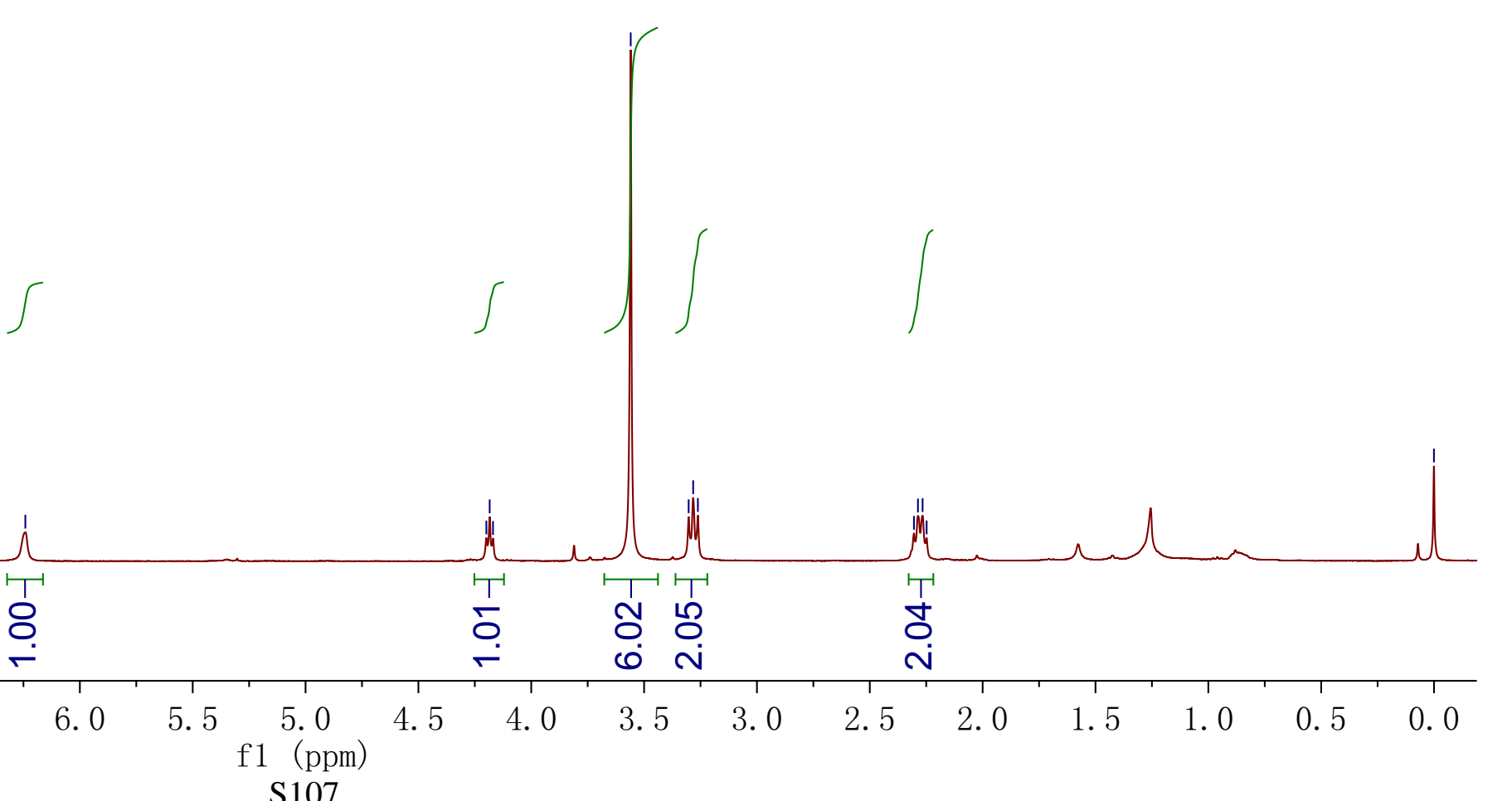


${ }^{13} \mathrm{C} \mathrm{NMR}\left(100 \mathrm{MHz}, \mathrm{CDCl}_{3}\right)$

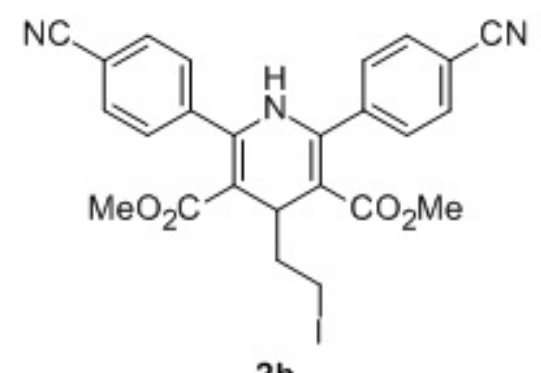

$2 \mathrm{~h}$

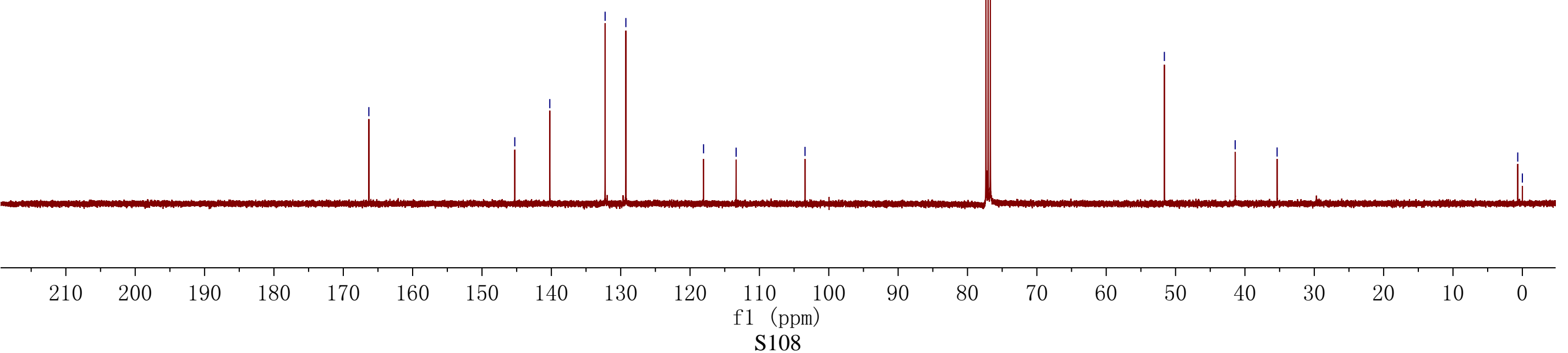


${ }^{1} \mathrm{H} \mathrm{NMR}\left(400 \mathrm{MHz}, \mathrm{CDCl}_{3}\right)$

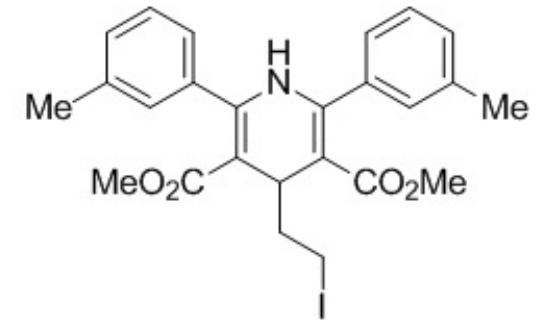

2i

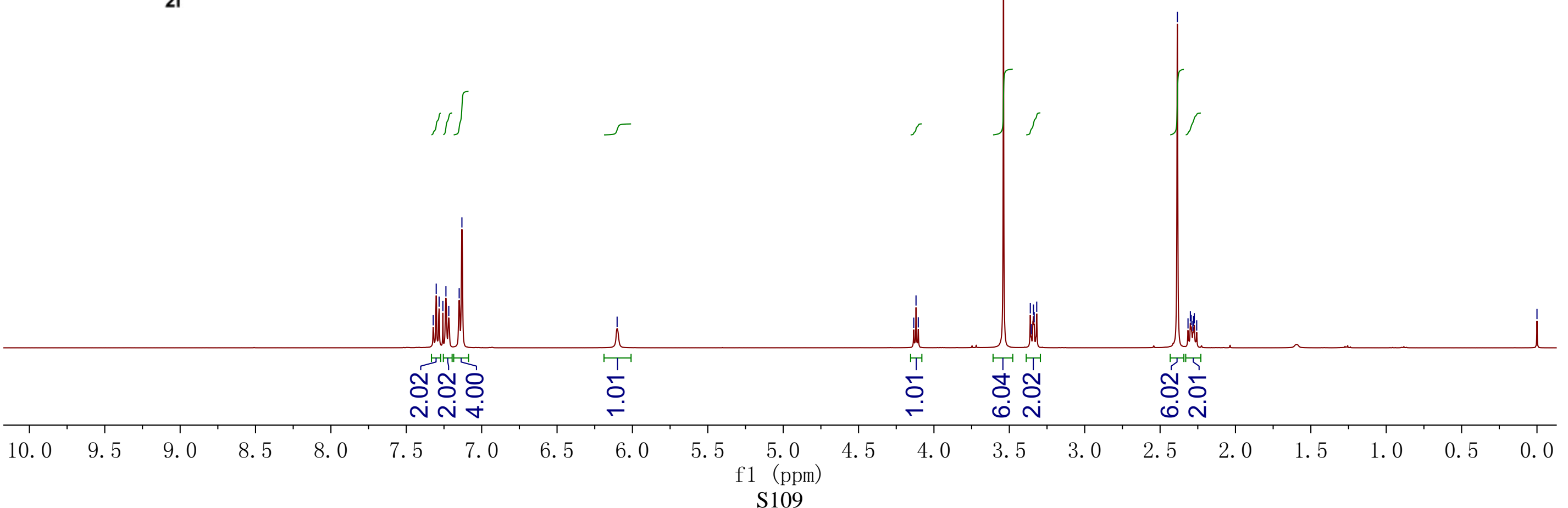


${ }^{13} \mathrm{C} \mathrm{NMR}\left(100 \mathrm{MHz}, \mathrm{CDCl}_{3}\right)$

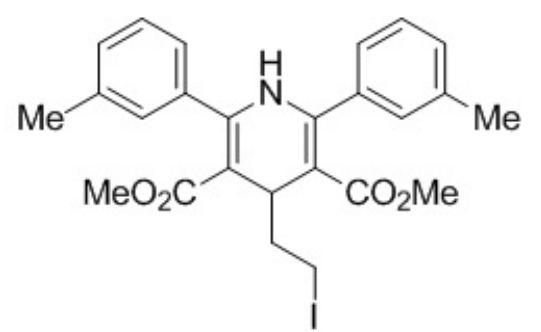

2i

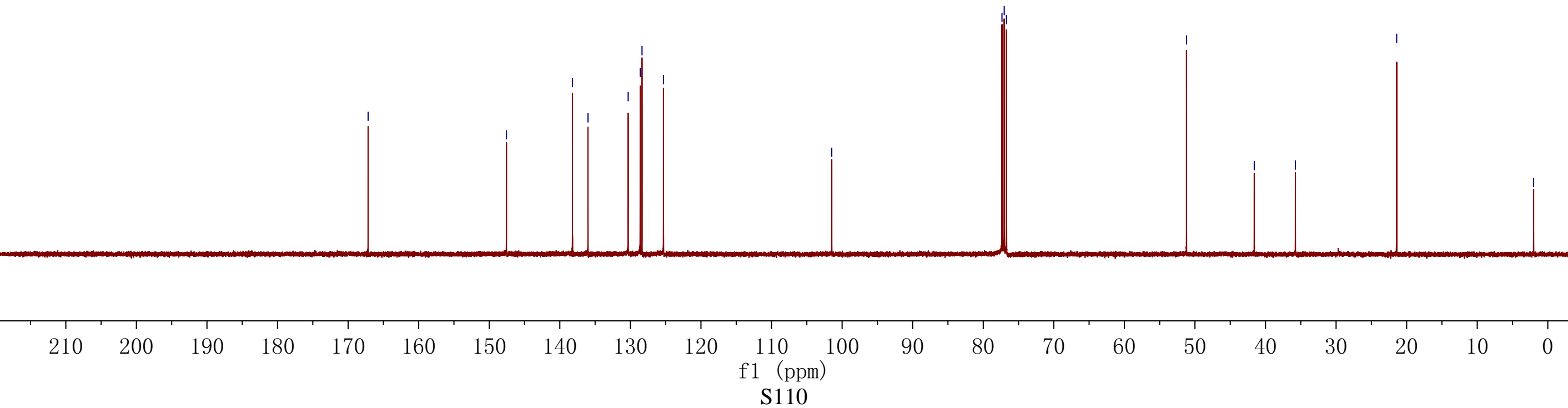


${ }^{1} \mathrm{H}$ NMR (400 MHz, $\mathrm{CDCl}_{3}$ )
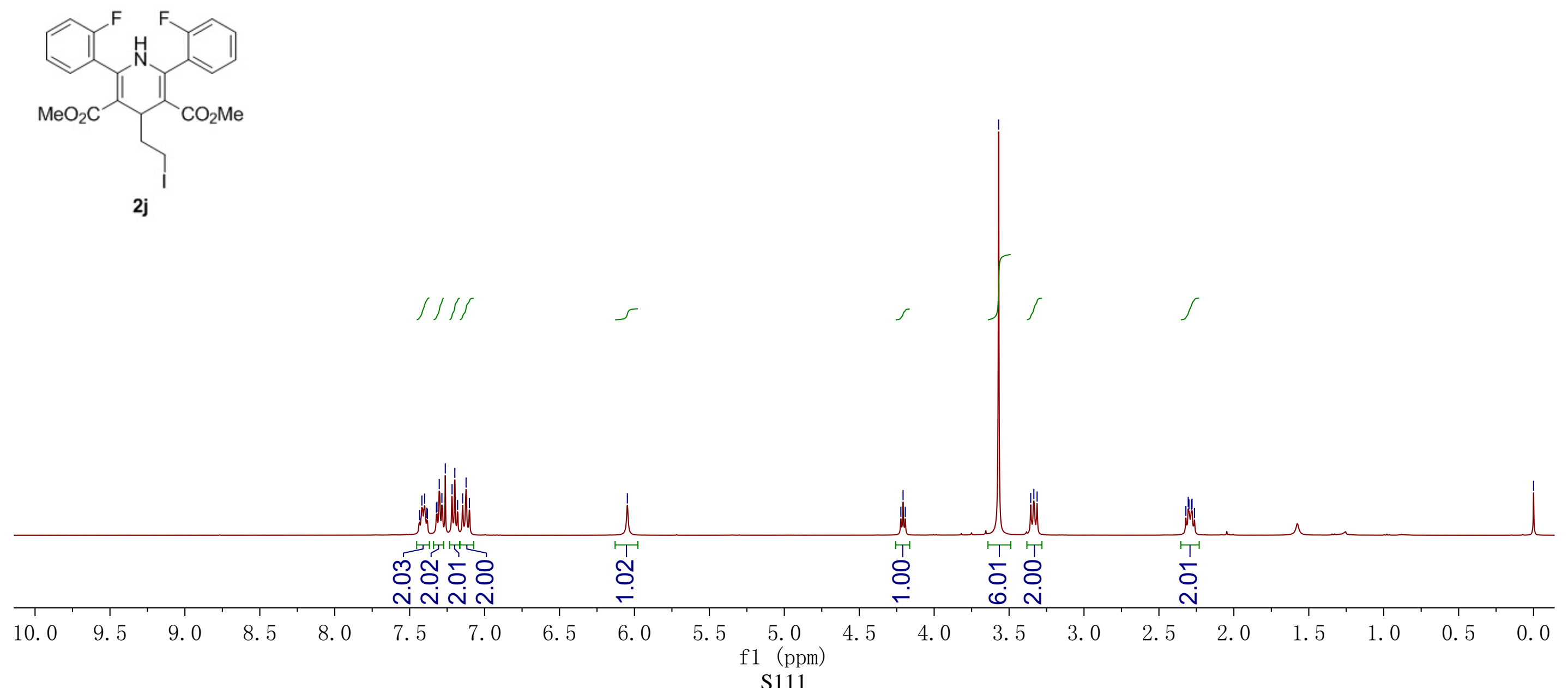
${ }^{13} \mathrm{C}$ NMR (100 MHz, $\left.\mathrm{CDCl}_{3}\right)$

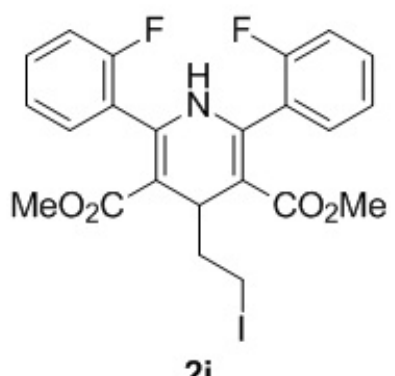

2j

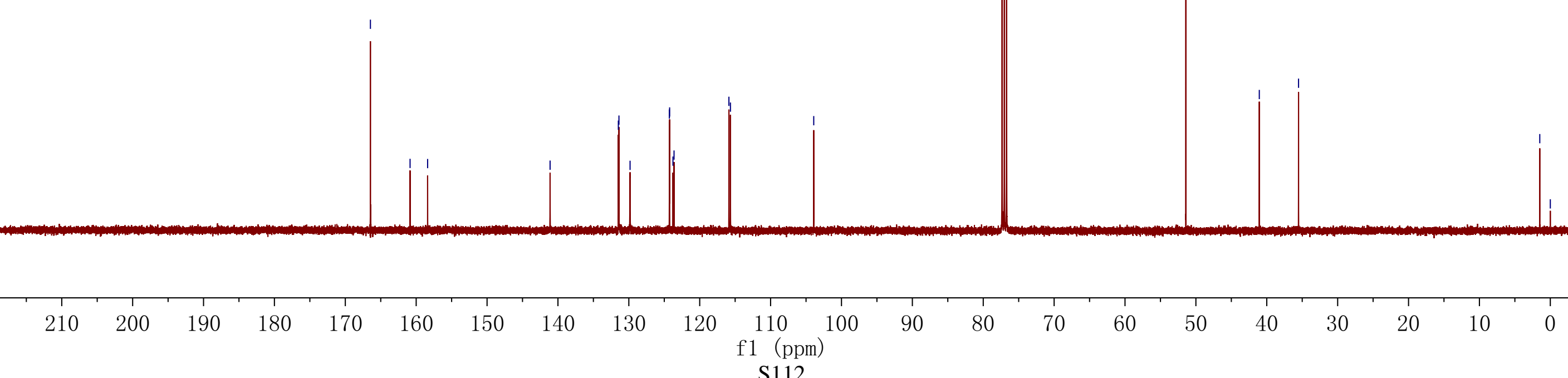


${ }^{19} \mathrm{~F}$ NMR (376 $\mathrm{MHz}, \mathrm{CDCl}_{3}$ )

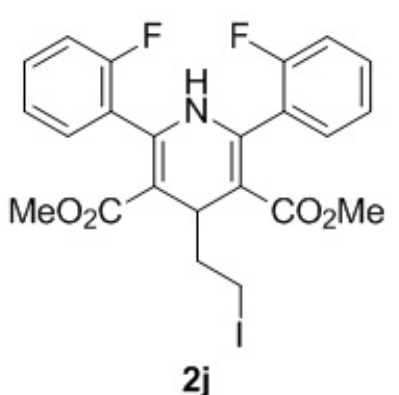

2j

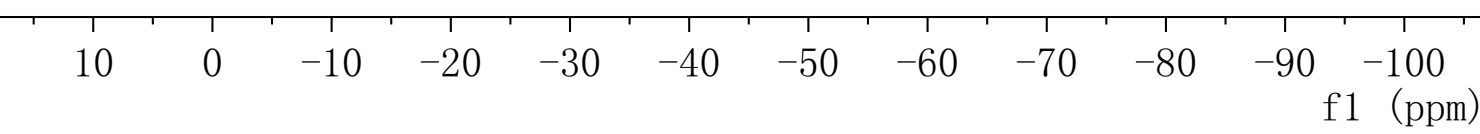

S113 
${ }^{1} \mathrm{H}$ NMR (400 MHz, $\mathrm{CDCl}_{3}$ )
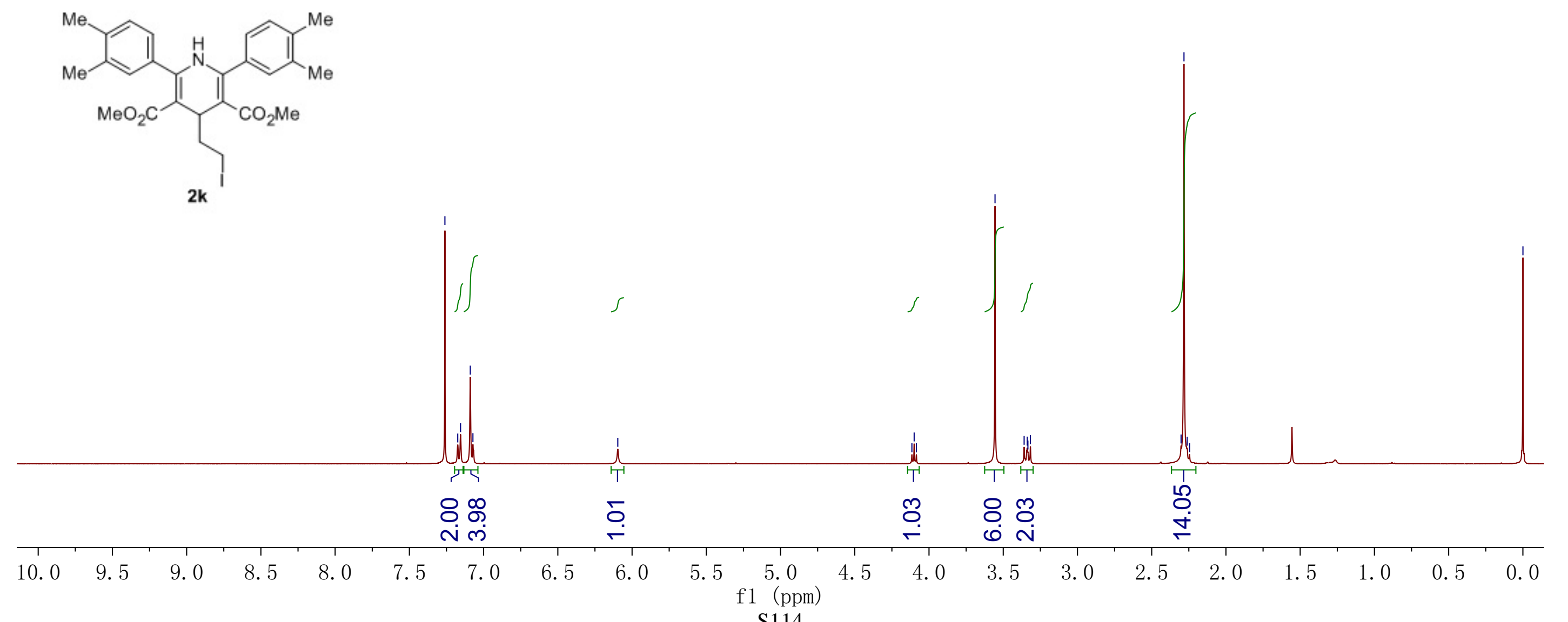
${ }^{13} \mathrm{C} \mathrm{NMR}\left(100 \mathrm{MHz}, \mathrm{CDCl}_{3}\right)$
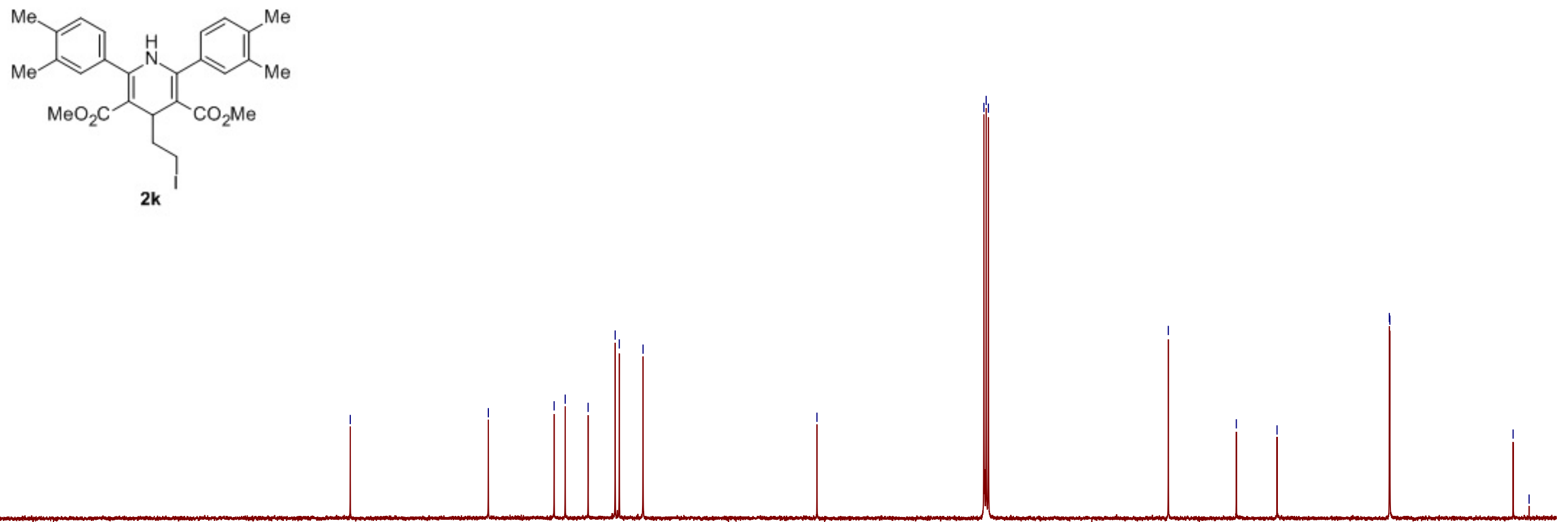
${ }^{1} \mathrm{H} \mathrm{NMR}\left(400 \mathrm{MHz}, \mathrm{CDCl}_{3}\right)$
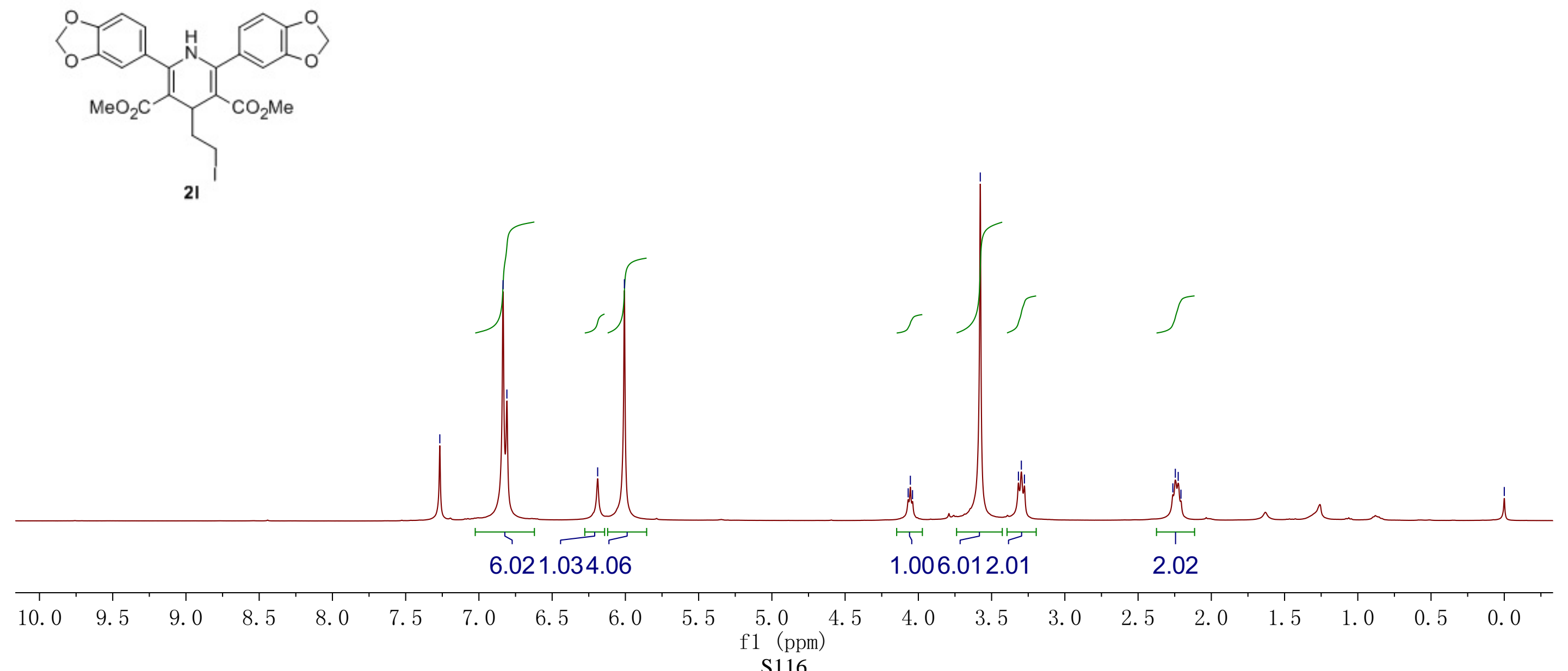


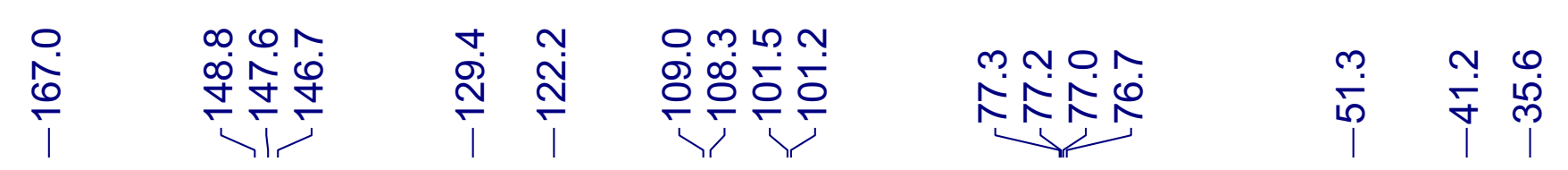

${ }^{13} \mathrm{C} \mathrm{NMR}\left(100 \mathrm{MHz}, \mathrm{CDCl}_{3}\right)$
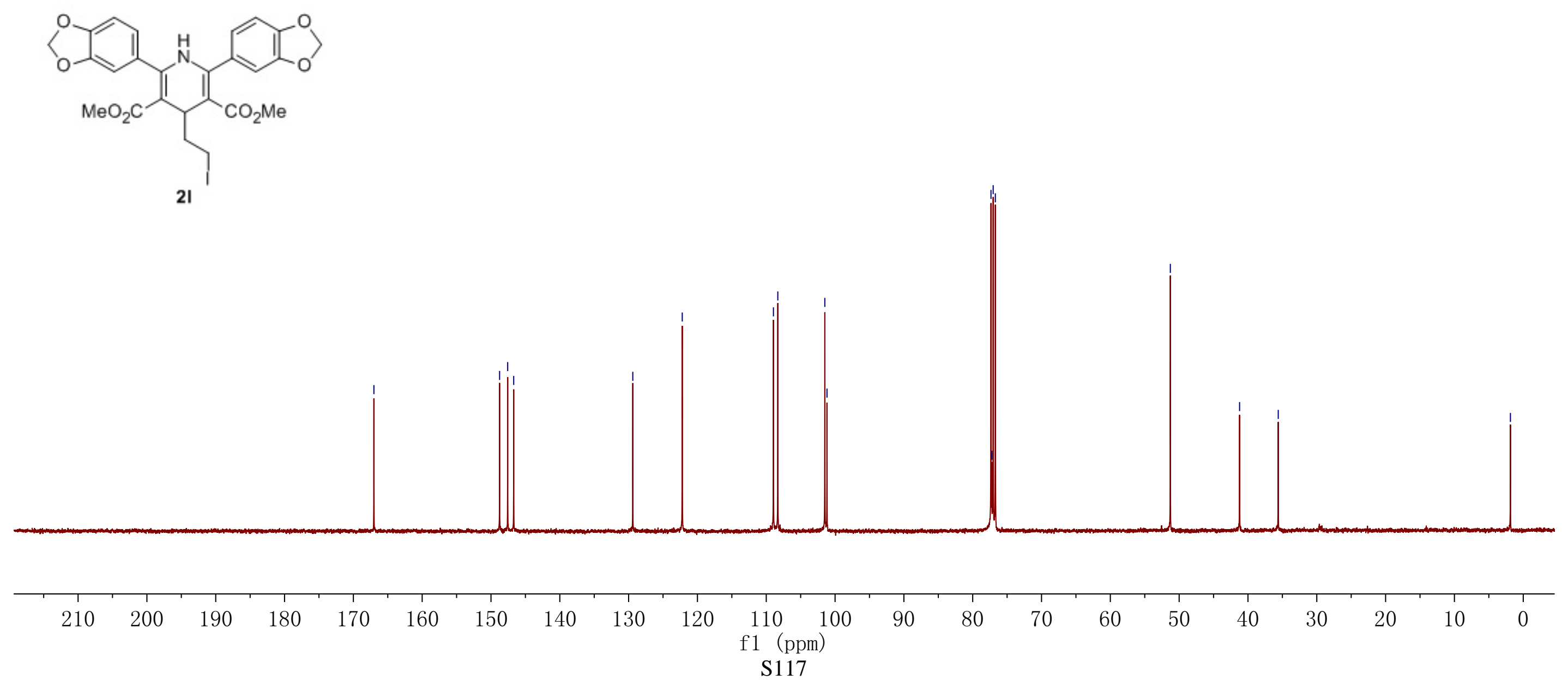
${ }^{1} \mathrm{H}$ NMR (400 MHz, $\left.\mathrm{CDCl}_{3}\right)$

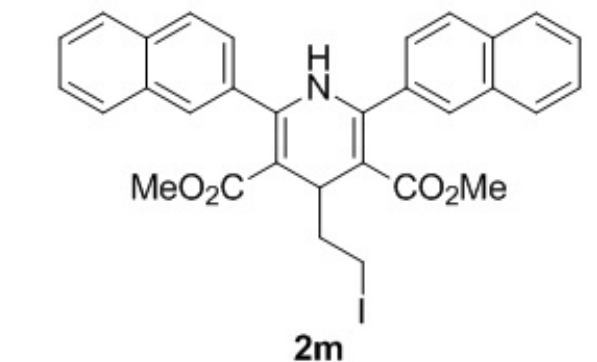

$2 \mathrm{~m}$

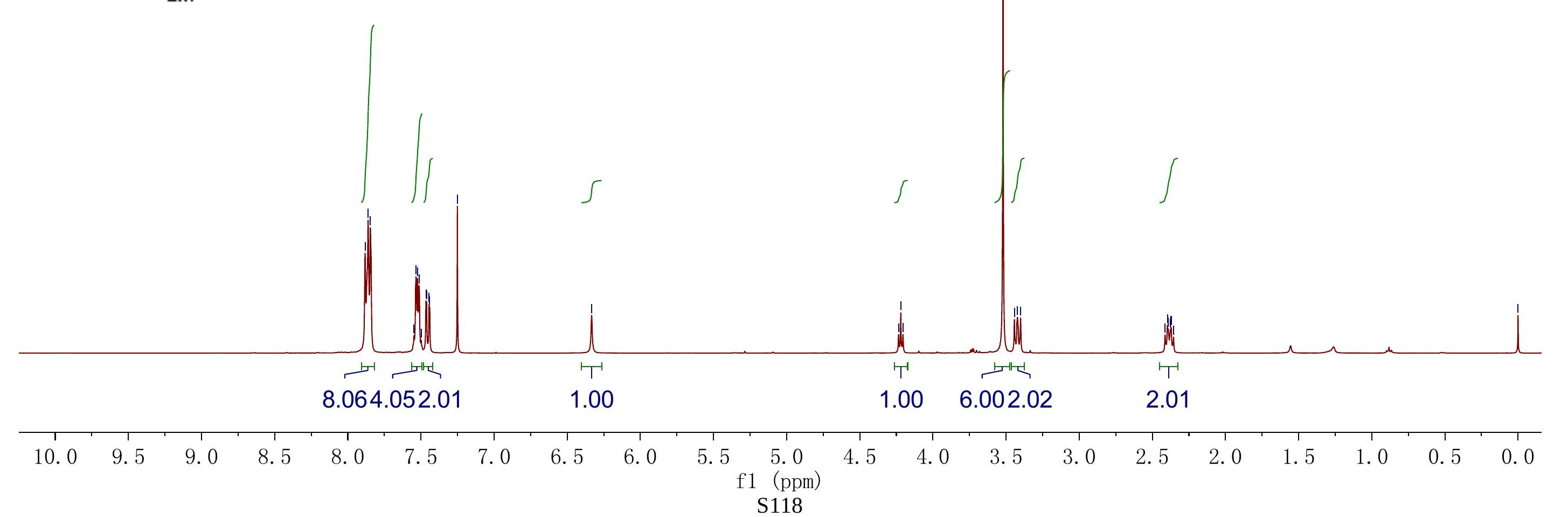


${ }^{13} \mathrm{C} \mathrm{NMR}\left(100 \mathrm{MHz}, \mathrm{CDCl}_{3}\right)$

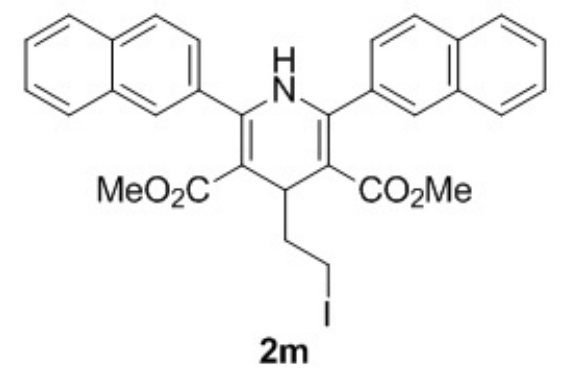

$2 \mathrm{~m}$

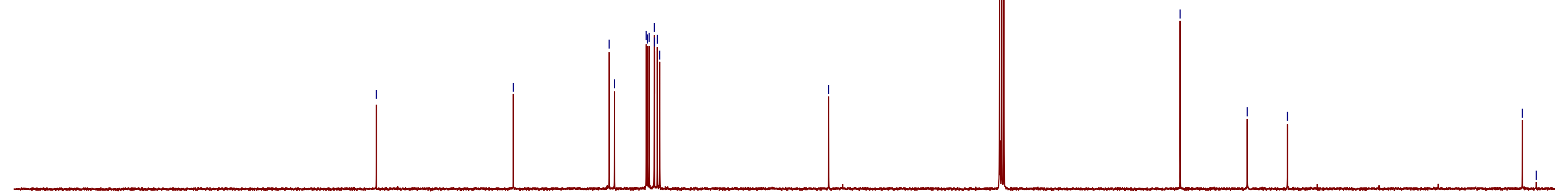


${ }^{1} \mathrm{H}$ NMR (400 MHz, $\mathrm{CDCl}_{3}$ )
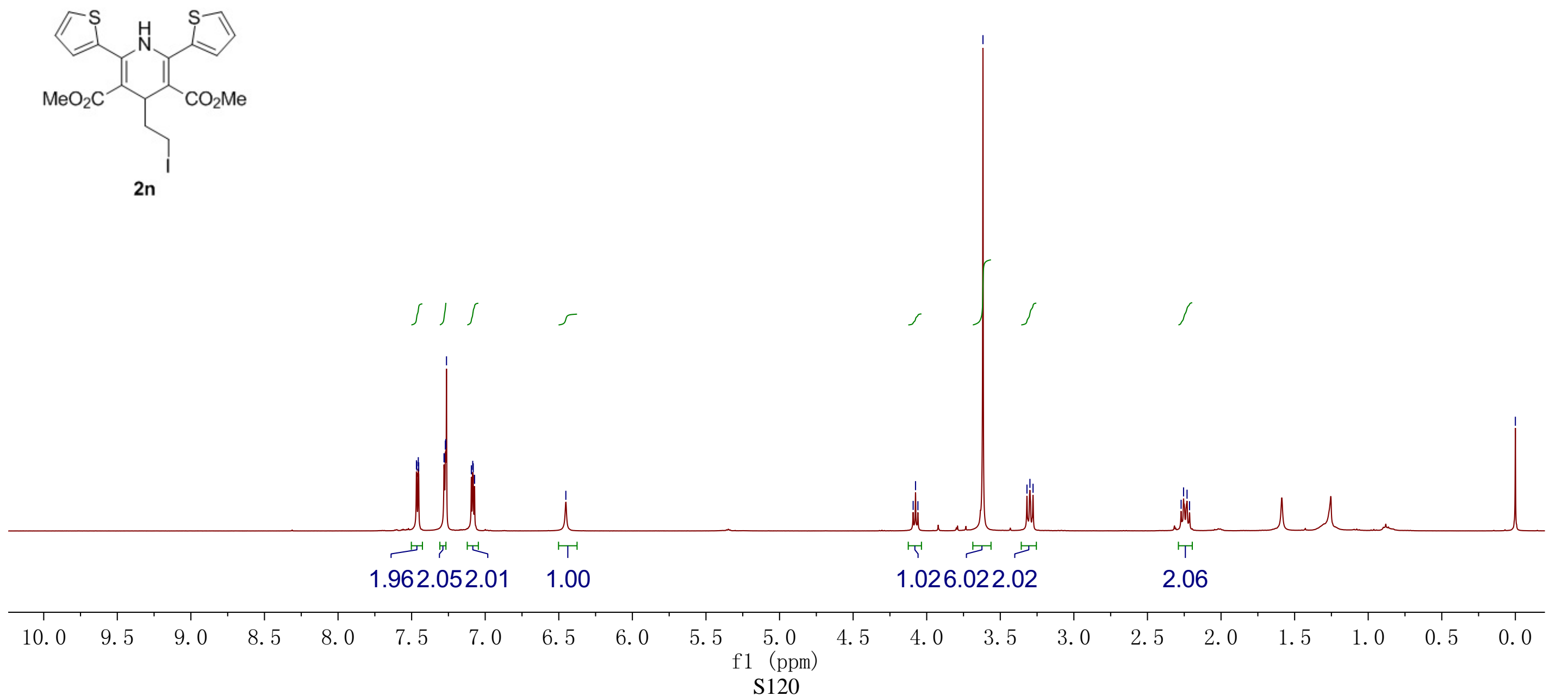
${ }^{13} \mathrm{C} \mathrm{NMR}\left(100 \mathrm{MHz}, \mathrm{CDCl}_{3}\right)$

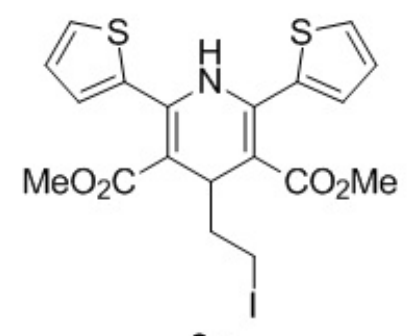

2n

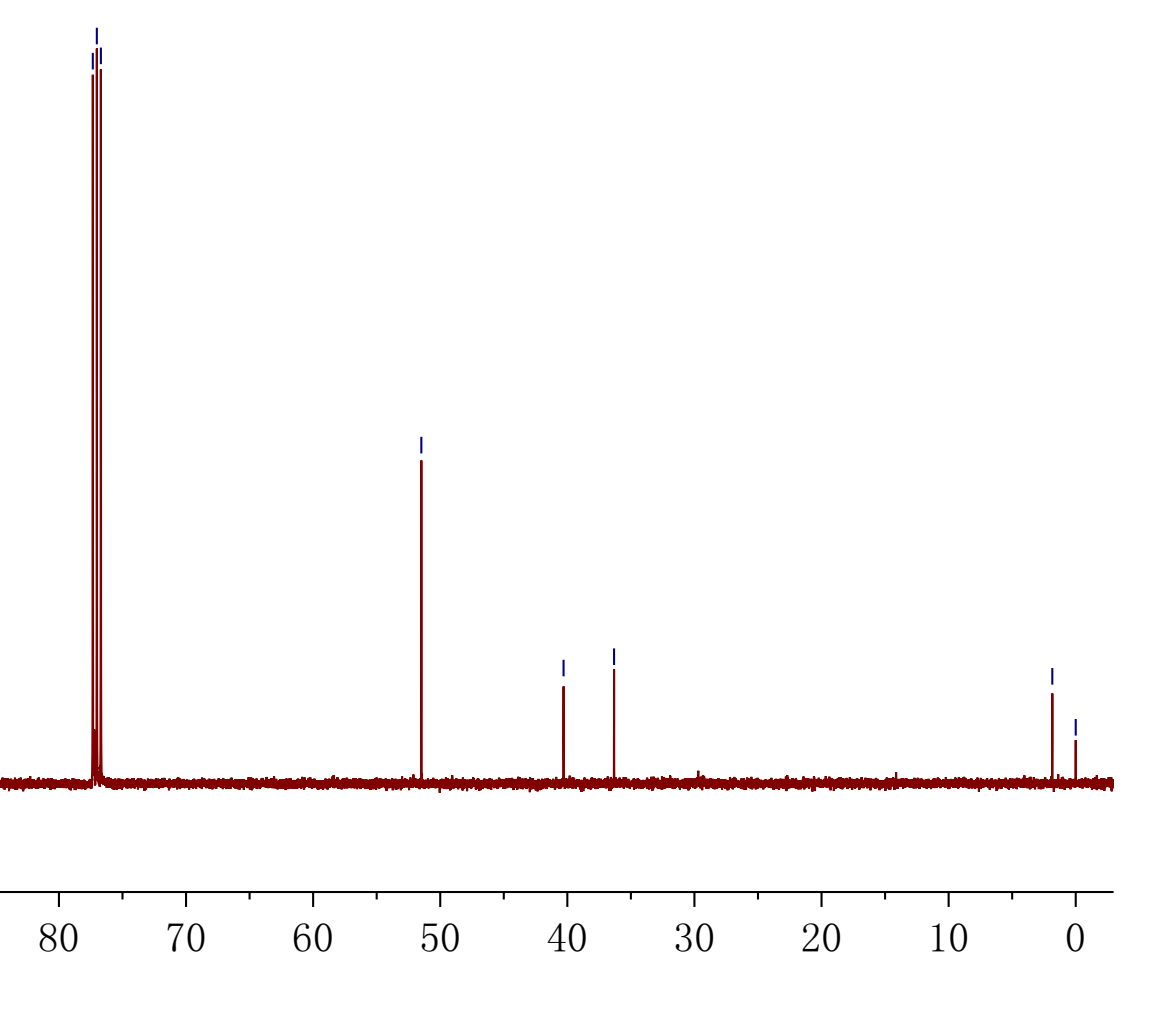


అம

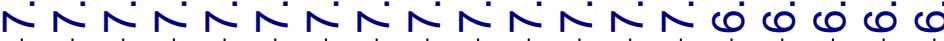
${ }^{1} \mathrm{H}$ NMR $\left(400 \mathrm{MHz}, \mathrm{CDCl}_{3}\right)$

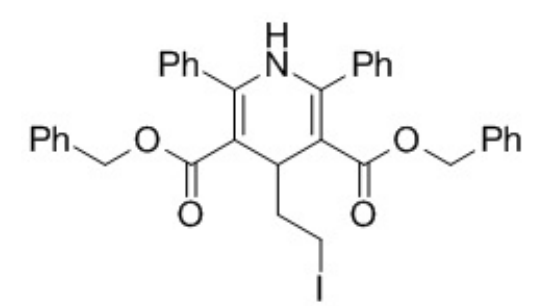

20

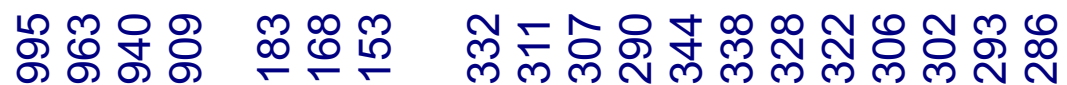

ल

min m

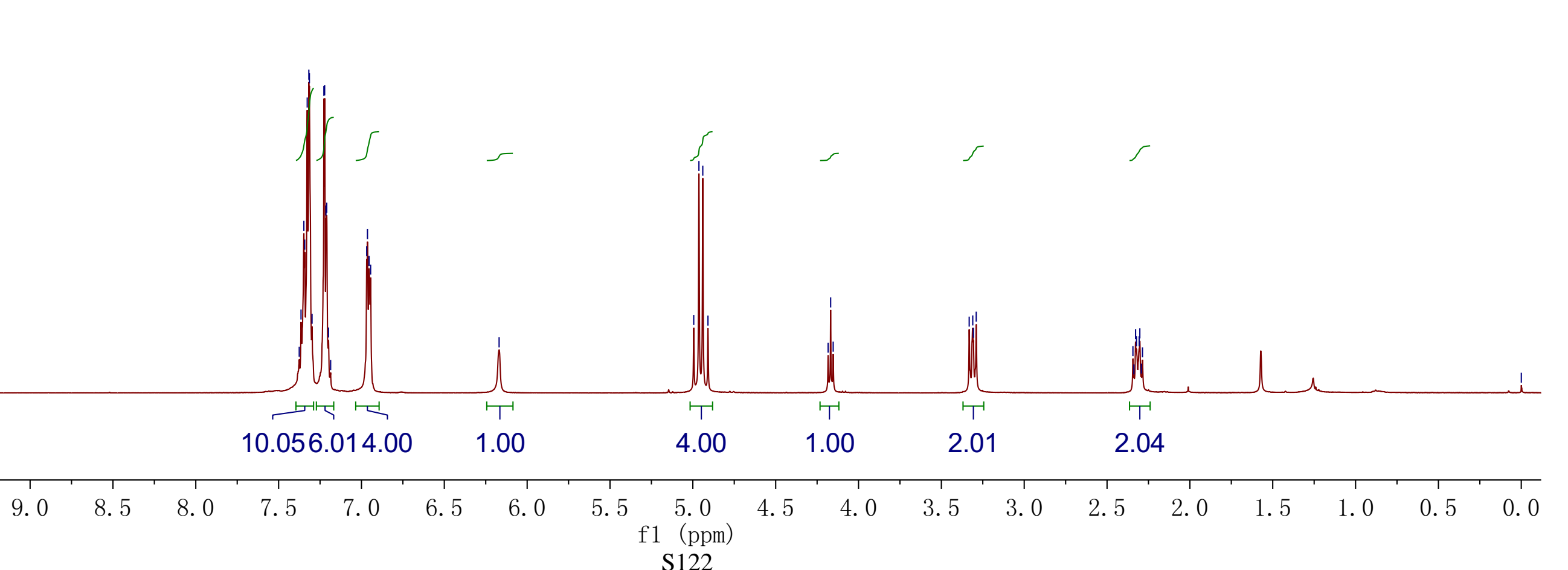




\section{${ }^{13} \mathrm{C}$ NMR (100 MHz, $\mathrm{CDCl}_{3}$ )}

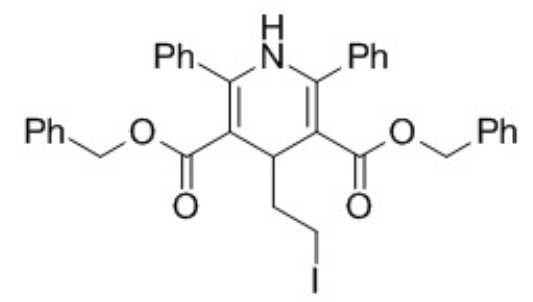

20

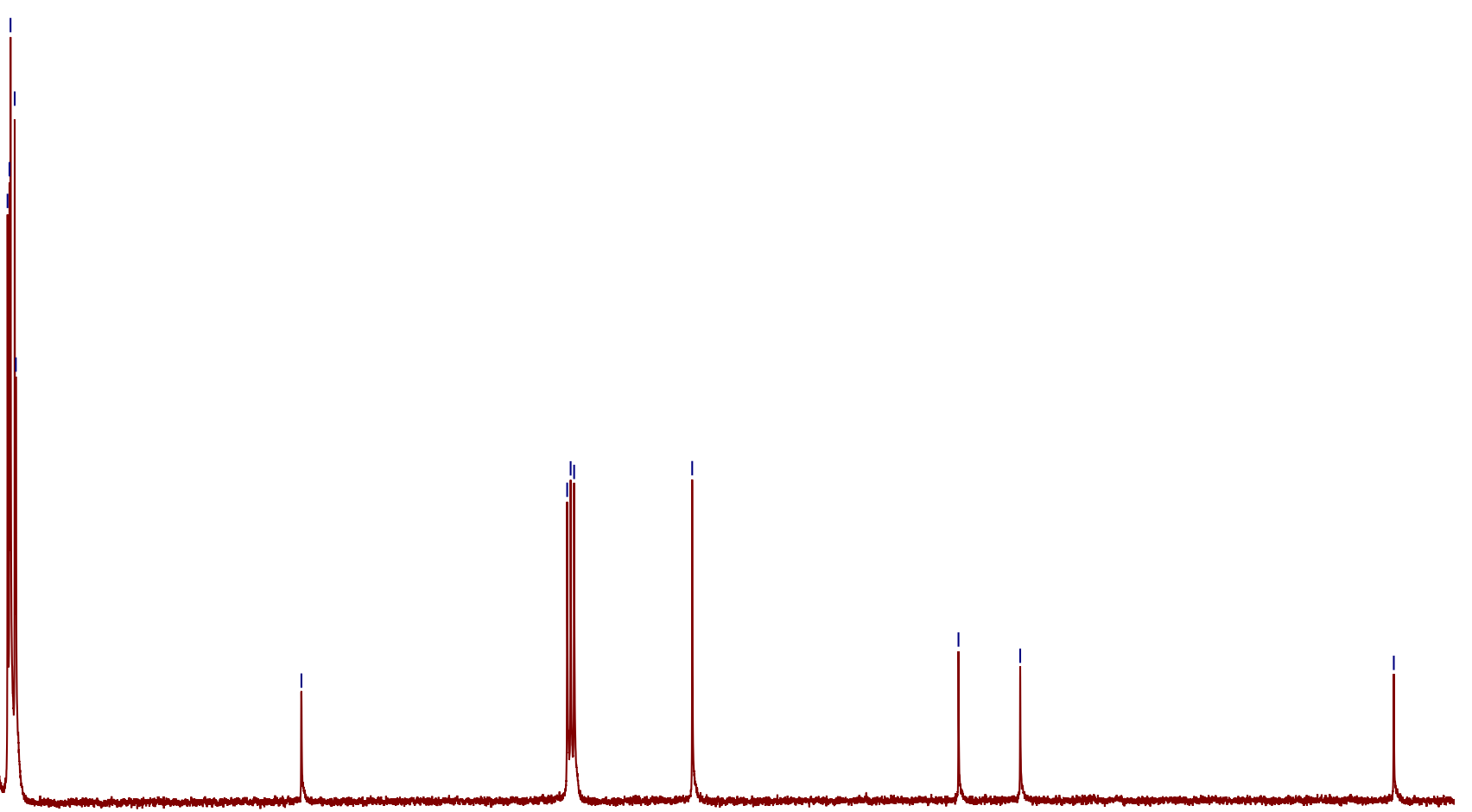

210

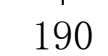

180

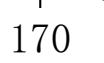

160

150

140

130

120

110

1 (ppm) 
${ }^{1} \mathrm{H}$ NMR (400 MHz, $\mathrm{CDCl}_{3}$ )

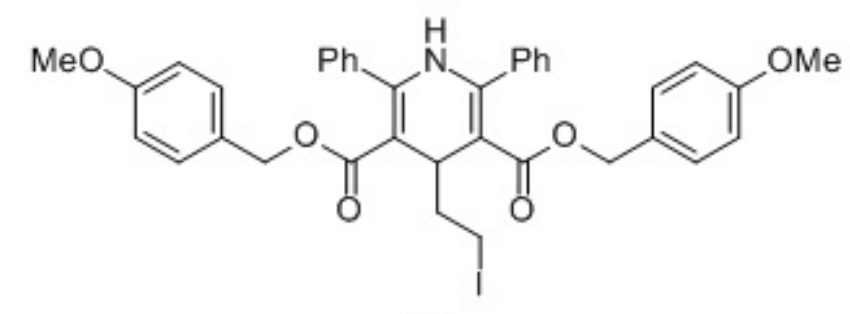

$2 p$

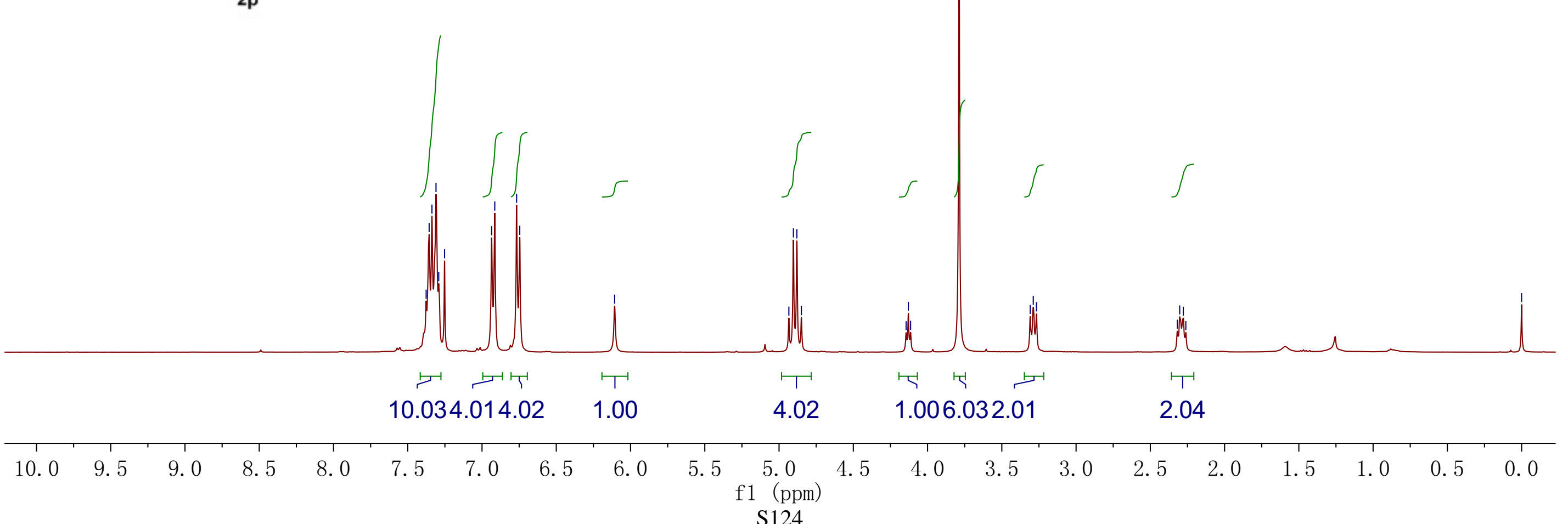




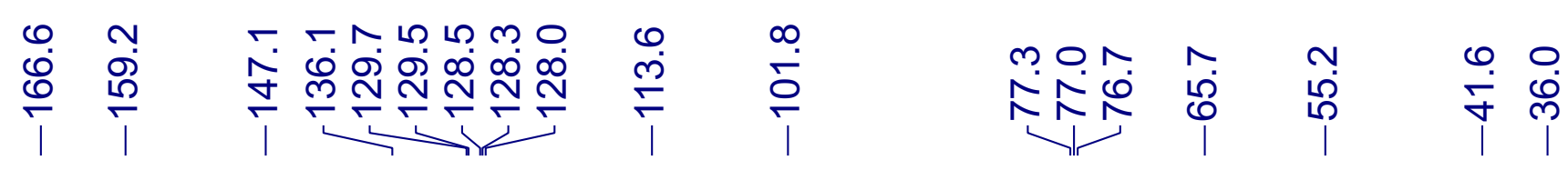

$\underbrace{\circ}$

\section{${ }^{13} \mathrm{C} \mathrm{NMR}\left(100 \mathrm{MHz}, \mathrm{CDCl}_{3}\right)$}

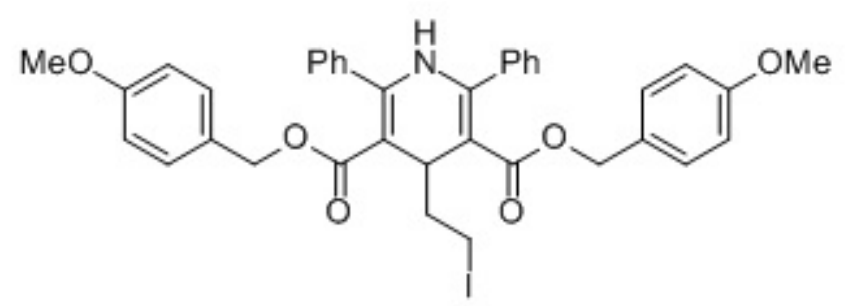

$2 p$

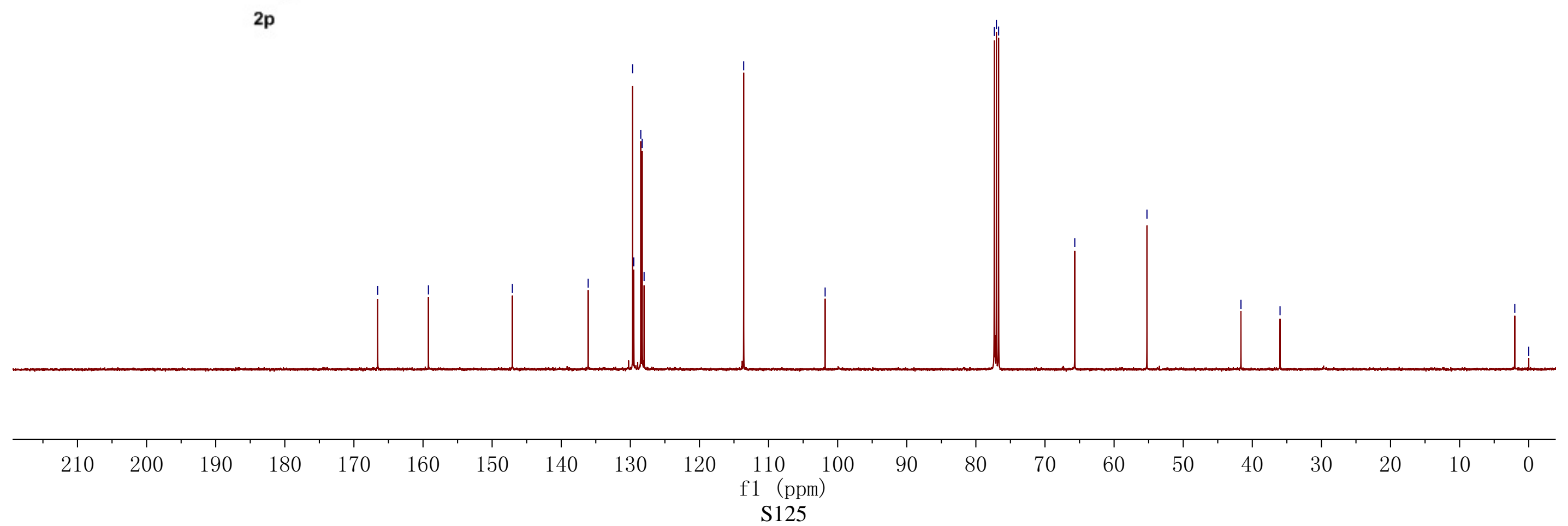


${ }^{1} \mathrm{H}$ NMR (400 MHz, $\left.\mathrm{CDCl}_{3}\right)$

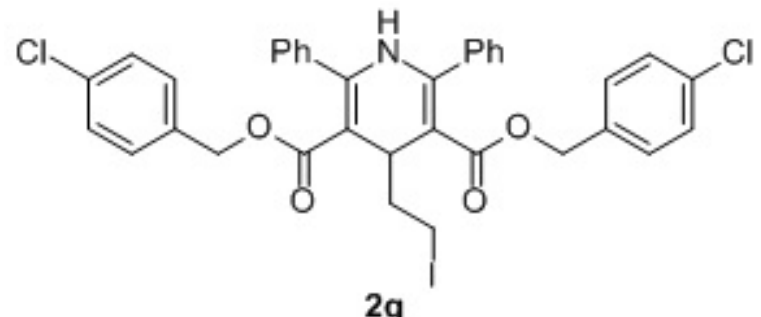

$2 q$

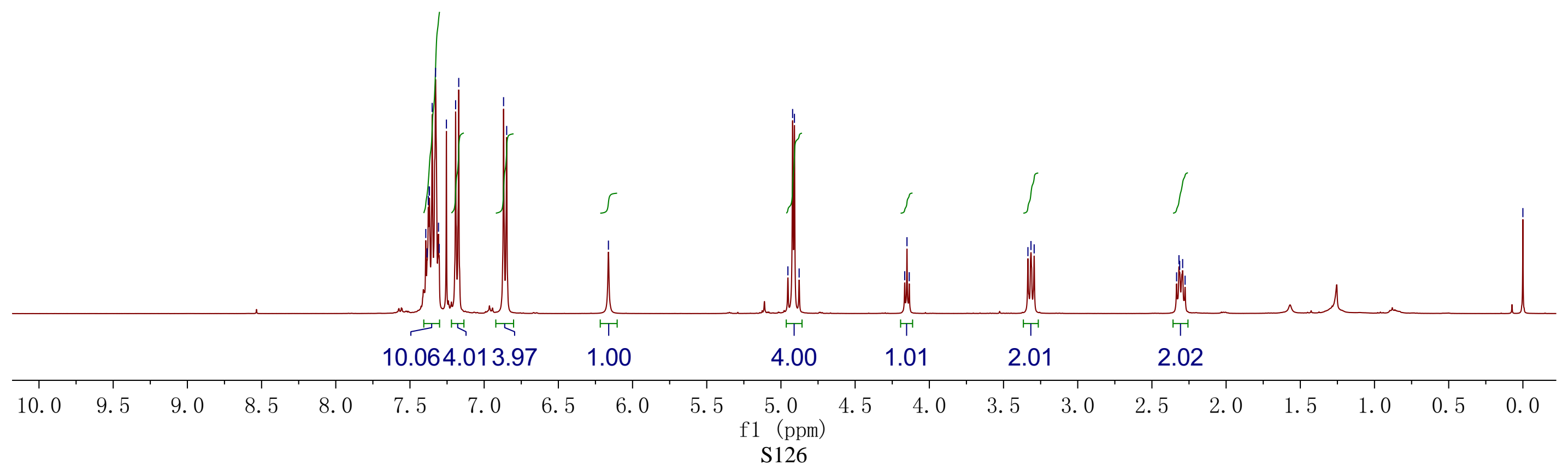


${ }^{13} \mathrm{C}$ NMR (100 MHz, $\left.\mathrm{CDCl}_{3}\right)$

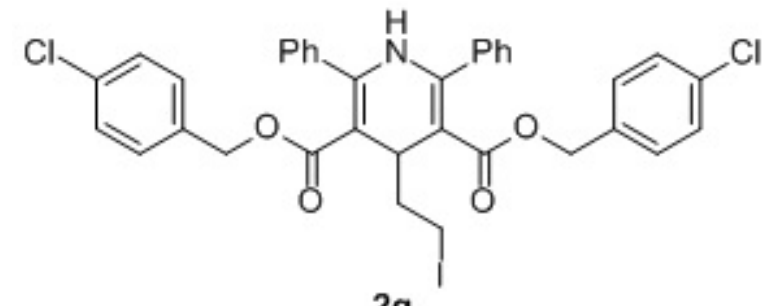

$2 q$

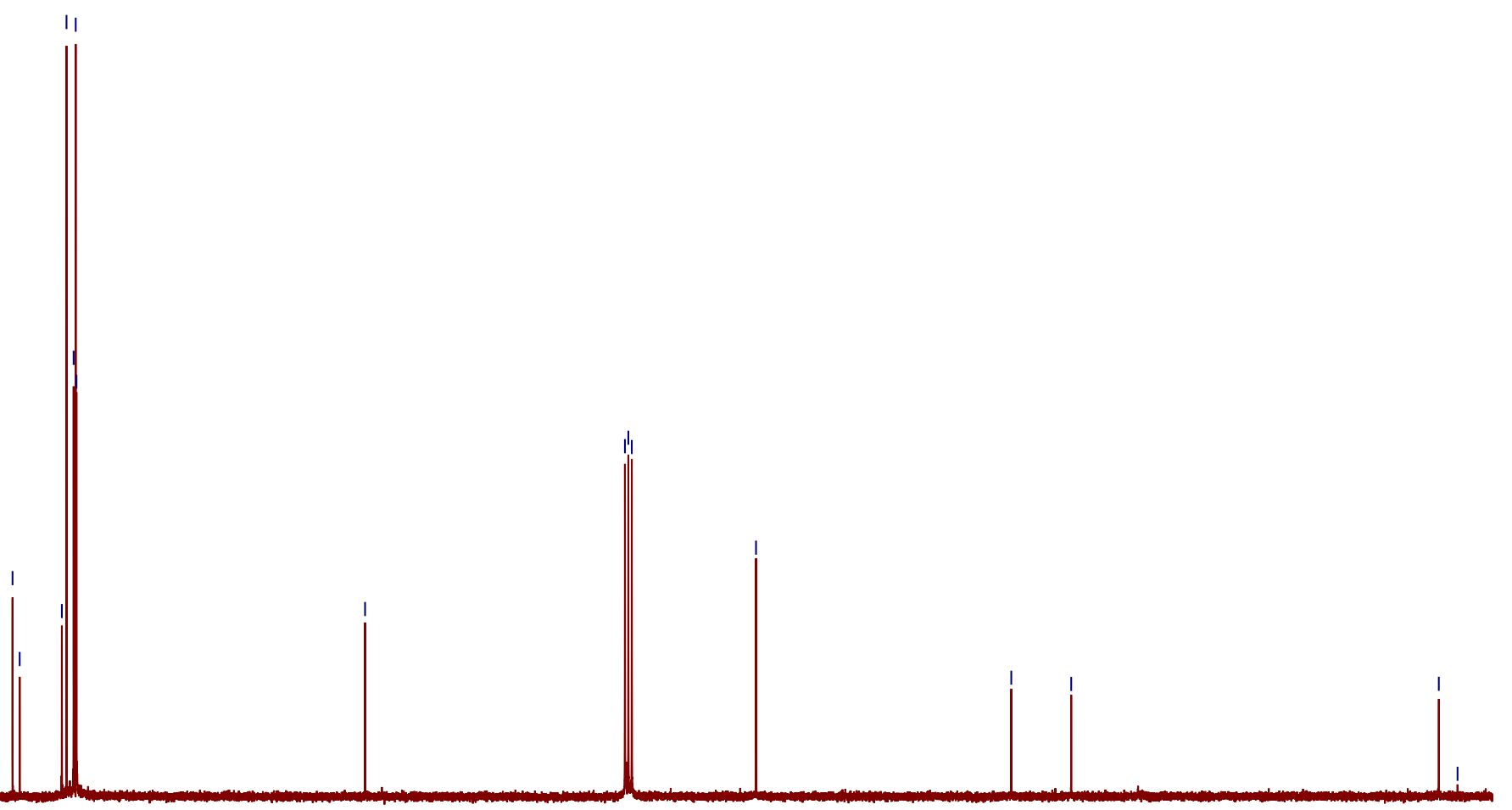


${ }^{1} \mathrm{H} \mathrm{NMR}\left(400 \mathrm{MHz}, \mathrm{CDCl}_{3}\right)$
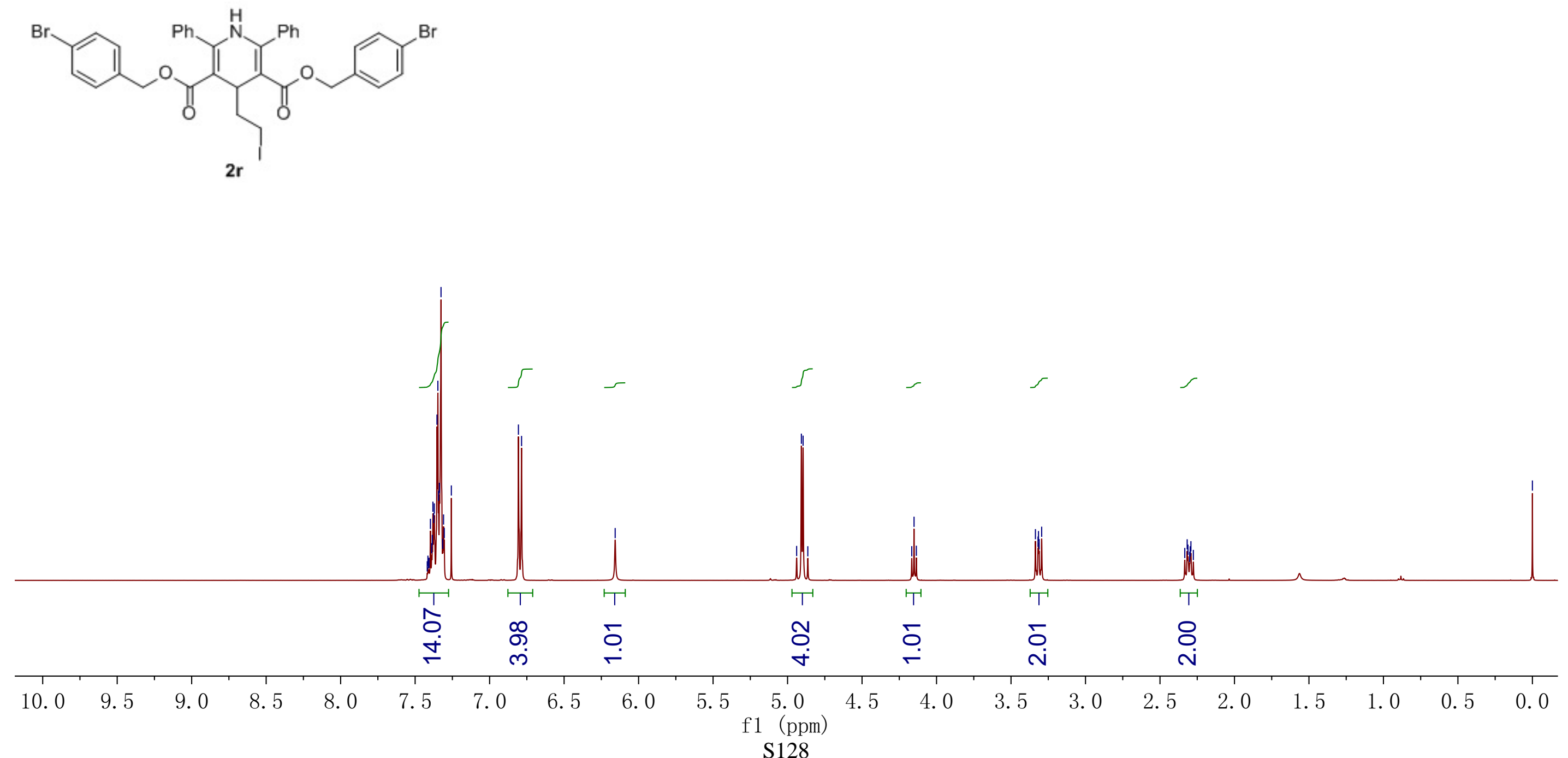


\section{${ }^{13} \mathrm{C} \mathrm{NMR}\left(100 \mathrm{MHz}, \mathrm{CDCl}_{3}\right)$}
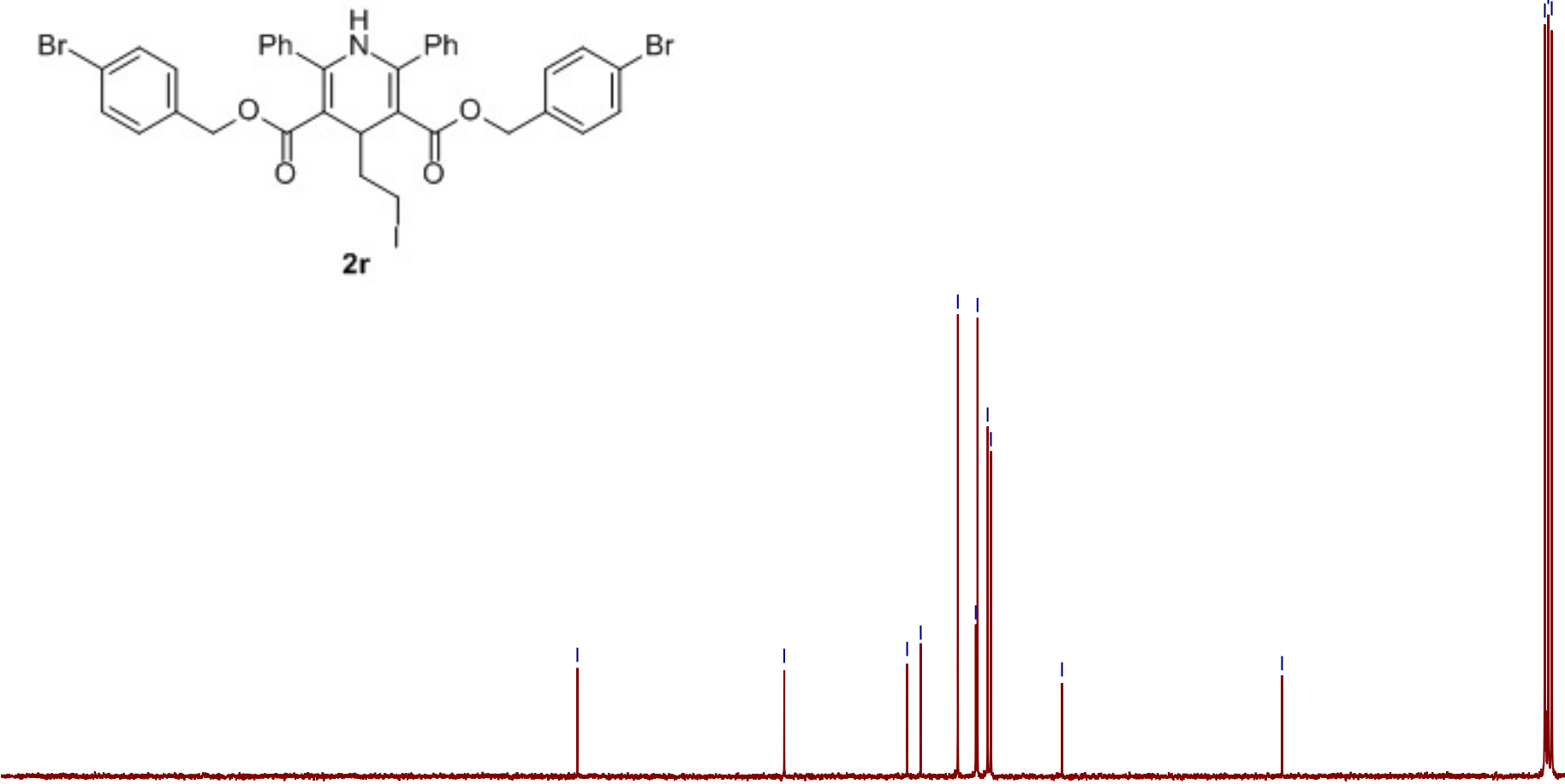
${ }^{1} \mathrm{H}$ NMR (400 MHz, $\left.\mathrm{CDCl}_{3}\right)$

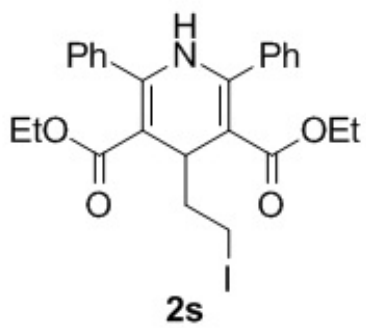

2s

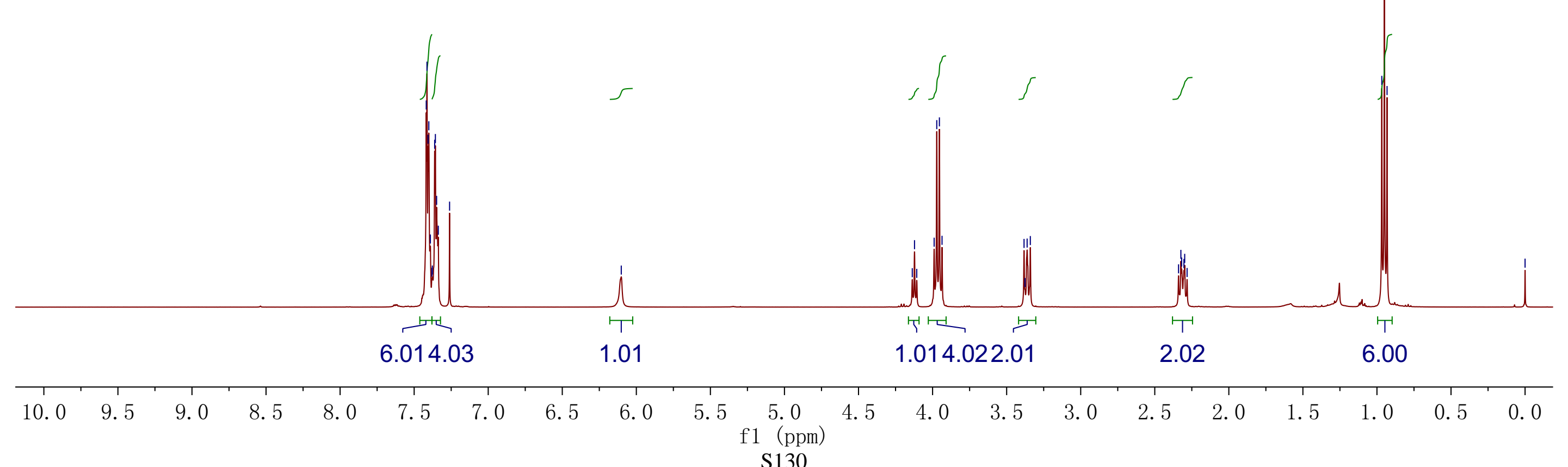


${ }^{13} \mathrm{C} \mathrm{NMR}\left(100 \mathrm{MHz}, \mathrm{CDCl}_{3}\right)$
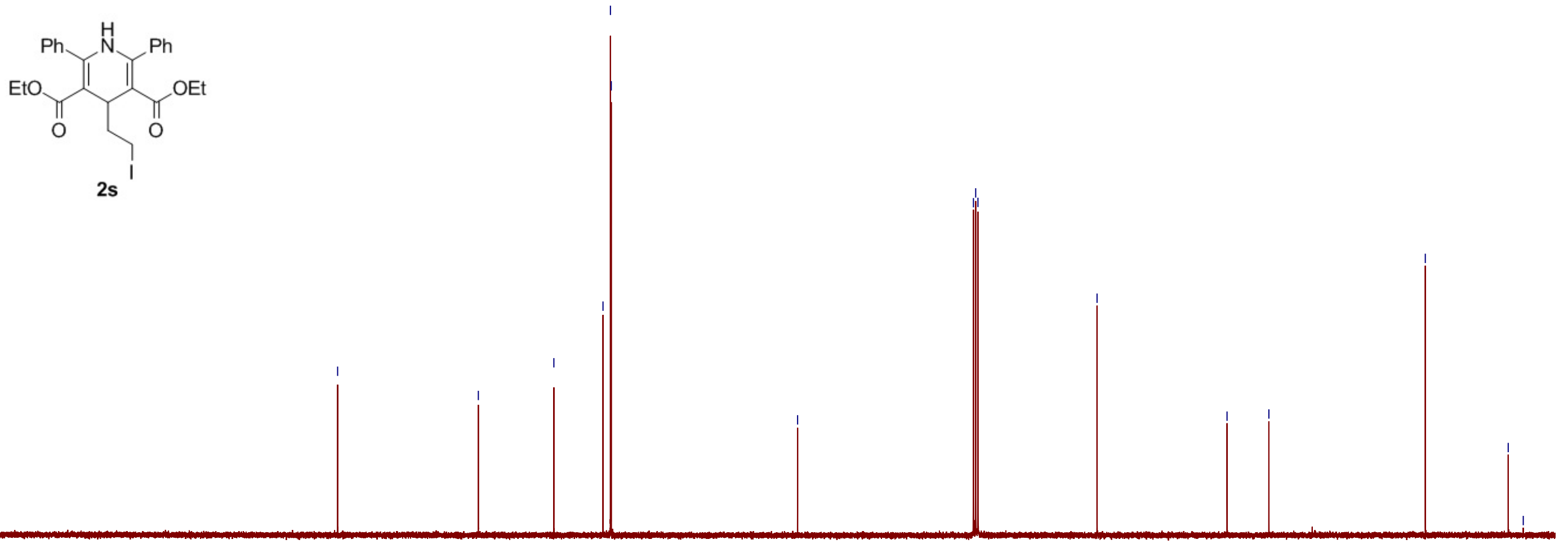

210

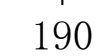




\section{${ }^{1} \mathrm{H} \mathrm{NMR}\left(400 \mathrm{MHz}, \mathrm{CDCl}_{3}\right)$}

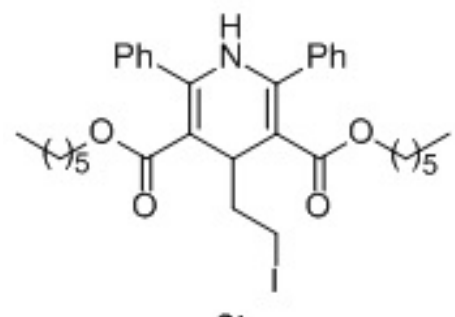

$2 \mathrm{t}$

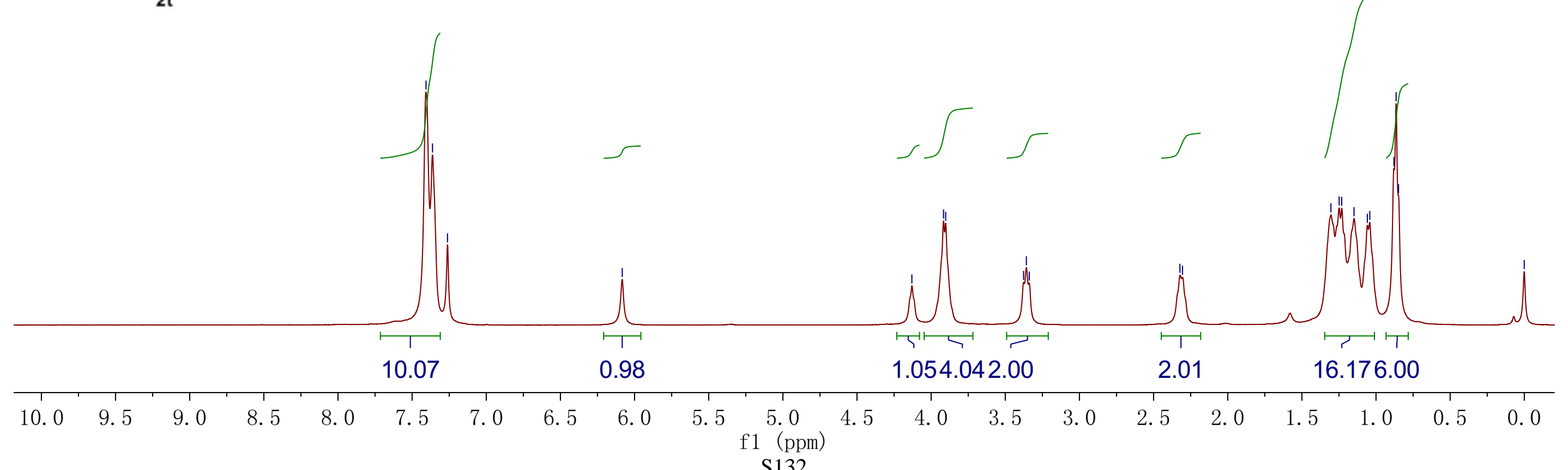


${ }^{13} \mathrm{C} \mathrm{NMR}\left(100 \mathrm{MHz}, \mathrm{CDCl}_{3}\right)$

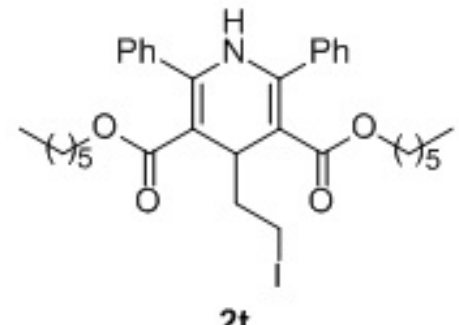

2t

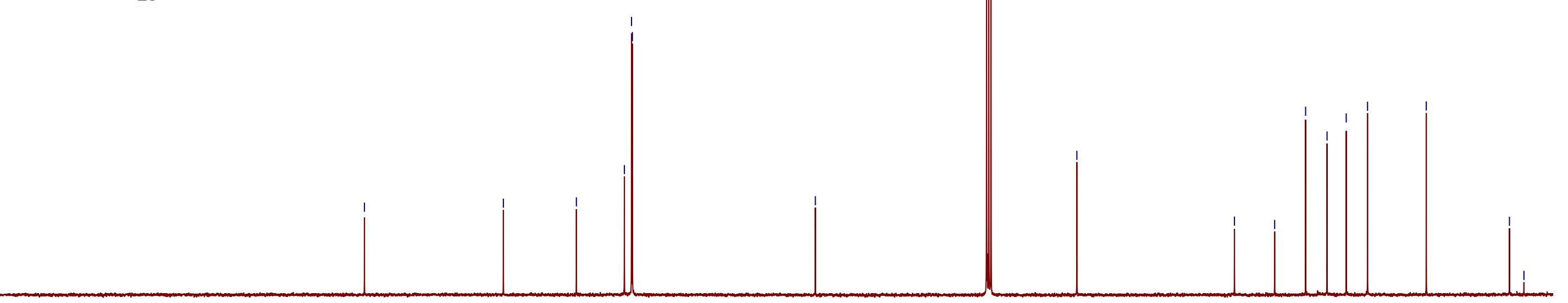

210

190 


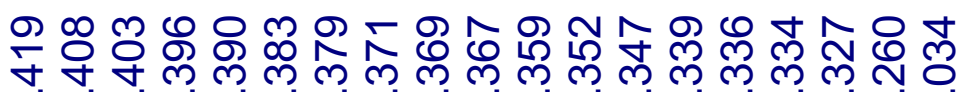

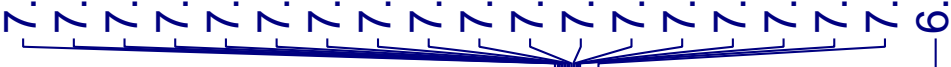
冈

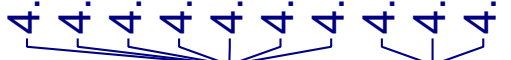

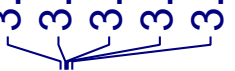

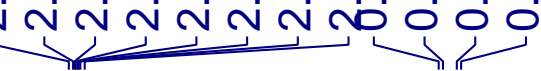

${ }^{1} \mathrm{H}$ NMR (400 MHz, $\mathrm{CDCl}_{3}$ )

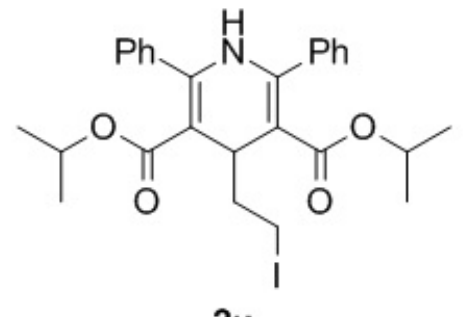

$2 \mathrm{u}$

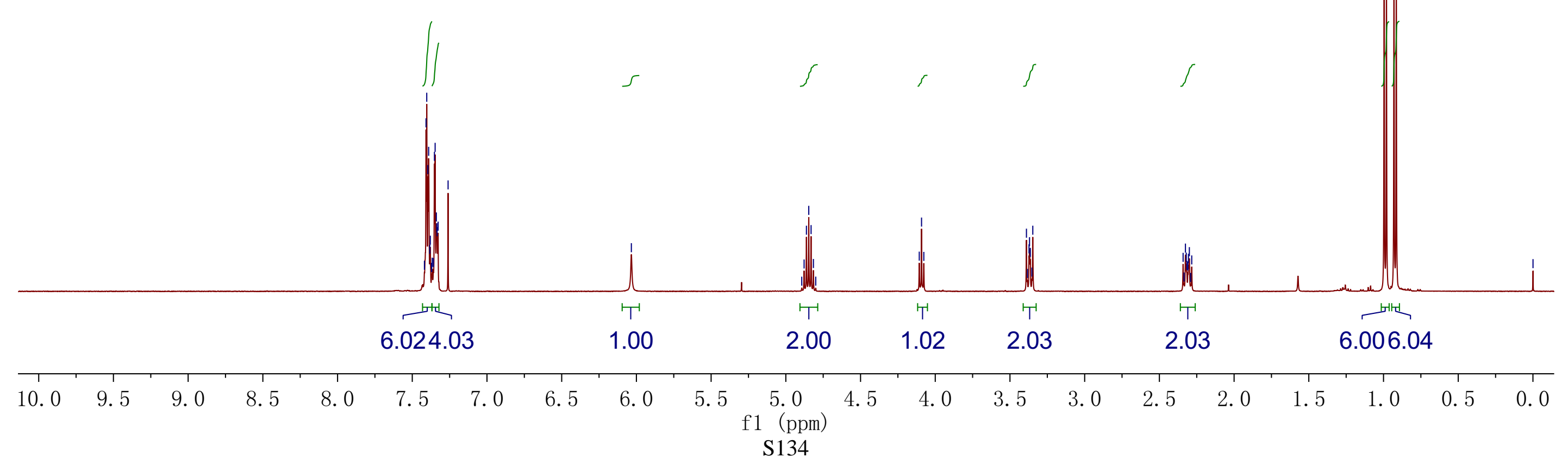


${ }^{13} \mathrm{C} \mathrm{NMR}\left(100 \mathrm{MHz}, \mathrm{CDCl}_{3}\right)$

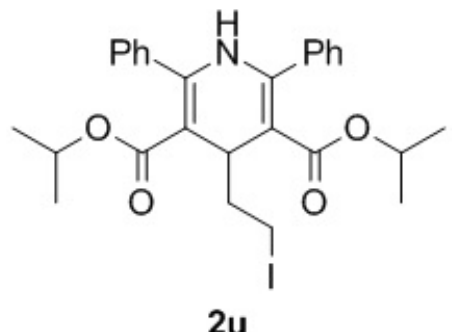

$2 u$

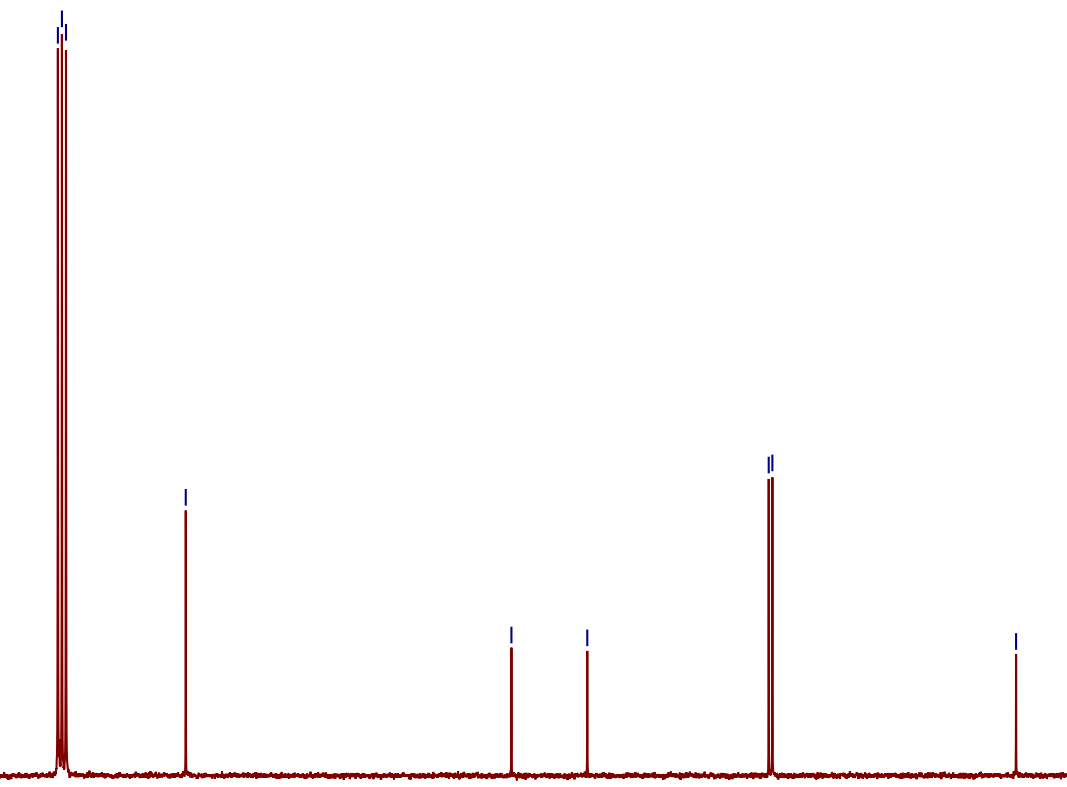


5

${ }^{1} \mathrm{H}$ NMR (400 MHz, $\mathrm{CDCl}_{3}$ )

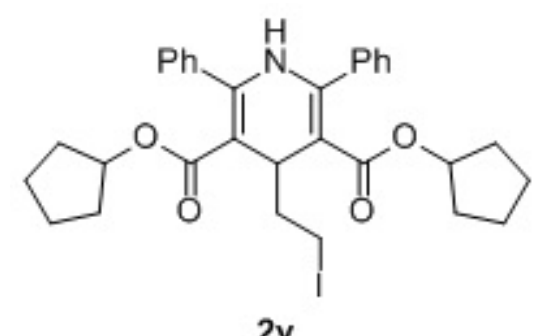

2v

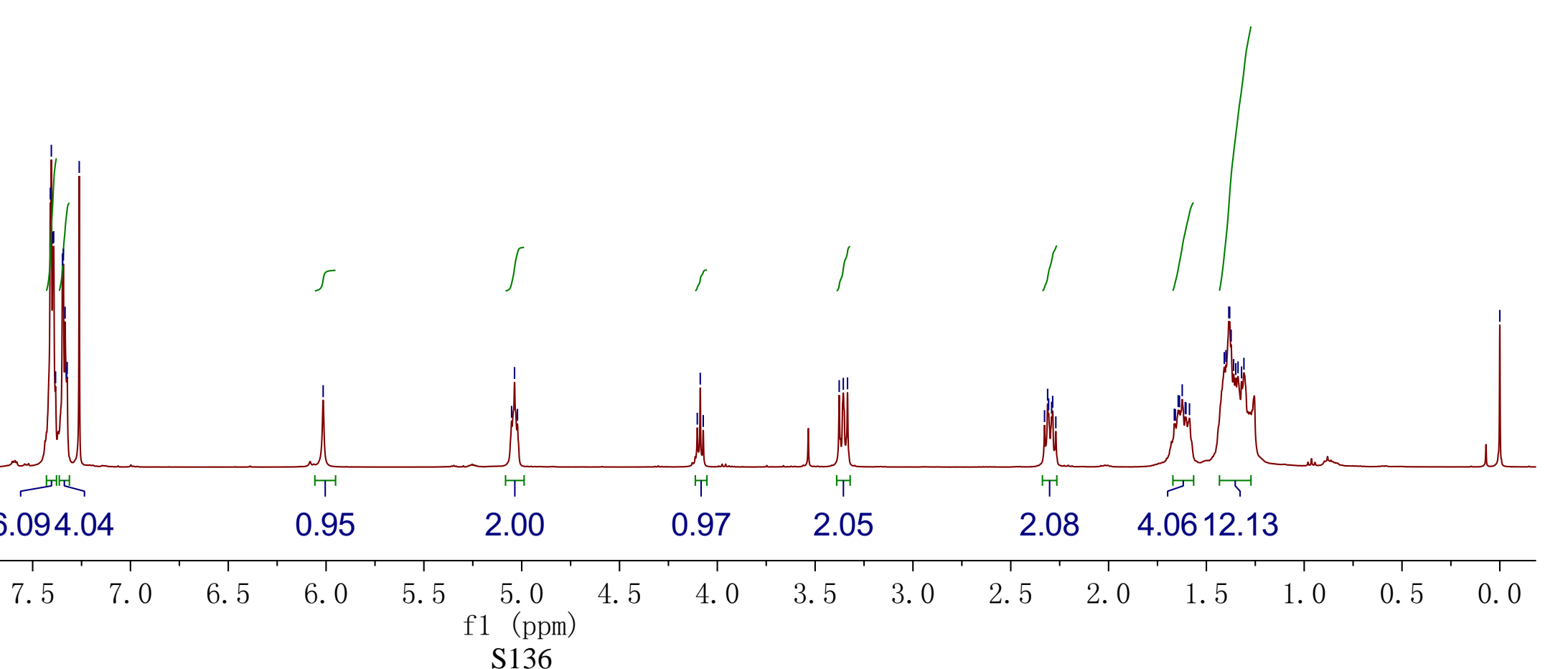


${ }^{13} \mathrm{C}$ NMR $\left(100 \mathrm{MHz}, \mathrm{CDCl}_{3}\right)$

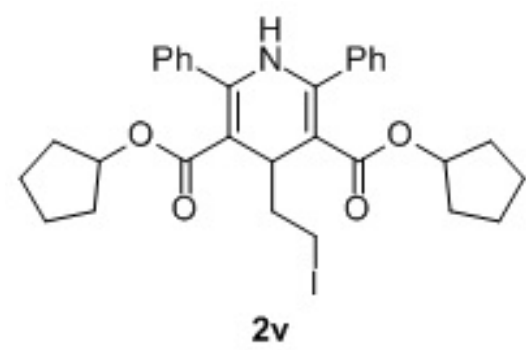

2v

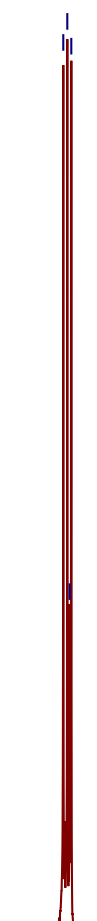

210

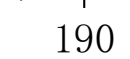

180

170

160

150

140

130

120

110

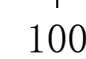

90

80

70 S137 


\section{${ }^{1} \mathrm{H}$ NMR (400 MHz, $\left.\mathrm{CDCl}_{3}\right)$}

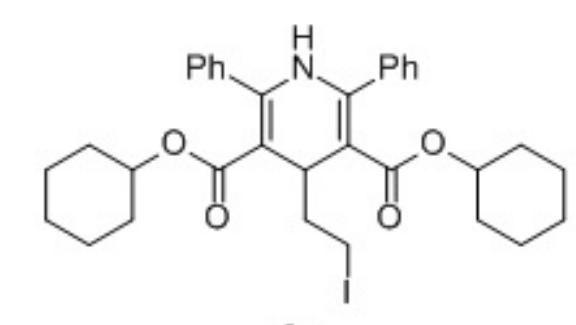

$2 w$

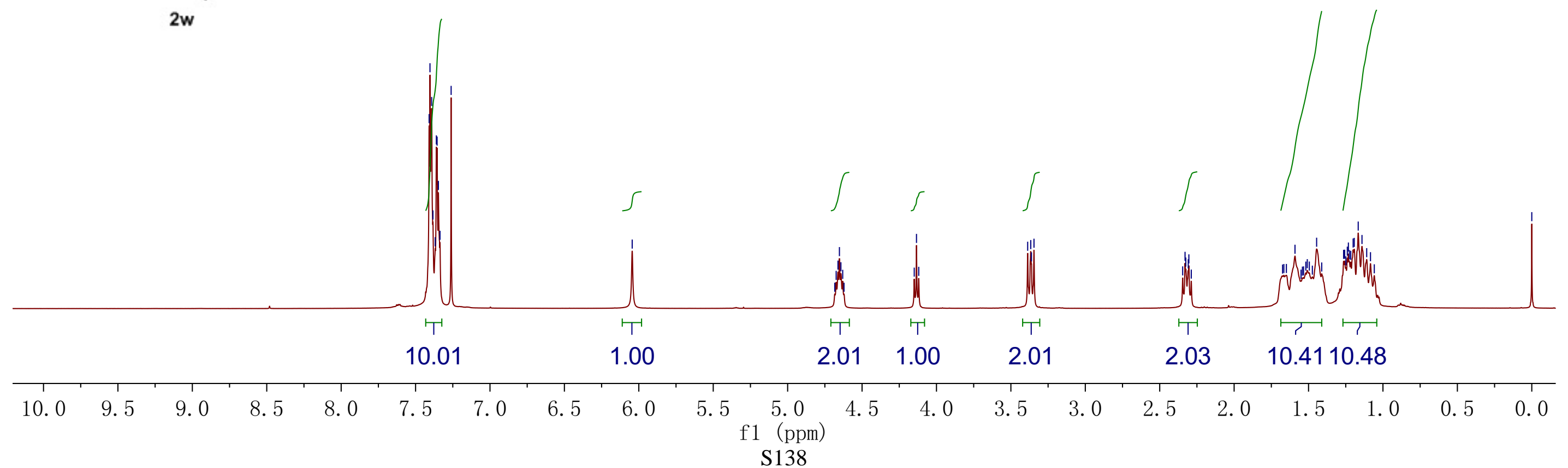




\section{${ }^{13} \mathrm{C}$ NMR (100 MHz, $\mathrm{CDCl}_{3}$ )}
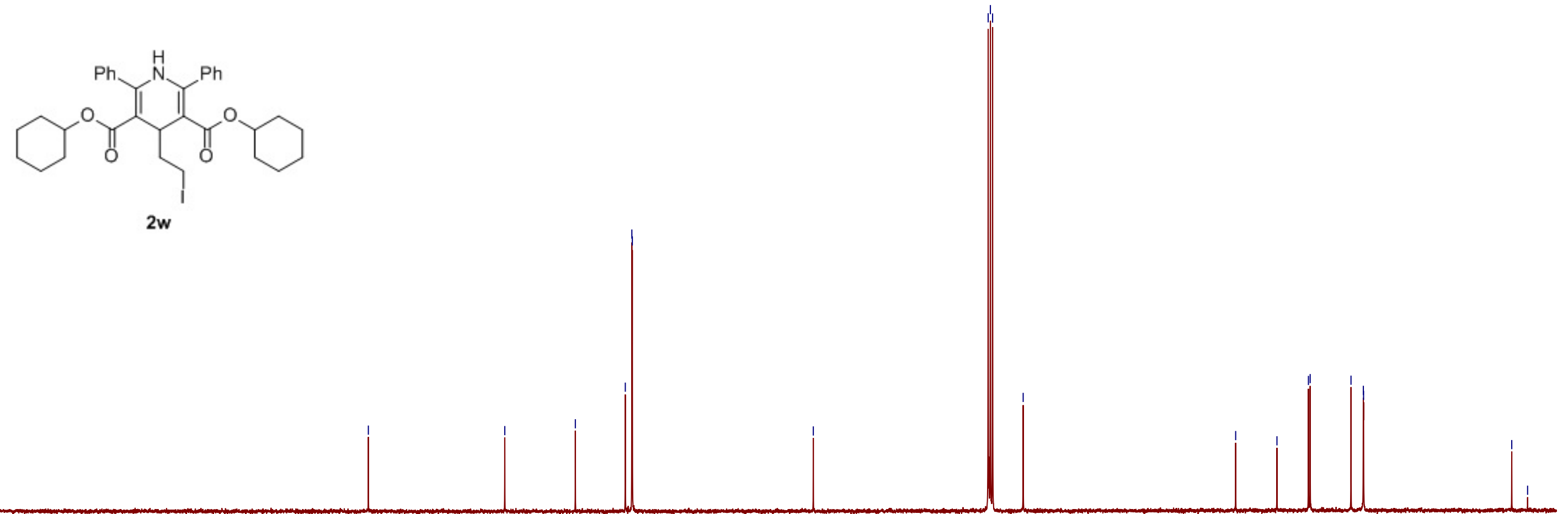

210

190 
${ }^{1} \mathrm{H}$ NMR (400 MHz, $\mathrm{CDCl}_{3}$ )
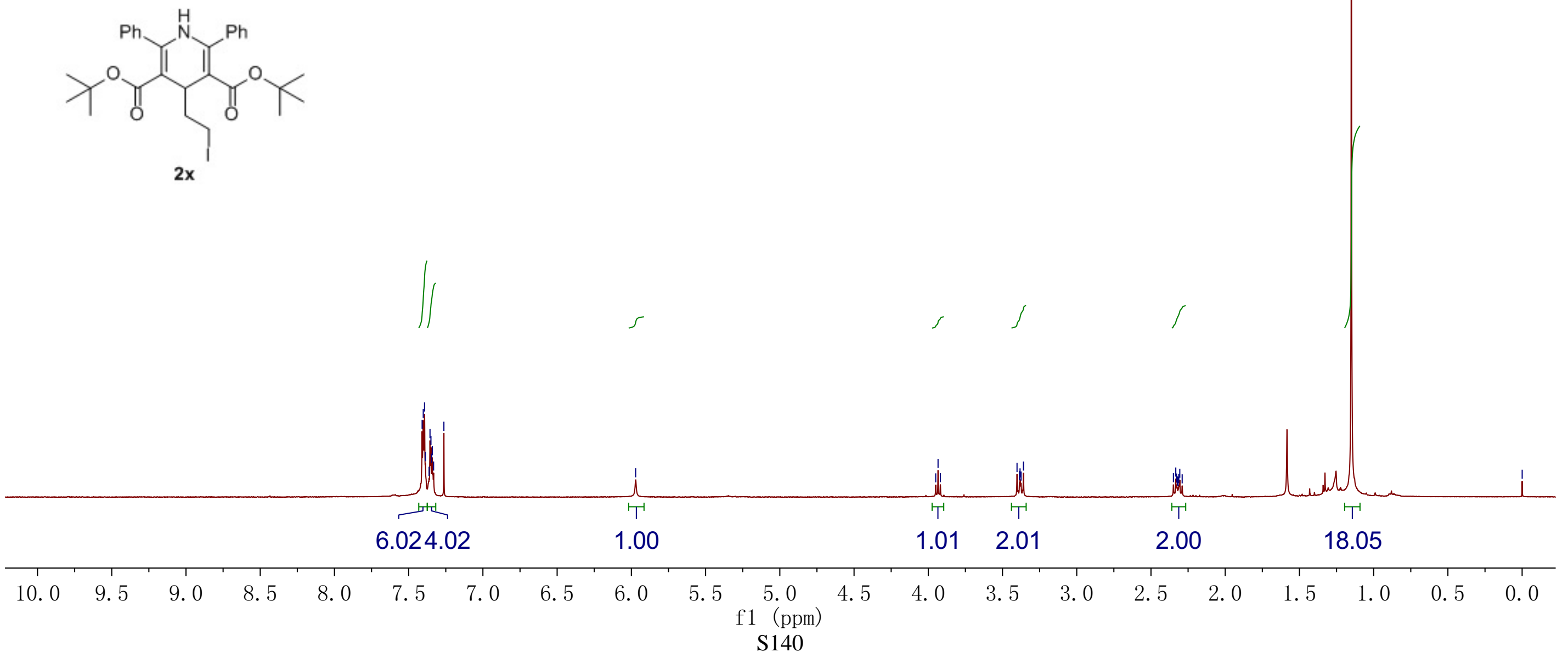


\section{${ }^{13} \mathrm{C}$ NMR (100 MHz, $\left.\mathrm{CDCl}_{3}\right)$}
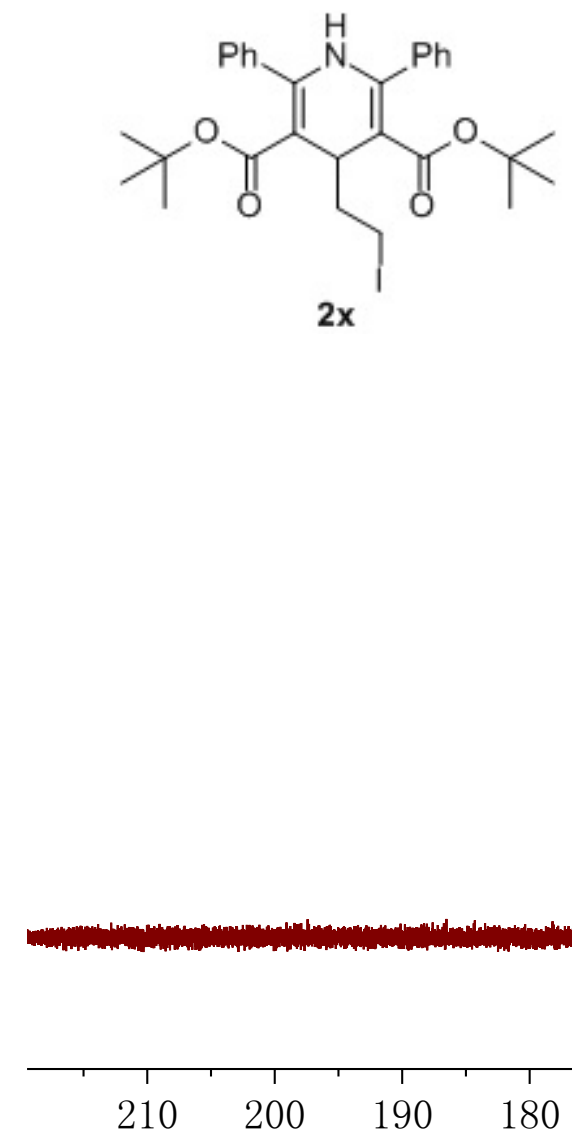
${ }^{1} \mathrm{H}$ NMR (400 MHz, $\left.\mathrm{CDCl}_{3}\right)$
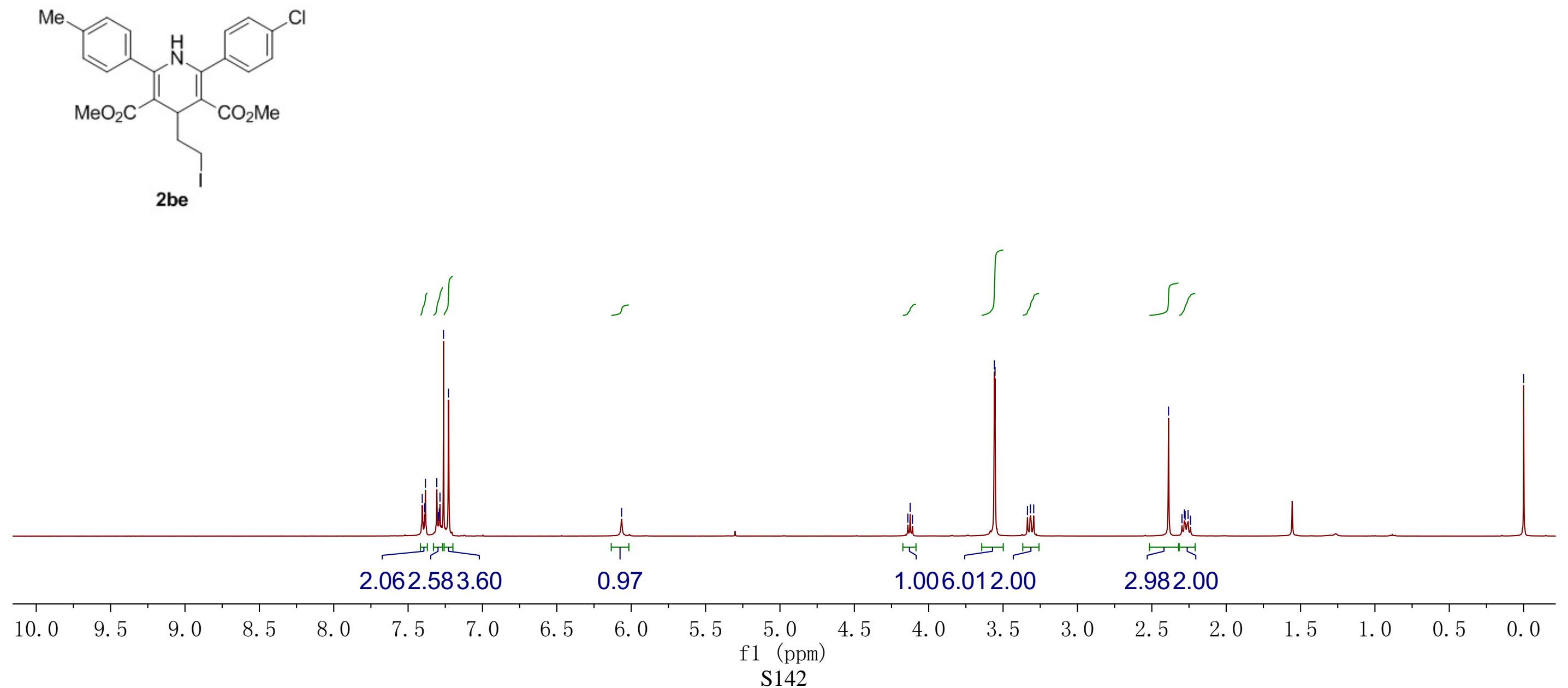
${ }^{13} \mathrm{C} \mathrm{NMR}\left(100 \mathrm{MHz}, \mathrm{CDCl}_{3}\right)$

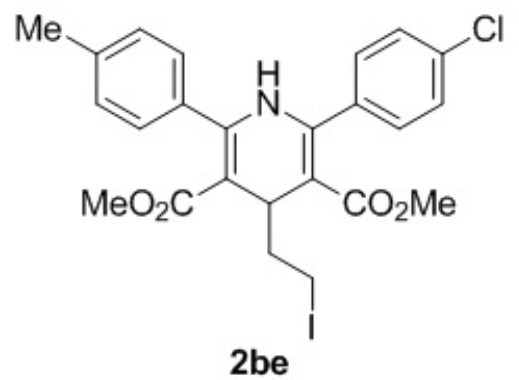

2be

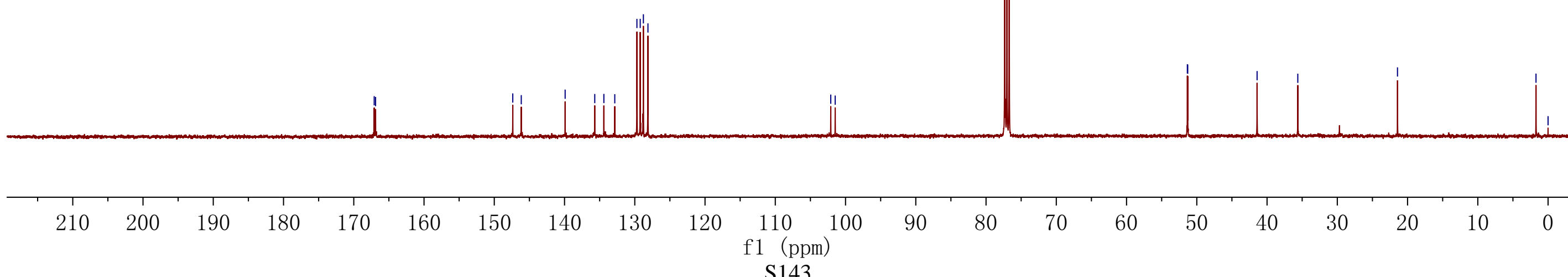


${ }^{1} \mathrm{H} \mathrm{NMR}\left(400 \mathrm{MHz}, \mathrm{CDCl}_{3}\right)$
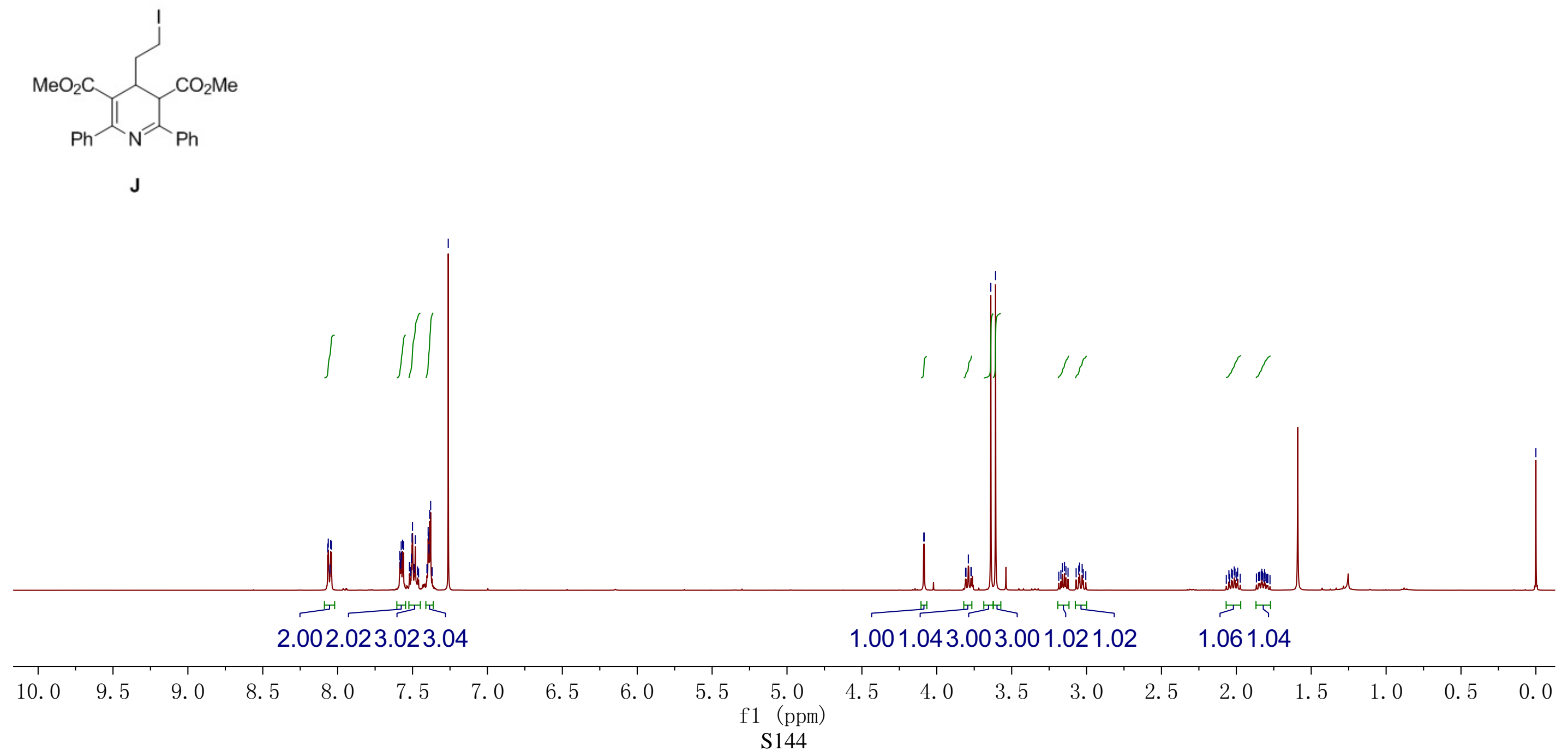


\section{${ }^{13} \mathrm{C}$ NMR (100 MHz, $\left.\mathrm{CDCl}_{3}\right)$}
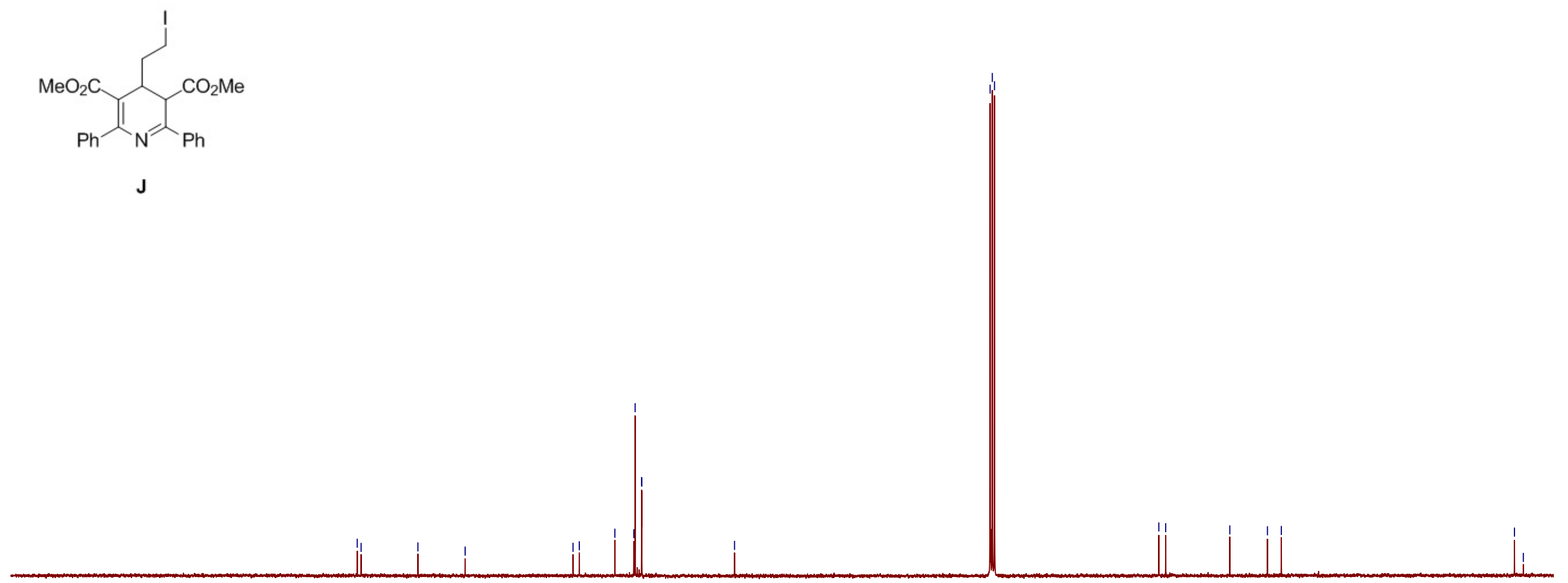
${ }^{1} \mathrm{H}$ NMR (400 MHz, $\mathrm{CDCl}_{3}$ )
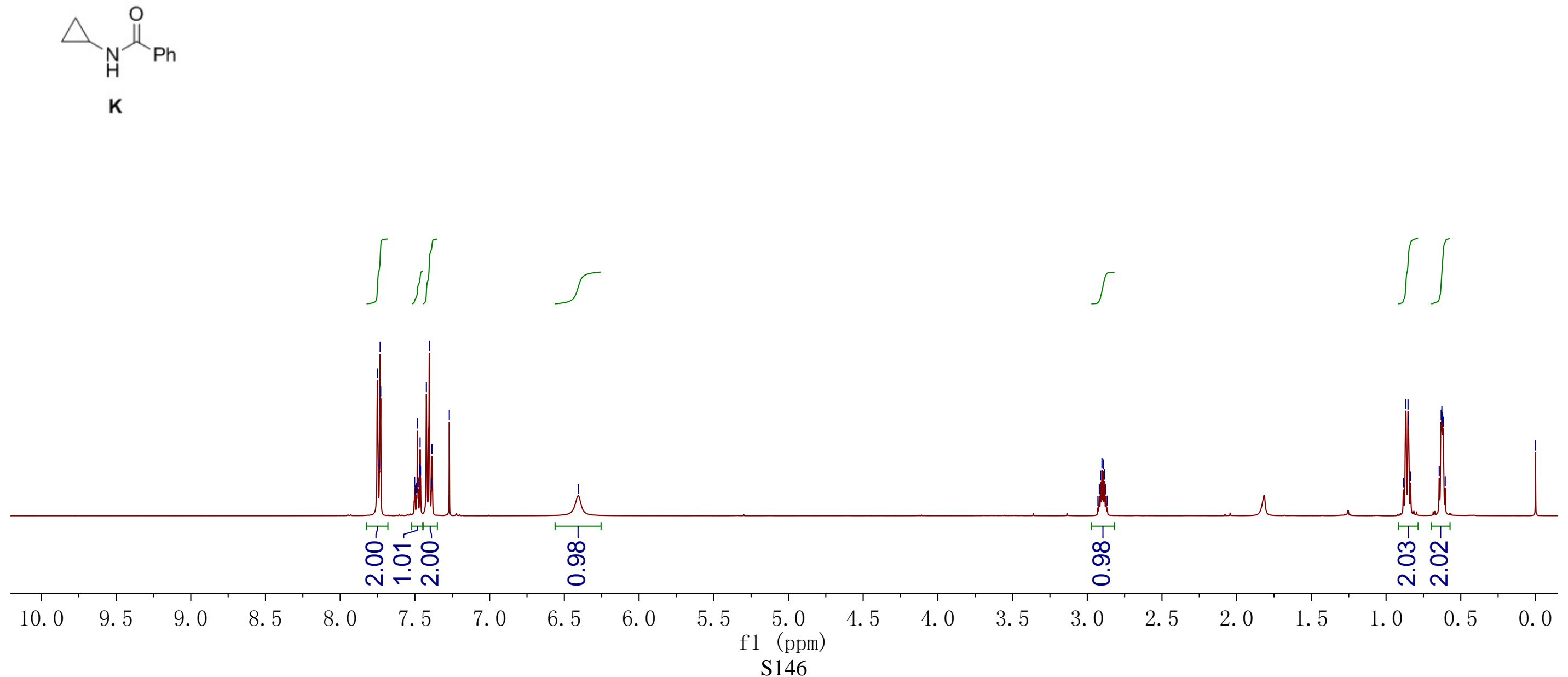

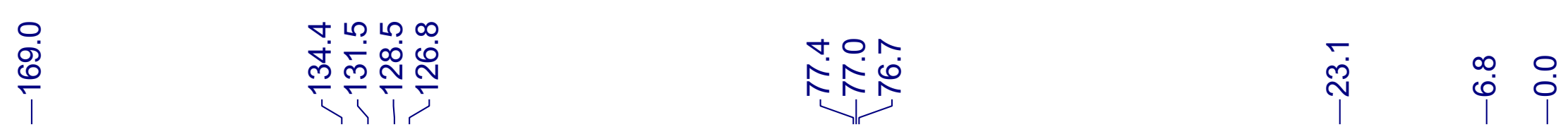

${ }^{13} \mathrm{C}$ NMR (100 MHz, $\left.\mathrm{CDCl}_{3}\right)$
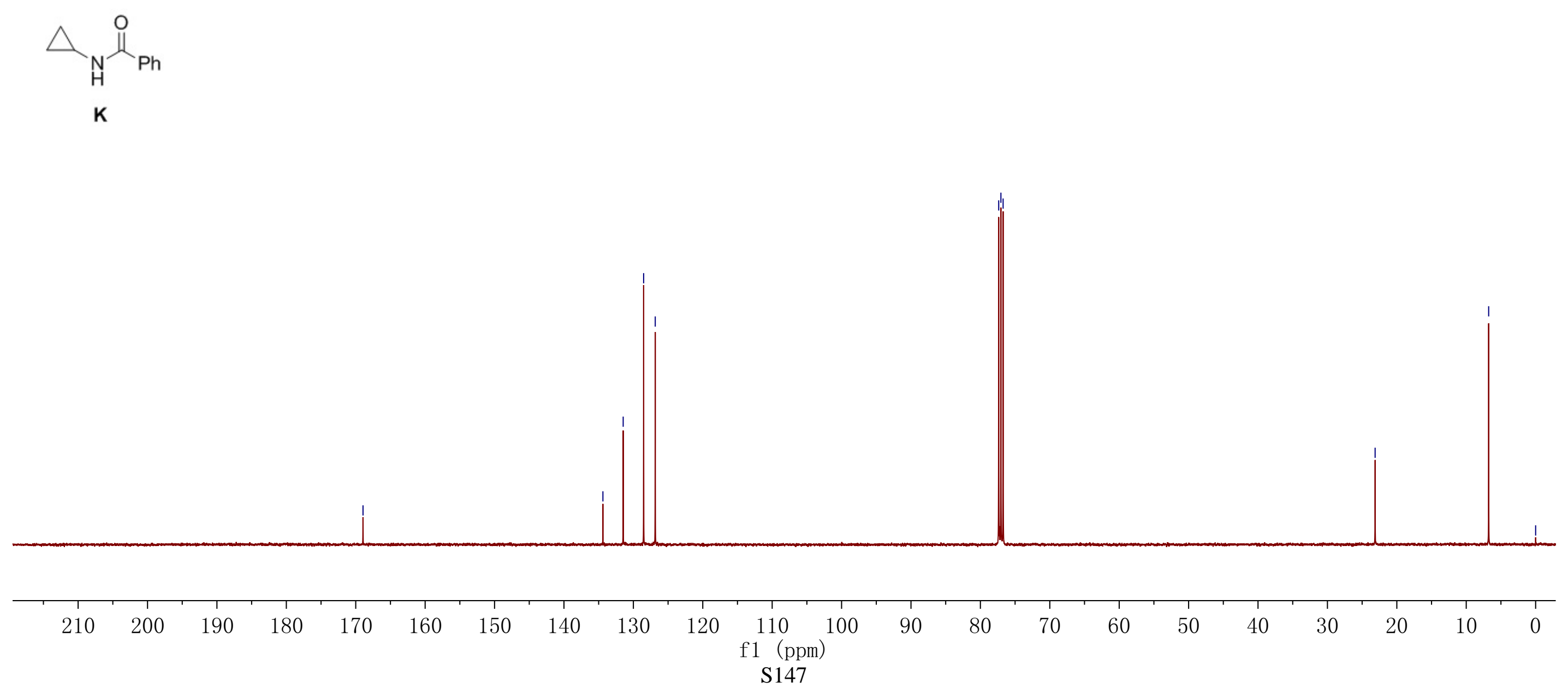
${ }^{1} \mathrm{H}$ NMR (400 MHz, $\mathrm{CDCl}_{3}$ )

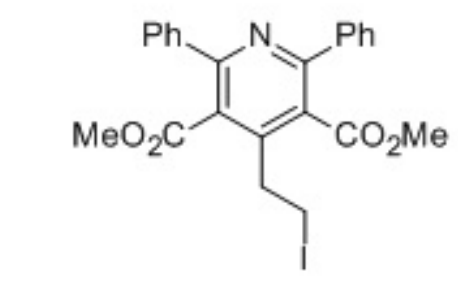

$3 a$

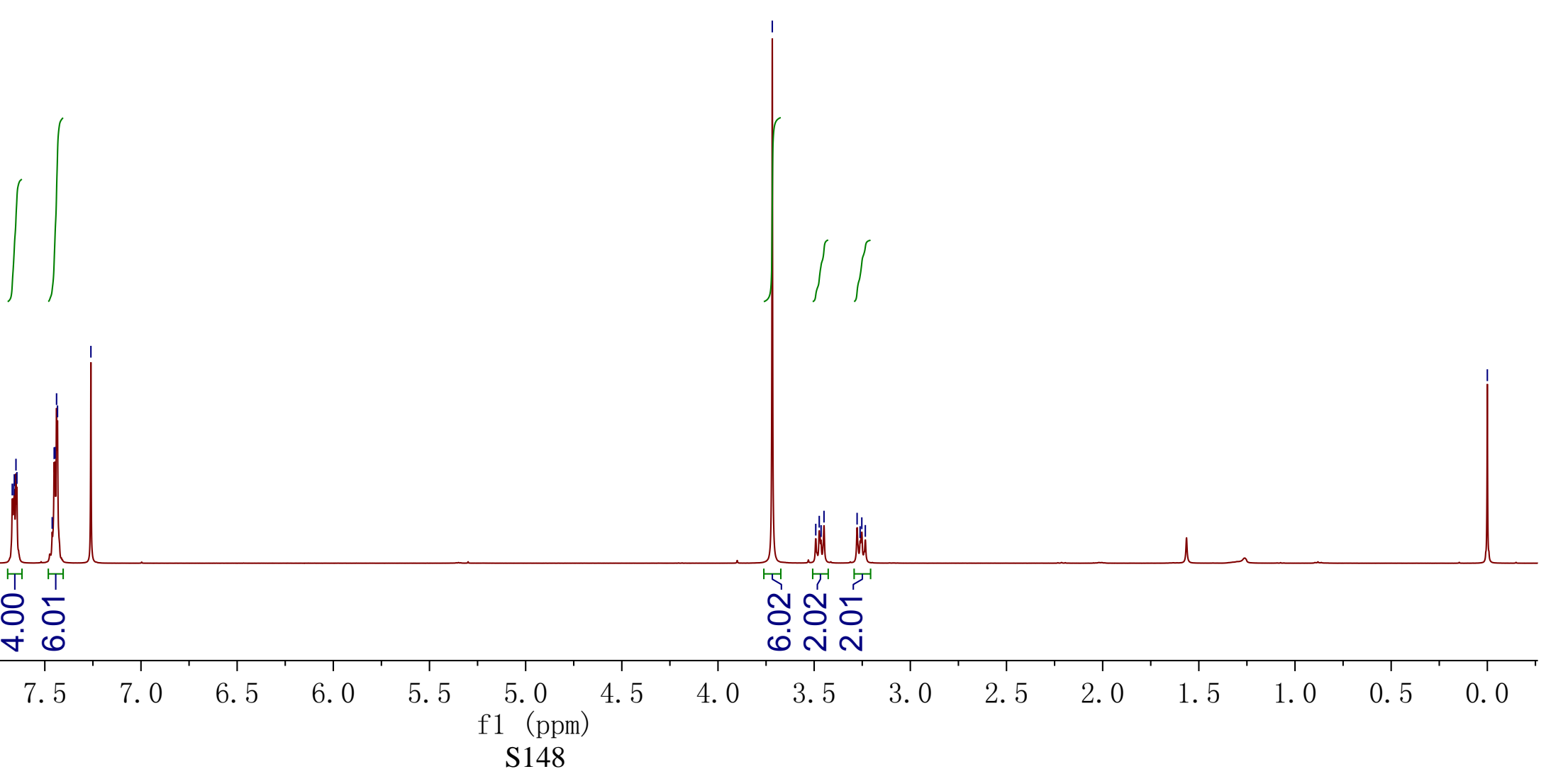


${ }^{13} \mathrm{C}$ NMR (100 MHz, $\left.\mathrm{CDCl}_{3}\right)$

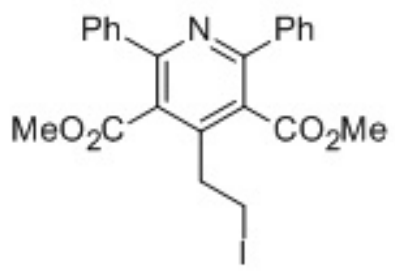

3a 
${ }^{1} \mathrm{H}$ NMR (400 MHz, $\left.\mathrm{CDCl}_{3}\right)$

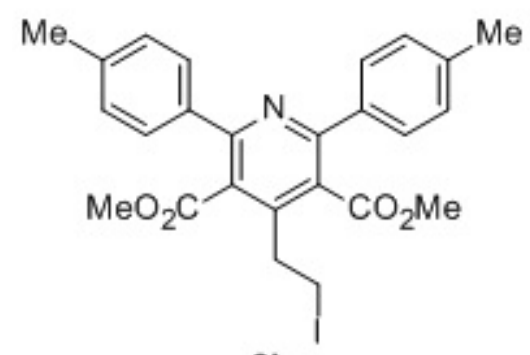

3b

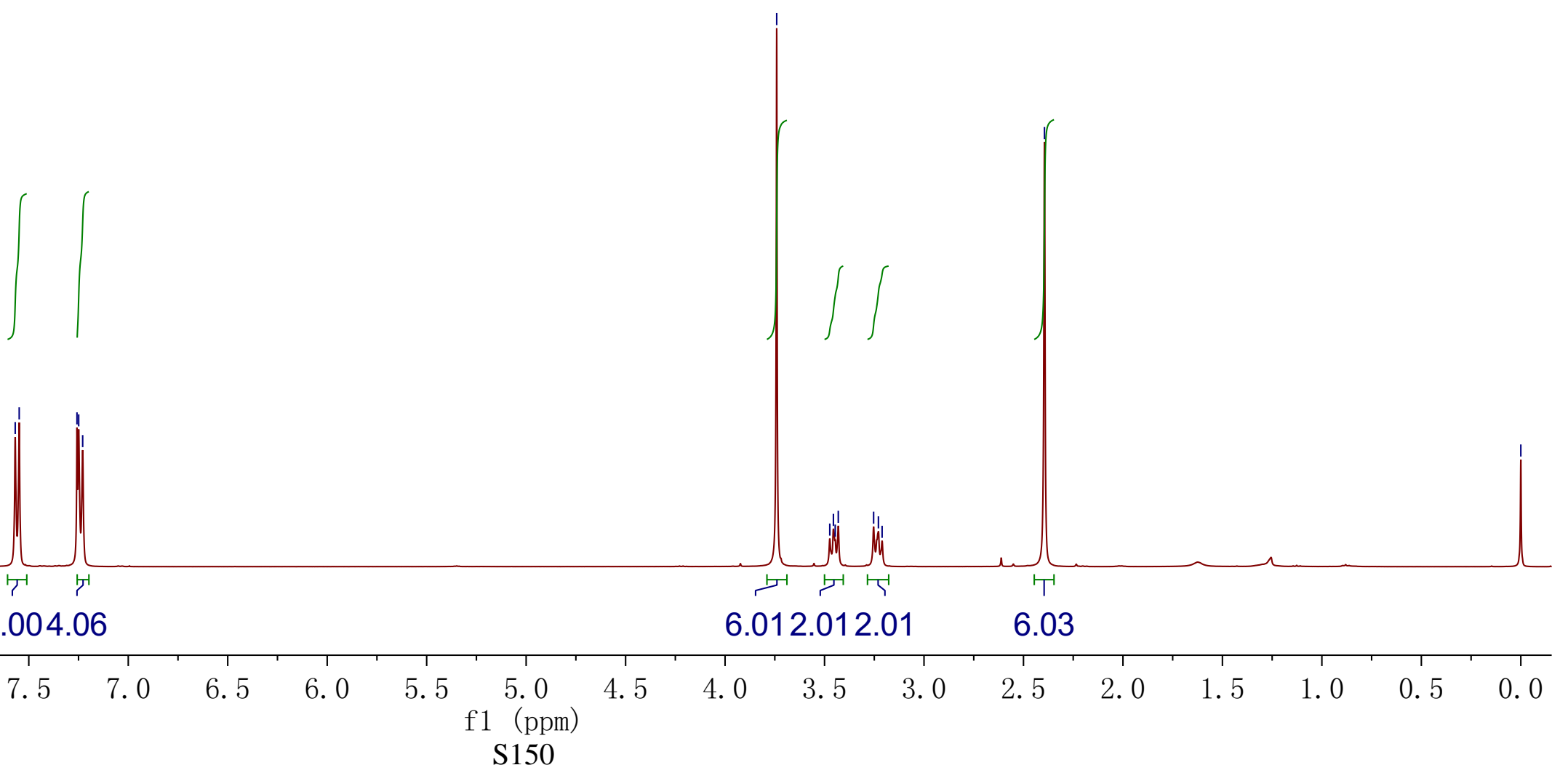


${ }^{13} \mathrm{C}$ NMR $\left(100 \mathrm{MHz}, \mathrm{CDCl}_{3}\right)$

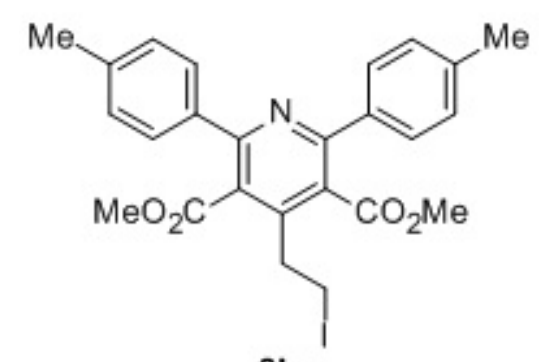

3b

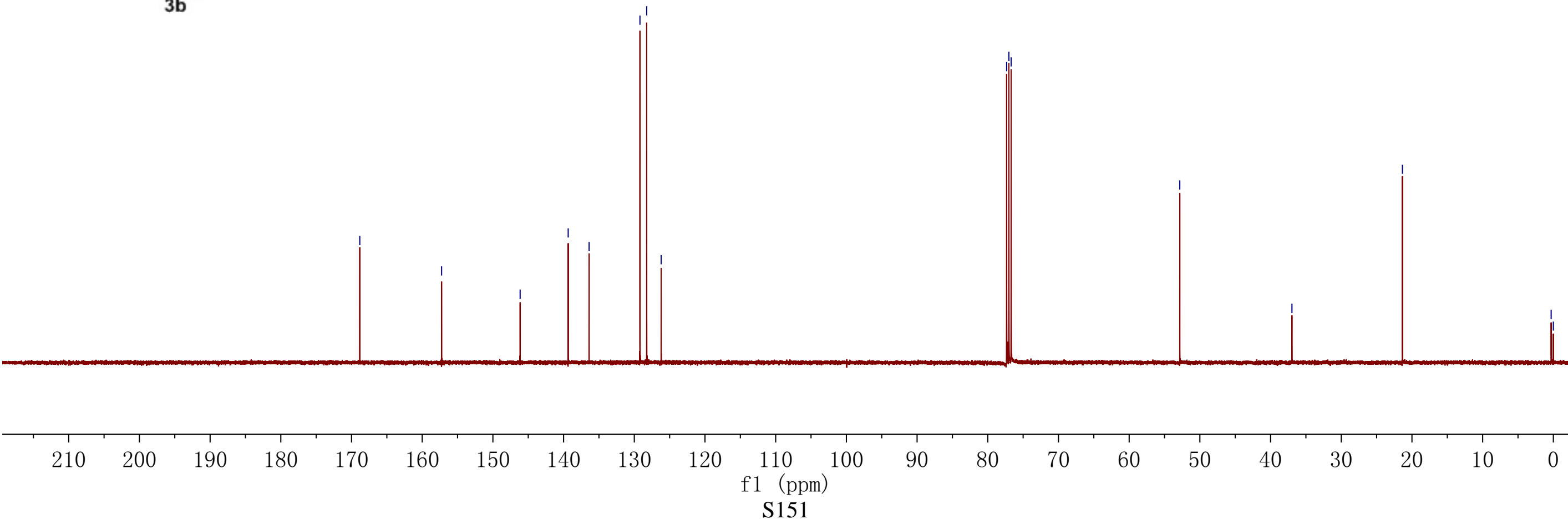


${ }^{1} \mathrm{H}$ NMR (400 MHz, $\mathrm{CDCl}_{3}$ )
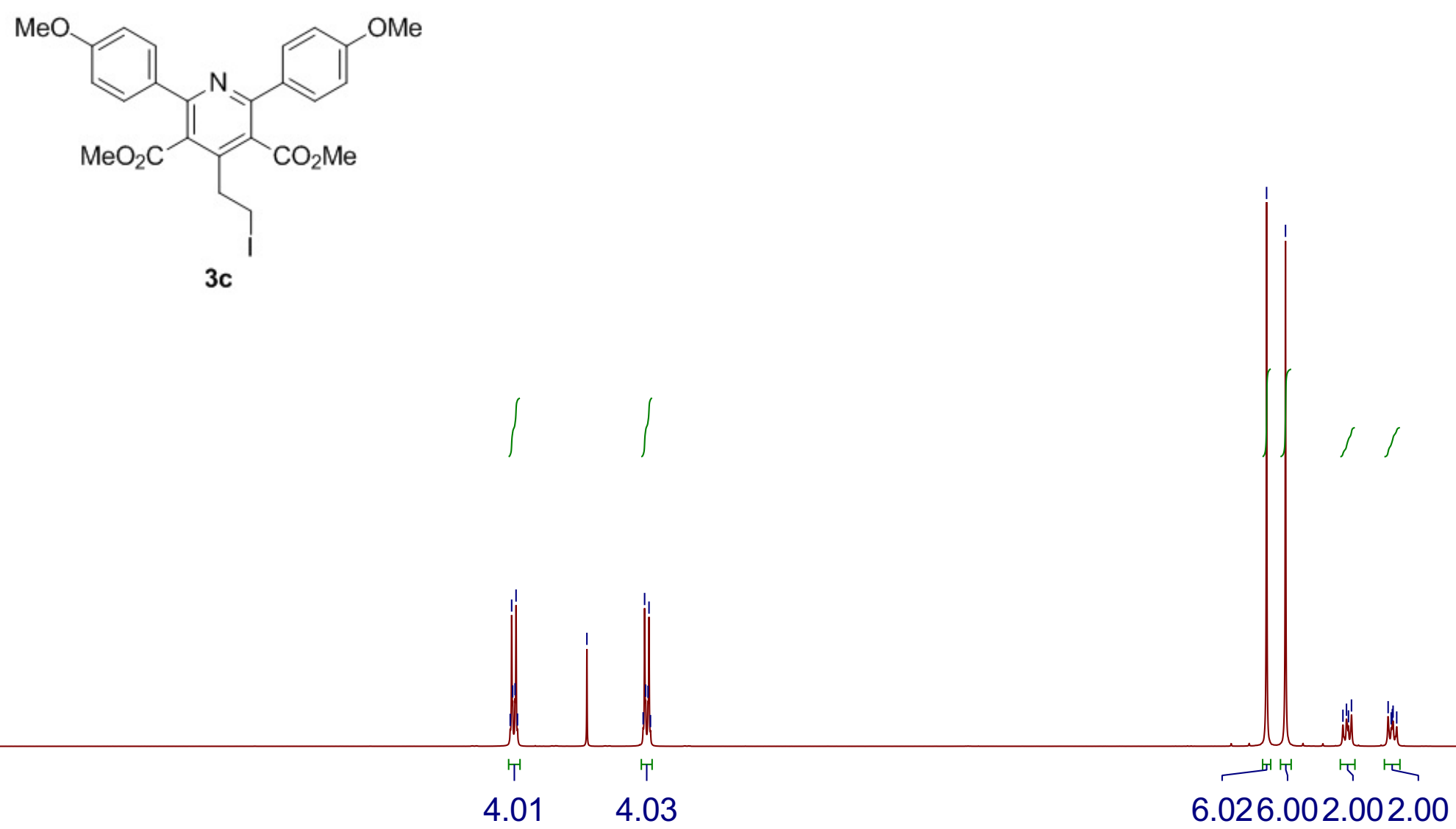

\begin{tabular}{|c|c|c|c|c|c|c|c|c|c|c|c|c|c|c|c|c|c|c|c|c|}
\hline 10.0 & 9.5 & 9.0 & 8.5 & 8.0 & 7.5 & 7. 0 & 6.5 & 6.0 & 5.5 & $\begin{array}{r}5.0 \\
1 \text { (pp }\end{array}$ & 4.5 & 4. 0 & 3.5 & 3.0 & 2.5 & 2.0 & 1.5 & 1.0 & 0.5 & 0.0 \\
\hline
\end{tabular}




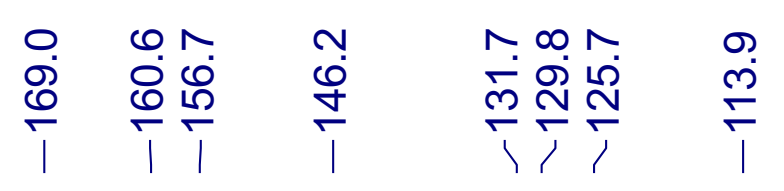

${ }^{13} \mathrm{C} \mathrm{NMR}\left(100 \mathrm{MHz}, \mathrm{CDCl}_{3}\right)$

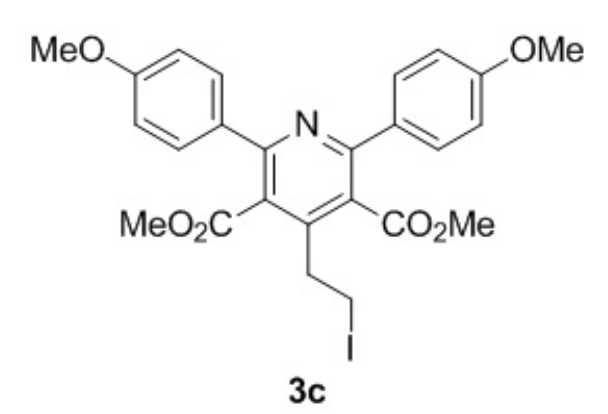

กำ

소 m.

กิ

11

$\stackrel{\circ}{\text { ก. }}$ 
${ }^{1} \mathrm{H} \mathrm{NMR}\left(400 \mathrm{MHz}, \mathrm{CDCl}_{3}\right)$

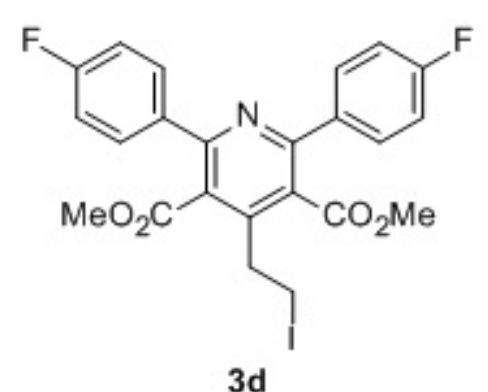

3d

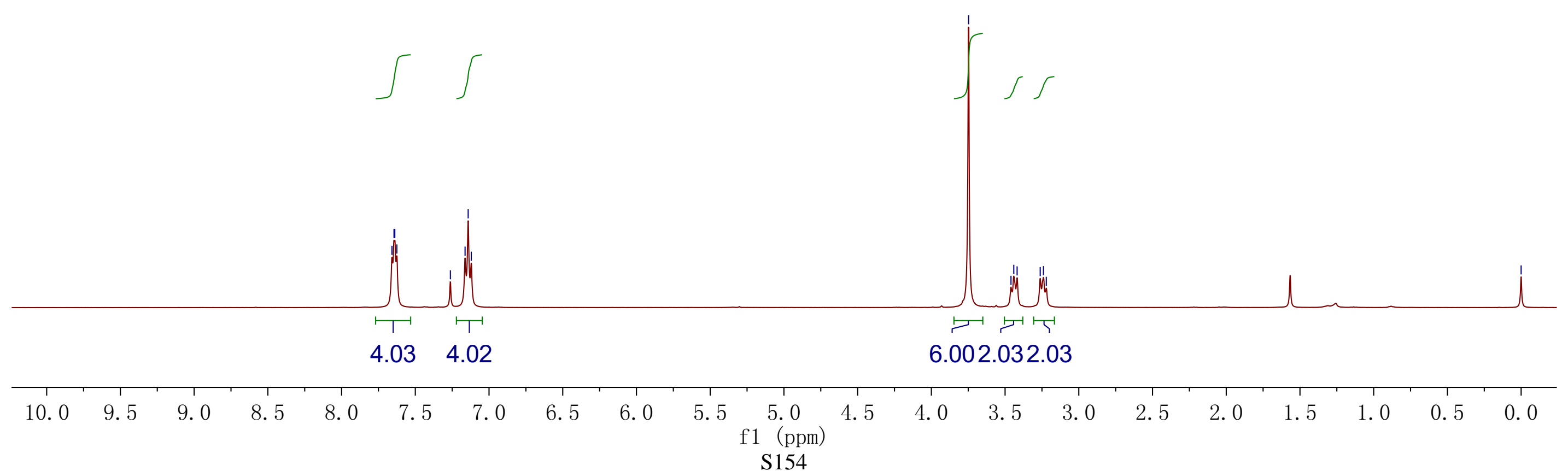




\section{${ }^{13} \mathrm{C}$ NMR (100 MHz, $\left.\mathrm{CDCl}_{3}\right)$}

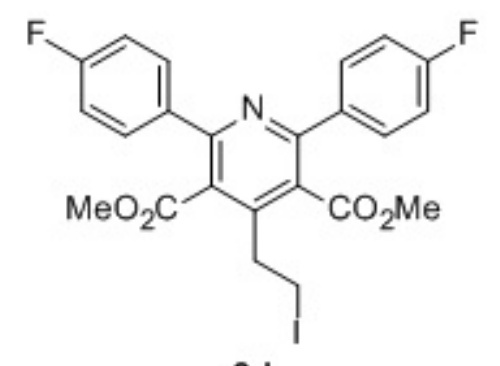

3d

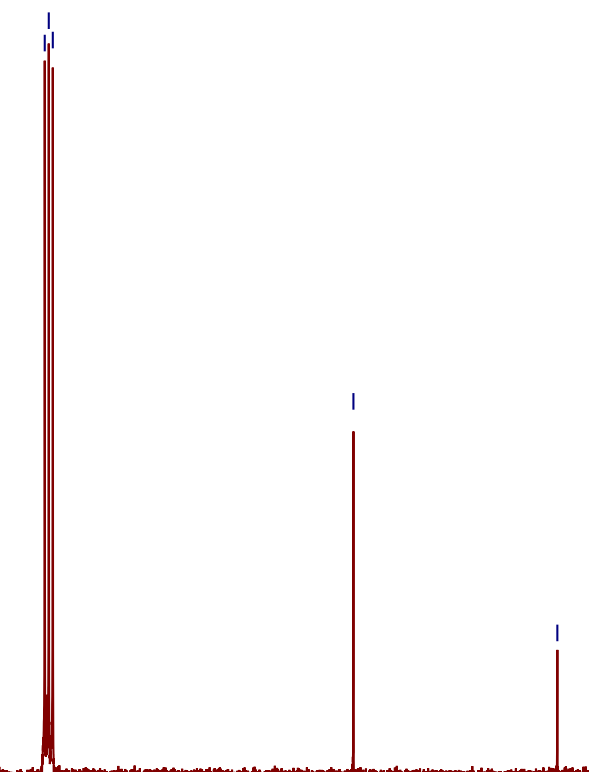


${ }^{19} \mathrm{~F}$ NMR (376 $\mathrm{MHz}, \mathrm{CDCl}_{3}$ )

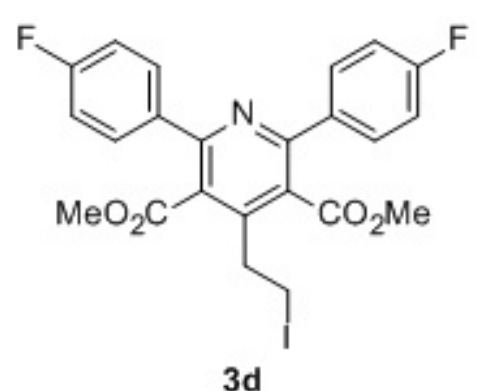

3d

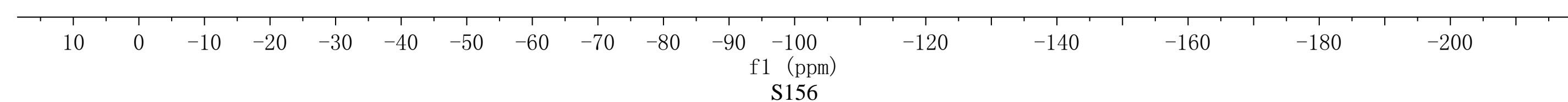


${ }^{1} \mathrm{H} \mathrm{NMR}\left(400 \mathrm{MHz}, \mathrm{CDCl}_{3}\right)$
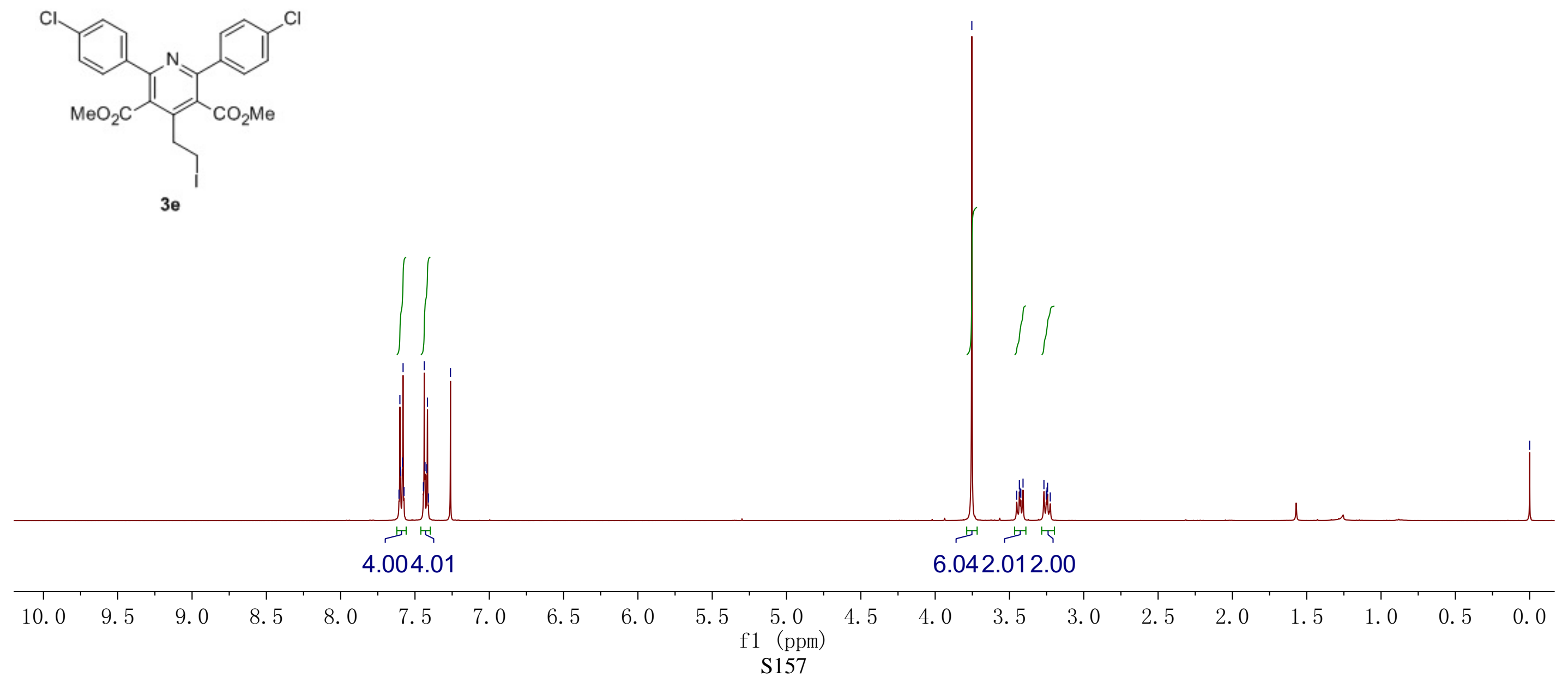
${ }^{13} \mathrm{C}$ NMR (100 MHz, $\left.\mathrm{CDCl}_{3}\right)$
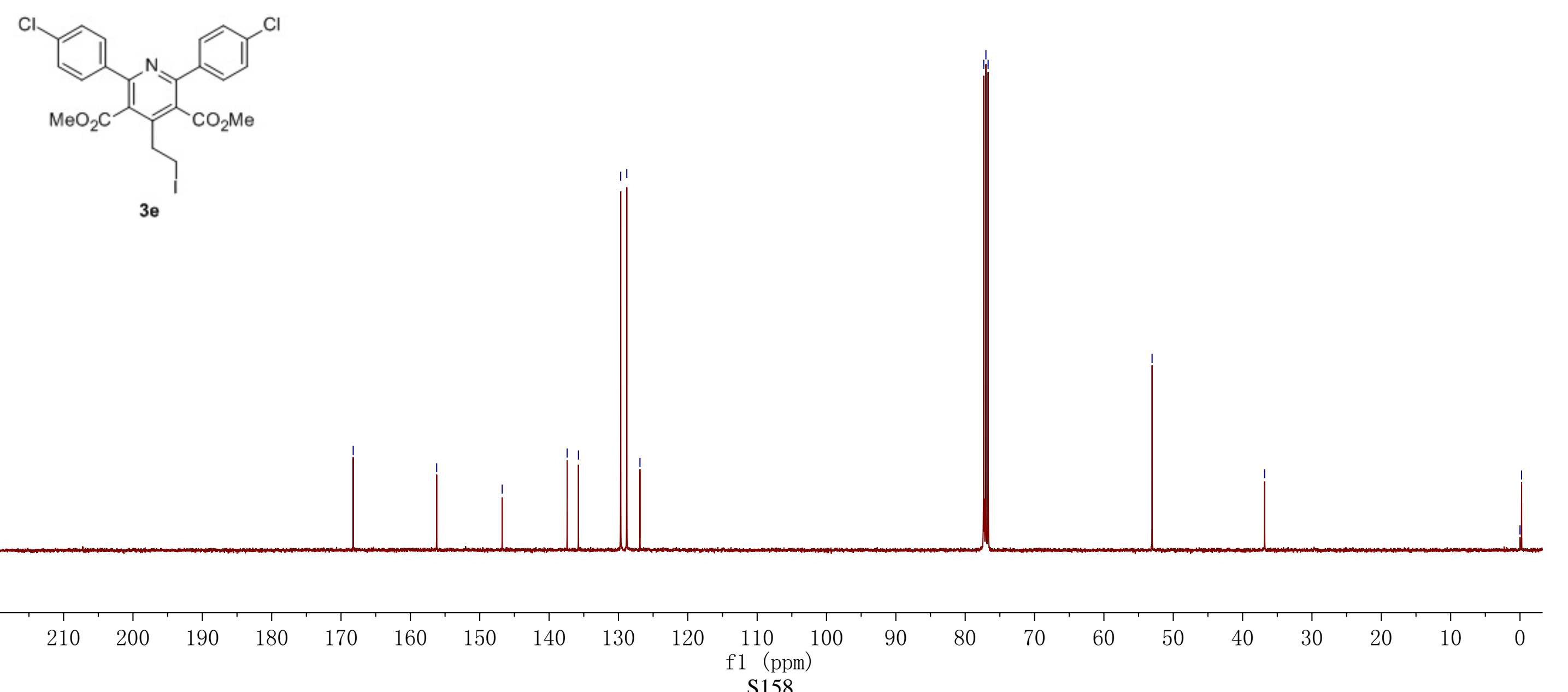


\section{${ }^{1} \mathrm{H}$ NMR (400 MHz, $\left.\mathrm{CDCl}_{3}\right)$}
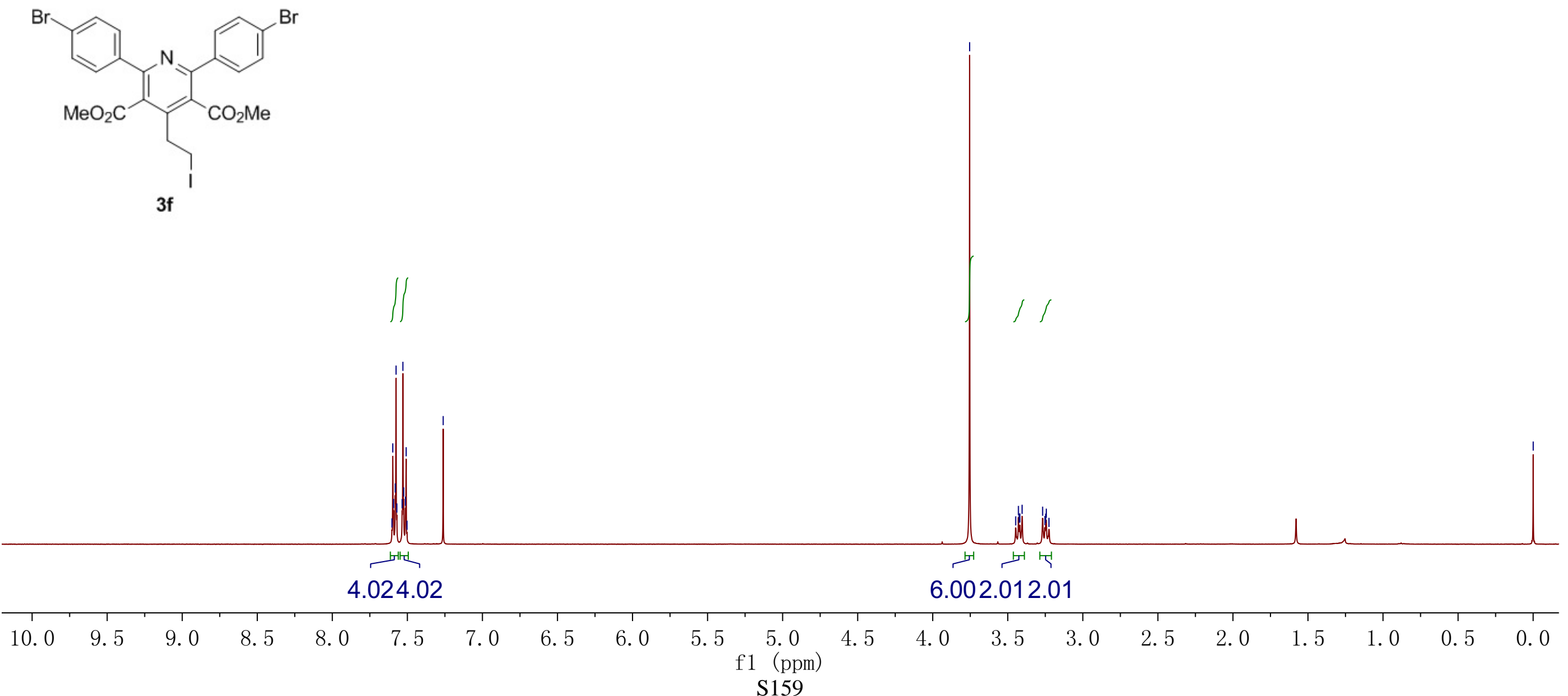
${ }^{13} \mathrm{C} \mathrm{NMR}\left(100 \mathrm{MHz}, \mathrm{CDCl}_{3}\right)$
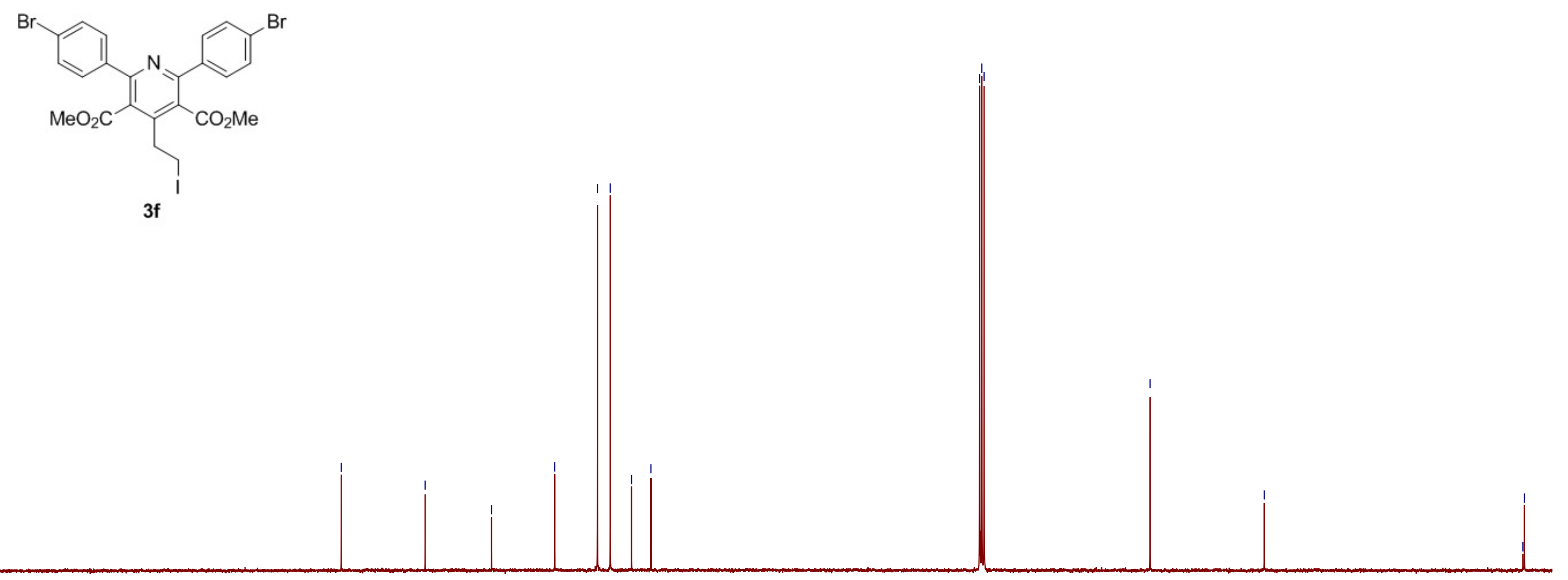
${ }^{1} \mathrm{H}$ NMR (400 MHz, $\mathrm{CDCl}_{3}$ )

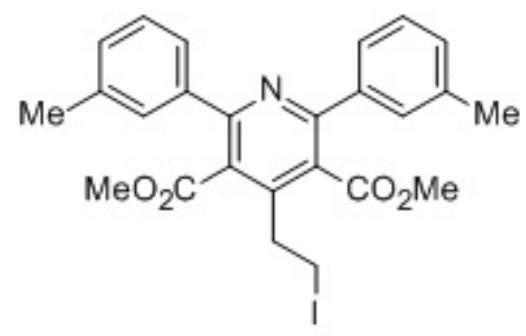

$3 \mathbf{i}$

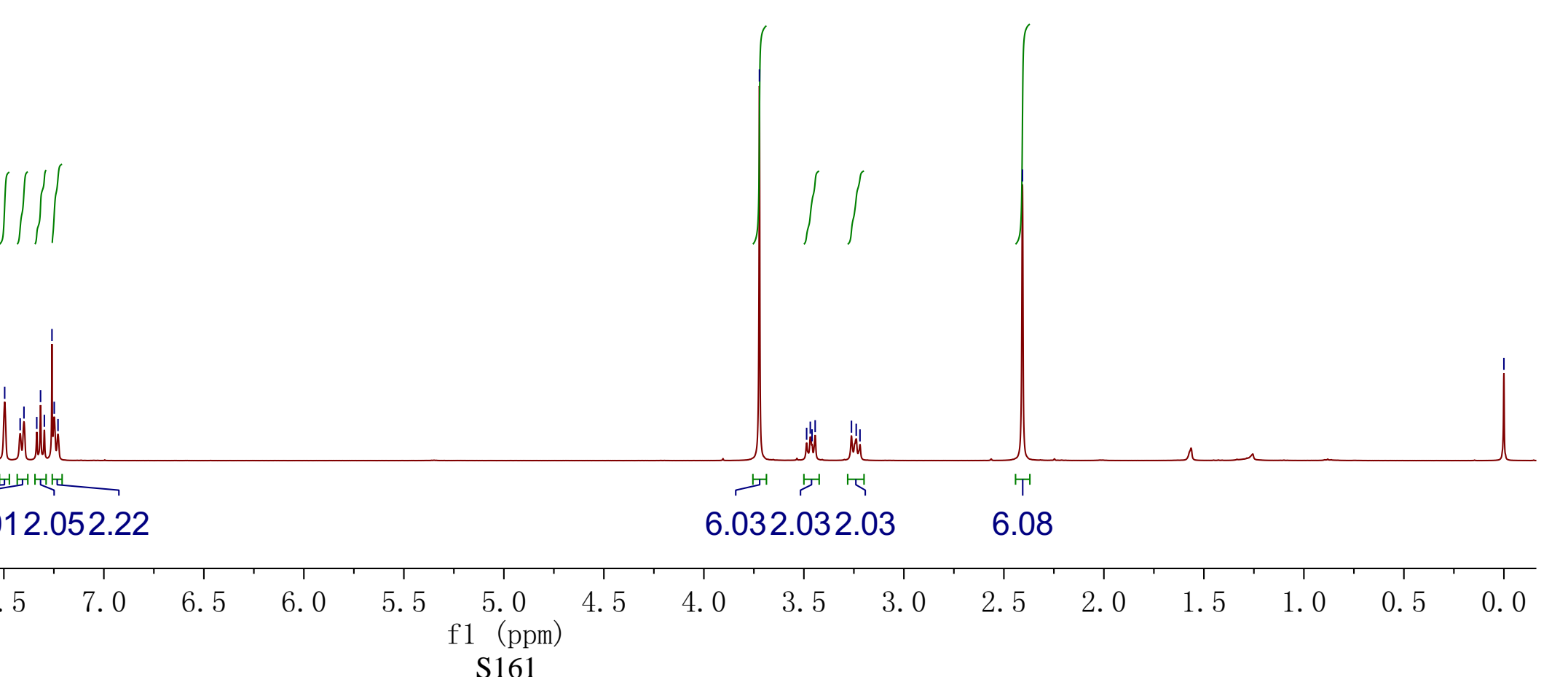




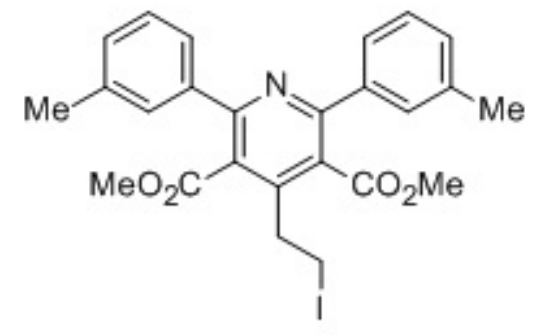

3i
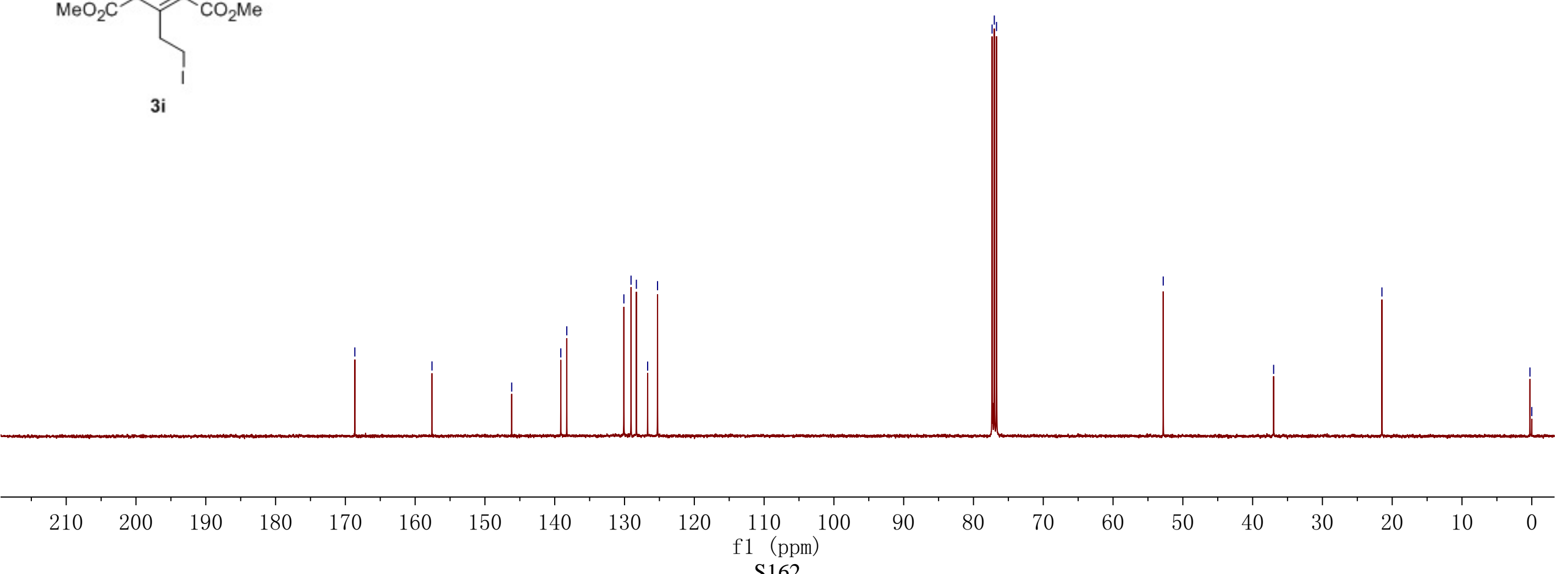


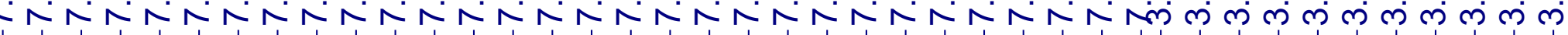

${ }^{1} \mathrm{H}$ NMR $\left(400 \mathrm{MHz}, \mathrm{CDCl}_{3}\right)$

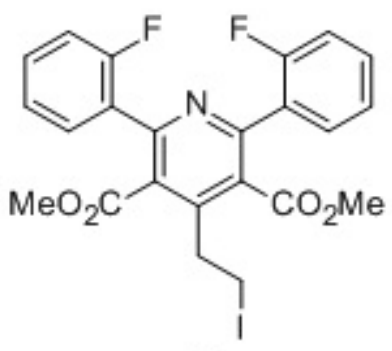

$3 \mathbf{j}$

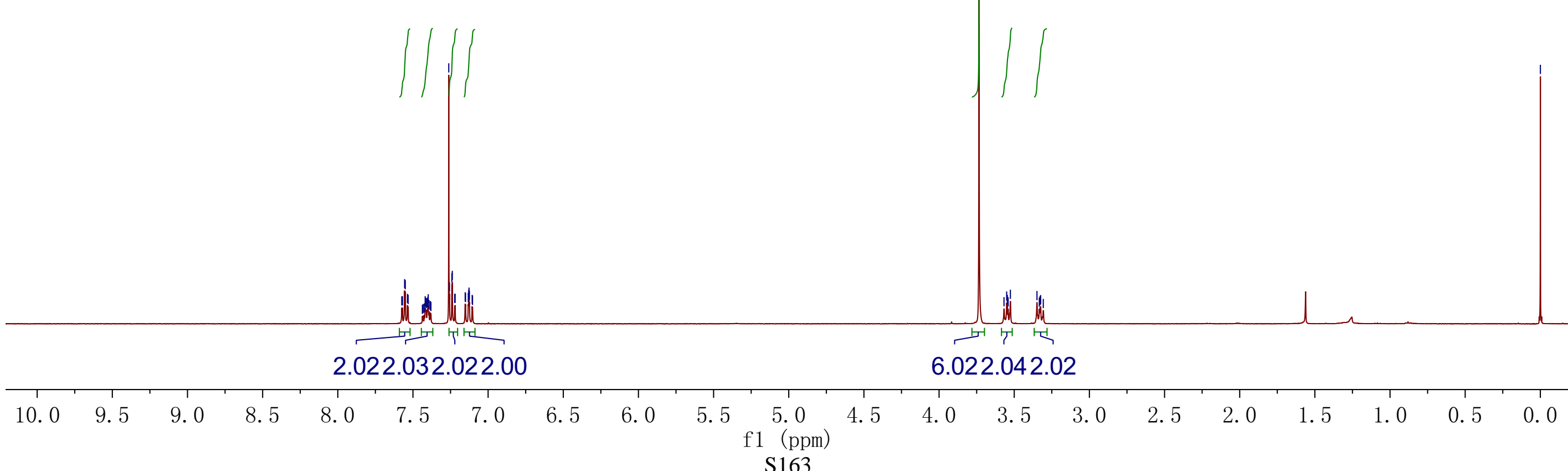


${ }^{13} \mathrm{C}$ NMR $\left(100 \mathrm{MHz}, \mathrm{CDCl}_{3}\right)$

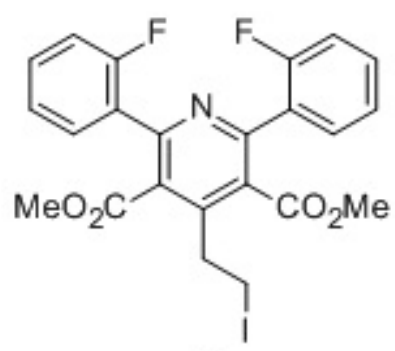

3j

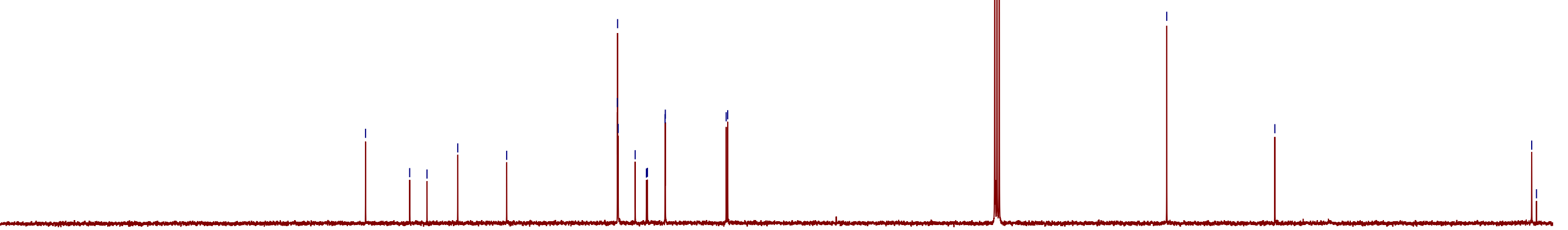


${ }^{19} \mathrm{~F}$ NMR (376 $\mathrm{MHz}, \mathrm{CDCl}_{3}$ )

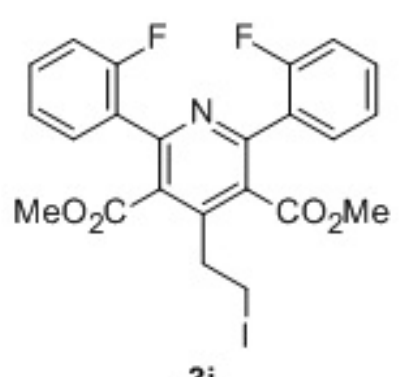

3j

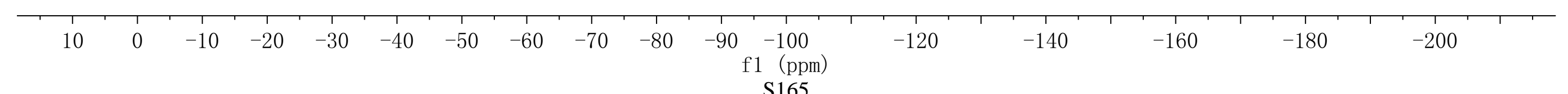




\section{${ }^{1} \mathrm{H} \mathrm{NMR}\left(400 \mathrm{MHz}, \mathrm{CDCl}_{3}\right)$}

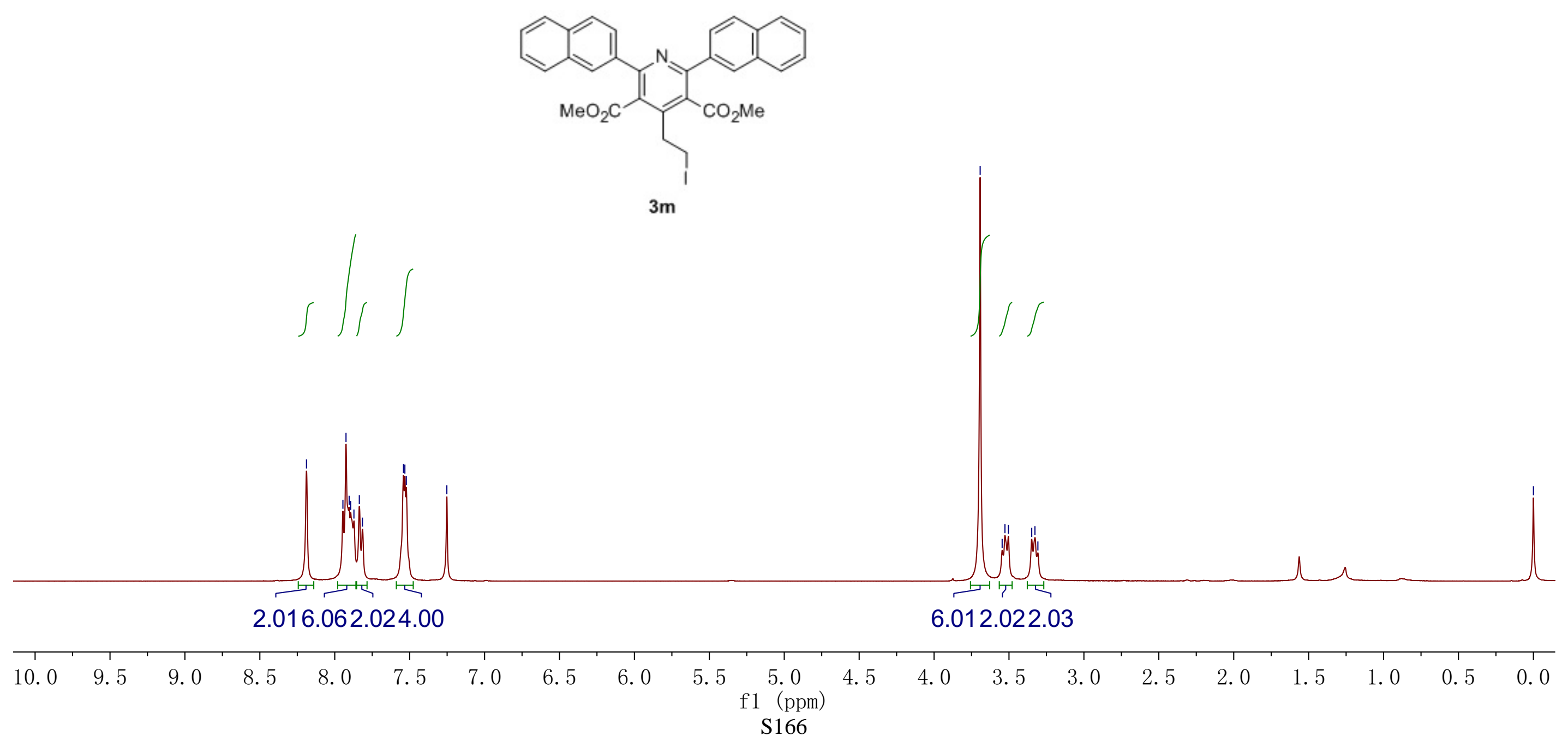


${ }^{13} \mathrm{C}$ NMR $\left(100 \mathrm{MHz}, \mathrm{CDCl}_{3}\right)$

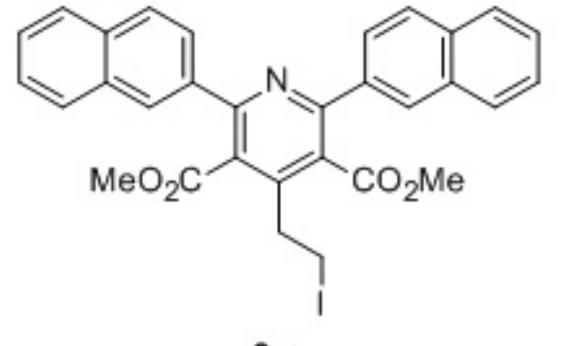

$3 m$

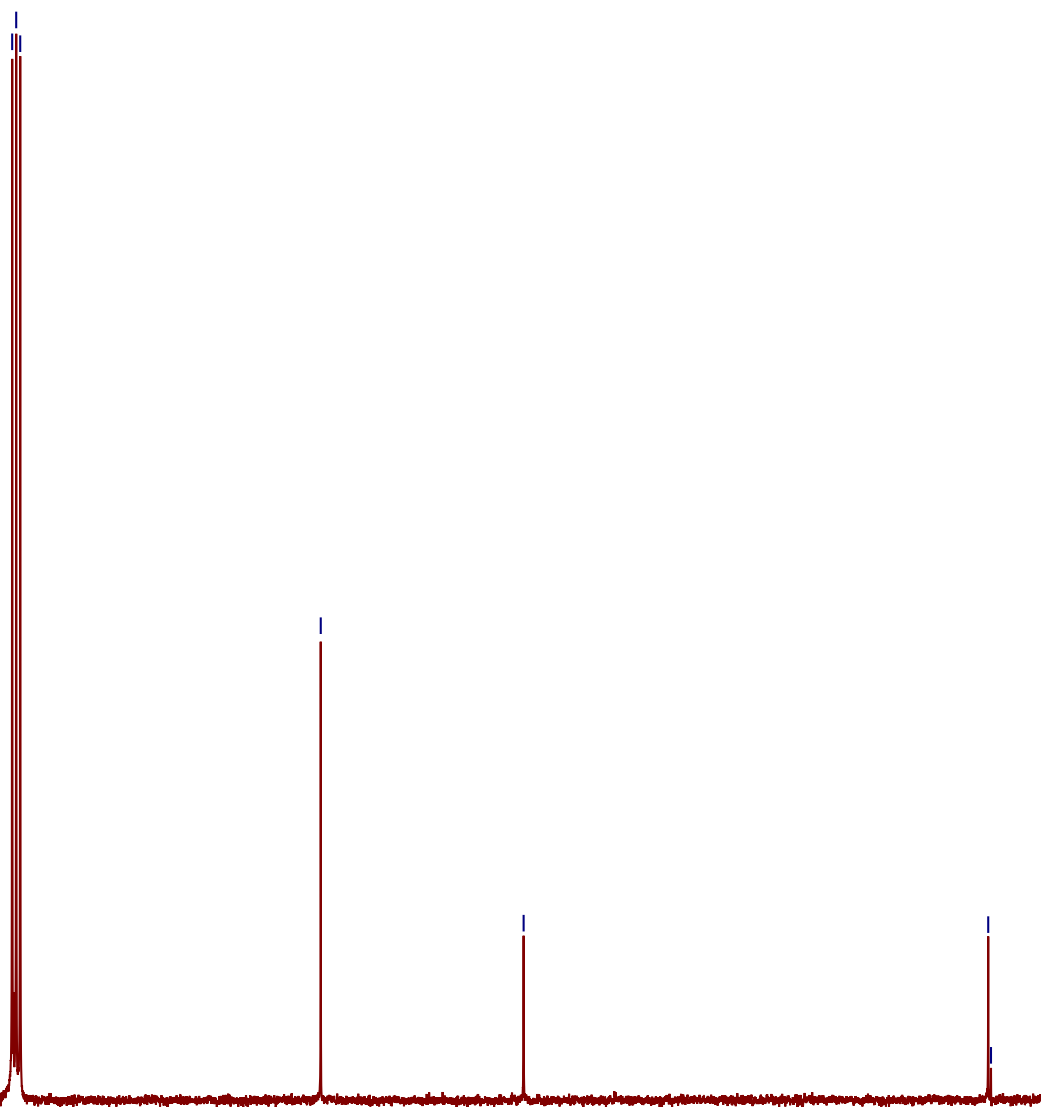

210

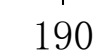




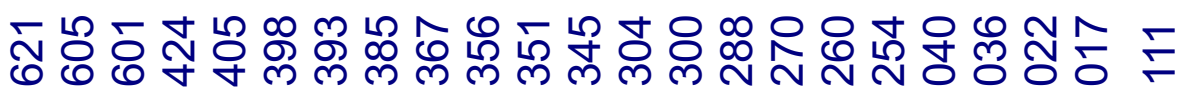

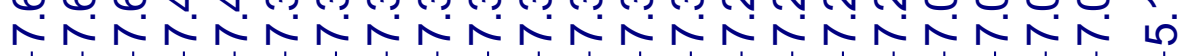

${ }^{1} \mathrm{H}$ NMR (400 MHz, $\mathrm{CDCl}_{3}$ )

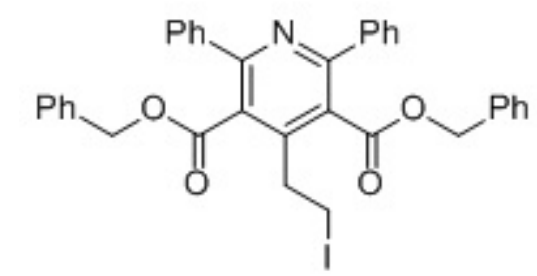

3o

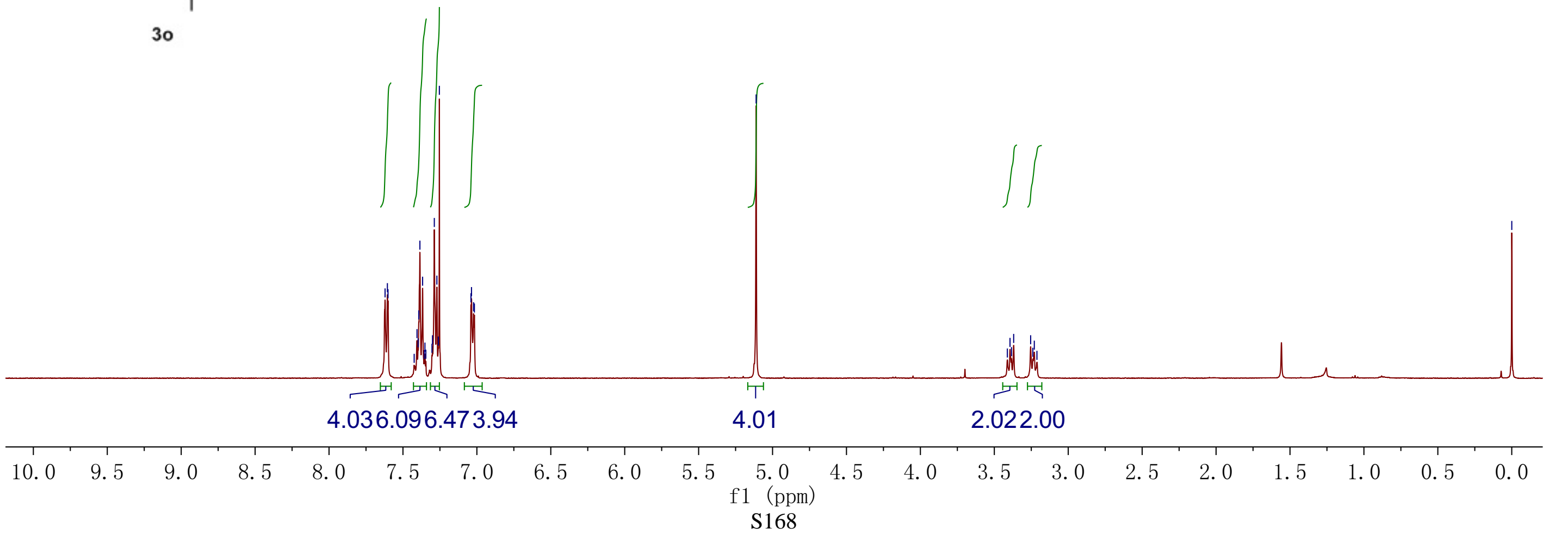


${ }^{13} \mathrm{C}$ NMR $\left(100 \mathrm{MHz}, \mathrm{CDCl}_{3}\right)$

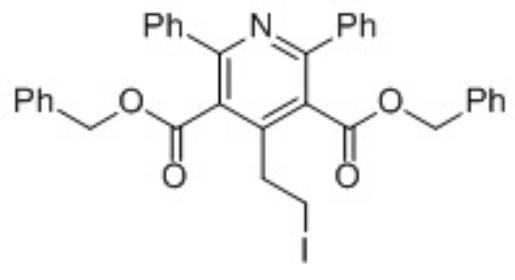

3o

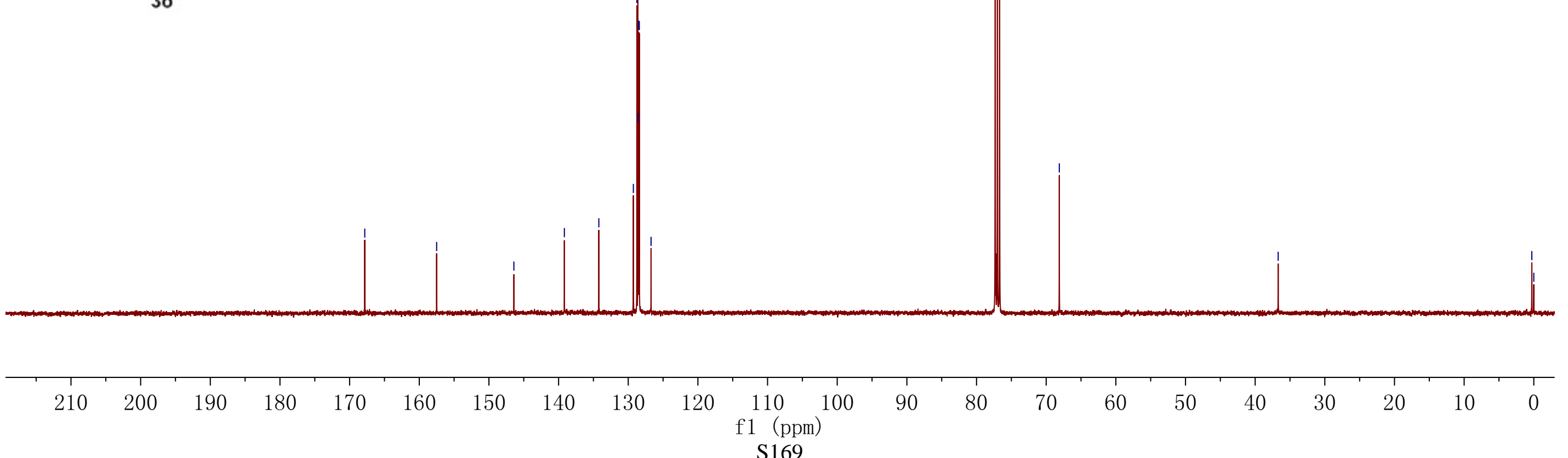


${ }^{1} \mathrm{H}$ NMR (400 MHz, $\mathrm{CDCl}_{3}$ )

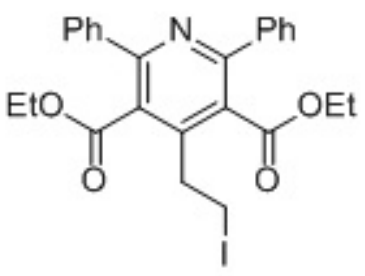

$3 \mathrm{~s}$

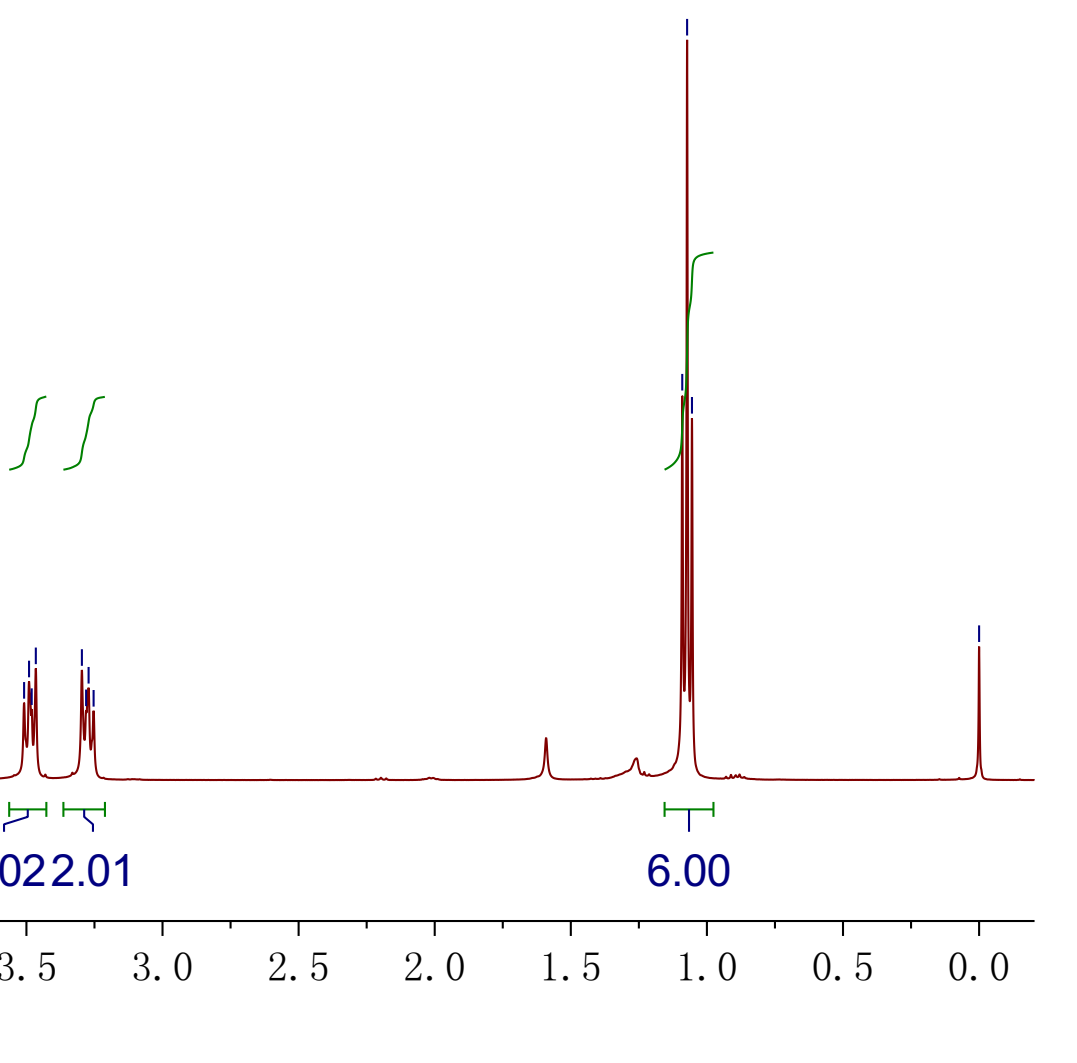


${ }^{13} \mathrm{C}$ NMR (100 MHz, $\left.\mathrm{CDCl}_{3}\right)$

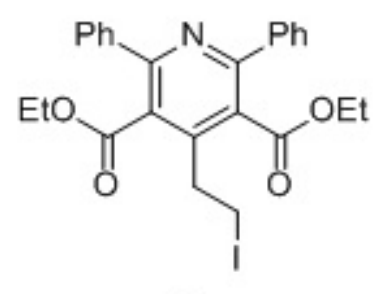

3s

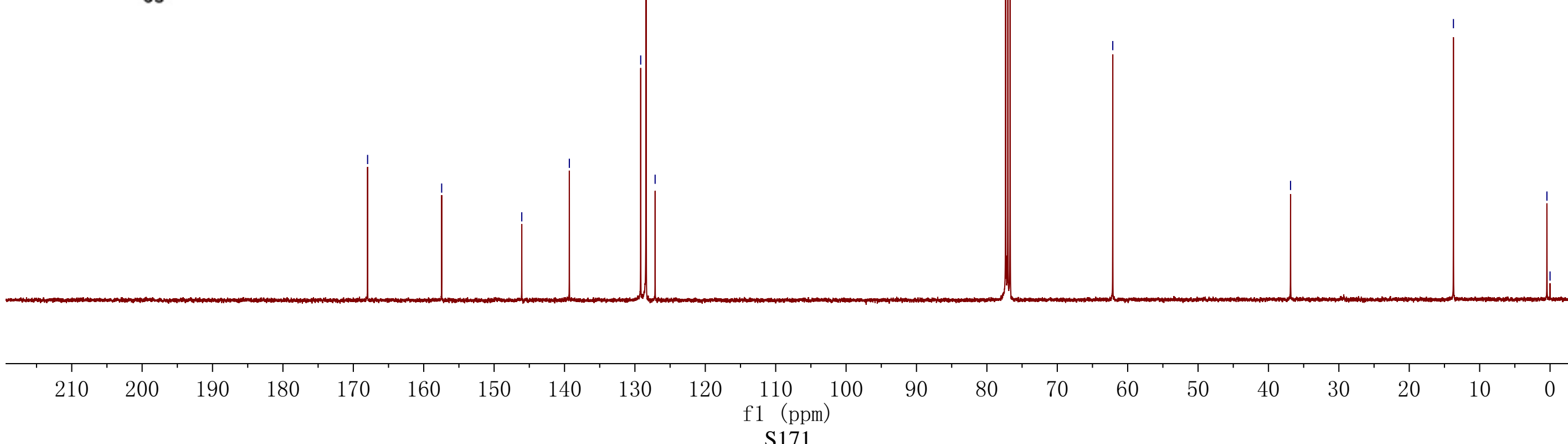




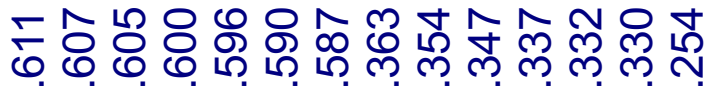

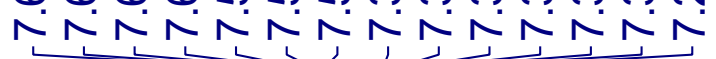

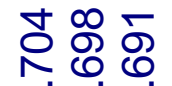

身

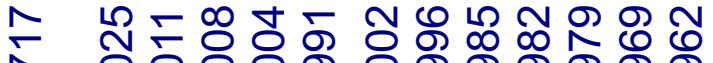

त.

m m m m m

${ }^{1} \mathrm{H}$ NMR (400 MHz, $\mathrm{CDCl}_{3}$ )
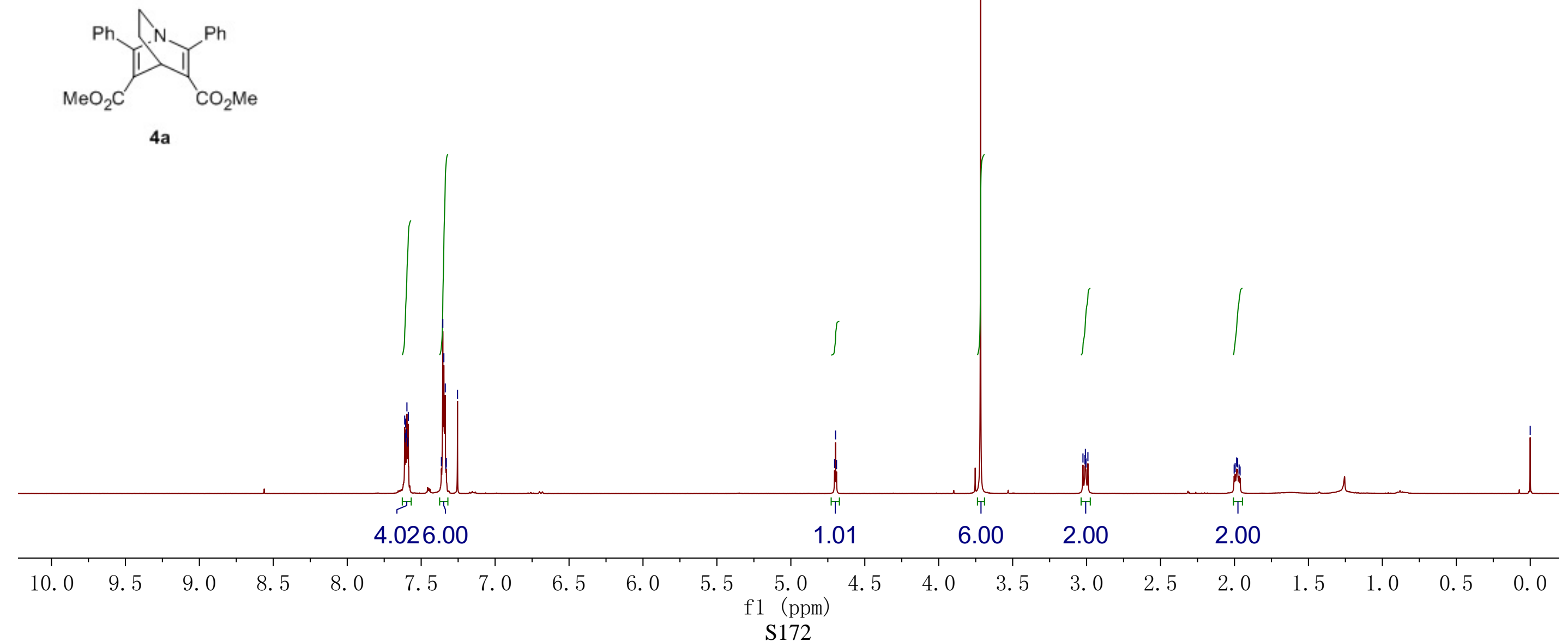
บ N

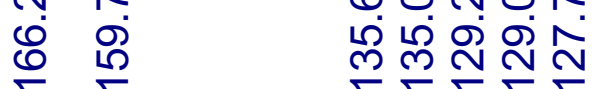

I
$M O N$

NN

${ }^{13} \mathrm{C} \mathrm{NMR}\left(100 \mathrm{MHz}, \mathrm{CDCl}_{3}\right)$

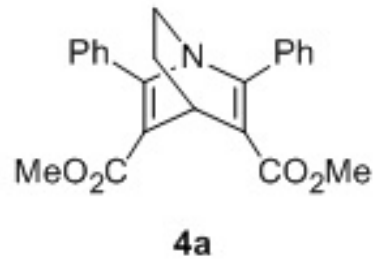

210

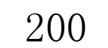

190 
${ }^{1} \mathrm{H} \mathrm{NMR}\left(400 \mathrm{MHz}, \mathrm{CDCl}_{3}\right)$

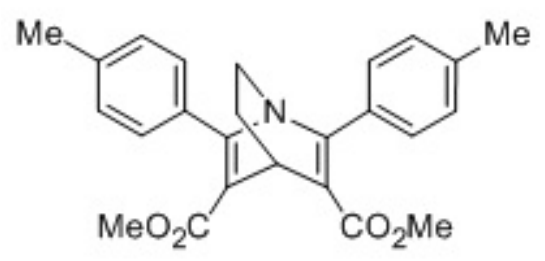

4b

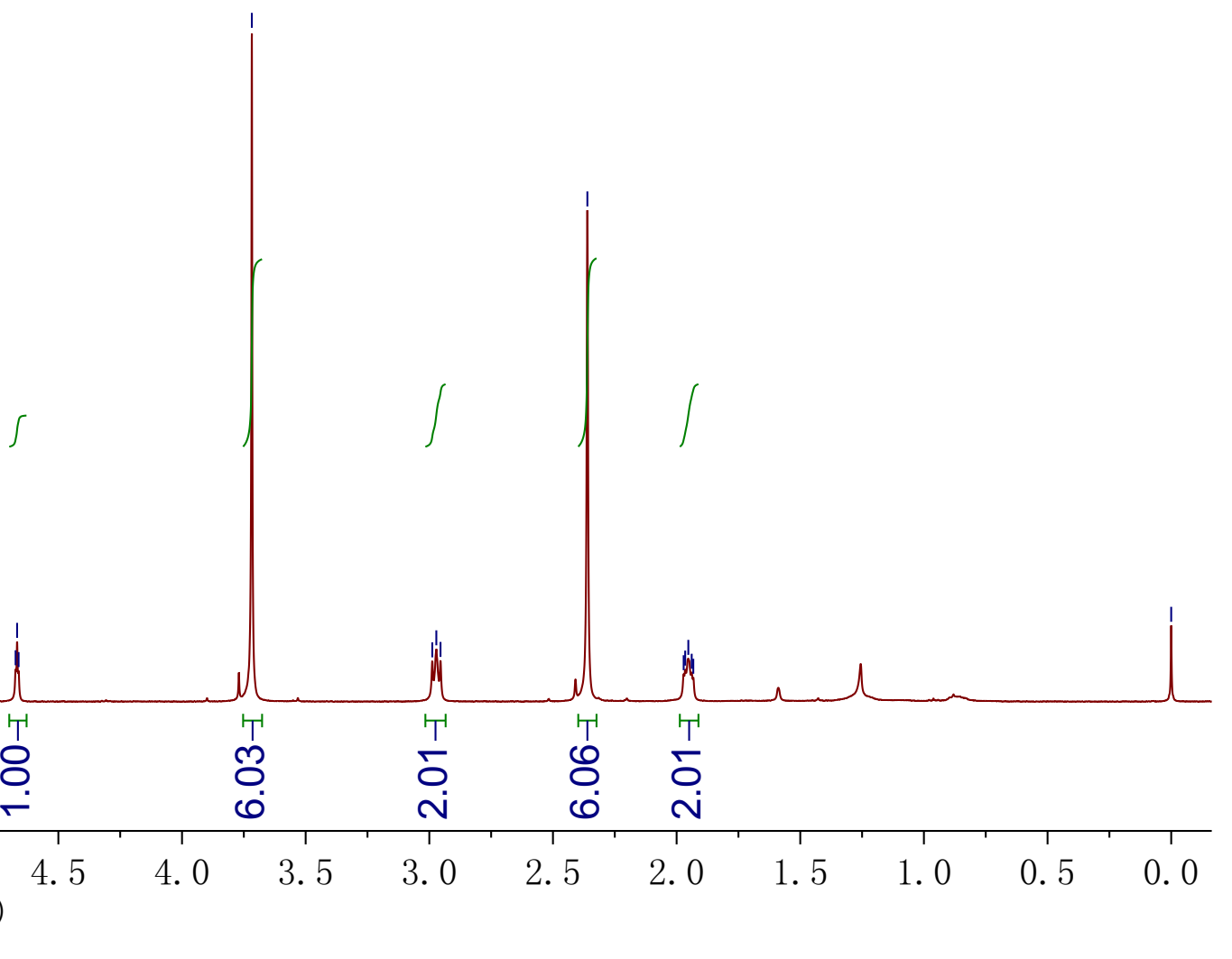


ペ゚

TरN

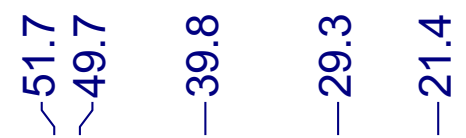

${ }^{13} \mathrm{C} \mathrm{NMR}\left(100 \mathrm{MHz}, \mathrm{CDCl}_{3}\right)$

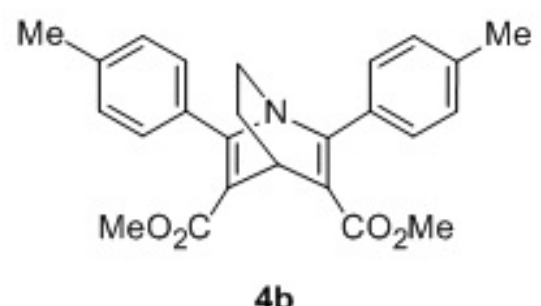

$4 b$

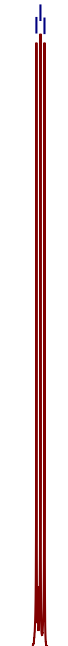

200

190

180

170

160

150

140

130

120

110

1 (ppm) 
${ }^{1} \mathrm{H}$ NMR (400 MHz, $\mathrm{CDCl}_{3}$ )

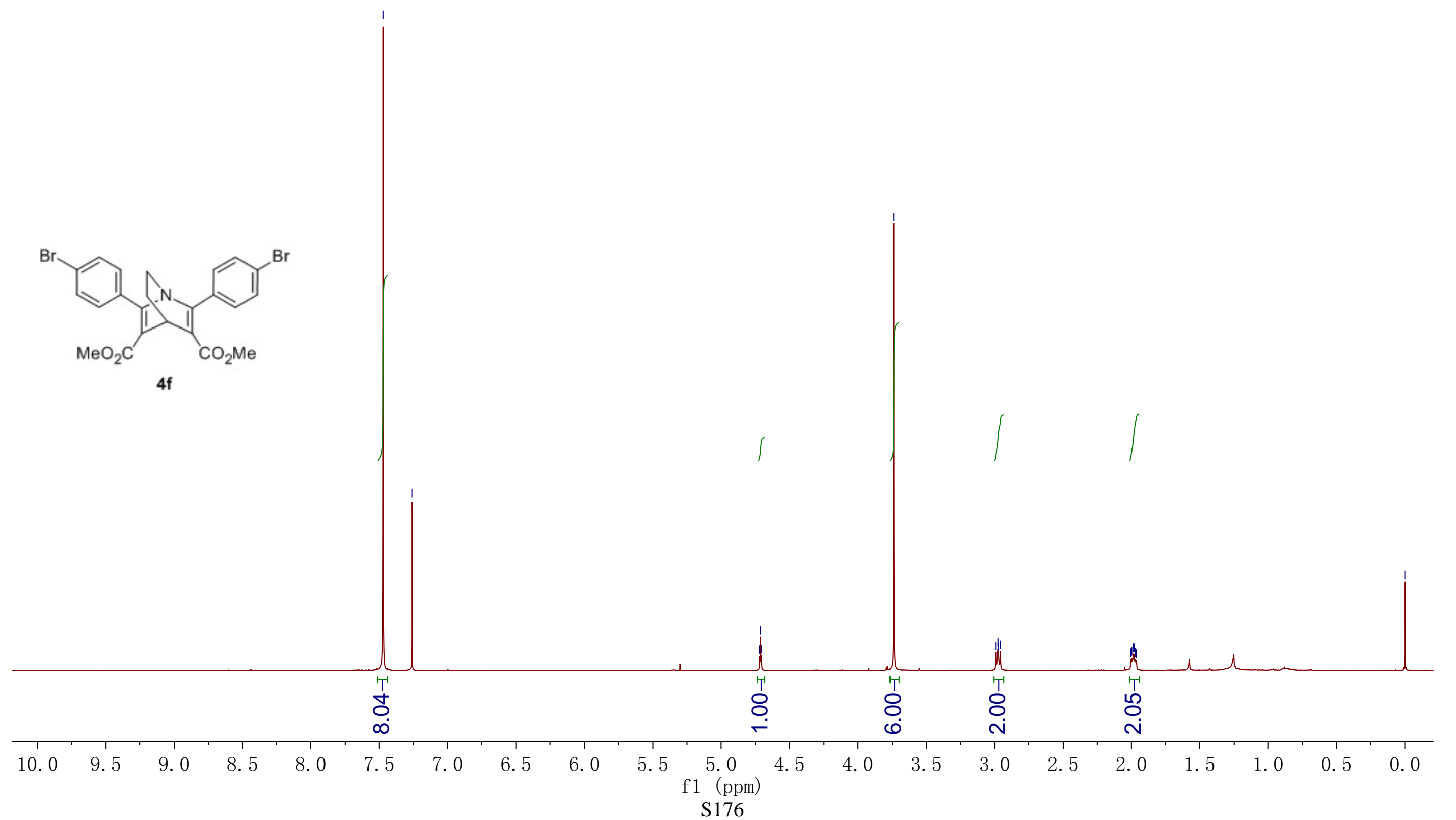




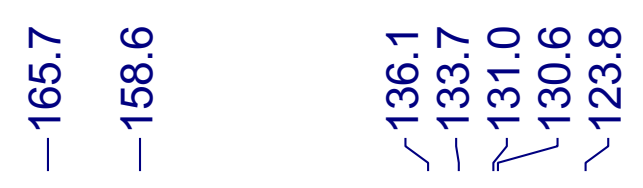

ペ゚゚

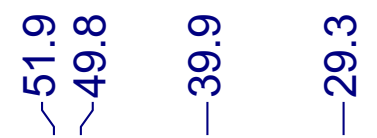

\section{${ }^{13} \mathrm{C}$ NMR (100 MHz, $\left.\mathrm{CDCl}_{3}\right)$}
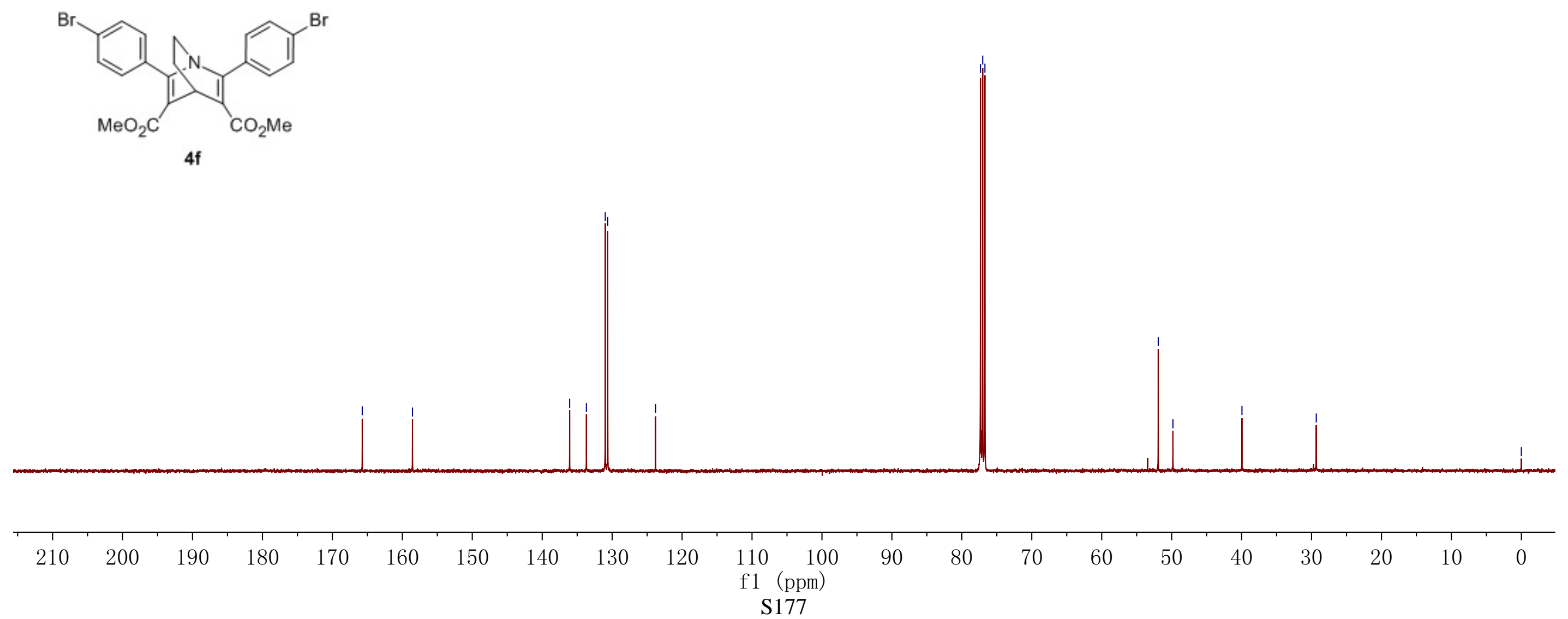
${ }^{1} \mathrm{H} \mathrm{NMR}\left(400 \mathrm{MHz}, \mathrm{CDCl}_{3}\right)$

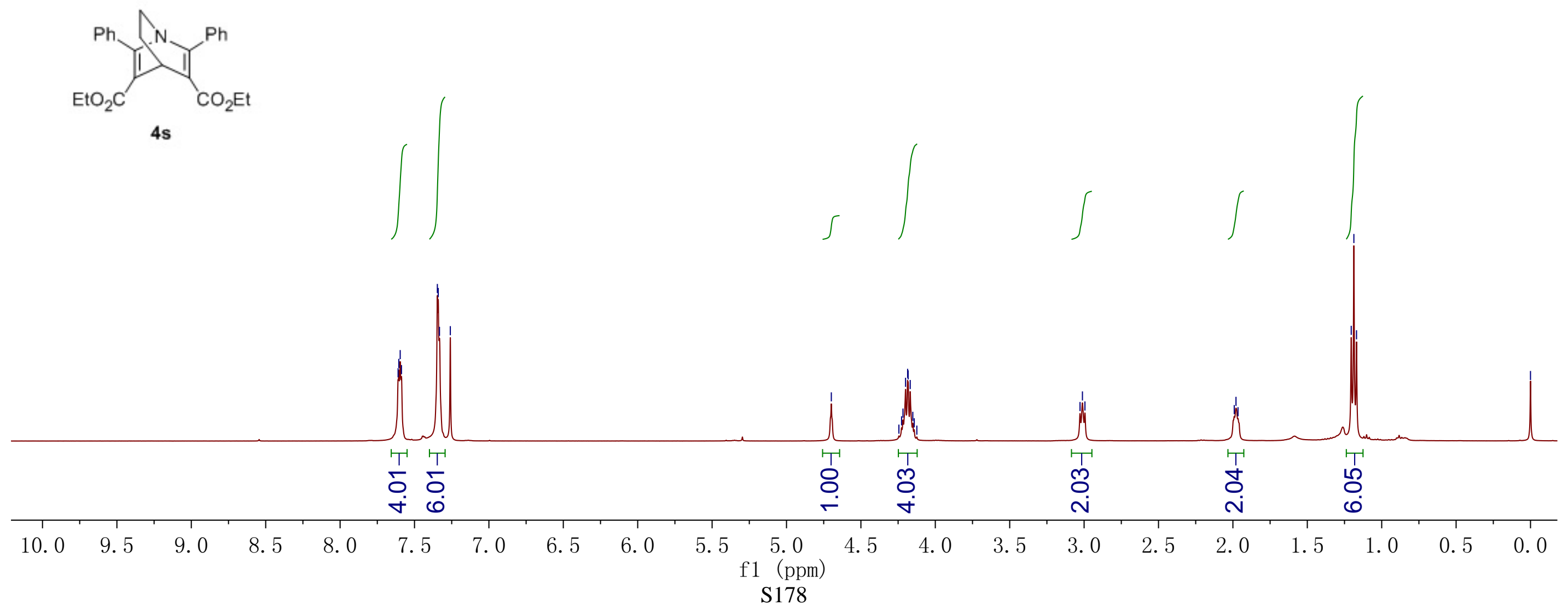


${ }^{13} \mathrm{C} \mathrm{NMR}\left(100 \mathrm{MHz}, \mathrm{CDCl}_{3}\right)$
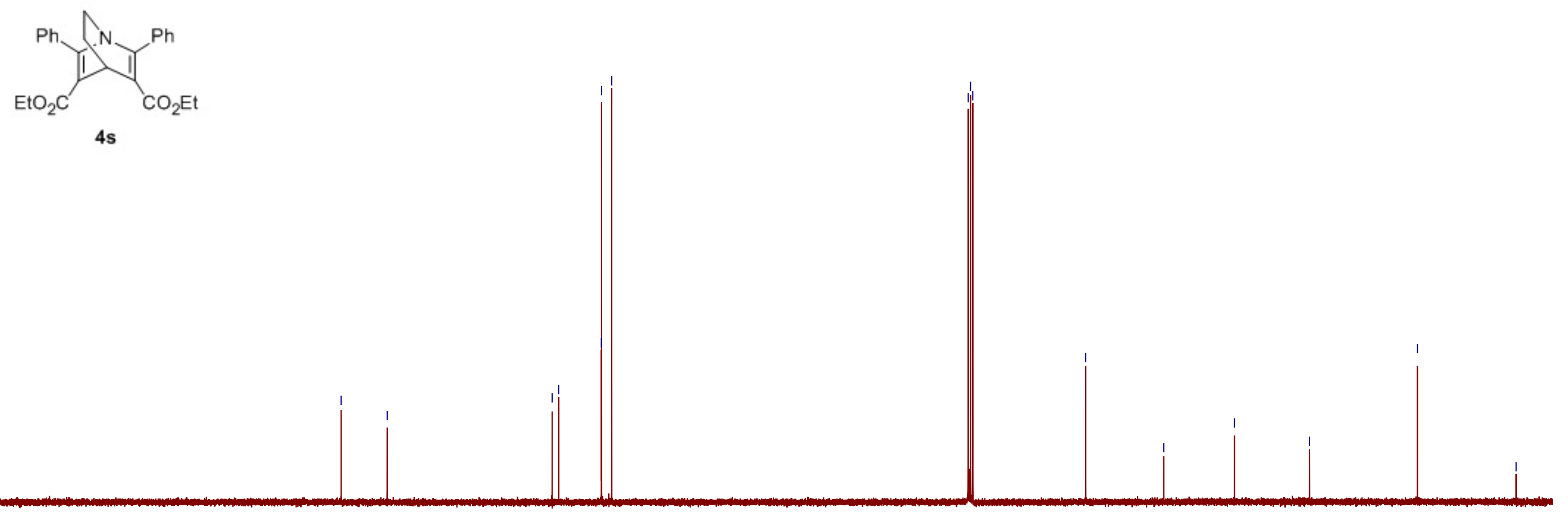

$210 \quad 200$

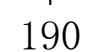


${ }^{1} \mathrm{H}$ NMR (400 MHz, $\mathrm{CDCl}_{3}$ )
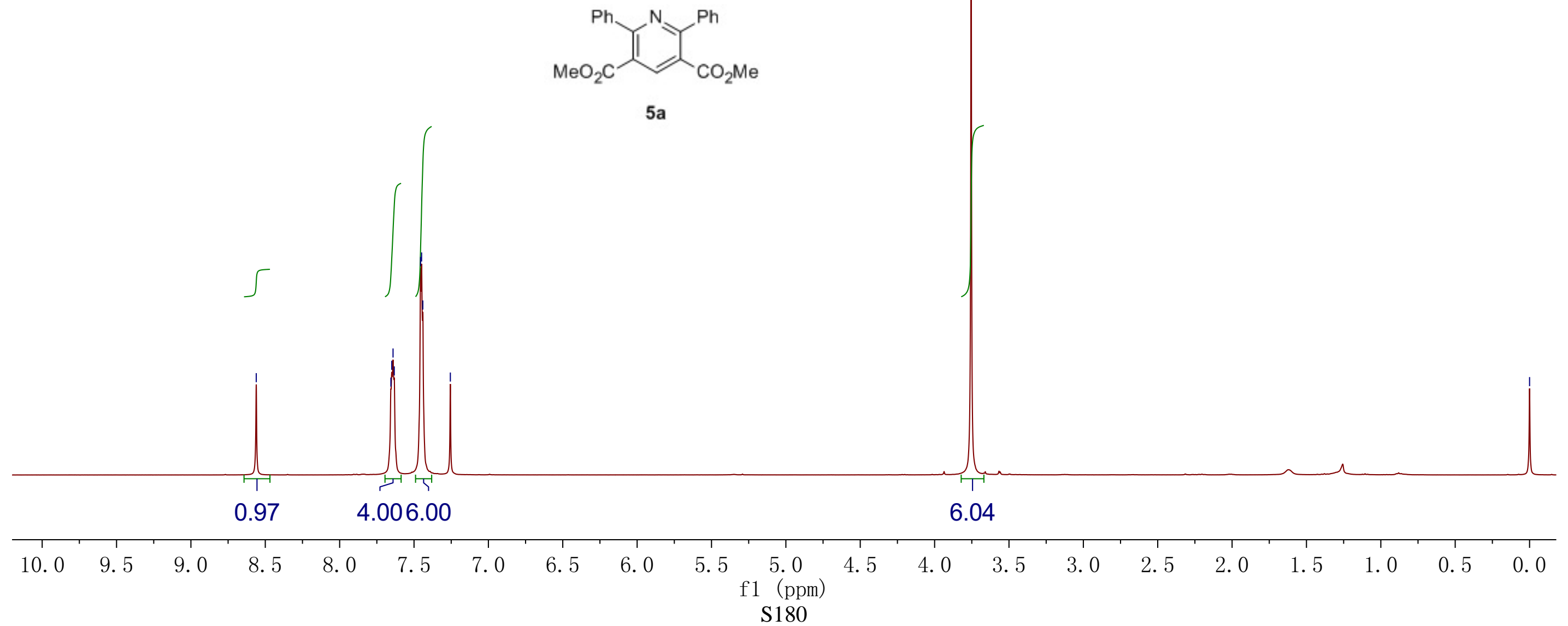


\section{${ }^{13} \mathrm{C}$ NMR (100 MHz, $\left.\mathrm{CDCl}_{3}\right)$}
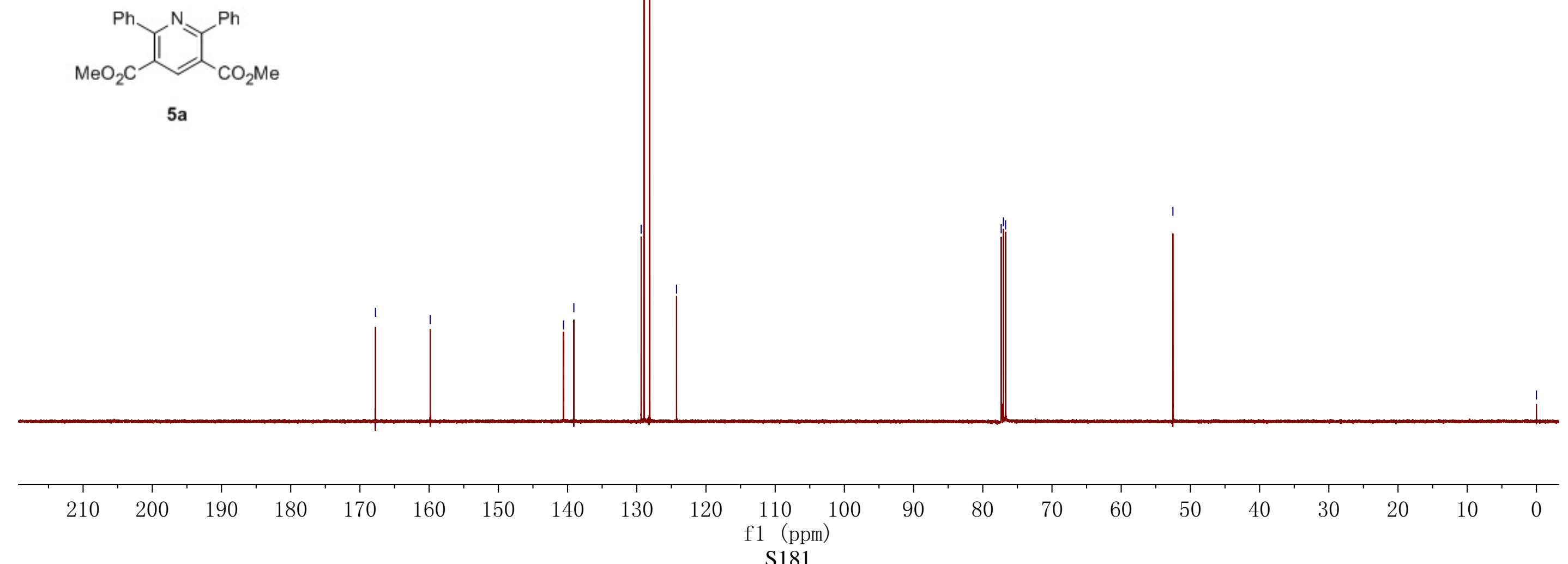


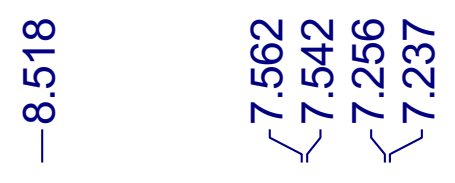

${ }^{1} \mathrm{H} \mathrm{NMR}\left(400 \mathrm{MHz}, \mathrm{CDCl}_{3}\right)$
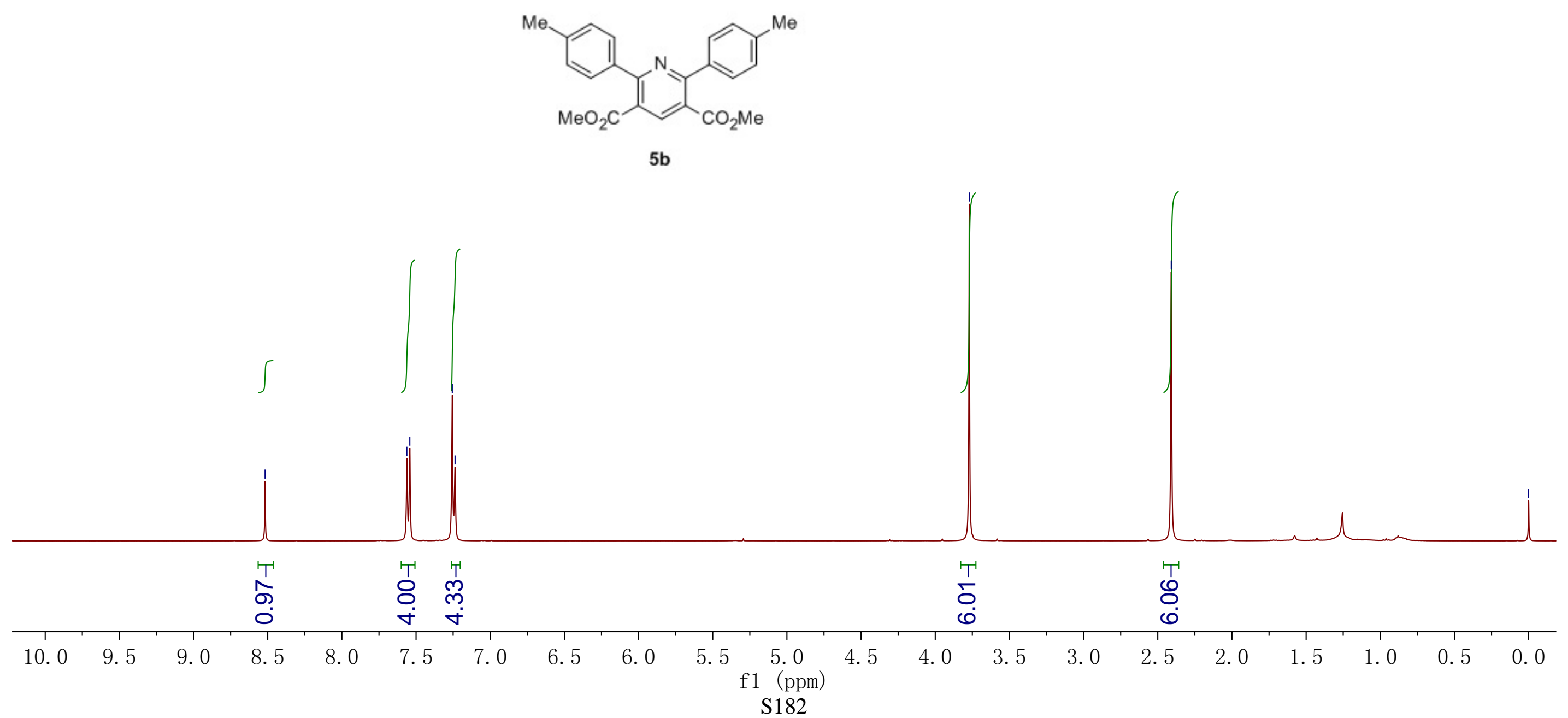

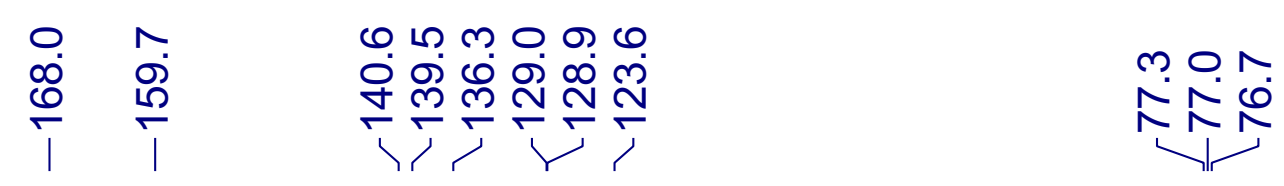

กิ่

${ }^{13} \mathrm{C}$ NMR (100 MHz, $\left.\mathrm{CDCl}_{3}\right)$

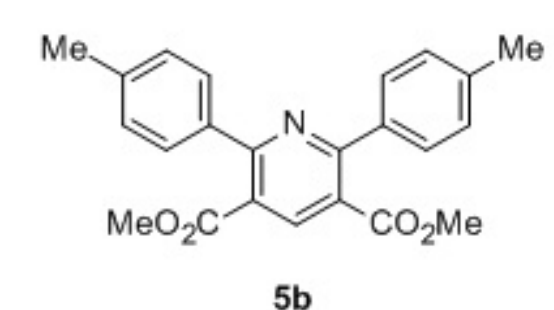

$5 b$
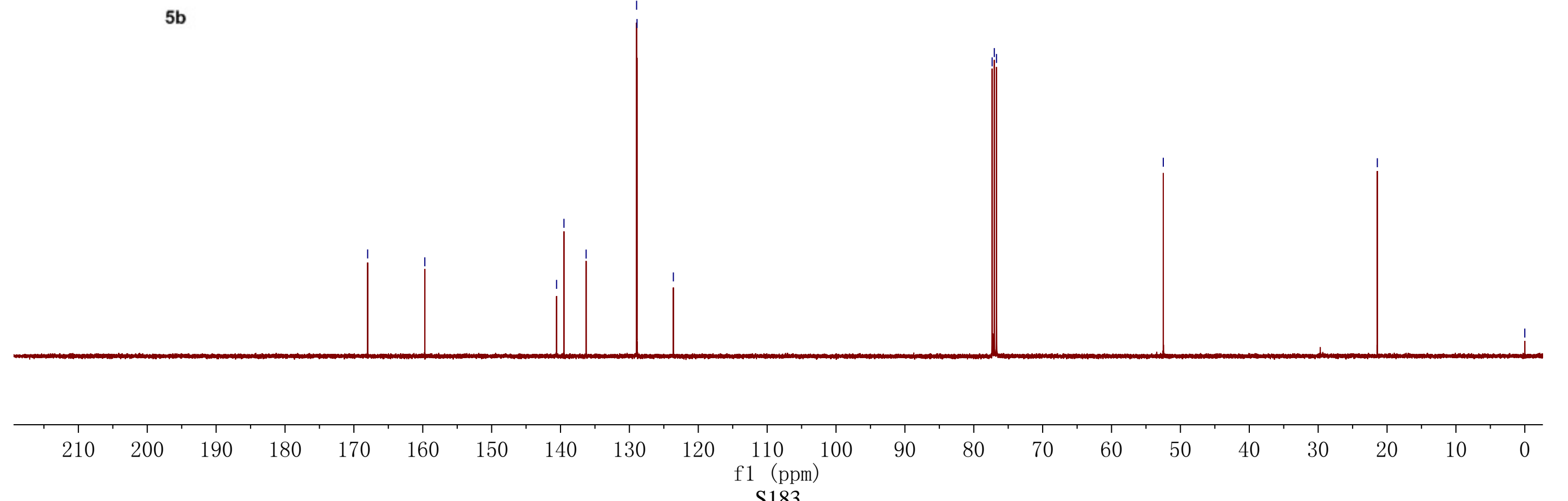
${ }^{1} \mathrm{H}$ NMR (400 MHz, $\mathrm{CDCl}_{3}$ )
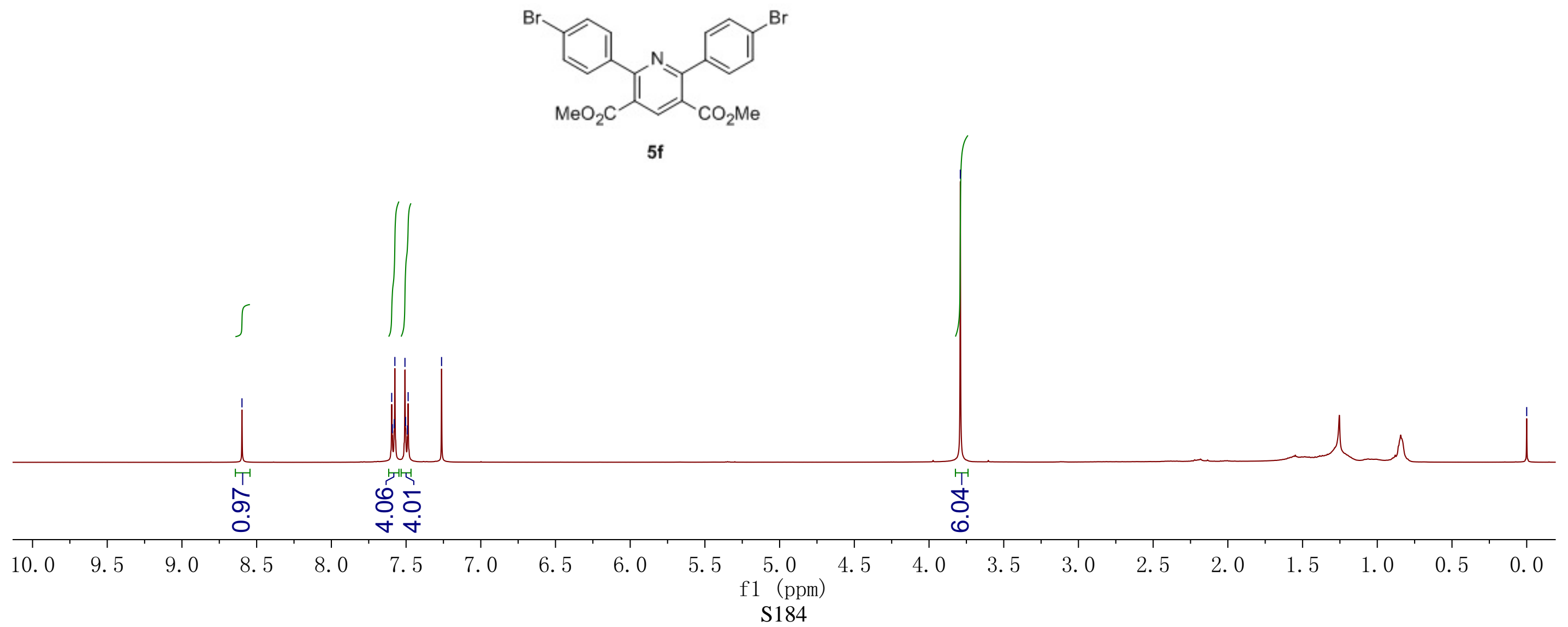
ڤ̊

솟

กิ่

웅

${ }^{13} \mathrm{C}$ NMR (100 MHz, $\left.\mathrm{CDCl}_{3}\right)$

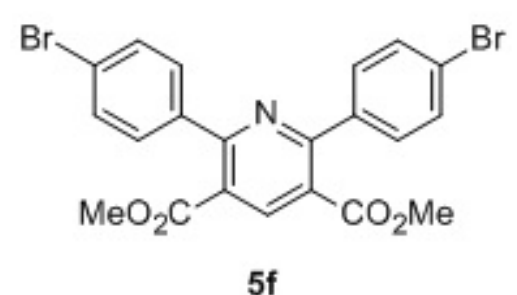

$5 f$

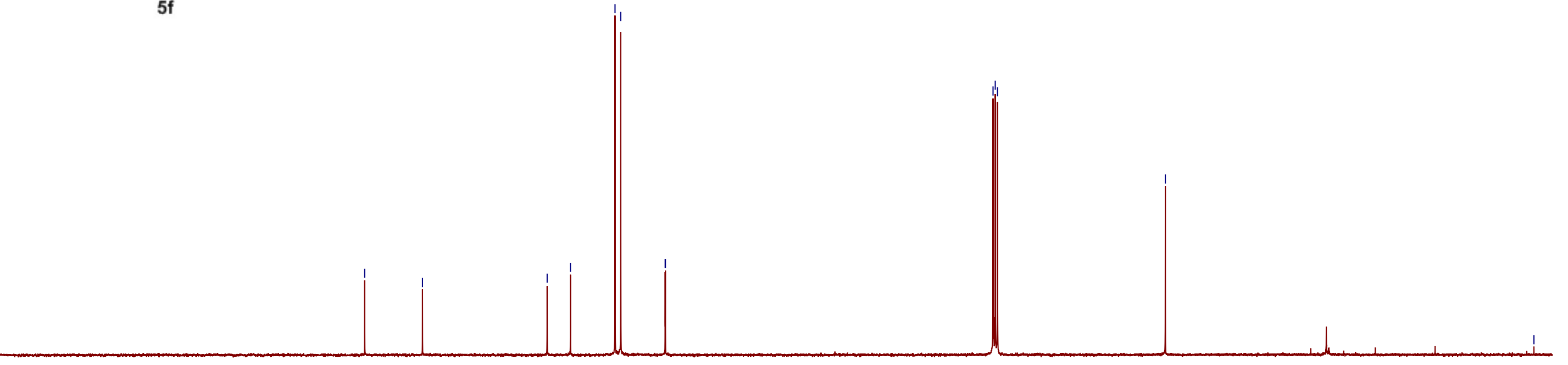


${ }^{1} \mathrm{H}$ NMR (400 MHz, $\mathrm{CDCl}_{3}$ )
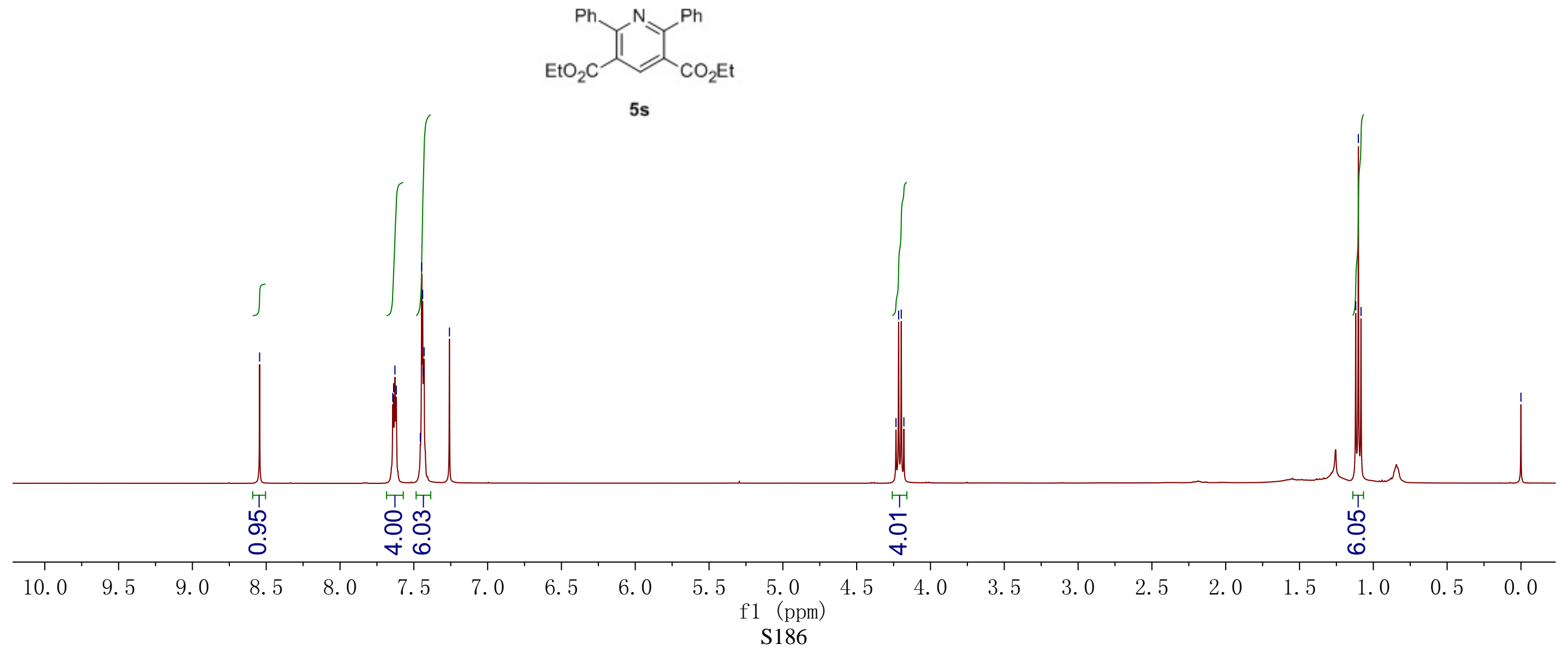


\section{${ }^{13} \mathrm{C}$ NMR (100 MHz, $\left.\mathrm{CDCl}_{3}\right)$}

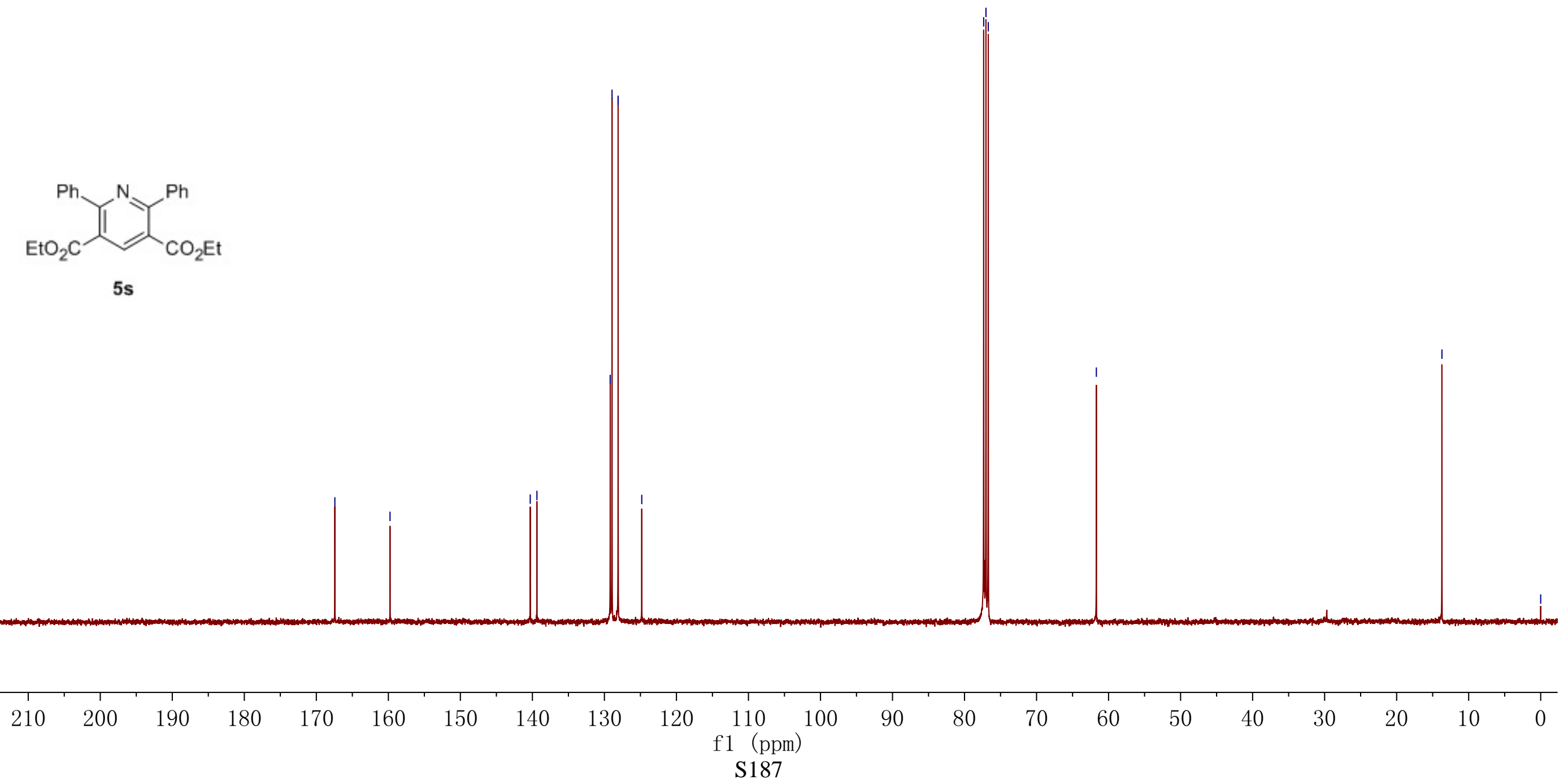

